\title{
Chlamydia trachomatis testing policy and control : the neglected role of the anorectal site
}

Citation for published version (APA):

van Liere, G. A. F. S. (2015). Chlamydia trachomatis testing policy and control : the neglected role of the anorectal site. [Doctoral Thesis, Maastricht University]. Maastricht University. https://doi.org/10.26481/dis.20151214gl

Document status and date:

Published: 01/01/2015

DOI:

10.26481/dis.20151214gl

Document Version:

Publisher's PDF, also known as Version of record

\section{Please check the document version of this publication:}

- A submitted manuscript is the version of the article upon submission and before peer-review. There can be important differences between the submitted version and the official published version of record.

People interested in the research are advised to contact the author for the final version of the publication, or visit the DOI to the publisher's website.

- The final author version and the galley proof are versions of the publication after peer review.

- The final published version features the final layout of the paper including the volume, issue and page numbers.

Link to publication

\footnotetext{
General rights rights.

- You may freely distribute the URL identifying the publication in the public portal. please follow below link for the End User Agreement:

www.umlib.nl/taverne-license

Take down policy

If you believe that this document breaches copyright please contact us at:

repository@maastrichtuniversity.nl

providing details and we will investigate your claim.
}

Copyright and moral rights for the publications made accessible in the public portal are retained by the authors and/or other copyright owners and it is a condition of accessing publications that users recognise and abide by the legal requirements associated with these

- Users may download and print one copy of any publication from the public portal for the purpose of private study or research.

- You may not further distribute the material or use it for any profit-making activity or commercial gain

If the publication is distributed under the terms of Article $25 \mathrm{fa}$ of the Dutch Copyright Act, indicated by the "Taverne" license above, 


\section{Chlamydia trachomatis testing policy and control: the neglected role of the anorectal site}

Geneviève van Liere,

Academische Werkplaats Publieke Gezondheid Limburg 
(C) Copywright: Geneviève van Liere, Maastricht 2015

Design \& Lay-out: Margret Reijnders | Creatieve Communicatie

Photo (cover): Remco Graham and Richard Holmes, 2014

ISBN: 978-90-823808-2-8

No part of this thesis may be reproduced, stored, or transmitted in any form or by any means, electronic or mechanical, including photocopy, recording or any information storage or retrieval system, without prior permission of the copyright owner.

This thesis was prepared at the Department of Sexual Health (GGD Zuid Limburg), Infectious Diseases and Environmental Health at the Public Health Service South Limburg, the School of Public Health and Primary Care (CAPHRI) and the Department of Medical Microbiology at the Maastricht University Medical Centre.

The printing of this thesis was financially supported by: GGD Zuid Limburg.
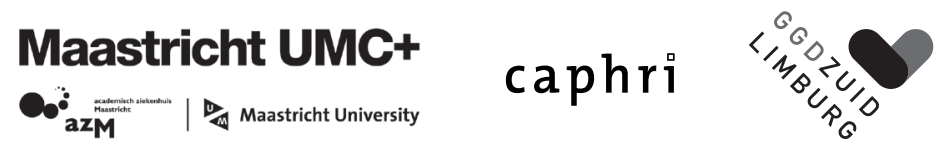


\title{
Chlamydia trachomatis testing policy and control: the neglected role of the anorectal site
}

\author{
Proefschrift
}

ter verkrijging van de graad van doctor aan de Universiteit Maastricht, op gezag van de Rector Magnificus, Prof dr. L.L.G. Soete volgens het besluit van het College van Decanen,

in het openbaar te verdedigen op maandag 14 december 2015 om 12.00 uur door

Geneviève Astrid Francisca Sophia van Liere

Geboren op 15 april 1987 te Heerlen. 


\section{Promotor:}

Prof. dr. C.J.P.A. Hoebe

\section{Co-promotor:}

Dr. N.H.T.M. Dukers-Muijrers

\section{Beoordelingscommissie:}

Prof. dr. P.H.M. Savelkoul (voorzitter)

Prof. dr. S.A. Morré

Prof. dr. M. Prins, Universiteit van Amsterdam

Prof. dr. J.H. Richardus, Erasmus MC Rotterdam

Prof. dr. R.A.C. Ruiter 
Dit proefschrift is tot stand gekomen vanuit de Academische Werkplaats Publieke Gezondheid Limburg, een samenwerkingsverband tussen de GGD Zuid Limburg, de faculteit Health, Medicine and Life Sciences van de Universiteit Maastricht en de 18 Zuid-Limburgse gemeenten. Het gepresenteerde onderzoek is uitgevoerd bij de School for Public Health and Primary Care: CAPHRI. CAPHRI participeert in de School of Primary Care Research (CaRe). 



\section{List of abbreviations}

$\begin{array}{ll}\text { AIDS } & \text { Acquired Immune Deficiency Syndrome } \\ \text { CAPHRI } & \text { School for Public Health and Primary Care } \\ \text { CDC } & \text { Centers for Disease Control and Prevention } \\ \text { CI } & \text { Confidence interval } \\ \text { CSW } & \text { Commercial sex worker } \\ \text { CT } & \text { Chlamydia trachomatis } \\ \text { HIV } & \text { Human immunodeficiency virus } \\ \text { LGV } & \text { Lymphogranuloma venereum } \\ \text { MSM } & \text { Men who have sex with men } \\ \text { NA } & \text { Not assessed } \\ \text { NAAT } & \text { Nucleic acid amplification test } \\ \text { NG } & \text { Neisseria gonorrhoeae } \\ \text { Omp A } & \text { Outer membrane protein A } \\ \text { OR } & \text { Odds ratio } \\ \text { PCR } & \text { Polymerase Chain Reaction } \\ \text { PID } & \text { Pelvic Inflammatory Disease } \\ \text { SDA } & \text { Strand displacement amplification } \\ \text { SD } & \text { Standard Deviation } \\ \text { STD } & \text { Sexually Transmitted Disease } \\ \text { STI } & \text { Sexually Transmitted Infection } \\ \text { TPHA } & \text { Treponema pallidum haemagglutination } \\ \text { WHO } & \text { World Health Organization }\end{array}$




\section{Contents}

Chapter 1

General introduction

14

\section{Section test policy, test evaluation}

Chapter 2 The added value of chlamydia screening between 2008-2010 in reaching young people in addition to chlamydia testing in regular care; an observational study

BMC Infect Dis 2014; 14:612

Chapter 3 Who tests whom? A comprehensive overview of Chlamydia trachomatis test practices in a Dutch region among different STI care providers for urogenital, anorectal and oropharyngeal sites in young people: a cross-sectional study Sex Transm Infect 2015 aug 11. doi:10.1136/sextrans-2015-052065

\section{Section extra-genital testing}

Chapter $4 \quad$ Standard symptom- and sexual history-based testing misses anorectal Chlamydia trachomatis and Neisseria gonorrhoeae infections in swingers and men who have sex with men Sex Transm Dis 2013; 40(4):285-9

Chapter 5 Evaluation of the anatomical site distribution of chlamydia and gonorrhoea in men who have sex with men and in high-risk women by routine testing: cross-sectional study revealing missed opportunities for treatment strategies

Sex Transm Infect 2014 ;90(1):58-60 
Chapter 6 High co-occurrence of anorectal chlamydia with urogenital chlamydia in women visiting an STI clinic revealed by routine universal testing in an observational study; a recommendation towards a better anorectal chlamydia control in women BMC Infect Dis 2014 May 19;14:274

Chapter $7 \quad$ Prevalence of and factors associated with rectal-only anorectal chlamydia and gonorrhoea in women and in men who have sex with men

PLoS ONE 2015 oct 29; 10(10). doi:10.1371/journal.pone.0140297

\section{Section bacterial load}

Chapter 8 Anorectal Chlamydia trachomatis load is similar in men who have sex with men and women reporting anal sex PLoS ONE 2015 aug 11;10(8). doi:10.1371/journal.pone.0134991

Chapter 9

General discussion 



\section{"The secret of happiness, you see, is not found in seeking more, but in developing the capacity to enjoy less.}

Socrates 



\section{General}

introduction 


\section{Background}

Chlamydia trachomatis (CT) is the most prevalent bacterial sexually transmitted infection (STI) worldwide, both among high risk groups and the general population. The Centre of Disease Control (CDC) reported about 1.5 million new infections in 2011 and numbers are still increasing each year[1]. In Europe, the number of CT infections is increasing as well; the ECDC reports more than 250000 new cases each year[2]. CT infections can result in serious sequelae such as pelvic inflammatory disease (PID), ectopic pregnancy and infertility in women, and urethritis and epididymitis in men[3]. Moreover, CT infection can facilitate human immunodeficiency virus (HIV) transmission[4].

Besides the urogenital tract, CT can also cause anorectal and oropharyngeal infections. The majority of CT infections are asymptomatic; $50-80 \%$ of urogenital infections [5,6], 50-90\% of anorectal infections[6-8] and $98-100 \%$ of oropharyngeal infections[6,9-11]. The asymptomatic nature of CT makes it hard to target infections in practice. CT control strategies are mainly focused on primary prevention by sexual education and secondary prevention by active testing and treatment[1]. Internationally, five major strategies are used for the prevention and control of STIs provided by the Centre of Disease Control (CDC) in the US:

- Accurate risk assessment, education and counselling of individuals at risk on ways to avoid STIs through changes in sexual behaviours and use of recommended prevention services.

- Pre-exposure vaccination of individuals at risk for vaccine-preventable STIs.

- Evaluation, treatment, and counselling of sex partners of individuals who are infected with an STI.

- Identification of asymptomatically infected individuals and of individuals with symptoms associated with STIs.

- Effective diagnosis, treatment, counselling and follow up of infected individuals.

This thesis focusses on the latter two strategies by an evaluation of the effectiveness of the current procedures to control CT, i.e., the current testing practices and guidelines, and will provide recommendations for future policy.

\section{Chlamydia trachomatis bacterium}

CT is a gram negative intracellular bacterium. Up to now, nineteen CT serovars have been identified. Serovars A-C mainly cause ocular infections, D-K mainly cause anogenital infections, and the serovars L1-L3 cause the disease lymphogranuloma venereum (LGV)[12,13]. After sexual transmission, the elementary body (EB) form of the bacteria attaches and invades host-epithelial cells. Within the cell, the EBs fuse to form a nascent inclusion. Within the inclusion, the EB transforms into an intracellular form, a reticulate body (RB). The RBs are the metabolically 
active and replicative form of the pathogen. RBs replicate by binary fission and transform back into EB form. The formed EBs are eventually released by exocytosis or host cell lysis[14]. The life cycle of CT is shown in figure 1.

Figure 1: The life cycle of CT adapted from Margaret R. Hammerschlag in Pediatrics in Review 2004;25:43-51. N=nucleus, $\mathrm{EB}=$ elementary body, RB=reticulate body

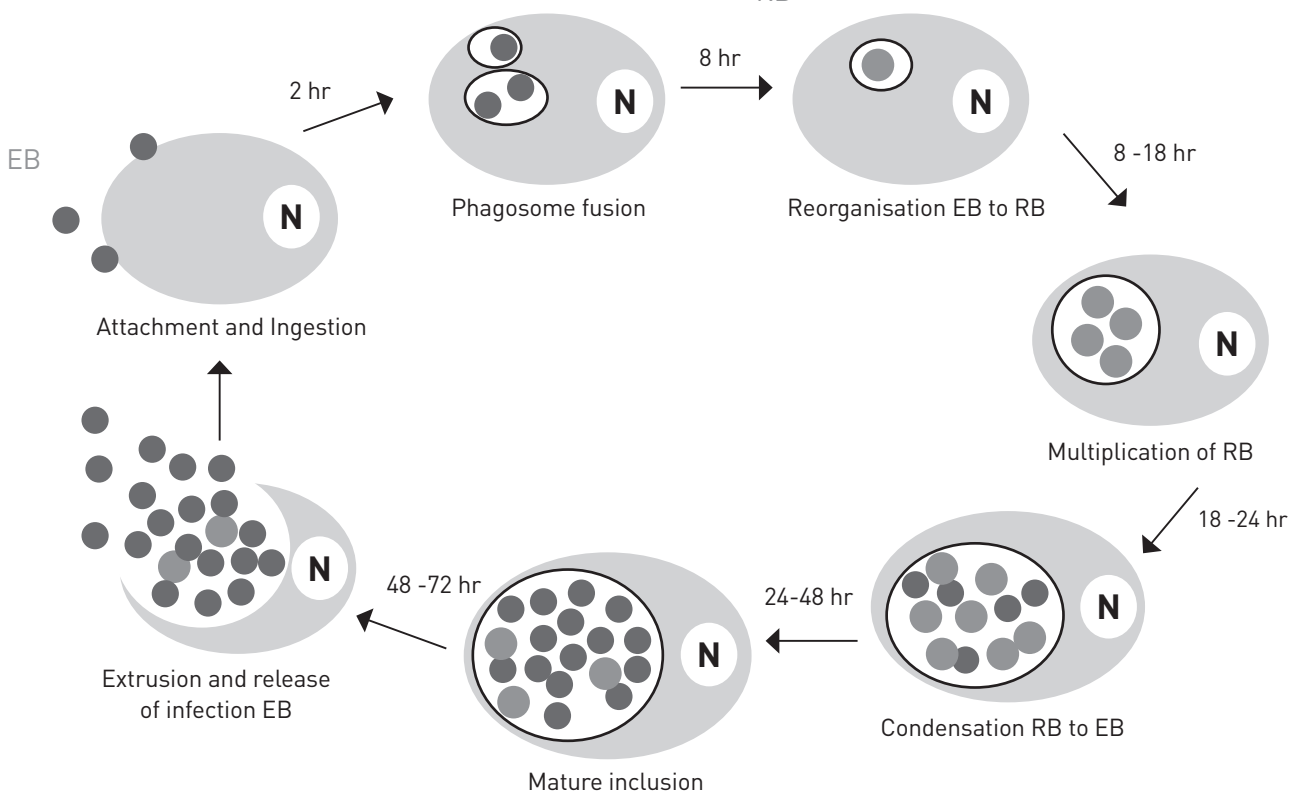

\section{CT Transmission}

CT can be transmitted from mother to child or through sexual contact[1]. The incubation period is usually between 7-14 days, depending on the diagnostic test used[15]. Urogenital CT transmission depends on the number of sex partners and the number of unprotected sex acts within a sexual partnership[16]. CT transmission can occur during vaginal sex but also during anal sex. Transmission from the oropharynx to the urethra is another option, however, the transmission probabilities per sex act are yet unknown[17]. Moreover, alternative transmission routes, for example, fingers and toys, could possibly also play a role for both urogenital and anorectal CT infections. The transmission ability of CT could be influenced by bacterial factors such as virulence[18] and tissue tropism[13] or host factors such as an immune response[19] and coinfections with for example HIV[20]. 


\section{The natural history of CT infection}

Some CT infections clear without therapy, however these infections could have led to transmission. Insight in the natural history of CT infections in men and women could be useful to determine screening and rescreening intervals, timing of notification of test results and treatment, and optimizing partner notification and treatment recommendations[21]. However, it is an ethical challenge to study the natural history of CT infections in humans. Only one study from Colombia collected prospective data of urogenital CT infections $>1$ year without treatment and reported that $54 \%$ of the infections spontaneously cleared at 1 year[5]. Other studies used the time between testing and treatment and reported urogenital CT clearance in women between 11-44\%[22-26], which suggests some degree of protective immunity[21]. Studies looking into the natural history of anorectal and oropharyngeal infections are scarce. The only study published also uses the time between testing and treatment and reported clearance of CT infection in $18 \%(2 / 11)$ of anorectal infections and $50 \%$ (1/1) of oropharyngeal infections[27].

\section{Diagnosis and treatment}

CT testing has become widespread since the introduction of nucleic acid amplification tests (NAATs), which can also be used for the detection of other STI like Neisseria gonorrhoea in the same sample[28]. Since their introduction in the early 90s, NAATs are considered to be the golden standard for urine, cervical swab and for vaginal swab specimens because of high sensitivity and specificity[1]. The performance of NAAT on self-collected vaginal swab specimens is at least as good as other approved specimens such as clinician taken swabs of the endocervix, and this method is highly accepted by women[29]. NAATs are already widely used for anorectal and oropharyngeal testing, despite the absence of FDA clearance[6]. However, anorectal and oropharyngeal testing is performed rarely, especially in women, even when anal sex is reported[30]. Once CT infection is diagnosed using NAATs, subsequent treatment follows. Urogenital and oropharyngeal CT infections are treated with single-dose azithromycin, which is the first choice treatment in the US, the UK and the Netherlands[1,31,32]. Uncomplicated anorectal CT infections are treated with a 7-day course of doxycycline in the Netherlands[32]. In the UK and US, azithromycin is considered an equal treatment for uncomplicated anorectal CT[1,31].

\section{Clinical manifestations urogenital CT}

Clinical studies focus on the urogenital area and show that CT primarily replicates within the epithelium of the urethra of men and urethra and endocervix of women causing inflammation, 
edema, and mucosal discharge[14]. Nevertheless, the majority (80\%) of urogenital CT infections in women are asymptomatic[5]. In women, a possible complication of urogenital CT is pelvic inflammatory disease (PID), where CT ascends to the upper genital tract causes infection and inflammation of the uterus, fallopian tubes, and ovaries. Eventually this infection could cause tubal factor infertility, ectopic pregnancy, chronic pelvic pain and infertility[33,34]. However, the incidence and progression of PID and long term sequelae from an untreated CT infection have not been fully determined[35]. Therefore, prospective studies assessing the progression rates of both PID and reproductive sequelae among women acquiring (repeated) CT infection would be useful to better understand the long term risks of urogenital CT infection[35]. However, designing such studies would be an ethical challenge.

In men, about half of CT infected individuals report symptoms, when symptoms are systematically asked for[3]. The most reported clinical symptom is urethritis, i.e., abnormal urethral discharge, painful or frequent urination and epididymitis[36]. It is controversial whether or not CT infection has adverse effects in men like prostatitis and male fertility[37].

\section{Clinical manifestations extra-genital CT}

Extra-genital CT infections, that is, anorectal and oropharyngeal infections, have the highest proportion asymptomatic infections (up to $90-100 \%$ respectively)[6-10]. Pharyngitis could be a complication of oropharyngeal CT. However, this is reported infrequently and none of the individuals who reported pharyngitis had oropharyngeal CT/NG in a study among STI clinic attendees in the Netherlands[9]. Proctitis, i.e. anal itching, pain or discharge, could be a complication of anorectal CT[1,38]. Moreover anorectal CT could facilitate HIV transmission[7]. Previous studies did not find an association between anal symptoms and anorectal CT[7,9,10,30]. This accounts only for non-lymphogranuloma venereum (LGV) anorectal CT infections. LGV is an invasive ulcerative STI caused by CT biovar L. LGV can cause an anorectal syndrome characterized by severe proctitis with anal cramps (tenesmus), pain, bloody discharge, and constipation caused by local edema[39]. In women, LGV is very unusual, although systematic assessment of LGV in women is scarce.

\section{International CT testing guidelines}

Early detection of STI is an important feature of effective control efforts. This will result in both individual health gains by preventing sequelae due to treatment, and public health gains by preventing onward transmission into the population[40]. International guidelines are provided by the CDC in the US. In the UK, the British Association for Sexual Health (BASHH) provides STI guidelines in cooperation with the Clinical Effectiveness Group [CEG][31,41,42]. In the Nether- 
lands, testing guidelines for general practitioners (GPS) in primary care are provided by the Dutch GP association (NHG)[43]. Testing guidelines for the STI clinics are provided by STI AIDS Netherlands in association with the Dutch association of venereology and dermatology (NVDV) [32]. A summary of both international and national guidelines is provided in table 1.

Table 1: Brief overview of national and international CT testing guidelines

\begin{tabular}{|c|c|c|c|c|}
\hline & US & UK & the Netherlands & \\
\hline & CDC & BASHH-CEG & GPs & STI clinics \\
\hline Women & $\begin{array}{l}\text { Annual screening } \\
\text { of all sexually active } \\
\text { women < } 25 \text { years. } \\
\text { Older women with risk } \\
\text { behaviour such as } \\
\text { having a new sex partner } \\
\text { or multiple sex partners. }\end{array}$ & $\begin{array}{l}\text { Asymptomatic } \\
\text { individuals based on } \\
\text { individual risk for } \\
\text { infection. }\end{array}$ & $\begin{array}{l}\text { Youngsters are } \\
\text { considered to be at } \\
\text { higher risk for CT. }\end{array}$ & $\begin{array}{l}\text { Youngsters who are } \\
\text { recently sexually active } \\
\text { are considered to be at } \\
\text { high risk for acquiring } \\
\text { urogenital CT. }\end{array}$ \\
\hline $\begin{array}{l}\text { Heterosexual } \\
\text { men }\end{array}$ & $\begin{array}{l}\text { No routine urogenital } \\
\text { CT testing in sexually } \\
\text { active heterosexual } \\
\text { young men. } \\
\text { Consider testing sexually } \\
\text { active young men in } \\
\text { clinical settings with high } \\
\text { prevalence of CT (for } \\
\text { example, adolescent } \\
\text { clinics, correctional } \\
\text { facilities, and STD clinics). }\end{array}$ & $\begin{array}{l}\text { Asymptomatic } \\
\text { individuals based on } \\
\text { individual risk for } \\
\text { infection. }\end{array}$ & $\begin{array}{l}\text { Youngsters are } \\
\text { considered to be at } \\
\text { higher risk for CT. }\end{array}$ & $\begin{array}{l}\text { Youngsters who are } \\
\text { recently sexually } \\
\text { active are considered } \\
\text { to be at high risk for } \\
\text { acquiring urogenital CT. }\end{array}$ \\
\hline Extra-genital & $\begin{array}{l}\text { Anorectal / } \\
\text { oropharyngeal CT } \\
\text { testing in individuals } \\
\text { who report receptive } \\
\text { anal intercourse/ } \\
\text { receptive oral } \\
\text { intercourse } \\
\text { respectively. }\end{array}$ & $\begin{array}{l}\text { Insufficient evidence } \\
\text { to advice anorectal } \\
\text { testing in heterosexual } \\
\text { men and women. } \\
\text { Anorectal testing in } \\
\text { CSW's who report } \\
\text { sexual behaviours } \\
\text { which may result in } \\
\text { rectal infection. }\end{array}$ & $\begin{array}{l}\text { Based on sexual } \\
\text { history and reported } \\
\text { symptoms, anorectal } \\
\text { and/or oropharyngeal } \\
\text { testing should be } \\
\text { considered. }\end{array}$ & $\begin{array}{l}\text { Anorectal CT testing in } \\
\text { individuals who report } \\
\text { receptive anal } \\
\text { intercourse. }\end{array}$ \\
\hline $\begin{array}{l}\text { MSM including } \\
\text { extra-genital }\end{array}$ & $\begin{array}{l}\text { Urogenital testing after } \\
\text { insertive intercourse, } \\
\text { anorectal testing after } \\
\text { receptive anal } \\
\text { intercourse and } \\
\text { oropharyngeal testing } \\
\text { after receptive oral } \\
\text { intercourse, all regarding } \\
\text { the preceding year. }\end{array}$ & $\begin{array}{l}\text { Anorectal testing in } \\
\text { MSM who report } \\
\text { sexual behaviours } \\
\text { which may result in } \\
\text { rectal infection. }\end{array}$ & $\begin{array}{l}\text { MSM are considered to } \\
\text { be at higher risk for CT. } \\
\text { Extra-genital testing } \\
\text { based on sexual } \\
\text { history and reported } \\
\text { symptoms. }\end{array}$ & $\begin{array}{l}\text { MSM are considered } \\
\text { to be at high risk for CT. } \\
\text { Anorectal CT testing } \\
\text { in individuals who } \\
\text { report receptive anal } \\
\text { intercourse. }\end{array}$ \\
\hline Retesting & $\begin{array}{l}\text { CT infected men and } \\
\text { women approximately } \\
3 \text { months after } \\
\text { treatment. } \\
\text { In case this is not } \\
\text { possible, CT infected } \\
\text { individuals should be } \\
\text { retested in the } 12 \\
\text { months after initial } \\
\text { treatment, when they } \\
\text { attend medical care. }\end{array}$ & $\begin{array}{l}\text { No specific } \\
\text { recommendations } \\
\text { for retesting are } \\
\text { made, except in young } \\
\text { individuals with a } \\
\text { history of NG; retesting } \\
\text { is recommended, } \\
\text { however the interval } \\
\text { has not been defined. }\end{array}$ & $\begin{array}{l}\text { No statements are } \\
\text { included about } \\
\text { retesting (CT positive) } \\
\text { individuals. }\end{array}$ & $\begin{array}{l}\text { No statements are } \\
\text { included about } \\
\text { retesting (CT positive) } \\
\text { individuals. }\end{array}$ \\
\hline
\end{tabular}




\section{Guidelines and policy in the Netherlands}

In the Netherlands, a policy of proximity and easy access to healthcare has been implemented to promote sexual health and to control transmission. Regular care for testing and treatment of CT is provided by GPs, STI-clinics and after referral by medical specialists (mainly gynaecologists)[44]. GPs are the core primary care-provider and all inhabitants are registered in a general practice[45]. The role of the GP in CT testing is to focus on the general population. STI clinics are subsidized by the Ministry of Health to maintain free anonymous low threshold care to risk groups. Individuals are considered to belong to a risk group if they (1) report STI related symptoms, (2) are notified or referred for STI testing, (3) are aged below 25 years, (4) are men who have sex with men (MSM), (5) are involved in commercial sex, (6) originate from an STI/HIV endemic country, (7) report three or more sexual partners in the past 6 months or (8) report a partner from one of these risk groups. Up to 2012, all attendees were tested for CT, NG, and syphilis on a mandatory base. Since 2012 , youngsters $<25$ years who do not belong to another risk group are only tested for CT. In case the test is positive, further testing for gonorrhoea, syphilis and HIV will be offered[32]. For HIV testing there is an opt-out policy[46]. However, when receptive anal sex is reported, blood tests for syphilis, hepatitis B and HIV are routinely performed[47]. STI clinics also offer extra-genital tests for example to MSM. It is assumed that GPS offer extra-genital tests rarely to patients. Besides testing, the STI clinic also provides free sex counselling and education, for example on sexual problems, contraception and pregnancy. Trained STI nurses also provide partner notification, in case accepted by the client this could be provider notification or patient notification.

\section{CT screening}

In 2002, England implemented the National Chlamydia Screening Programme (NCSP) which uses opportunistic screening in primary care. Young people (15-24 years) are offered a CT test when attending care, irrespective of reason for attending[48]. Annual coverage of the NCSP is used to assess local delivery of this screening programme[49]. In 2010/20112.2 million CT tests were performed in England, which is about 43\% coverage. This was a $9 \%$ increase compared to the previous year[50]. Of these 2.2 million tests, nearly 1.4 million were performed by the NCSP. A mathematical model on the effectiveness of CT screening in England suggested that an opportunistic screening programme could reduce CT prevalence within a few years by offering screening to the entire eligible population of young people. However, this reduction in CT prevalence depends on regular attendance at healthcare providers, and high quality data on healthcare attendance is lacking[51]. 
In the Netherlands, systematic population-based internet urogenital CT screening was initiated in 2008 in Amsterdam, Rotterdam and South-Limburg, in addition to regular care. This registerbased internet enhanced urogenital CT screening program (CSI) aimed to improve case finding, prevent sequelae and reduce population prevalence by annual testing and treatment of people aged 16-29 years[52]. In South Limburg, eligibility for urogenital CT testing within the CT screening programme depended on an individual's CT risk score. This risk score was based on answers to an eight-item risk questionnaire (i.e. age, place of residence, education level, condom use at last intercourse, number of lifetime sex contacts, ethnic background, having a new sexual partner in the last 6 months and symptoms)[53]. When an individual was eligible, home sampling kits for urogenital testing (urine or vaginal swab) could be requested through a website (www.chlamydiatest.nl). Treatment and partner notification were done by the GP or at a STI clinic[54]. In the first screening round, 261,025 invitations were send. Within 4 weeks after the invitation was send, $11.9 \%$ requested a test kit. The remaining invitees were reminded after which an additional $8.3 \%$ requested a test kit. A total of 52,741 kits were requested upon invitation. In the urban areas the overall request rate was $20.5 \%$, while in rural South Limburg this was $13.8 \%$, possibly due to the dependency on CT risk score. A total of $22.5 \%$ filled in the online risk score and $63 \%$ were eligible. Overall urogenital CT positivity was $4.2 \%$, this was significantly higher among women compared to men ( $4.4 \%$ versus $3.8 \%)$ and higher among young individuals ( $<20$ years $7.3 \%$ versus $3.8 \%$ ). Especially young girls tested positive for urogenital CT more often (8\%)[52]. Anorectal and oropharyngeal CT testing was not included in CT screening programs in both the UK and the Netherlands. Participation rates in CT screening in the Netherlands were relative low and for each screening round fewer participants were encountered. It is not known whether these participants were already tested for CT by regular health care providers. They might have been already familiar with STI care, or participants might have been previously untested, revealing a hidden CT risk population.

\section{Epidemiology of CT in the Netherlands}

GPs and STI clinics have different systems for data registration. GPs use electronic medical records (EMRs) with ICPC-codes (International Classification of Primary Care) for health problems. However, a specific code for each STI test is lacking. For surveillance and studies, the definition of $\mathrm{CT}$ is based on a combination of several codes and antibiotic prescriptions. Examples of such codes are vaginitis, cervicitis, or PID in women and orchitis/epididymitis or 'other genital diseases' in men[55]. Sex and test result are available in the GP database, however anatomic site of testing and sexual behaviour are lacking. Prevalence data of GPs are calculated using general practices participating in NIVEL Primary Care Database (NIVEL-PCD), which is a representative sample of the Dutch population. The NIVEL monitors health and utilisation of health services by 
using routinely recorded data from health care providers.

A fourfold increase of the number of participating general practices in NIVEL-PCD in 2012 has led to changes in definitions of episodes and in calculations of prevalence (i.e. more specific selection of patient years), which caused an increase in nearly all STI numbers.

STI clinics in the Netherlands also use an electronic database for patients' registration. The national STI surveillance is organised in 8 Dutch Sexual Healthcare regions, with one coordinating STI centre (part of a Public Health Service or 'GGD') per region. Data from all clinics nationwide are reported to a register of the National Centre for Infectious Disease Control (Clb) which is a part of the National Institute for Public Health and the Environment (RIVM). The Clb maintains the national STI surveillance (SOAP). In contrast to GP data, STI clinic data include anatomic site of testing and more extensive data on sexual behaviour.

Up to 2010, the majority of patients with STI-related problems were seen in primary care. A study among 75 GP practices in 2001 estimated 98.000 episodes related to STI/HIV care, in contrast to 38.000 episodes in STI clinics[45]. In 2001, the Dutch National Survey of General Practice included questions about STI and health seeking behaviour. The aim of these large national surveys is to obtain information on health status, health-care utilization and quality of primary care. Of all individuals, aged 18 years and older, reporting STI related symptoms $62 \%$ visited the GP, followed by $20 \%$ who visited an STI clinic[45]. From 2010, the ratio CT tests between GPs and STI centres is about 50/50, with approximately 100.000 tests in 2010 by each provider. The proportion men/women tested by GPs and STI clinics was comparable (52\%/48\%). In STI clinics, 33\% were heterosexual males and 19\% were MSM, this is unknown for GPs. The overall CT positivity rates were also comparable between GPs and STI centres $(15 \%)[55,56]$.

In 2013, the total number of consultations at STI clinics in the Netherlands was 133.585, which was a $10 \%$ increase compared to 2012 . Most consultations in 2013 were by youngsters $<25$ years of age (49\%), followed by individuals who originate from an STI/HIV endemic country (25\%) and MSM (21\%). CT was the most diagnosed STI in 2013 with 15.767 new infections and a positivity rate of $11.8 \%$, which was slightly lower compared to 2012 (12.2\%). Prevalence of CT was $12.8 \%$ in heterosexual men, $9.6 \%$ in MSM and $12.2 \%$ in women. The largest proportion of CT was diagnosed among youngsters $<25$ years of age $(61 \%)$. Youngsters aged $15-19$ years had the highest positivity rate; $19.7 \%$ in women and $17.1 \%$ in men. Individuals who reported a previous STI (NG, CT and/or syphilis) and known HIV-positive MSM were also at high risk for CT (16.2\% and 15.9\% respectively). Native Dutch STI-clinic attendees were the largest group tested, and the majority of CT cases were diagnosed in this group. STI-clinic attendees of Surinamese or Dutch Antillean descent had the highest overall positivity rate (15.3 and 17.8 per cent respectively). In total, 16.301 youngsters $<25$ years who did not belong to an other risk group were tested for CT only, $7.8 \%$ tested positive and were further tested for other STI. Among these CT positives, 1.1\% was positive for NG, and all were tested negative for syphilis and HIV. The total number of tests and 
Figure 2: Total number of tests and positivity rate of CT by gender and sexual preference by STI clinics in the Netherlands, 2004-2013

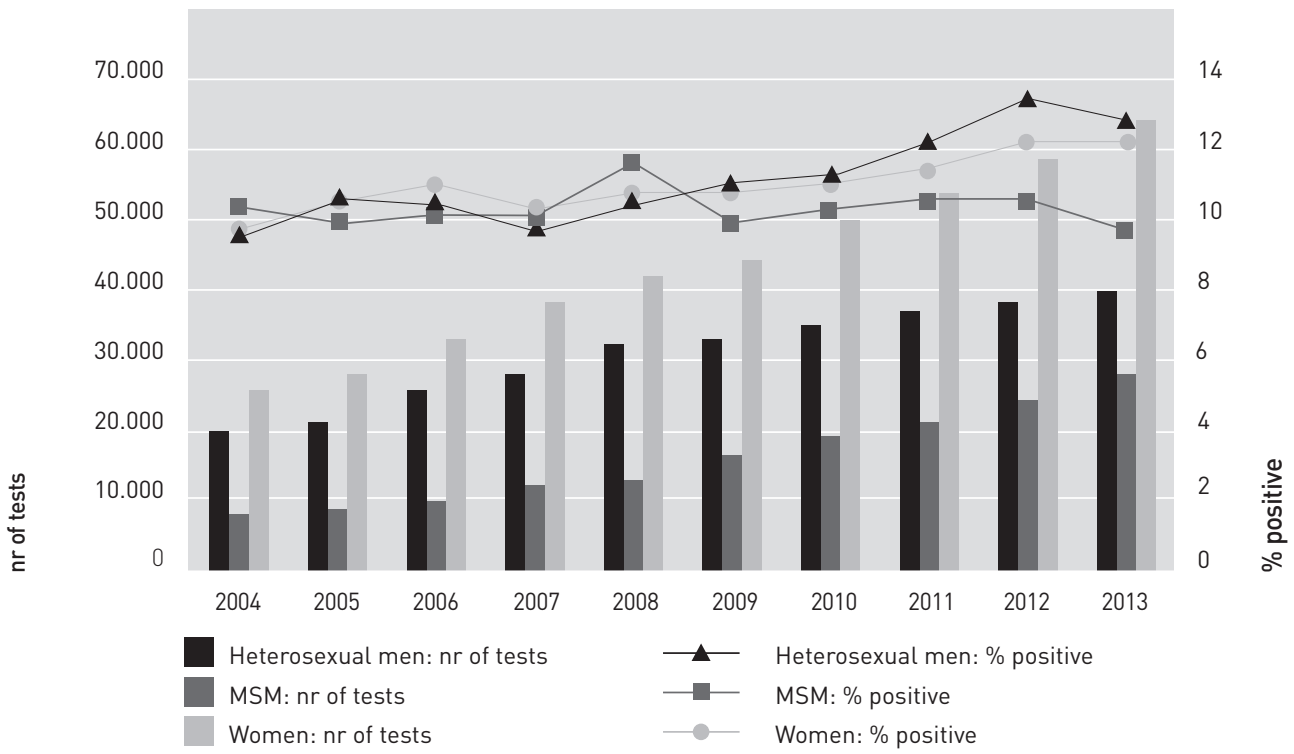

Source: van Aar F, Koedijk FDH, van den Broek IVF, Op de Coul ELM, Soetens LC, Woestenberg PJ, Heijne JCM, van Sighem Al, Nielen MMJ, van Benthem BHB. "Sexually transmitted infections in the Netherlands in 2013".

Figure 3: Estimated prevalence of episodes of CT at GPs by gender, based on extrapolation from practices in the surveillance network of NIVEL-PCD, using the old method (2002-2011) and the new method (2010-2012)

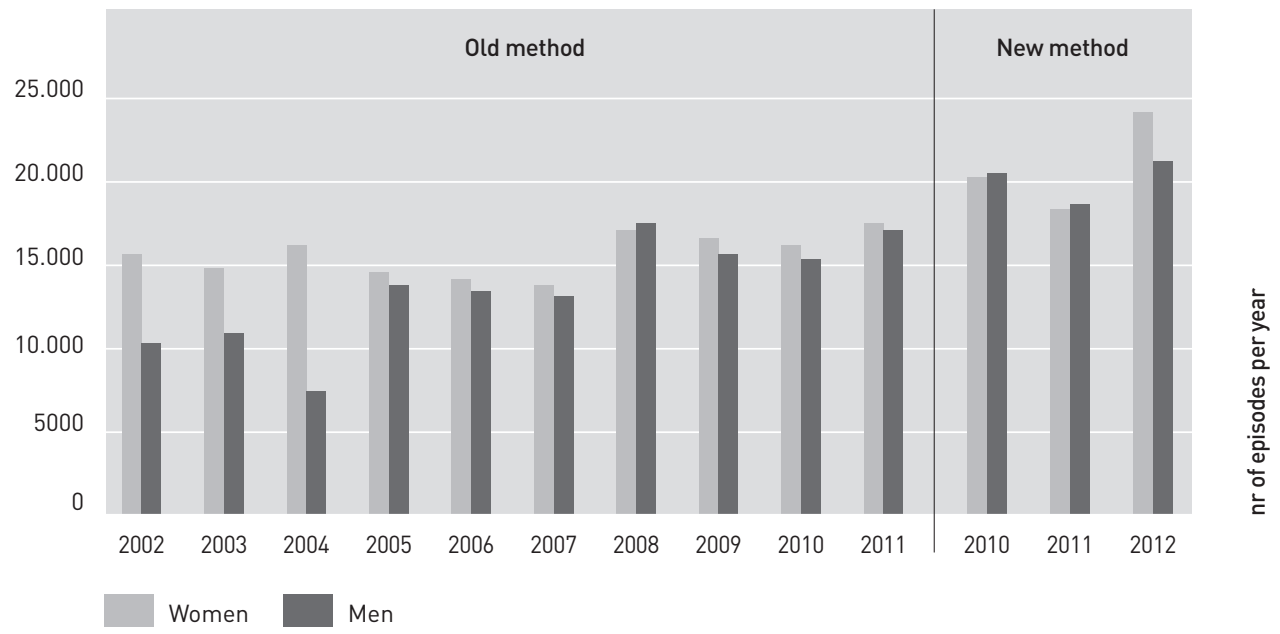

Source: van Aar F, Koedijk FDH, van den Broek IVF, Op de Coul ELM, Soetens LC, Woestenberg PJ, Heijne JCM, van Sighem Al, Nielen MMJ, van Benthem BHB. "Sexually transmitted infections in the Netherlands in 2013". 
positivity rate of CT by sex and sexual preference in STI clinics in the Netherlands from 20042013 is presented in figure 2 .

GP data show that the total number of STI diagnoses and STI related episodes is higher compared to the STI clinics. GPs also reach high risk groups such as heterosexual youngsters $<25$ years of age and individuals who originate from an STI/HIV endemic countries. However, fewer specific groups such as MSM and commercial sex workers (CSWs) are reached by GPs.

The estimated number of CT episodes at GPs was 273 per 100.000 inhabitants in 2012. An estimated 46.000 cases of CT were diagnosed at GPs annually between 2010-2012 (figure 3). The estimated number of CT cases between 2010-2012 was calculated using the new method, which has led to an increase in the number of cases. This is probably due to changes in definitions. The estimated prevalence of CT episodes at GPs, by sex is presented in figure 3.

\section{Extra-genital infections}

STI clinics distinguish CT infections by different anatomic sites. Focus of (international) guidelines is on urogenital CT testing. However, national data show that prevalence of extra-genital CT, i.e., anorectal and oropharyngeal CT, is also substantial among MSM and women. In women and heterosexual men in 2013, prevalence of urogenital CT is highest with $11.5 \%$ and $12.7 \%$ respectively. In MSM prevalence of anorectal CT is higher compared to urogenital and oropharyngeal CT $(8.2 \%$ versus $3.5 \%$ and $1.1 \%)$. However, prevalence of anorectal CT is also substantial in women $(10.2 \%)$ and anorectal CT was also found among heterosexual men ( $n=25)$ (table 2).

\section{Aim of this thesis}

The continuing high proportion of CT positives indicates that there are still challenges in STI control. Despite various control measures, such as testing guidelines, CT screening and optimized delivery of (free, anonymous) STI care, CT testing and prevalence rates are continuing to rise. Current gaps in knowledge include the distribution of CT tests and positivity per CT care provider. Who tests whom and how can this be optimised? If care providers follow the guidelines, would that be optimal CT care, or would there be CT infections missed? For example with current worldwide selective testing for extra-genital CT, in contrast to routine universal urogenital testing. This thesis focusses on an evaluation of the effectiveness of current testing practices and guidelines to control CT and will provide recommendations for future policy.

The research in this thesis is the result of collaboration between the Public Health Service South Limburg (Academische Werkplaats Limburg) and the department of Medical Microbiology Maastricht University Medical centre within rersearch school CAPHRI to connect research, 
policy and practice. Further collaborations include the Public Health Service Amsterdam, Public Health Service Rotterdam, the National Institute for Public Health and the Environment (RIVM), and STI/AIDS Netherlands. The public health gain of this collaboration is a more evidence-based practice by state of the art research which focusses on current challenges in care.

Table 2: Number and percentage of positive tests for CT by anatomic location, gender and sexual preference by STI clinics in the Netherlands, 2007-2013. Adapted from; van Aar F, Koedijk FDH, van den Broek IVF, Op de Coul ELM, Soetens LC, Woestenberg PJ, Heijne JCM, van Sighem Al, Nielen MMJ, van Benthem BHB. "Sexually transmitted infections in the Netherlands in 2013"

\begin{tabular}{|c|c|c|c|c|c|c|}
\hline & $\begin{array}{l}2008 \\
\text { n positive } \\
{[\%]}\end{array}$ & $\begin{array}{l}2009 \\
\text { n positive } \\
(\%)\end{array}$ & $\begin{array}{l}2010 \\
n \text { positive } \\
(\%)\end{array}$ & $\begin{array}{l}2011 \\
\text { n positive } \\
(\%)\end{array}$ & $\begin{array}{l}2012 \\
\text { n positive } \\
(\%)\end{array}$ & $\begin{array}{l}2013 \\
\text { n positive } \\
(\%)\end{array}$ \\
\hline \multicolumn{7}{|c|}{ Heterosexual men } \\
\hline Urogenital & $\begin{array}{l}3343 \\
(10.6)\end{array}$ & $\begin{array}{l}3480 \\
(10.8)\end{array}$ & $\begin{array}{l}3922 \\
(11.3)\end{array}$ & $\begin{array}{l}4434 \\
(11.9)\end{array}$ & $\begin{array}{l}5052 \\
(13.2)\end{array}$ & $\begin{array}{l}5154 \\
(12.7)\end{array}$ \\
\hline Anorectal & $\begin{array}{l}2 \\
(0.8)\end{array}$ & $\begin{array}{l}7 \\
(1.6)\end{array}$ & $\begin{array}{l}13 \\
(2.8)\end{array}$ & $\begin{array}{l}17 \\
(2.8)\end{array}$ & $\begin{array}{l}22 \\
\text { (3.3) }\end{array}$ & $\begin{array}{l}25 \\
(2.9)\end{array}$ \\
\hline Oral & $\begin{array}{l}6 \\
(1.1)\end{array}$ & $\begin{array}{l}4 \\
(0.5)\end{array}$ & $\begin{array}{l}10 \\
(1.0)\end{array}$ & $\begin{array}{l}11 \\
(0.8)\end{array}$ & $\begin{array}{l}18 \\
(1.3)\end{array}$ & $\begin{array}{l}20 \\
(1.2)\end{array}$ \\
\hline \multicolumn{7}{|l|}{ MSM } \\
\hline Urogenital & $\begin{array}{l}651 \\
(4.8)\end{array}$ & $\begin{array}{l}661 \\
(4.1)\end{array}$ & $\begin{array}{l}790 \\
(4.1)\end{array}$ & $\begin{array}{l}852 \\
(3.9)\end{array}$ & $\begin{array}{l}875 \\
(3,6)\end{array}$ & $\begin{array}{l}954 \\
(3.5)\end{array}$ \\
\hline Anorectal & $\begin{array}{l}1046 \\
(11.7)\end{array}$ & $\begin{array}{l}1081 \\
(9.5)\end{array}$ & $\begin{array}{l}1381 \\
(9.5)\end{array}$ & $\begin{array}{l}1537 \\
(9.1)\end{array}$ & $\begin{array}{l}1800 \\
(9.1)\end{array}$ & $\begin{array}{l}1856 \\
(8.2)\end{array}$ \\
\hline Oral & $\begin{array}{l}72 \\
(2.1)\end{array}$ & $\begin{array}{l}81 \\
\text { (1.5) }\end{array}$ & $\begin{array}{l}134 \\
(1.8)\end{array}$ & $\begin{array}{l}218 \\
(1.2)\end{array}$ & $\begin{array}{l}272 \\
(1.3)\end{array}$ & $\begin{array}{l}264 \\
(1.1)\end{array}$ \\
\hline \multicolumn{7}{|l|}{ Women } \\
\hline Urogenital & $\begin{array}{l}4385 \\
(10.3)\end{array}$ & $\begin{array}{l}4521 \\
(10.3)\end{array}$ & $\begin{array}{l}5386 \\
(10.7)\end{array}$ & $\begin{array}{l}6109 \\
(11.4)\end{array}$ & $\begin{array}{l}6736 \\
(11.6)\end{array}$ & $\begin{array}{l}7498 \\
(11.5)\end{array}$ \\
\hline Anorectal & $\begin{array}{l}328 \\
(9.4)\end{array}$ & $\begin{array}{l}380 \\
(9.2)\end{array}$ & $\begin{array}{l}439 \\
(9.2)\end{array}$ & $\begin{array}{l}551 \\
(9.3)\end{array}$ & $\begin{array}{l}740 \\
(9.5)\end{array}$ & $\begin{array}{l}1028 \\
(10.2)\end{array}$ \\
\hline Oral & $\begin{array}{l}134 \\
(2.3)\end{array}$ & $\begin{array}{l}214 \\
(2.9)\end{array}$ & $\begin{array}{l}239 \\
(2.6)\end{array}$ & $\begin{array}{l}416 \\
(2.8)\end{array}$ & $\begin{array}{l}586 \\
(3.4)\end{array}$ & $\begin{array}{l}719 \\
(3.3)\end{array}$ \\
\hline
\end{tabular}

Heterosexual men and women are not often tested anorectally or orally, therefore the fluctuation of positivity rates through the years has to be interpreted with caution. Please note that people can have positive tests at multiple locations. 


\section{Outline of this thesis}

The aim of this thesis is to optimize CT control strategies by evaluating of the effectiveness of the current testing practices and guidelines. Several strategies have been implemented to control CT, for example a population based CT screening programme among young people. Chapter 2 assesses the added value of this screening programme to reach young people in addition to regular care. Chapter 3 provides an overview of CT testing practices among young people in one geographical region. The key question in this chapter is 'Who tests whom?', with respect to the distribution of CT tests per test provider, positivity rates and a range of demographics.

Current guidelines advocate selective symptom- and sexual history-based anorectal testing. This CT control strategy is evaluated in chapter $\mathbf{4}$ using routine systematic testing among two high-risk populations; men who have sex with men (MSM) and swingers. In addition to these high-risk groups, chapter 6 evaluates this strategy in women who visited the STI clinic. To gain further insight in extra-genital infections, chapter $\mathbf{5}$ describes the anatomical site distribution of CT and NG by routine systematic testing in MSM and high-risk women. In addition, chapter $\mathbf{7}$ looks further into rectal-only infections by assessing their prevalence and risk factors in a large group of MSM and women.

Chapter 8 provides insight in anorectal CT infections on a molecular level using bacterial load determination. This chapter compares anorectal CT load between MSM, women who reported anal sex and women who did not report anal sex.

In chapter 9 the main findings of this thesis are discussed and recommendations for policy and future research are provided. 


\section{References}

1. Centers for Disease Control and Prevention, 2011 Sexually Transmitted Diseases Surveillance, MMWR 2010;59:4-6.

2. European Centre for Disease Prevention and Control. Chlamydia control in Europe: literature review. ECDC Stockholm 2014.

3. Eley A, Pacey AA, Galdiero M, Galdiero F. Can Chlamydia trachomatis directly damage your sperm? Lancet Infect Dis. 2005;5: 53-57.

4. Plummer FA, Simonsen JN, Cameron DW, Ndinya-Achola JO, Kreiss JK, Gakinya MN, et al. Cofactors in male-female sexual transmission of human immunodeficiency virus type 1. J Infect Dis. 1991;163: 233-239.

5. Molano M, Meijer CJ, Weiderpass E, Arslan A, Posso H, Franceschi S, et al. The natural course of Chlamydia trachomatis infection in asymptomatic Colombian women: a 5-year follow-up study. J Infect Dis. 2005;191: 907-916.

6. Schachter J, Moncada J, Liska S, Shayevich C, Klausner JD. Nucleic acid amplification tests in the diagnosis of chlamydial and gonococcal infections of the oropharynx and rectum in men who have sex with men. Sex Transm Dis. 2008;35: 637-642.

7. Hunte T, Alcaide M, Castro J. Rectal infections with chlamydia and gonorrhoea in women attending a multiethnic sexually transmitted diseases urban clinic. Int J STD AIDS.

2011;21: 819-822.

8. Twin J, Moore EE, Garland SM, Stevens MP, Fairley CK, Donovan B, et al. Chlamydia trachomatis genotypes among men who have sex with men in Australia. Sex Transm Dis. 2010;38: 279-285.
9. Peters RP, Nijsten N, Mutsaers J, Jansen CL, Morre SA, van Leeuwen AP. Screening of oropharynx and anorectum increases prevalence of Chlamydia trachomatis and Neisseria gonorrhoeae infection in female STD clinic visitors. Sex Transm Dis. 2011;38: 783-787.

10. Peters RP, Verweij SP, Nijsten N, Ouburg S, Mutsaers J, Jansen CL, et al. Evaluation of sexual history-based screening of anatomic sites for chlamydia trachomatis and neisseria gonorrhoeae infection in men having sex with men in routine practice. BMC Infect Dis. 2011;11: 203.

11. Winter AJ, Gilleran G, Eastick K, Ross JD. Comparison of a ligase chain reaction-based assay and cell culture for detection of pharyngeal carriage of Chlamydia trachomatis. J Clin Microbiol. 2000;38: 3502-3504.

12. Morre SA, Rozendaal L, van Valkengoed IG, Boeke AJ, van Voorst Vader PC, Schirm J, et al. Urogenital Chlamydia trachomatis serovars in men and women with a symptomatic or asymptomatic infection: an association with clinical manifestations? J Clin Microbiol. 2000;38: 2292-2296.

13. Bax CJ, Quint KD, Peters RP, Ouburg S, Oostvogel PM, Mutsaers JA, et al. Analyses of multiple-site and concurrent Chlamydia trachomatis serovar infections, and serovar tissue tropism for urogenital versus rectal specimens in male and female patients. Sex Transm Infect. 2011;87: 503-507.

14. Bastidas RJ, Elwell CA, Engel JN, Valdivia RH. Chlamydial intracellular survival strategies. Cold Spring Harb Perspect Med. 2013;3: a010256. 
15. Dommelen Lv. Optimizing Chlamydia trachomatis and Treponema pallidum diagnostics. ISBN: 978-90-9027817-9. 2013.

16. Althaus CL, Turner KM, Schmid BV, Heijne JC, Kretzschmar M, Low N. Transmission of Chlamydia trachomatis through sexual partnerships: a comparison between three individual-based models and empirical data. J R Soc Interface. 2011;9: 136-146.

17. Bernstein KT, Stephens SC, Barry PM, Kohn R, Philip SS, Liska S, et al. Chlamydia trachomatis and Neisseria gonorrhoeae transmission from the oropharynx to the urethra among men who have sex with men. Clin Infect Dis. 2009;49: 1793-1797.

18. Taylor BD, Darville T, Tan C, Bavoil PM, Ness RB, Haggerty CL. The role of Chlamydia trachomatis polymorphic membrane proteins in inflammation and sequelae among women with pelvic inflammatory disease. Infect Dis Obstet Gynecol. 2011: 989762.

19. Yang X, Brunham R. T lymphocyte immunity in host defence against Chlamydia trachomatis and its implication for vaccine development. Can J Infect Dis. 1998;9: 99-108.

20. Sexton J, Garnett G, Rottingen JA. Metaanalysis and metaregression in interpreting study variability in the impact of sexually transmitted diseases on susceptibility to HIV infection. Sex Transm Dis. 2005;32: 351-357.

21. Geisler WM. Duration of untreated, uncomplicated Chlamydia trachomatis genital infection and factors associated with chlamydia resolution: a review of human studies. J Infect Dis. 2010;201 Suppl 2: S104-113.

22. Joyner JL, Douglas JM, Jr., Foster M, Judson
FN. Persistence of Chlamydia trachomatis infection detected by polymerase chain reaction in untreated patients. Sex Transm Dis. 2002;29: 196-200.

23. Morre SA, van den Brule AJ, Rozendaal $L$, Boeke AJ, Voorhorst FJ, de Blok S, et al. The natural course of asymptomatic Chlamydia trachomatis infections: $45 \%$ clearance and no development of clinical PID after one-year follow-up. Int J STD AIDS. 2002;13 Suppl 2: 12-18.

24. Rogers SM, Miller WC, Turner CF, Ellen J, Zenilman J, Rothman R, et al. Concordance of chlamydia trachomatis infections within sexual partnerships. Sex Transm Infect. 2008;84: 23-28.

25. Sheffield JS, Andrews WW, Klebanoff MA, Macpherson C, Carey JC, Ernest JM, et al. Spontaneous resolution of asymptomatic Chlamydia trachomatis in pregnancy. Obstet Gynecol. 2005;105: 557-562.

26. van Valkengoed IG, Morre SA, van den Brule AJ, Meijer CJ, Bouter LM, van Eijk JT, et al. Follow-up, treatment, and reinfection rates among asymptomatic chlamydia trachomatis cases in general practice. Br J Gen Pract. 2002;52: 623-627.

27. Apewokin SK, Geisler WM, Bachmann LH. Spontaneous resolution of extragenital chlamydial and gonococcal infections prior to therapy. Sex Transm Dis. 2010;37: 343-344.

28. Rockett R, Goire N, Limnios A, Turra M, Higgens G, Lambert SB, et al. Evaluation of the cobas $4800 \mathrm{CT} / \mathrm{NG}$ test for detecting Chlamydia trachomatis and Neisseria gonorrhoeae. Sex Transm Infect. 2010;86: 470-473. 
29. van der Helm JJ, Hoebe CJ, van Rooijen MS, Brouwers EE, Fennema HS, Thiesbrummel $H F$, et al. High performance and acceptability of self-collected rectal swabs for diagnosis of Chlamydia trachomatis and Neisseria gonorrhoeae in men who have sex with men and women. Sex Transm Dis. 2009;36: 493-497.

30. Sethupathi M, Blackwell A, Davies H. Rectal Chlamydia trachomatis infection in women. Is it overlooked? Int J STD AIDS. 2009;21: 93-95.

31. The British Association for Sexual Health and HIV. 2006 UK National Guideline for the Management of Genital Tract Infection with Chlamydia trachomatis http://www.bashh. org/documents/65.pdf.

32. HJC de Vries, GJJ van Doornum, CJ Bax et al. Multidisciplinaire Richtlijn Seksueel

Overdraagbare Aandoeningen voor de 2e Lijn http://www.tinyurl.soarichtlijn2012.

33. Cohen CR, Brunham RC. Pathogenesis of Chlamydia induced pelvic inflammatory disease. Sex Transm Infect. 1999;75: 21-24.

34. Paavonen J, Westrom L, Eschenbach DA. Pelvic inflammatory disease. In: Holmes KK, Sparling PF, Stamm WE, et al., eds. Sexually transmitted Paavonen J, Westrom L, Eschenbach DA. Pelvic inflammatory disease. In: Holmes KK, Sparling PF, Stamm WE, et al., eds. Sexually transmitted diseases. New York: McGraw Hill Medical, 2008:1017-1050diseases. New York: McGraw Hill Medical, 2008:1017-1050.

35. Haggerty CL, Gottlieb SL, Taylor BD, Low N, Xu F, Ness RB. Risk of sequelae after Chlamydia trachomatis genital infection in women. J Infect Dis. 2010;201 Suppl 2:

S134-155.
36. Oriel JD, Ridgway GL. Genital infection in men. Br Med Bull. 1983;39: 133-137.

37. Mackern-Oberti JP, Motrich RD, Breser ML, Sanchez LR, Cuffini C, Rivero VE. Chlamydia trachomatis infection of the male genital tract: an update. J Reprod Immunol. 2013;100: 37-53.

38. Hamlyn E, Taylor C. Sexually transmitted proctitis. Postgrad Med J. 2006;82: 733-736.

39. de Vries HJ, Smelov V, Ouburg S, Pleijster J, Geskus RB, Speksnijder AG, et al. Anal lymphogranuloma venereum infection screening with IgA anti-Chlamydia trachomatisspecific major outer membrane protein serology. Sex Transm Dis. 2010;37: 789-795.

40. Peipert JF. Clinical practice. Genital chlamydial infections. N Engl J Med. 2003;349: 2424-2430.

41. Ross J, Ison C, Carder C, Lewis D, Mercey D, Young $\mathrm{H}$ (Screening Guideline Steering Group). Sexually Transmitted Infections: UK National Screening and Testing Guidelines 2006.

42. (2010) Chlamydia trachomatis UK Testing Guidelines 2010. Clinical Effectiveness Group British Association for Sexual Health and HIV.

43. van Bergen JEAM, Dekker JH, Boeke AJP, Mastboom MT, Pijnenborg L, van Lieshout J. NHG-Standaard 'Het soa-consult'. Huisarts Wet. 2005: 636-651.

44. Dukers-Muijrers NH, van Liere GA, Hoebe CJ. Re-screening Chlamydia trachomatis positive subjects: a comparison of practices between an STI clinic, general practitioners and gynaecologists. Sex Transm Infect. 2013;89: 25-27. 
45. van Bergen JE, Kerssens JJ, Schellevis FG, Sandfort TG, Coenen TT, Bindels PJ. Sexually transmitted infection health-care seeking behaviour in the Netherlands: general practitioner attends to the majority of sexually transmitted infection consultations. Int J STD AIDS. 2007; 18: 374-379.

46. Dukers-Muijrers NH, Niekamp AM, Vergoossen MM, Hoebe CJ. Effectiveness of an opting-out strategy for HIV testing: evaluation of 4 years of standard HIV testing in a STI clinic. Sex Transm Infect. 2009;85: 226-230.

47. Roekevisch E, de Vries HJC. Serie soa: proctitis. SOA AIDS MAG. 2010;1: 7-9.

48. LaMontagne DS, Fenton KA, Randall S, Anderson S, Carter P. Establishing the National Chlamydia Screening Programme in England: results from the first full year of screening. Sex Transm Infect. 2004;80: 335-341.

49. Turner K, Adams E, Grant A, Macleod J, Bell $G$, Clarke J, et al. Costs and cost effectiveness of different strategies for chlamydia screening and partner notification: an economic and mathematical modelling study.

BMJ. 2011;342: c7250.

50. CDC Fact Sheet: Reported STDs in the United States 2012 National Data for Chlamydia, Gonorrhea, and Syphilis

51. Turner KM, Adams EJ, Lamontagne DS, Emmett L, Baster K, Edmunds WJ. Modelling the effectiveness of chlamydia screening in England. Sex Transm Infect. 2006;82: 496-502.

52. van Bergen JE, Fennema JS, van den Broek IV, Brouwers EE, de Feijter EM, Hoebe CJ, et al.
Rationale, design, and results of the first screening round of a comprehensive, registerbased, Chlamydia screening implementation programme in the Netherlands. BMC Infect Dis. 2010;10: 293.

53. van den Broek IV, Brouwers EE, Gotz HM, van Bergen JE, Op de Coul EL, Fennema JS, Koekenbier RH, Pars LL, van Ravesteijn SM, Hoebe CJ. Systematic selection of screening participants by risk score in a Chlamydia screening programme is feasible and effective. Sex Transm Infect. 2012;88: 205-211.

54. van den Broek IV, Hoebe CJ, van Bergen JE, Brouwers EE, de Feijter EM, Fennema JS, et al. Evaluation design of a systematic, selective, internet-based, Chlamydia screening implementation in the Netherlands, 2008-2010: implications of first results for the analysis. BMC Infect Dis. 2010;10: 89.

55. van den Broek IV, Verheij RA, van Dijk CE, Koedijk FD, van der Sande MA, van Bergen JE. Trends in sexually transmitted infections in the Netherlands, combining surveillance data from general practices and sexually transmitted infection centers. BMC Fam Pract. 2010;11: 39.

56. van Aar F KF, van den Broek IVF, Op de Coul ELM, Soetens LC, Woestenberg PJ, Heijne JCM, van Sighem AI, Nielen MMJ, van Benthem BHB. Sexually transmitted infections in the Netherlands in 2013. RIVM rapport 150002005. National Institute for Public Health and the Environment; available from URL: http://www.rivm.nl/bibliotheek/ rapporten/150002005.pdf. 



\section{Section test policy, test evaluation}





\section{Chapter 2}

\section{The added value of chlamydia screening between 2008-2010 in reaching young people in addition to chlamydia testing in regular care; an observational study}

Geneviève AFS van Liere, Nicole HTM Dukers-Muijrers, Jan EAM van Bergen, Hannelore M Götz, Frans S Stals and Christian JPA Hoebe

BMC Infectious Diseases 2014; 14:612 


\section{Abstract}

\section{Introduction}

Internet-based Chlamydia Screening Implementation (chlamydia screening programme) was introduced in the Netherlands in 2008-2010 to detect and treat asymptomatic infections and to limit ongoing transmission through annual testing and treatment of Chlamydia trachomatis in young people (16-29 years). This population-based screening may be less effective when addressing individuals who are already covered by regular care, instead of addressing a hidden key population without chlamydia testing experience in regular care. This study had two aims: (1) to assess the rate and determinants of newly reached (i.e. not previously tested in 2006-2010) participants in the chlamydia screening programme, and (2) to assess the chlamydia positivity in these newly reached participants.

\section{Methods}

This observational matching study included all chlamydia tests performed in subjects aged 16-29 years in eastern South Limburg in the Netherlands (population 16-29 years: 41000) between 2006-2010. Testing was conducted during the systematic chlamydia screening programme (2008-2010), at a sexually transmitted infections clinic (STI clinic), by general practitioners (GPs), and by medical specialists as reported by the medical laboratory serving the region. Data were matched between testing services on individual level. The study population included all participants who were tested at least once for chlamydia by the chlamydia screening programme. Participants were included at their first chlamydia screening participation.

\section{Results}

In the chlamydia screening programme, $80.7 \%$ (4298/5323) of participants were newly reached, others were previously tested by the STI clinic (5.7\%, n=304), GPs (6.2\%, n=328), medical specialists $(3.5 \%, n=187)$ or a combination of providers $(3.9 \%, n=206)$. Chlamydia prevalence was similar in newly reached participants $(4.8 \%, 204 / 4298)$ and participants previously tested $(4.5 \%, 46 / 1025, \mathrm{P}=0.82)$. Independent determinants for being a newly reached participant were male gender (men $\mathrm{OR} 2.9 ; 95 \% \mathrm{Cl} 2.5-3.4$ ) and young age $<21$ years (versus $25-29$ years OR 1.8; $95 \% \mathrm{Cl} 1.5-2.2$ ).

\section{Discussion}

The majority of the chlamydia screening programme participants have not been tested by regular care, and show similar chlamydia prevalence as those previously tested. Thereby population-based chlamydia screening adds to the existing regular care by testing young individuals hidden to current regular care. 


\section{Introduction}

Chlamydia is the most prevalent treatable sexually transmitted infection worldwide and has major public health consequences, especially in young women [1]. Early detection and treatment is warranted to limit the spread of infection and to reduce sequelae in infected individuals. A possible complication is pelvic inflammatory disease, where Chlamydia trachomatis ascends to the upper genital tract causing tubal factor infertility and ectopic pregnancy. In the Netherlands the regular care for testing and treatment of chlamydia is provided by general practitioners (GPS), sexually transmitted infections (STI) clinics and after referral by medical specialists (mainly gynaecologists) [2]. Thus regular care providers are GP's, STI clinics and medical specialists. Systematic population-based internet chlamydia screening was initiated in 2008 and aimed to improve case finding to prevent sequelae and to reduce population prevalence by annual testing and treatment of people aged 16-29 years in three regions in the Netherlands. The choice for targeting 16 to 29 -year-olds in the additional chlamydia screening programme was based on the highest burden of chlamydia infection among these young people $[3,4]$. After a postal invitation, home sampling kits for urogenital testing (urine or vaginal swab) could be requested through a website (www.chlamydiatest.nl). Treatment and partner notification were done by the GP or at a STI clinic [5]. The rationale for the chosen approach in the chlamydia screening programme were based on existing evidence for screening programmes, costs, flexible communication, easy adaptation of the screening in time and the possibility of easy expansion to other geographic areas in the future[6]. Moreover, acceptability of the screening method using internet was high [7].

Keystones for an effective large-scale screening programme are achieving adequate levels of participation [8] and capturing substantial numbers of new (chlamydia positive) participants in addition to regular care like STI clinics and general practitioners (GPs). To understand and interpret the outcome of chlamydia screening, knowing who takes part in the chlamydia screening programme is essential [9]. By assessing the totality of chlamydia testing practices, it becomes clear whether the chlamydia screening programme reached persons already served by regular care or a hidden key population without chlamydia testing experience in regular care. Chlamydia screening would become less effective when reaching those who were already tested by regular care. Therefore chlamydia screening should target this hidden key population to prevent chlamydia sequelae in individuals and to diminish further spread of chlamydia in the population additional to the efforts in regular care. Publications assessing the totality of chlamydia testing practices including additional chlamydia screening in a programme are limited $[10,11]$, as such an assessment is frequently hampered by unavailability of data or unmatchable data sources. Therefore the rationale of this study was to bridge this gap and evaluate this second keystone by using a near complete large data collection of matched test data sources in the target region 
for the chlamydia screening programme. This study had two aims: (1) to assess the rate and determinants of newly reached men and women aged 16-29 years in the chlamydia screening programme in eastern South Limburg, and (2) to assess the chlamydia positivity in these newly reached men and women.

\section{Methods}

\section{Systematic chlamydia screening in the Netherlands}

The Dutch chlamydia screening programme used systematic-population-based internet chlamydia screening in three regions of the Netherlands, including the eastern South Limburg study area. The intervention was implemented by means of a stepped wedge design, with sequential roll out to geographical clusters of potential participants in a randomly determined order over time so that, by the end of the three year study period, each cluster had been invited at least once. The stepped wedge design was chosen to be able to evaluate participation and effectiveness over several rounds of screening. In three screening rounds from 2008-2010, all men and women aged 16-29 years who were listed in the study area's municipal population register ( $n=41,000,2010)$ were sent an invitation letter [5]. In our study area South Limburg, eligibility for chlamydia testing within the chlamydia screening programme depended on an individual's chlamydia risk score. This risk score was based on answers to an eight-item risk questionnaire (i.e. age, place of residence, education level, condom use at last intercourse, number of lifetime sex contacts, ethnic background, having a new sexual partner in the last 6 months and symptoms) [12]. When a person was eligible, home sampling kits for urogenital testing could be requested through a website (www.chlamydiatest.nl). Chlamydia screening participants could provide additional data via an optional electronic general questionnaire (hereafter questionnaire).

\section{Study design; data collection and matching}

Three data sources were used for all the men and women aged 16-29 years who were tested for chlamydia in the study area between 2006 and 2010: the chlamydia screening programme, an STI clinic, and the medical laboratory [13]. Data from GPs and medical specialists (mainly gynaecologists) were obtained from the regional medical microbiology laboratory covering the study area $(>95 \%)$. Data from the STI clinic were retrieved from our public health STI clinic's medical records comprising confirmed test results. The basis for data matching was the municipal population register, which included men and women aged 16-29 years who were invited for and tested in the chlamydia screening programme. Data from GPs and medical specialists were uniquely matched to the register on personal level by part of the last name, month of birth, year of birth, sex, and postal code. Part of the test records had identical part of the last name, month of birth, year of birth, sex, and postal code. These records were considered to belong to the same 
individual, and were matched to the identical municipal population register record. For chlamydia screening participants, a ranking order was assigned to all matched records from GPs and medical specialists (medical laboratory) for sensitivity analyses. Data from the STI clinic were all matched uniquely to the register based on the whole name and date of birth (figure 1).

Figure 1: Flow diagram of the data matching and study procedures

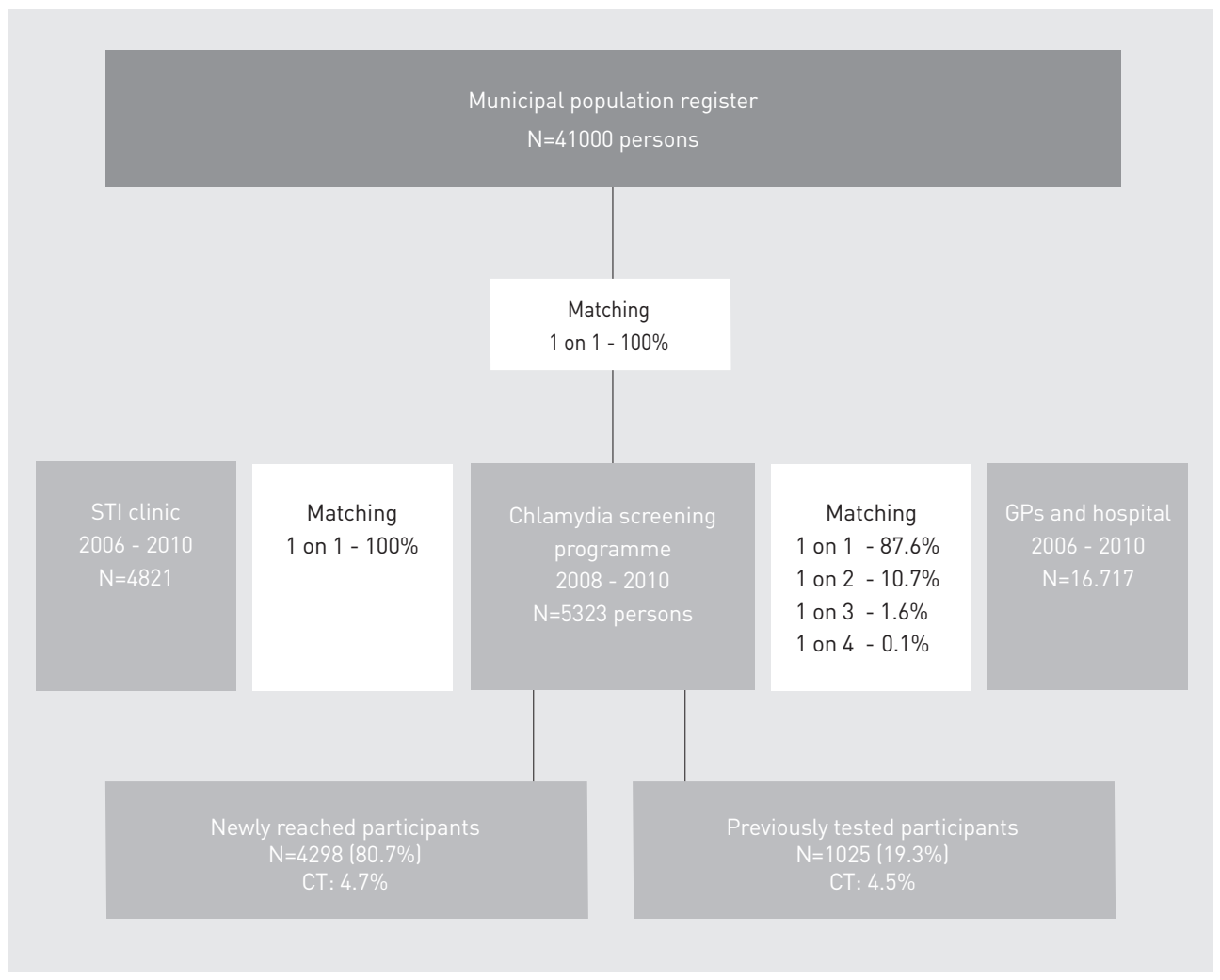

Data from the STI clinic, the chlamydia screening programme, GPS and the hospital were matched at individual level to the municipal population register. Data from the STI clinic and the chlamydia screening programme were matched uniquely. Data from GPs and hospital were matched by probabilistic algorithmic matching. The study population included all participants who were screened at least once for chlamydia ( $n=5395$ ). 


\section{Study population}

The study population included all participants who were tested at least once for chlamydia by the chlamydia screening programme. Participants were included their first chlamydia screening participation. Data on sex, age, and test result were available for all participants, data on same-sex behaviour, symptoms and number of sex partners in the past six months was only available for participants who filled in questionnaire. Variables used for analyses were sex, age $(\leq 21$, 22-24, 25-29 years (reference), based on tertiles), test result, nationality (Western vs. non-Western), same-sex behaviour (men who have sex with men, heterosexual men and women (reference)), symptoms, and number of sex partners in the past six months $(1,2, \geq 3$, based on tertiles).

\section{Chlamydia trachomatis diagnosis}

Specimens tested by the chlamydia screening programme and at the STI clinic came from mostly self-collected vaginal swabs and urine. GPs and medical specialists used mostly cliniciancollected urethral and cervical swabs. The STI clinic, GPs, and medical specialists used SDA and PCR for Chlamydia trachomatis testing (Becton Dickinson ProbeTec ET system, Maryland, USA and from 6-1-2010 Abbott M2000, Illinois, USA). The chlamydia screening programme used PCR (Roche Cobas Taqman, California, USA). All tests were performed according to the manufacturers' protocols.

\section{Analysis}

At the first chlamydia screening participation, we assessed whether participants were previously tested by one or more regular care providers between 2006-2010 based on matched data. Participants who were not previously tested were defined as 'newly reached participant'. The proportion of positive chlamydia tests was compared between newly reached participants and previously tested participants using a Chi-square test; adjusting for age and gender did not change the results. Logistic regression analysis was performed: being a newly reached participant was used as the outcome to assess the association with the determinants age, sex, nationality and test result. To assess the association between newly reached participant and determinants from the questionnaire, the second analysis was restricted to participants with a questionnaire. Assessed determinants included age, sex, nationality, sexual preference, symptoms, number of sex partners in the past six months and test result. To test for selection bias, age, sex, nationality, test result and newly reached participant were compared between participants with and without questionnaire using Chi square test. Independent determinants were assessed by multivariable analyses using stepwise backward selection. Interactions terms were added in the multivariable model but none were statistically significant and they were not included in the final model. All analyses were adjusted for year of invitation for the chlamydia screening. A p value $<0.05$ was considered to be statistically significant. Analyses were performed using the SPSS package version 20 (IBM Inc. Somers, New York, USA). 


\section{Ethics Statement}

Participants, including minors (16-18 years-old), provided written consent to participate in this study, including consent for further research. No written consent was obtained from next of kin, caretakers, or guardians on behalf of the minors enrolled in the study. The Medical Ethics Committee of the VU University Amsterdam (Identification number 2007/239) approved the chlamydia screening trial. The Medical Ethics Committee of Maastricht University IIdentification number 12-4-042) approved the study, including the consent procedure and data matching.

\section{Results}

\section{Data matching}

The STI clinic database comprised 4821 young people (16-29 years), the GP and hospital database comprised 16.717 young people. In total 41.000 young people were invited for chlamydia screening, 5395 participated at least once (13.2\%). Participants who were 16 years old at the first chlamydia screening ( $n=72$ ) were excluded from analysis because there were no previous testing data available from regular care providers. The study population comprised 5323 participants (participation 13.0\%). Of all test records matched from GPs and medical specialists with the chlamydia screening participants in the municipal population register $(n=1287), 87.6 \%$ ( $n=1127)$ were matched uniquely, $10.7 \%(n=138)$ were matched 1 on $2,1.6 \%(n=21)$ were matched 1 on 3 , and $0.1 \%(n=1)$ was matched 1 on 4 . Data from the STI clinic were all uniquely (1 on 1) matched based on first name, last name, date of birth, sex, and postal code ( $\mathrm{n}=422$ ). In total, $90.6 \%$ (1549/1709) of data were uniquely matched (1 on 1) (figure 1).

\section{Study population}

The largest proportion of participants consisted of participants 25-29 years of age, followed by participants of 22-24 years of age. Two thirds of participants were women, and the majority had Western nationality (>95\%). In total, $59.1 \%$ ( $n=3162$ ) of participants filled in the questionnaire. Participants without questionnaire were older, more often men, more often had non-Western nationality and had lower chlamydia prevalence compared to participants with questionnaire (table 1). 
Table 1: Characteristics of chlamydia screening programme participants without and with questionnaire

\begin{tabular}{|c|c|c|c|}
\hline \multirow[t]{3}{*}{ Variables } & $\begin{array}{l}\text { Participants } \\
\text { without questionnaire }\end{array}$ & $\begin{array}{l}\text { Participants } \\
\text { with questionnaire }\end{array}$ & Total \\
\hline & $\mathrm{N}=2161$ & $N=3162$ & $N=5323$ \\
\hline & $\%(n)$ & $\%(n)$ & $\%(n)$ \\
\hline \multicolumn{4}{|l|}{ Age } \\
\hline$\leq 21$ & $24.2(523)$ & $28.7(906)^{*}$ & $26.8(1429)$ \\
\hline $22-24$ & $36.1(781)$ & $34.2(1081)$ & $35.0(1862)$ \\
\hline $25-29$ & 39.7 (857) & $37.2(1175)$ & 38.2 (2032) \\
\hline \multicolumn{4}{|l|}{ Sex } \\
\hline Men & 42.5 (919) & 29.1 (919)* & 34.5 (1838) \\
\hline Women & 57.5 (1242) & $70.9(2243)$ & 65.5 (3485) \\
\hline \multicolumn{4}{|l|}{ Nationality } \\
\hline Non-Western & $3.8(81)$ & $2.0(64)^{*}$ & $2.7(145)$ \\
\hline \multicolumn{4}{|l|}{ Chlamydia } \\
\hline Yes & $2.9(63)$ & $5.9(181)^{*}$ & $4.7(250)$ \\
\hline \multicolumn{4}{|c|}{ Newly reached participant } \\
\hline Yes & $80.8(1746)$ & $80.7(2552)$ & 80.7 (4298) \\
\hline
\end{tabular}

* $P<0.01$

\section{Newly reached participants}

In the chlamydia screening programme, 80.7\% (4298/5323) of participants were newly reached (not previously tested). The other participants had been tested previously at least once: $5.7 \%$ (304/5323) by the STI clinic, $6.2 \%(328 / 5323)$ by GPs, 3.5\% (187/5323) by medical specialists, and $3.9 \%(206 / 5323)$ by a combination of providers. The proportion of newly reached participants was comparable in participants with and without questionnaire (table 1). Of all previous tests, $2.7 \%$ ( $n=28$ ) were tested within 3 months before chlamydia screening participation.

\section{Determinants newly reached participants}

In multivariable analyses, independent determinants for a newly reached participant were male gender and young age. Nationality and test result were not associated. In restricted analyses including questionnaire data, further identified independent determinants for newly reached participants were young age, having had 1 or 2 sex partners (compared to 3 sex partners), being a man who had sex with men (MSM), and being a heterosexual man (compared to women). Sex, symptoms, or test result (see section below) were not associated (table 2). 
Table 2: Characteristics of newly reached participants and previously tested participants

\begin{tabular}{|c|c|c|c|c|c|}
\hline Variables & $\begin{array}{l}\text { Newly reached } \\
\text { participants }\end{array}$ & $\begin{array}{l}\text { Previously } \\
\text { tested }\end{array}$ & $\begin{array}{l}\text { Unadjusted } \\
\text { OR }\end{array}$ & $\begin{array}{l}\text { Adjusted } \\
\text { OR }\end{array}$ & $P$ value \\
\hline & $N=4298$ & $N=1025$ & & & \\
\hline & $\%(n)$ & $\%(n)$ & $(95 \% \mathrm{CI})$ & $(95 \% \mathrm{Cl})$ & \\
\hline \multicolumn{6}{|l|}{ Age } \\
\hline$\leq 21$ & 28.7 (1233) & 19.1 (196) & $1.63(1.4-2.0)$ & $1.8(1.5-2.2)$ & $<0.001$ \\
\hline $22-24$ & $33.8(1451)$ & $40.1(411)$ & $0.9(0.8-1.1)$ & $1.0(0.8-1.1)$ & 0.56 \\
\hline $25-29$ & $37.6(1614)$ & $40.8(418)$ & 1 & 1 & \\
\hline \multicolumn{6}{|l|}{ Sex } \\
\hline Men & $38.3(1646)$ & 18.7 (192) & $2.7(2.3-3.2)$ & $2.9(2.5-3.4)$ & $<0.001$ \\
\hline Women & 61.7 (2652) & 81.3 (833) & 1 & 1 & \\
\hline \multicolumn{6}{|l|}{ Chlamydia } \\
\hline Yes & $4.8(204)$ & $4.5(46)$ & 1.1 (0.8-1.5) & ns & \\
\hline No & & & 1 & & \\
\hline \multicolumn{6}{|l|}{ Nationality } \\
\hline Non-Western & $2.6(111)$ & $3.3(34)$ & $0.8(0.5-1.2)$ & & \\
\hline Western & 96.7 (990) & 97.4 (4159) & 1 & ns & \\
\hline \multirow[t]{3}{*}{$\begin{array}{l}\text { Ristricted } \\
\text { Aanalysis }\end{array}$} & $\begin{array}{l}\text { Newly reached } \\
\text { participants }\end{array}$ & $\begin{array}{l}\text { Previously } \\
\text { tested }\end{array}$ & $\begin{array}{l}\text { Unadjusted } \\
\text { OR }\end{array}$ & $\begin{array}{l}\text { Adjusted } \\
\text { OR }\end{array}$ & $P$ value \\
\hline & $N=2552$ & $\mathrm{~N}=610$ & & & \\
\hline & $\%(n)$ & $\%(n)$ & $(95 \% \mathrm{Cl})$ & $(95 \% \mathrm{Cl})$ & \\
\hline \multicolumn{6}{|l|}{ Age } \\
\hline$\leq 21$ & 30.7 (783) & 20.2 (123) & $1.7(1.4-2.2)$ & $1.7(1.3-2.2)$ & $<0.001$ \\
\hline $22-24$ & 33.0 (842) & 39.2 (239) & $0.9(0.8-1.2)$ & $1.0(0.8-1.2)$ & 0.78 \\
\hline $25-29$ & 36.3 (927) & 40.7 (248) & 1 & 1 & 1 \\
\hline \multicolumn{6}{|l|}{ Sex } \\
\hline Men & 32.4 (827) & 15.1 (92) & $2.7(2.1-3.4)$ & ns & \\
\hline Women & 67.6 (1725) & 84.9 (518) & 1 & & \\
\hline \multicolumn{6}{|l|}{ Chlamydia } \\
\hline Yes & $5.9(150)$ & 6.1 (37) & $1.0(0.7-1.4)$ & ns & \\
\hline No & 94.1 (2402) & 93.9 (573) & 1 & & \\
\hline
\end{tabular}




\begin{tabular}{|c|c|c|c|c|c|}
\hline \multirow[t]{3}{*}{$\begin{array}{l}\text { Restricted } \\
\text { analyses }\end{array}$} & $\begin{array}{l}\text { Newly reached } \\
\text { participants }\end{array}$ & $\begin{array}{l}\text { Previously } \\
\text { tested }\end{array}$ & $\begin{array}{l}\text { Unadjusted } \\
\text { OR }\end{array}$ & $\begin{array}{l}\text { Adjusted } \\
\text { OR }\end{array}$ & $P$ value \\
\hline & $\mathrm{N}=2552$ & $\mathrm{~N}=610$ & & & \\
\hline & $\%(n)$ & $\%(n)$ & $(95 \% \mathrm{CI})$ & $(95 \% \mathrm{Cl})$ & \\
\hline \multicolumn{6}{|l|}{ Sexual preferences } \\
\hline Men who have sex with men & 1.7 (39) & $0.9(5)$ & $2.3(0.9-5.9)$ & $4.0(1.6-10.6)$ & $<0.01$ \\
\hline Heterosexual men & 30.4 (715) & $13.8(77)$ & $2.8(2.2-3.6)$ & $3.3(2.6-4.3)$ & $<0.001$ \\
\hline Women & $67.9(1598)$ & $85.3(477)$ & 1 & 1 & \\
\hline \multicolumn{6}{|l|}{ Sex partners } \\
\hline 1 & $73.3(1713)$ & $63.8(353)$ & $1.8(1.4-2.4)$ & $2.6(1.9-3.4)$ & $<0.001$ \\
\hline 2 & $15.9(371)$ & $19.2(106)$ & $1.3(0.9-1.8)$ & $1.7(1.2-2.4)$ & $<0.01$ \\
\hline$\geq 3$ & 10.8 (253) & $17.0(94)$ & 1 & 1 & \\
\hline \multicolumn{6}{|l|}{ Symptoms } \\
\hline Yes & $2.4(62)$ & $3.0(18)$ & $1.3(0.8-2.1)$ & ns & \\
\hline No & $97.6(2490)$ & $97.0(592)$ & 1 & & \\
\hline
\end{tabular}

Crude percentages and independent determinants for being a newly reached participant in univariate and multivariable analyses. Analyses were corrected for age, sex, and year of invitation. Restricted analyses include questionnaire data and were corrected for age, sexual preference, number of sex partners, and year of invitation.

\section{Discussion}

This study assessed the totality of chlamydia tests in one geographic region by matching all chlamydia testing data to the municipal population register, including testing by systematic population-based chlamydia screening, the STI clinic, GPs and medical specialists.

The chlamydia screening programme predominantly addressed new, previously untested, young participants who were not reached by regular care $(81 \%)$ and who had similar urogenital chlamydia prevalence as participants previously tested elsewhere ( $4.8 \%$ vs. $4.5 \%$ ). Therefore, we can conclude that the chlamydia screening programme adds to the existing regular care by revealing a so-far hidden population.

In this study, all chlamydia tests were collected: by regular care (GPS, STI clinic and medical specialists) and by intervention (chlamydia screening). There was no specific promotion of chlamydia testing during the chlamydia screening period that could have biased our results [5]. Previous studies also matched data from sexually transmitted infections/medical conditions diagnosed in several care settings to assess the proportion positives [11], births [14], ectopic 
pregnancies [14, 15], and reproductive capacity [15] after a chlamydia test. Our study adds to these previous studies by using the municipal population register which covers the entire population young people in one geographic region. This enabled assessing the totality of chlamydia testing, which was previously unknown [10].

Previous studies analysed participants in the English National Chlamydia Screening Programme (NCSP) and part of the Dutch systematic chlamydia screening programme. The NCSP is an opportunistic screening programme that offers chlamydia screening to eligible, sexually active individuals younger than 25 years when they attend consultations at specified health care, or other, settings [16]. Socio-demographic factors associated with participation in England were female gender and younger age [17]. Moreover, coverage was higher in deprived populations [18]. In the urbanised regions in the Netherlands, excluding this South Limburg study area, these factors were female gender and older age [8]. Separate analyses of South Limburg data gave similar results: female gender and older age were associated with participation (data not shown). In this study, newly reached participants turned out to be more often men, young $(<21$ years), and had had fewer sex partners compared to participants previously tested by regular care. This is important since men are usually harder to reach in screening programmes [16]. A possible explanation for this could be that men prefer testing in non-clinical settings such as postal testing kits and internet based screening [19]. In England only 15\% of young men were tested for chlamydia last year in contrast to $35 \%$ of young women [20]. A qualitative study examining the barriers and facilitators of offering chlamydia testing in general practitioners (GPs) and practice nurses revealed that women have more consultations and it is easier to raise sexual health issues within the type of consultations women are seeking. Moreover, awkwardness and embarrassment were reported in raising chlamydia screening with men [21]. Another study found that GPs are reluctant to test young people for chlamydia in absence of urogenital symptoms [22]. Altogether, internet based screening could be helpful to stimulate especially young men [23]. New e-health strategies could provide more insight in the sexual and testing behaviour of young people [24].

Younger age is known to be associated with testing positive for chlamydia $[8,20]$, this makes young people a target group to reach in chlamydia screening. New participants had fewer sex partners, an explanation could be that people with more sex partners are more likely to attend STI clinics [25].

The chlamydia prevalence of newly reached participants was comparable to that of participants previously tested by regular care, and groups tested in other healthcare settings such as the STD clinic [26] and GPs [27]. This indicates that the chlamydia screening programme complements regular care in detecting chlamydia positives. 
Sex was associated with being a newly reached participant in overall analyses, but not in additional analyses on questionnaire data. A possible explanation could be that a smaller proportion of men filled in the questionnaire compared to women. Being MSM was associated with being a newly reached participant, although this key population needs additional extra genital testing to fully address their risk behaviour [26]. Chlamydia testing was limited to urogenital site, which might underestimate the true burden of chlamydia as other studies showed that prevalence of anorectal chlamydia is substantial in both MSM and women $[26,28,29]$.

This study has several limitations. First, it consists of not $100 \%$ uniquely matched data but $91 \%$. The last $9 \%$ was matched using test records with identical markers from GPs and medical specialists. This slight inaccuracy might have introduced some bias and could lead to an underestimation of the proportion new participants. However, we expect this bias to be negligible as a sensitivity analysis on uniquely matched data revealed similar results. Unfortunately, data from care providers were only available for a restricted timeframe (2006-2010), limiting our results. It is possible that newly reached participants could have visited regular care before 2006 .

Another limitation is that additional data on sexual behaviour was only available for the group that filled in extra self-administered questionnaires, which could lead to bias for the restricted data analysis. However, odds ratios for age were similar for the main analysis and the restricted data analysis (see table 1), indicating that bias was unlikely.

The study area itself was another limitation. The eastern South Limburg region in the Netherlands is the first study area with sufficient and matchable data sources that can be used to assess the totality of chlamydia testing. However, this region is partly rural and it is unknown where data can be extrapolated to other participation regions that are more urban, such as Amsterdam and Rotterdam. We have to acknowledge that the generalizability of our results might be confined as screening programmes in different countries vary widely because of differences in recruitment methods, sample frames and participation rates. Moreover, not all persons in eastern South Limburg requesting chlamydia testing through the website were eligible due to the individuals' chlamydia risk score. This may have led to higher positivity rates compared to systematic screening [12]. In a postal non-response study, no indications were found that participation in the screening was hampered by limited access to the internet. Non response to the screening was largely based on perceptions of individual risk [6]. 


\section{Conclusions}

This study contributes to understanding the impact of chlamydia screening in reaching previously untested young people. In this study, chlamydia screening reached a hidden key population of young men and women who had never been tested before and who as a group showed a prevalence of chlamydia comparable to clinical settings.

\section{Acknowledgements}

The authors thank the staff of the STI Clinic South Limburg, the Department of Medical Microbiology, Atrium Medical Centre Parkstad and the Dutch Chlamydia Screening Implementation (CSI) project group especially IVF van den Broek, EEHG Brouwers, JSA Fennema, RH Koekenbier, ELM Op de Coul, LL Pars and SM van Ravesteijn for their valuable contribution to the CSI data collection. 


\section{References}

1. Workowski KA, Berman S: Sexually transmitted diseases treatment guidelines, 2010. MMWR Recomm Rep 59(RR-12):1-110.

2. van den Broek IV, Verheij RA, van Dijk CE, Koedijk FD, van der Sande MA, van Bergen JE: Trends in sexually trans-mitted infections in the Netherlands, combining surveillance data from general practices and sexually trans-mitted infection centers. BMC Fam Pract 2010, 11:39.

3. CDC Fact Sheet: Reported STDs in the United States 2012 National Data for Chlamydia, Gonorrhea, and Syphilis [http://www.cdc.gov/ nchhstp/newsroom/docs/STD-Trends-508.pdf ]

4. Kalwij S, Macintosh M, Baraitser P: Screening and treatment of Chlamydia trachomatis infections. BMJ 2010, 340:c1915.

5. van den Broek IV, van Bergen JE, Brouwers EE, Fennema JS, Gotz HM, Hoebe CJ, Koekenbier RH, Kretzschmar M, Over EA, Schmid BV, Pars LL, van Ravesteijn SM, van der Sande MA, de Wit GA, Low N, Op de Coul EL: Effectiveness of yearly, register based screening for chlamydia in the Netherlands: controlled trial with randomised stepped wedge implementation. BMJ 2012, 345:e4316.

6. van Bergen JE, Fennema JS, van den Broek IV, Brouwers EE, de Feijter EM, Hoebe CJ, Koekenbier RH, de Coul EL, van Ravesteijn SM, Gotz HM: Rationale, design, and results of the first screening round of a comprehensive, register-based, Chlamydia screening implementation programme in the Netherlands. BMC Infect Dis 2010, 10:293.

7. Greenland KE, Op de Coul EL, van Bergen JE, Brouwers EE, Fennema HJ, Gotz HM, Hoebe
CJ, Koekenbier RH, Pars LL, van Ravesteijn SM, van den Broek IV: Acceptability of the internet-based Chlamydia screening implementation in the Netherlands and insights into nonresponse. Sex Transm Dis 2011, 38(6):467-474.

8. Op de Coul EL, Gotz HM, van Bergen JE, Fennema JS, Hoebe CJ, Koekenbier RH, Pars LL, van Ravesteijn SM, van der Sande MA, van den Broek IV: Who participates in the Dutch Chlamydia screening? A study on demographic and behavioral correlates of participation and positivity. Sex Transm Dis 2012, 39(2):97-103.

9. Heijne JC, Low N: Differential selection processes in opportunistic chlamydia screening. Sex Transm Infect 2011, 87(6):454-455.

10. Bone A, Soldan K, Woodhall S, Clarke J, Gill ON: Opportunistic or population register based programmes for chlamydia screening? BMJ 2012, 345:e5887.

11. Slater W, Sadler K, Cassell JA, Horner P, Low $\mathrm{N}$ : What can be gained from comprehensive disaggregate surveillance? The Avon Surveillance System for Sexually Transmitted Infections. Sex Transm Infect 2007, 83(5):411-415.

12. van den Broek IV, Brouwers EE, Gotz HM, van Bergen JE, Op de Coul EL, Fennema JS, Koekenbier RH, Pars LL, van Ravesteijn SM, Hoebe CJ: Systematic selection of screening participants by risk score in a Chlamydia screening programme is feasible and effective. Sex Transm Infect 2012, 88(3):205-211.

13. Dukers-Muijrers NH, van Liere GA, Hoebe CJ: Re-screening Chlamydia trachomatis positive subjects:a comparison of practices between 
an STI clinic, general practitioners and gynaecologists. Sex Transm Infect 2013, 89(1):25-27.

14. Bakken IJ, Skjeldestad FE, Lydersen S, Nordbo SA: Births and ectopic pregnancies in a large cohort of women tested for Chlamydia trachomatis. Sex Transm Dis 2007, 34(10):739-743.

15. Andersen B, Ostergaard L, Puho E, Skriver MV, Schonheyder HC: Ectopic pregnancies and reproductive capacity after Chlamydia trachomatis positive and negative test results: a historical follow-up study. Sex Transm Dis 2005, 32(6):377-381.

16. Low N, Bender N, Nartey L, Shang A, Stephenson JM: Effectiveness of chlamydia screening: systematic review. Int J Epidemiol 2009, 38(2):435-448.

17. Riha J, Mercer CH, Soldan K, French CE, Macintosh $\mathrm{M}$ : Who is being tested by the English National Chlamydia Screening Programme? A comparison with national probability survey data. Sex Transm Infect 2011, 87(4):306-311.

18. Sheringham J, Simms I, Riha J, Talebi A, Emmett L, Macintosh M, Raine R: Will chlamydia screening reach young people in deprived areas in England? Baseline analysis of the English National Chlamydia Screening Programme delivery in 2008. Sex Transm Dis 2011, 38(8):677-684.

19. Lorimer K, Reid ME, Hart GJ: Willingness of young men and women to be tested for Chlamydia trachomatis in three non-medical settings in Glasgow, UK. J Fam Plann Reprod Health Care 2009, 35(1):21-26.
20. Wise J: Only $15 \%$ of young men in England were tested for chlamydia last year despite recommendations. BMJ 2014, 348:g4121.

21. Lorimer K, Martin S, McDaid LM: The views of general practitioners and practice nurses towards the barriers and facilitators of proactive, internet-based chlamydia screening for reaching young heterosexual men. BMC Fam Pract 2014, 15:127.

22. McNulty CA, Freeman E, Bowen J, Shefras J, Fenton KA: Diagnosis of genital chlamydia in primary care: an explanation of reasons for variation in chlamydia testing. Sex Transm Infect 2004, 80(3):207-211.

23. Lorimer K, McDaid L: Young men's views toward the barriers and facilitators of Internet-based Chlamydia trachomatis screening: qualitative study. J Med Internet Res 2013, 15(12):e265.

24. Theunissen KA, Hoebe CJ, Crutzen R, Kara-Zaitri C, de Vries NK, van Bergen JE, van der Sande MA, Dukers-Muijrers NH: Using intervention mapping for the development of a targeted secure web-based outreach strategy named SafeFriend, for Chlamydia trachomatis testing in young people at risk. BMC Public Health 2013, 13(1):996.

25. de Coul EL, Warning TD, Koedijk FD: Sexual behaviour and sexually transmitted infections in sexually transmitted infection clinic attendees in the Netherlands, 2007-2011. Int J STD AIDS 2014, 25(1):40-51.

26. van Liere GA, Hoebe CJ, Niekamp AM, Koedijk FD, Dukers-Muijrers NH: Standard symptomand sexual history-based testing misses 
anorectal Chlamydia trachomatis and neisseria gonorrhoeae infections in swingers and men who have sex with men.

Sex Transm Dis 2013, 40(4):285-289.

27. van Bergen JE, Kerssens JJ, Schellevis FG, Sandfort TG, Coenen TT, Bindels PJ: Sexually transmitted infection health-care seeking behaviour in the Netherlands: general practitioner attends to the majority of sexually transmitted infection consultations. Int J STD AIDS 2007, 18(6):374-379.

28. van Liere GA, Hoebe CJ, Dukers-Muijrers NH: Evaluation of the anatomical site distribution of chlamydia and gonorrhoea in men who have sex with men and in high-risk women by routine testing: cross-sectional study revealing missed opportunities for treatment strategies. Sex Transm Infect 2014, 90(1):58-60.

29. van Liere GA, Hoebe CJ, Wolffs PF, DukersMuijrers NH: High co-occurrence of anorectal chlamydia with urogenital chlamydia in women visiting an STI clinic revealed by routine universal testing in an observational study; a recommendation towards a better anorectal chlamydia control in women. BMC Infect Dis 2014, 14(1):274. 



\section{Chapter 3}

Who tests whom? A comprehensive overview of Chlamydia trachomatis test practices in a Dutch region among different STI care providers for urogenital, anorectal and oropharyngeal sites in young people: a cross-sectional study

Casper DJ den Heijer, Geneviève AFS van Liere, Christian JPA Hoebe, Jan EAM van Bergen, Jochen WL Cals, Frans S Stals, Nicole HTM Dukers-Muijrers

Sexual Transmitted Infections 2015; aug 11 doi:

10.1136/sextrans-2015-052065 


\section{Abstract}

\section{Introduction}

To evaluate and compare Chlamydia trachomatis (CT) diagnostic test practices of different sexually transmitted infection (STI) care providers in 16-29 year-olds from one defined geographic Dutch region (280000 inhabitants). Both number and proportion of positive CT tests (test positivity) were assessed, and factors associated with these outcomes.

\section{Methods}

Data on laboratory testing and diagnosis of urogenital, anorectal and oropharyngeal CT between 2006 and 2010 were retrieved from general practitioners (GPs), gynaecologists, an STI clinic and a population-based chlamydia screening programme. Multivariable regression analyses explored associations between age, sex, test year, socio-economic status (SES), and STI care provider and the outcomes being the number of tests and test positivity.

\section{Results}

Overall, 22831 tests were performed (1868 positive; 8.2\%). Extra-genital (anorectal and oropharyngeal) tests accounted for $4 \%$ of all tests ( $7.5 \%$ positive) and were almost exclusively (99\%) performed by the STI clinic. STI clinics tested most men (37.2\% of all tested men), whereas GPs tested most women (29.9\% of all tested women). GPs and STI clinics accounted for $73.3 \%(1326 / 1808)$ of urogenital CT diagnoses.

In women, the number of tests increased with age, whereas test positivity decreased for all STI care providers. Lower SES was associated with higher test positivity in GP and gynaecology patients.

\section{Discussion}

STI clinics performed most CT tests in men, whereas GPs performed most CT tests in women. GPs and STI clinics accounted for the majority of positives. Extra-genital CT testing is rarely performed outside the STI clinic and needs to be promoted, especially in men who have sex with men. 


\section{Introduction}

Chlamydia trachomatis (CT) is the most common bacterial sexually transmitted infection (STI) in Europe. The proportion of positive CT tests is generally $5-10 \%$ in sexually active young people[1]. The high proportion of asymptomatic episodes, result in many undiagnosed CT infections[1]. If left untreated, CT can lead to several adverse sequelae, such as pelvic inflammatory disease (PID), and infertility[2].

Dutch STI care is organised in a similar way as in the UK [3] and Australia,[4] with a major role for public health care, that is, STI clinics, and general practitioners (GPS). These STI clinics serve specific high-risk groups, including young people laged <25). In hospitals, CT tests are predominantly performed by gynaecologists as part of a diagnostic work-up in fertility counselling, before inserting intra-uterine devices, or as part of the diagnostic evaluation of suspected PID. In addition to regular STI care as described above, CT screening programmes have been established, such as in the UK and the Netherlands[5,6]. Each of these STI care providers or programmes will contribute to CT control, yet a comprehensive assessment of their relative contribution in terms of testing, diagnosis, and the populations that they serve, is thus far unavailable. Sentinel data from Dutch GPs suggests that GPs diagnose the lion's share of CT cases, with estimates reaching $70 \%$ (versus $30 \%$ in STI clinics)[7]. Data from the National Chlamydia Screening Programme lyear 2013) in the UK (15-24 year-olds), show that the contribution of GPs seems smaller, accounting for $14 \%$ of all positive tests (versus $>40 \%$ by genitourinary (GUM) clinics)[8]. Recently, an extensive evaluation of Australian CT surveillance was performed, although not providing the relative contribution of the different STI care providers[9]. Moreover, the contribution of other STI care providers (eg, gynaecologists) has not yet been explored.

The determinants age[9], sex[9] and socio-economic status (SES)[10] have all been associated with CT test practice, that is, the number of tests performed and the proportion of positive tests, although it is unknown whether these associations differ between Dutch STI care providers.

While CT control strategies focus on urogenital CT testing, men who have sex with men (MSM) guidelines recommend that extra-genital testing is also performed[11]. In addition, the prevalence of anorectal CT in women attending STI clinics has been shown to be similar to that in MSM lup to $15 \%$ in women[12,13] vs $14 \%$ in MSM)[14]. Moreover, $>80 \%$ of all anorectal infections in MSM, and $20 \%$ in women attending STI clinics, are isolated and, thus, will be missed by urogenital testing alone[15]. Prevalence of oropharyngeal CT is lower: $1 \%$ in women[13,16] and 3\% in MSM[14]. These figures illustrate that extra-genital CT could contribute to CT transmission and CT-related morbidity[13,16]. 
Here, we aimed to provide a comprehensive description of Dutch CT urogenital and extra-genital test practices of the following STI care providers: GPs, an STI clinic, gynaecologists and a population-based chlamydia screening programme (here onwards referred to as 'chlamydia screening'). We examined data from 16-29 year olds from one defined geographic Dutch region from 2006 to 2010 . We assessed the number of CT tests and the proportion of positive tests (here onwards referred to as 'test positivity') across providers. Hence, this shows the relative contribution of different providers regarding urogenital and extra-genital CT control. We also examined the type of populations served regarding age, sex and SES.

\section{Methods}

\section{Study population}

We used three data sources to obtain all CT tests from January 2006 to August 2010: STI clinic ( $n=28591$ ), chlamydia screening ( $n=6489)$, and the medical laboratory serving both GPs and hospital physicians ( $n=24902$ ). All data sources covered a nearly complete $(>95 \%$ ) region in the southern part of the Netherlands (Parkstad, eastern South Limburg). From 2006 to 2010, Parkstad had a population of 41000 16-29 year-olds (total population: 280000; http://www.cbs.nl). STI clinic data were retrieved from our own public health STI clinic medical records. Data from the municipal population register included persons aged 16-29 years who had been tested during chlamydia screening. This programme used systematic-population-based internet chlamydia screening with a randomised stepped wedge approach. It consisted of three screening rounds (from 2008 to 2010) among 16-29 year-olds in three Dutch regions, including Parkstad. Details of chlamydia screening have been described elsewhere[5,17]. Eligibility for chlamydia testing in Parkstad was dependent on an individual's chlamydia risk score, calculated using an eight-item questionnaire lassessing age, place of residence, education level, condom use at last intercourse, number of lifetime sex contacts, ethnic background, having a new sexual partner in the last six months, and symptoms)[18].

All data sources provided age, sex, and four-digit postal code of the tested person, as well as date, anatomic location and result of the test. Using postal code and age, records were selected of patients aged 16-29 years living in Parkstad. Hospital physicians included 14 different specialties of which gynaecology was predominant (90.9\%). In order to reduce heterogeneity between hospital physicians, tests by non-gynaecology medical specialties, that is, 458 urogenital CT tests, were excluded from analyses. This resulted in 22831 tests for analyses: STI clinic ( $n=5475$ ), chlamydia screening ( $n=6427$ ), and GPs and gynaecologists ( $n=10929$ ) (figure 1).

Dutch SES scores were extracted per postal code area and are based on national data on income, educational level and employment (http://www.scp.nl)[19]. 


\section{CT diagnosis}

Most specimens tested during chlamydia screening and at the STI clinic were self-collected vaginal swabs and urine. GPs and gynaecologists predominantly used clinician-collected urethral and cervical swabs. Anorectal (mainly self-collected) and oropharyngeal (mainly providercollected) swabs were used for testing these respective anatomical locations. The STI clinic, GPS and gynaecologists all used strand displacement amplification and PCR for CT testing (Becton Dickinson ProbeTec ET system, Maryland, USA, and from 6-1-2010 Abbott M2000, Illinois, USA). Chlamydia screening used PCR (Roche Cobas Taqman, California, USA). All tests were performed according to the manufacturer's protocols.

\section{Statistical analyses}

First, a descriptive analysis was performed to assess the contribution of each STI care provider regarding the number of tests performed and positives diagnosed.

Second, to assess factors associated with the number of CT tests performed and test positivity, multivariable Poisson and logistic regression analyses were performed, respectively, including provider (GP, STI clinic, gynaecologists and chlamydia screening), age (16-21, 22-24 and 25-29)[20], SES (low, middle and high, based on tertiles), and test calendar year (continuous). Denominator data for all subgroups included in Poisson regression analyses were retrieved from Statistics Netherlands (www.cbs.nl; age, sex and test year) and the Netherlands Institute for Social Research (www.scp.nl; SES).

The multivariable analysis was initially performed including 'provider' and potential confounders (age, test year and SES) to test for differences in outcomes between STI care providers. Subsequent analyses to determine associations between the outcomes and age, test year and SES were stratified by STI care provider, because of statistically significant interactions between 'provider' and the other factors.

All analyses were stratified by sex and anatomic location: urogenital (urine, cervical, vaginal and urethral) and extra-genital (anorectal and oropharyngeal). Outcome measures presented are adjusted for age, test year and SES (where appropriate).

Analyses were performed using SPSS V.20 (IBM SPSS Statistics for Windows, IBM Corp., Armonk, New York, USA). A p value $<0.05$ was considered statistically significant. 
Figure 1: Selection procedure for the records analysed per database. GP, general practitioner; STI, sexually transmitted infection

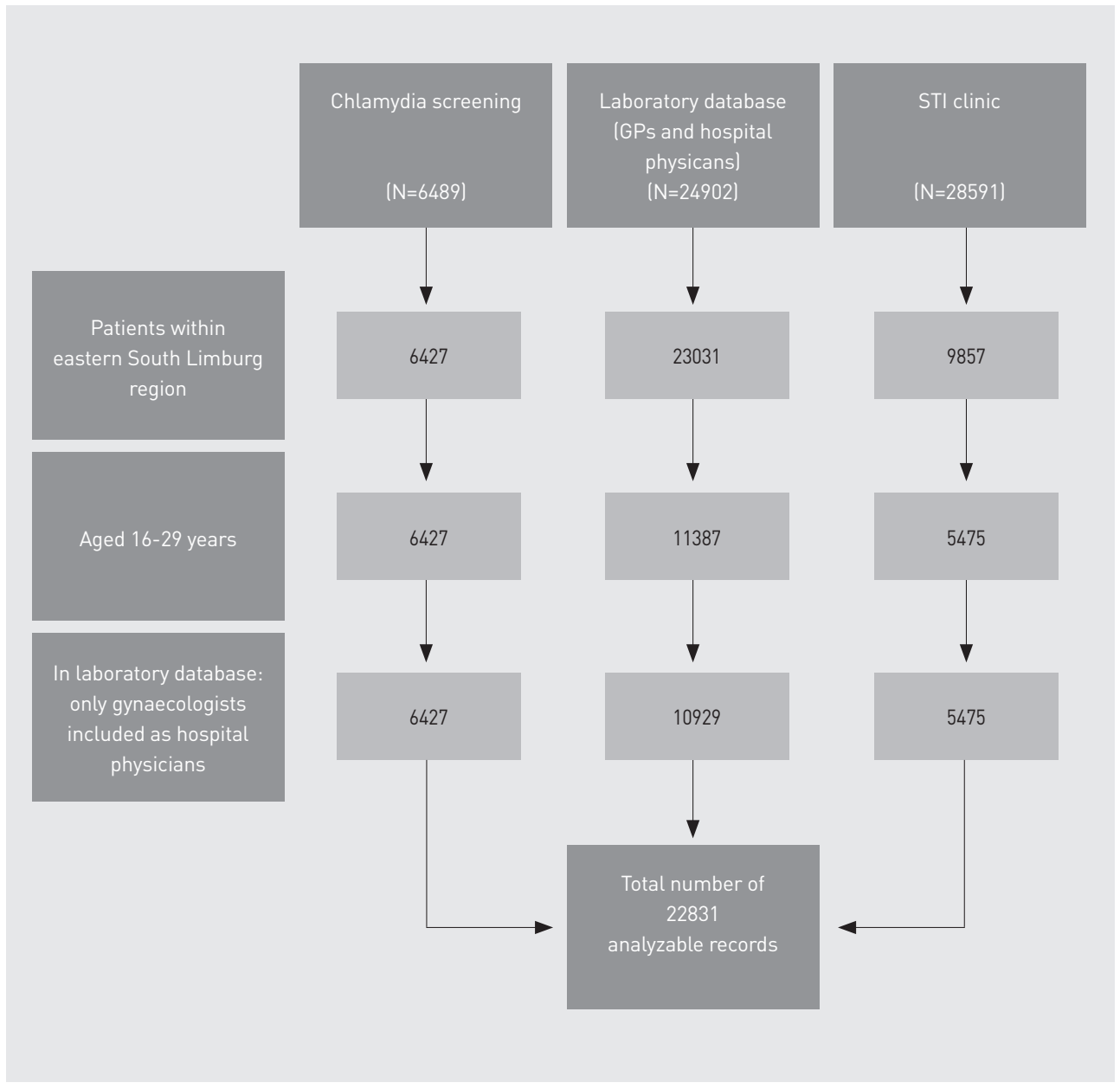

\section{Results}

In total, $22831 \mathrm{CT}$ tests were performed, including 1868 (8.2\%) positives. Stratified by anatomical location, test numbers (test positivity) were: urogenital 22029 (8.2\%), anorectal $556(10.1 \%)$ and oropharyngeal 246 (1.6\%). For urogenital CT, $73.9 \%(n=16289)$ of the tests were performed in women, whereas these numbers were $42.4 \%(n=236)$ and $30.1 \%(n=74)$ for anorectal and oropharyngeal tests, respectively. 
The urogenital CT test positivity in men was $17.0 \%$ (251/1478) at the GP, $11.0 \%(235 / 2134)$ at the STI clinic, and 3.7\% (79/2128) in chlamydia screening. In women, these numbers were: GP 10.2\% (499/4871), STI clinic 13.4\% (341/2552), gynaecology 4.3\% (197/4567) and chlamydia screening $4.8 \%(206 / 4299)$.

\section{Contribution of different STI care providers}

Overall, GPs and chlamydia screening each accounted for $29 \%$ of all urogenital tests, whereas the STI clinic and gynaecologists each accounted for 21\% (figure 2A). The STI clinic and chlamydia screening tested the largest proportion of men (both 37\%), whereas GPs tested the largest proportion of women $(30 \%$, figure $2 \mathrm{~B})$.

Overall, 73.3\% (1326/1808) of the urogenital CT positives were observed in GP ( $n=750)$ and STI clinic ( $n=576$ ) patients. In men, GPs accounted for a small proportion of all tests, but these resulted in almost half of all positives.

\section{Evaluation of testing and test positivity between STI care providers}

After adjusting for age, test year and SES, chlamydia screening performed most tests, irrespective of sex (table 1). However, these tests yielded the lowest test positivity in men and women (the latter together with gynaecology, table 2). Although the STI clinic performed more tests in men than GPs, test positivity was lower in the former. In contrast, fewer tests were performed in women at the STI clinic as compared with the GP, but test positivity was higher in the former.

\section{Additional factors associated with testing and test positivity}

CT tests increased over time for both sexes at the STI clinic (table 1). More urogenital CT tests were performed in 22-29 than in 16-21 year-olds across all providers, irrespective of sex. GPs and gynaecologists performed more tests among individuals with lower SES.

In both sexes, GP test positivity decreased over time (table 2). In contrast, STI clinic test positivity increased in men. In women, younger age was associated with higher test positivity. Lower SES was associated with higher test positivity in women at the GP and gynaecologist. 
Figure 2: Contribution of the STI care providers in terms of number of urogenital Chlamydia trachomatis tests and positives, overall (A), by sex (B), by age (C) and by socio-economic status (D). CT screen, chlamydia screening programme; GP, general practitioner.

A.

Overall

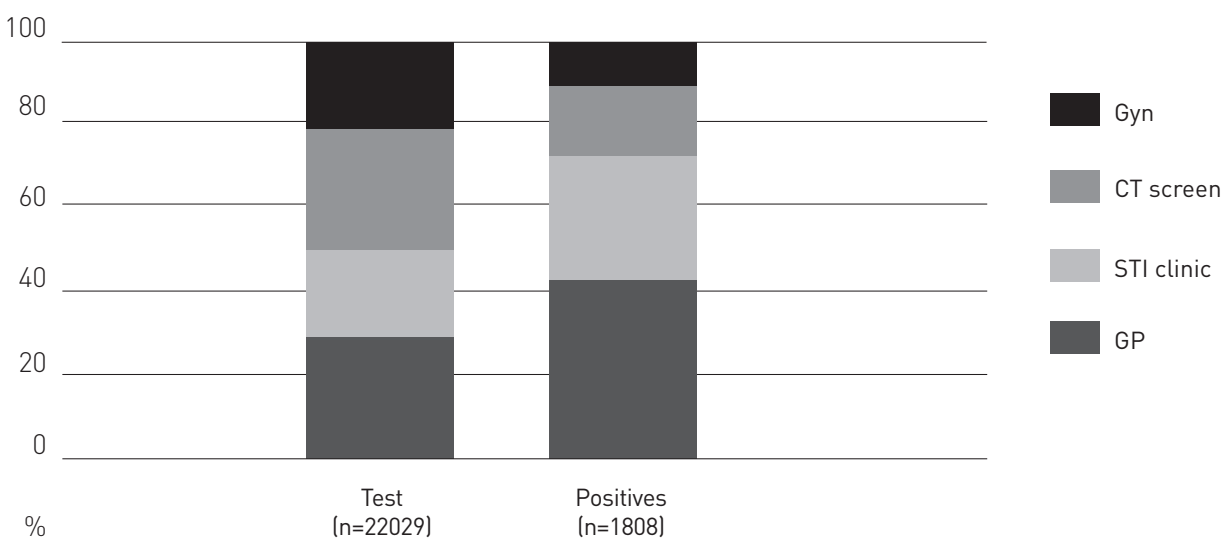

B.

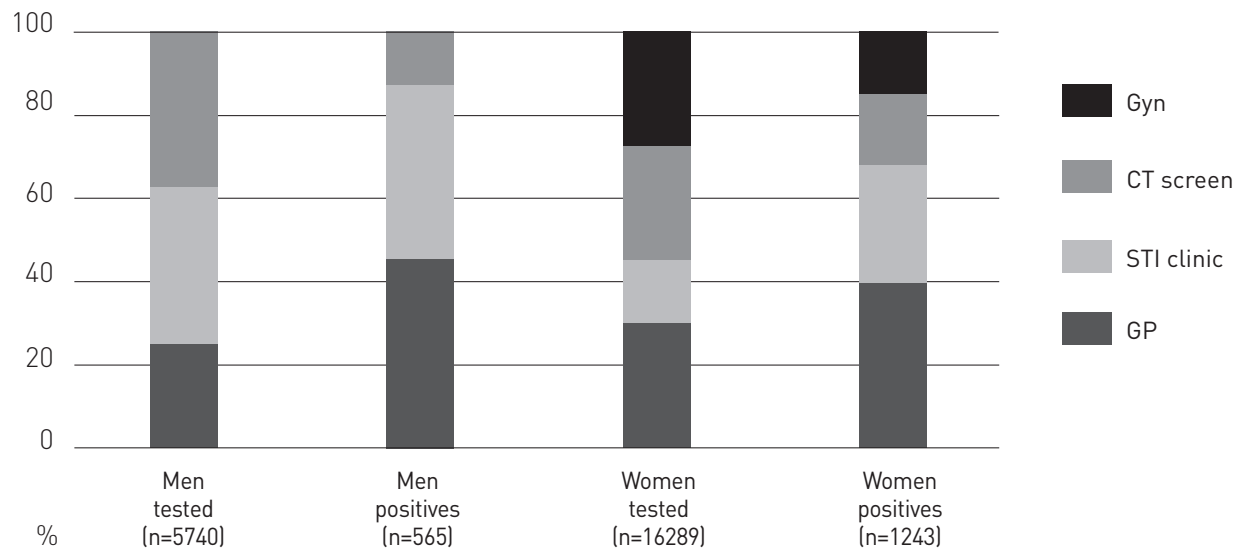


C.

By Age

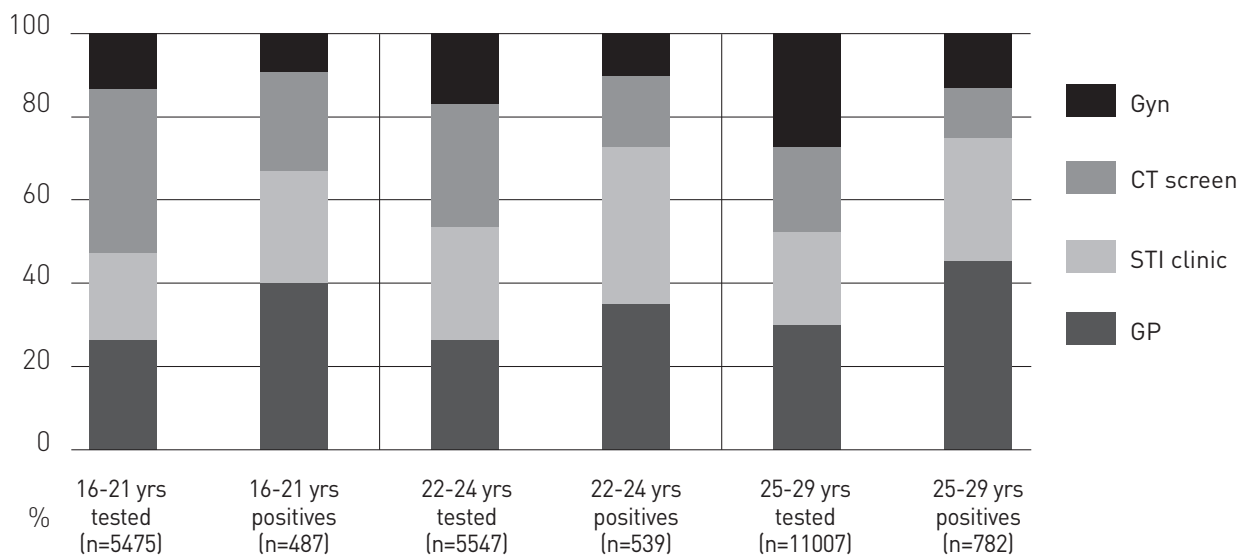

D.

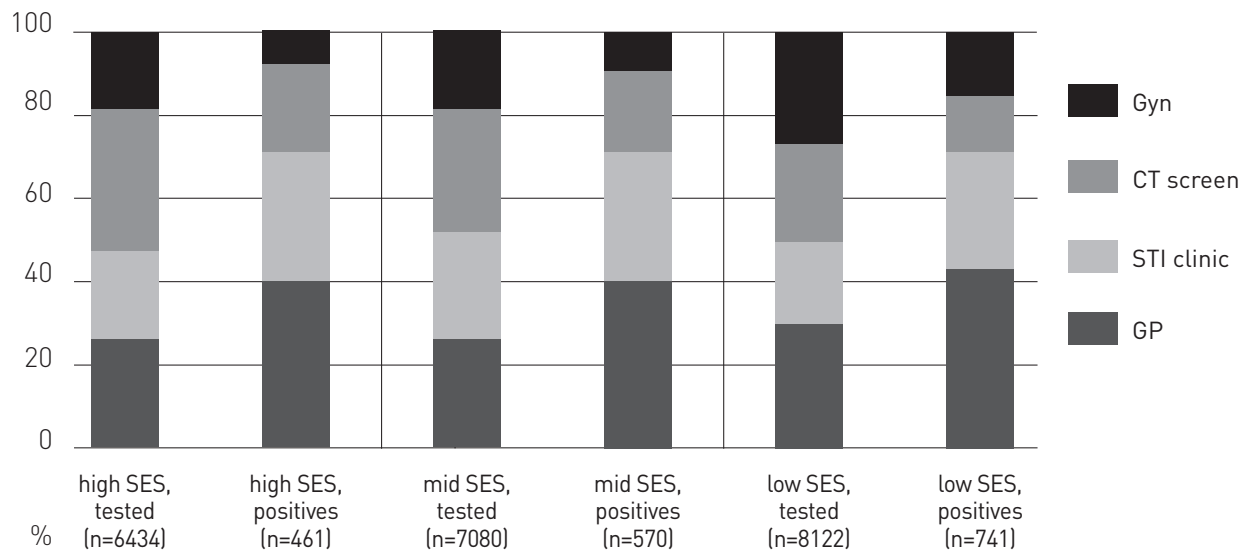




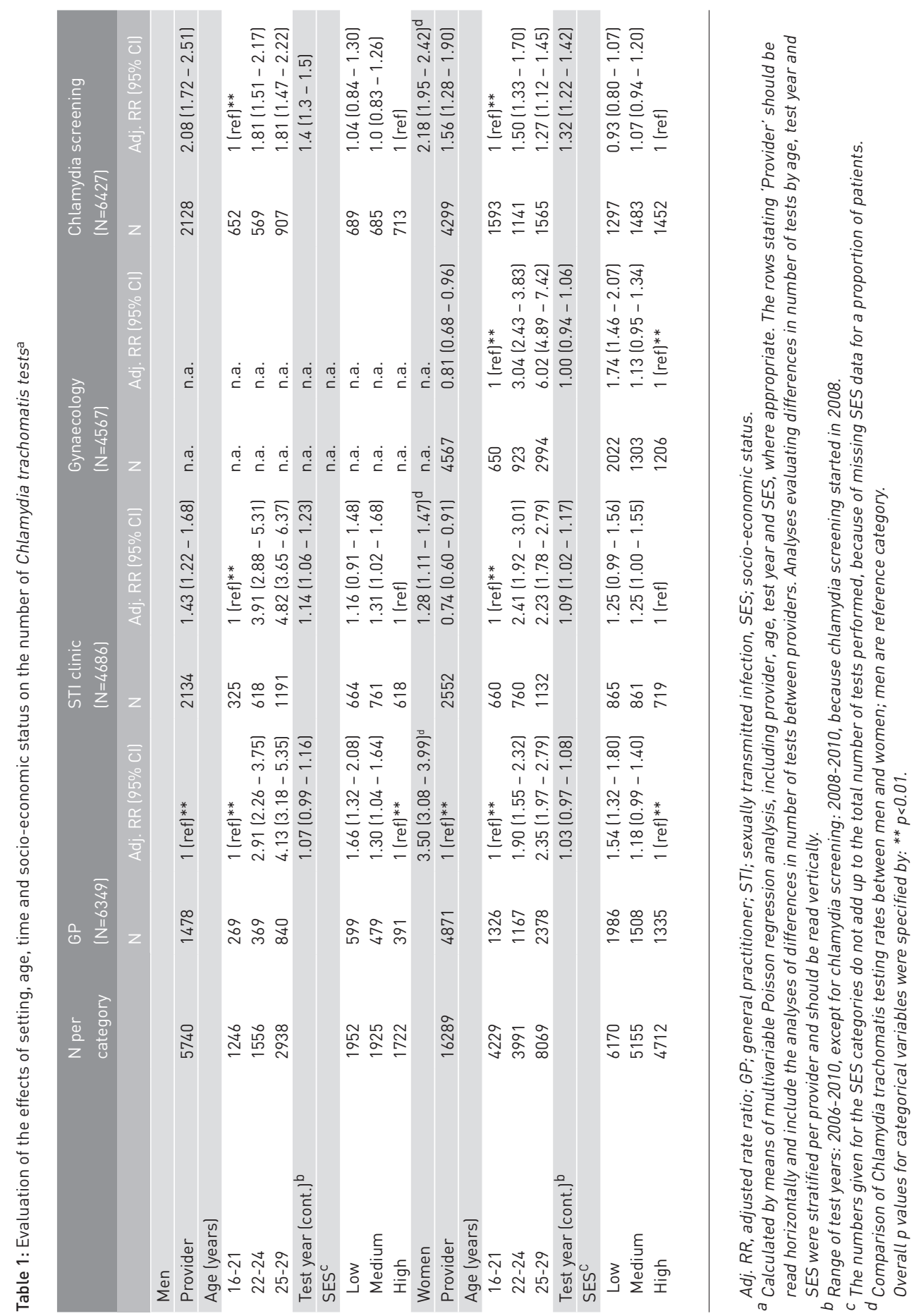




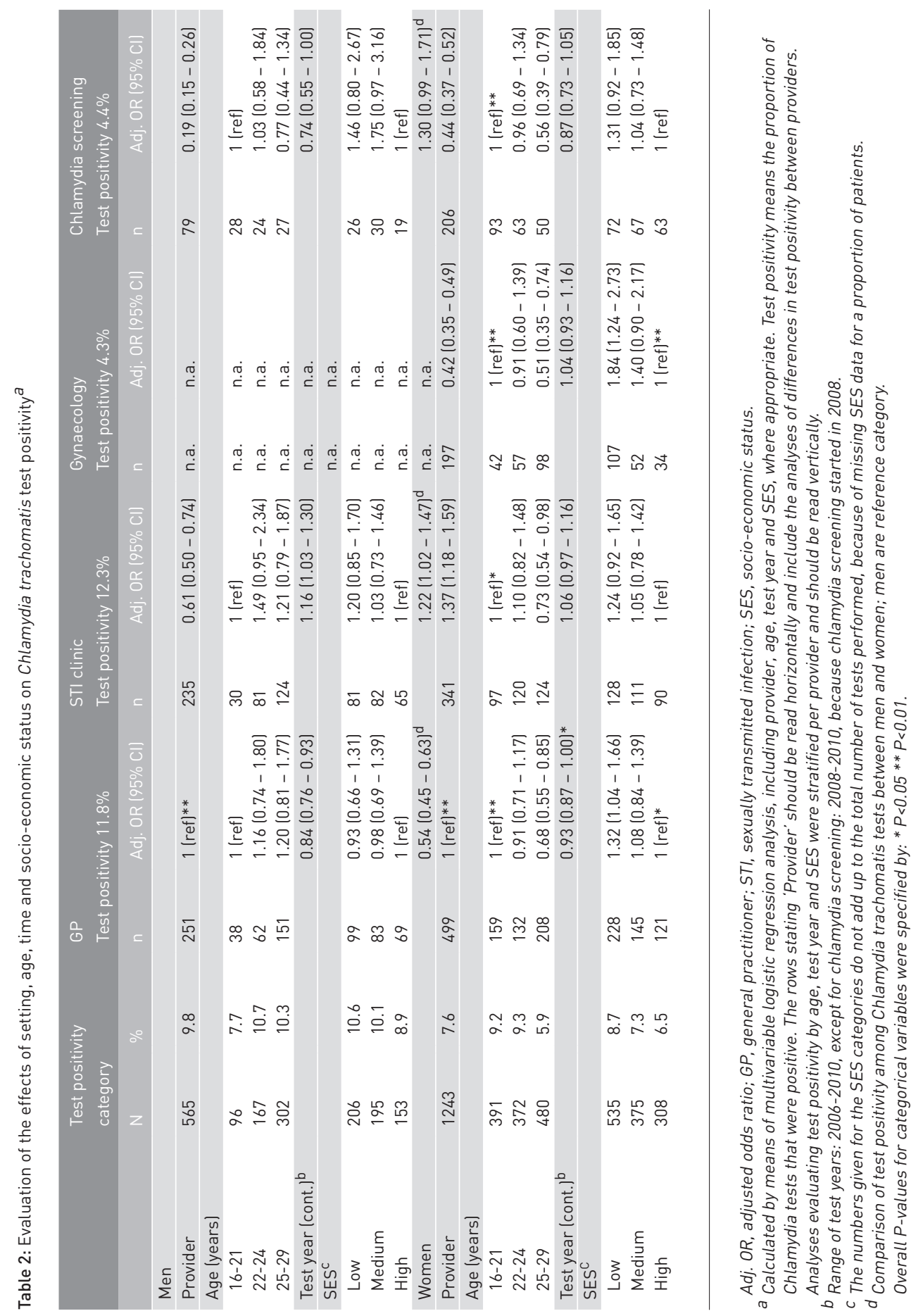




\section{Extra-genital CT test practice}

GPs performed no anorectal and three oropharyngeal tests (one in a man and two in women), and gynaecologists performed four anorectal and six oropharyngeal tests.

Within the STI clinic, $14.4 \%$ (789/5475) of all CT tests were extra-genital tests. Of these, $62.2 \%$ were performed in men. Most extra-genital tests were anorectal tests $165.2 \%$ in men and $77.9 \%$ in women).

In the STI clinic, anorectal CT test numbers increased over time for both men (OR: 1.45, 95\% $\mathrm{Cl} 1.31$ - 1.60) and women (OR: 1.34, 95\% Cl 1.20 - 1.50). This increase over time was more pronounced in men (OR: $1.29,95 \% \mathrm{Cl} 1.01$ - 1.65). Furthermore, more anorectal tests were performed among men or women aged 25-29 than among 16-year-old to 21-year-old men (OR: $2.78,95 \% \mathrm{Cl} 1.76-4.41$ ) or women (OR: 4.86, 95\% Cl $2.99-7.89$ ), respectively. More anorectal tests were performed among men with lower versus higher SES (OR: 1.55, 95\% Cl $1.04-2.32$ ). Of all extra-genital tests, $7.5 \%$ were positive, that is, 56 anorectal and 4 oropharyngeal. In $55.0 \%$ (33/60) of the extra-genital CT infections, no urogenital CT was diagnosed concurrently. None of the extra-genital CT tests performed outside the STI clinic were positive.

The STI clinic test positivity in men was: anorectal $10.0 \%(n=32)$ and oropharyngeal $2.3 \%(n=4)$. In women, these numbers were: anorectal $10.3 \%(n=24)$ and oropharyngeal $0 \%$.

For women, anorectal CT test positivity was lower in 25-29 than in 16-21 year-olds IOR: 0.23, 95\% $\mathrm{Cl} 0.07-0.76$ ). No associations were found between anorectal CT positivity and sex, test year or SES.

\section{Discussion}

We examined laboratory surveillance CT test practice data of 16-29 year-olds from regular STI care providers, that is, GPs, an STI clinic, gynaecologists, and chlamydia screening, covering one particular geographic region. GPs and the STI clinic together performed half of all urogenital CT tests, while accounting for $\sim 75 \%$ of CT positives. Gynaecologists performed over a quarter of all tests in women. Characteristics of tested populations were similar between STI care providers regarding age, but differed regarding sex and SES. Moreover, extra-genital CT testing was rarely performed by GPs and gynaecologists, although such testing revealed substantial positivity at the STI clinic in both sexes.

Here, we used a comprehensive dataset revealing CT test practice of both regular STI care providers and chlamydia screening, all serving residents of one geographic region. Moreover, the data included information about the anatomical testing site, enabling the evaluation of both urogenital and extra-genital CT test practice.

More detailed information regarding the characteristics (eg, sexual orientation) of the individuals 
tested was unknown, thus we could not compare CT test practice between, for example, heterosexual men and MSM. The proportion of MSM among all men tested in general practice is unknown, which could be evaluated in future studies.

Although the sampling methods between chlamydia screening and the STI clinic Imainly patient-collected), and GPs and gynaecologists (mainly clinician-collected) differed, this probably had negligible impact on test positivity, as both methods provide similar, valid results[21]. However, the sampling method, that is, patient-collected or clinician-collected, could have influenced the patients' threshold to seek testing and, thus, on test numbers between STI care providers[21].

High laboratory standards and the improbability that a patient is being tested at the same time at different laboratories, make double counting of data unlikely.

Finally, we could not correct for intra-class correlation to account for potential clustering within a specific STI care provider in our analyses, because the actual STI care provider who performed the test was unknown.

Our findings in men provide a contrast to a previous Dutch study, [7] who reported that GPs were responsible for $70 \%$ of CT diagnoses when evaluating GP and STI clinic CT test practice, whereas we observed a more equal distribution between these STI care providers. STI clinics target STI risk groups, such as individuals aged below 25 , and are therefore more relevant to our 16-yearold to 29-year-old population as compared to the previous study, where all age groups were included[11]. More importantly, GP data in this previous Dutch study were based on International Classification of Primary Care (ICPC-1) codes, with no specific CT codels), leading to less precise estimates than those made with our laboratory-confirmed data. Moreover, the present study demonstrates substantial differences regarding sex and that gynaecologists contribute significantly to CT test practice in women, alongside GPs and STI clinics.

GP and STI clinic urogenital CT test positivities were $>10 \%$ for both sexes, whereas test positivities of $<5 \%$ were found in both chlamydia screening and gynaecology. GPs and STI clinics test predominantly high-risk groups and patients with symptoms[22], whereas population-based screenings target a more general population, and gynaecologists mainly want to rule out CT in their clinical evaluations.

For GPs, test positivity decreased over time, while test numbers remained unchanged. A growing tendency for high-risk populations to visit an STI clinic rather than their GP could explain this trend, as reflected by an increase in test positivity in the former. However, we are only able to speculate about this, and future studies should explore this further and address the reason given for CT testing. 
The majority (70\%) of CT tests was performed in women, similar to the study by Dimech et al, who evaluated CT surveillance data from 15 Australian laboratories between 2008 and 2010[9]. A positive association between age and number of tests was shown across all STI care providers, irrespective of sex. In women, younger age was associated with higher test positivity. Similar associations were shown by Dimech et al. and other studies[23,24]. As argued in these papers, it appears difficult to reach at-risk women below 20 for testing; possible reasons are that they do not feel at risk, be unaware of the possible implications of CT on reproductive morbidity, are more likely to refuse testing or are less likely to consult their GP. This might explain why STI care providers test this high-risk group less than their somewhat older counterparts (20-29 years old) who have lower (yet still substantial) CT prevalence.

Although SES has been associated with CT test practice[10], we observed that SES was only associated with GP and gynaecologist CT test practice. The fact that only patients with a 'highrisk profile' are seen at the STI clinic and selected for chlamydia screening in Parkstad, could have weakened the association between SES and CT test practice.

Regarding extra-genital test practice, prevalence of anorectal CT was considerable $10 \%$ in both sexes attending the STI clinic), while oropharyngeal prevalence was much lower (0-3\%). Fifty-five per cent of extra-genital CT infections would have been missed if only urogenital testing had been performed. Moreover, a similar amount would be missed if extra-genital testing would be based on sexual history[13,15,16]. The fact that a substantial proportion of anorectal single infections are found in MSM supports the recommendation that MSM need to be tested at multiple anatomical locations, irrespective of sexual history[15]. With the debate surrounding the effectiveness of azithromycin for extra-genital CT is ongoing, such recommendation may also be applicable to women, yet more data on anorectal CT treatment effectiveness are needed.

Although extra-genital CT testing was predominantly performed at the STI clinic here, extragenital testing in this setting should nevertheless be further explored, as testing practices can differ considerably between STI clinics[14,25].

Extra-genital CT can be detected successfully using nucleic acid amplification tests (NAAT) on simple to obtain self-taken samples and both men and women are willing to test[21]. Strategies to increase the reach of these self-taken samples, and thereby lower barriers to accessing STI care for both patients and providers[26,27], include the use of internet-based programmes, e-health strategies and home-collection[28,29]. 


\section{Conclusions}

In conclusion, gynaecologists perform a substantial proportion of CT testing in women, although GPs and STI clinics are mainly responsible for CT diagnosis. For all STI care providers, age was associated with increased testing, but lower test positivity. Extra-genital CT testing in MSM and possibly high-risk women needs to be encouraged, especially among STI care providers outside the STI clinic, because almost no extra-genital tests were performed by GPs and gynaecologists in our sample.

A full picture of CT test practice in one particular region can highlight differences between STI care providers and the populations they test. Moreover, it may alert STI care providers which (sub) groups they need to test more often and give these providers knowledge on where to find certain expertise, such as on extra-genital testing. Ideally, it would enhance integrating the services between STI care providers in the region. Thereby, evaluations such as provided here can help optimise CT test practice and could improve the cooperation between STI care providers, which is already in place in several countries (for example, between GUM clinics and GPs in the UK) [30].

\section{Key messages}

- In Dutch 16-year-old to 29-year-old men and women, three-quarters of Chlamydia trachomatis infections are diagnosed by general practitioners (GPS) and sexually transmitted infection (STI) clinics.

- Gynaecologists order as many chlamydia tests as STI clinics do, yet the proportion of testpositives (in women) tested by the former is much lower.

- Characteristics of the tested populations differ between STI care providers regarding sex and socio-economic status, but are similar for age.

- Extra-genital chlamydia testing is rarely performed by GPs and gynaecologists.

\section{Acknowledgements}

The authors thank the staff of the STI Clinic South Limburg, the Department of Medical Microbiology, Atrium Medical Centre Parkstad and the Dutch Chlamydia Screening Implementation (CSI) project group, especially IVF van den Broek, EEHG Brouwers, JSA Fennema, RH Koekenbier, ELM Op de Coul, LL Pars and SM van Ravesteijn, for their valuable contribution to the CSI data collection. Moreover, we want to thank Jane Banfield for critically reviewing and editing the English language in this paper. 


\section{References}

1. http://www.ecdc.europa.eu/en/publications/ Publications/0906_GUI_Chlamydia_-Control in_Europe.pdf, date last accessed:

11 June 2015.

2. Price MJ, Ades AE, De Angelis D, et al. Risk of pelvic inflammatory disease following Chlamydia trachomatis infection: analysis of prospective studies with a multistate model. Am J Epidemiol 2013;178:484-92.

3. Low N, Cassell JA, Spencer B, et al. Chlamydia control activities in Europe: cross-sectional survey. Eur J Public Health 2012;22:556-61.

4. Santella AJ, Pollack A, Harrison C, et al. Management rates of sexually transmissible infections by Australian general practitioners, 2000-2012. Sex Health 2014;11:52-7.

5. van den Broek IV, van Bergen JEAM, Brouwers EE, et al. Effectiveness of yearly, register based screening for chlamydia in the Netherlands: controlled trial with randomised stepped wedge implementation. BMJ 2012;345:e4316.

6. Macintosh M, McKee M. The English National Chlamydia Screening Programme: where next? Public Health 2013;127:681-3.

7. van den Broek IV, Verheij RA, van Dijk CE, et al. Trends in sexually transmitted infections in the Netherlands, combining surveillance data from general practices and sexually transmitted infection centers. BMC Fam Pract 2010;11:39.

8. NCSP data, CTAD Data Tables 2013 http://www.chlamydiascreening.nhs.uk/ps/ resources/data-tables/CTAD\%20Data\%20 Tables\%202013\%20Annual\%20data\%20\%20
for\%20publication_FINAL130614.pdf, date last accessed: 11 June 2015.

9. Dimech W, Lim MS, Van Gemert $\mathrm{C}$ et al. Analysis of laboratory testing results collected in an enhanced chlamydia surveillance system in Australia, 2008-2010. BMC Infect Dis 2014;14:325.

10. Crichton J, Hickman M, Campbell R, et al. Prevalence of chlamydia in young adulthood and association with life course socioeconomic position: birth cohort study. PLoS One 2014; 9:e104943.

11. https://www.soaaids.nl/sites/default/files/ pictures/Multidisciplinaire\%20richtlijn\%20 SOA\%20voor\%20de\%202e\%20lijn\%202012$13 \% 20 \% 281 \% 29 . p d f$, date last accessed, 29 January 2015.

12. Javanbahkt $M$, Gorbach $P$, Stirland $A$, et al. Prevalence and correlates of rectal Chlamydia and gonorrhea among female clients at sexually transmitted disease clinics. Sex Transm Dis 2012;39:917-22.

13. van Liere GAFS, Hoebe CJPA, Wolffs PF, et al. High co-occurrence of anorectal chlamydia with urogenital chlamydia in women visiting an STI clinic revealed by routine universal testing in an observational study; a recommendation towards a better anorectal chlamydia control in women. BMC Infect Dis 2014;14:274.

14. Patton ME, Kidd S, Llata E, et al. Extragenital gonorrhea and chlamydia testing and infection among men who have sex with men--STD Surveillance Network, United States, 2010-2012. Clin Infect Dis 2014; 58:1564-70. 
15. van Liere GAFS, Hoebe CJPA, Dukers-Muijrers NHTM. Evaluation of the anatomical site distribution of chlamydia and gonorrhoea in men who have sex with men and in high-risk women by routine testing: cross-sectional study revealing missed opportunities for treatment strategies. Sex Transm Infect 2014;90:58-60.

16. Peters RP, Dubbink JH, van der Eem L, et al. Cross-sectional study of genital, rectal, and pharyngeal Chlamydia and gonorrhea in women in rural South Africa. Sex Transm Dis 2014;41:564-9.

17. Van Bergen JEAM, Fennema JS, van den Broek IV, et al. Rationale, design, and results of the first screening round of a comprehensive, register-based, Chlamydia screening implementation programme in the Netherlands. BMC Infect Dis 2010;10:293.

18. van den Broek IV, Brouwers EE, Götz HM, et al. Systematic selection of screening participants by risk score in a Chlamydia screening programme is feasible and effective. Sex Transm Infect 2012;88:205-211.

19. Op de Coul EL, Götz HM, van Bergen JEAM, et al. Who participates in the Dutch Chlamydia screening? A study on demographic and behavioral correlates of participation and positivity. Sex Transm Dis 2012;39:97-103.

20. van Liere GAFS, Dukers-Muijrers NHTM, van Bergen JEAM, et al. The added value of chlamydia screening between 2008-2010 in reaching young people in addition to chlamydia testing in regular care; an observational study. BMC Infect Dis 2014;14:612.

21. van der Helm JJ, Hoebe CJPA, van Rooijen
MS, et al. High performance and acceptability of self-collected rectal swabs for diagnosis of Chlamydia trachomatis and Neisseria gonorrhoeae in men who have sex with men and women. Sex Transm Dis 2009;36:493-7.

22. van Bergen JEAM, Kerssens JJ, Schellevis $F G$, et al. Sexually transmitted infection health-care seeking behaviour in the Netherlands: general practitioner attends to the majority of sexually transmitted infection consultations. Int J STD AIDS 2007;18:374-9.

23. Kufeji O, Slack R, Cassell JA, Pugh S, Hayward A. Who is being tested for genital chlamydia in primary care? Sex Transm Infect 2003;79:234-236.

24. Hughes G, Williams T, Simms I, et al. Use of a primary care database to determine trends in genital chlamydia testing, diagnostic episodes and management in UK general practice, 1990-2004. Sex Transm Infect 2007; 83:310-3.

25. Koedijk FD, van Bergen JEAM, DukersMuijrers NHTM, et al. The value of testing multiple anatomic sites for gonorrhoea and chlamydia in sexually transmitted infection centres in the Netherlands, 2006-2010. Int J STD AIDS 2012;23:626-31.

26. Cunningham SD, Kerrigan DL, Jennings JM, Ellen JM. Relationships between perceived STD-related stigma, STD-related shame and STD screening among a household sample of adolescents. Perspect Sex Reprod Health 2009;41:225-30.

27. Hocking JS, Parker RM, Pavlin N, Fairley CK, Gunn JM. What needs to change to increase chlamydia screening in general practice in 
Australia? The views of general practitioners.

BMC Public Health 2008;8:425.

28. Theunissen KA, Hoebe CJPA, Crutzen R, et al.

Using intervention mapping for the develop-

ment of a targeted secure web-based

outreach strategy named SafeFriend, for

Chlamydia trachomatis testing in young

people at risk. BMC Public Health

2013;13:996.

29. Fuller SS, Mercer CH, Copas AJ et al. The SPORTSMART study: a pilot randomised controlled trial of sexually transmitted infection screening interventions targeting men in football club settings. Sex Transm Infect 2015;91:106-10.

30. Kelly C, Johnston J, Carey F. Evaluation of a partnership between primary and secondary care providing an accessible Level 1 sexual health service in the community. Int J STD AIDS 2014;25:751-7. 



$$
\begin{gathered}
\text { Section } \\
\text { extra-genital } \\
\text { testing }
\end{gathered}
$$





\section{Chapter 4}

Standard symptom- and sexual history-based testing misses anorectal Chlamydia trachomatis and Neisseria gonorrhoeae infections in swingers and men who have sex with men

Geneviève AFS van Liere, Christian JPA Hoebe, Anne-Marie Niekamp,

Femke DH Koedijk, Nicole HTM Dukers-Muijrers

Sexually Transmitted Diseases 2013; 40(4):285-9 


\section{Abstract}

\section{Introduction}

Currently, individuals at risk for sexually transmitted diseases (STDs) are tested extragenitally only if indicated, most often when there is a history of self-reported symptoms or self-reported anal sex. The sensitivity of such selective symptom- and sexual history-based testing for detection of anorectal STD has not been determined.

\section{Methods}

All men having sex with men (MSM) and swingers (heterosexual couples who have sex with other heterosexual couples and their self-identified heterosexual sex partners) attending our STD clinic (consults: $\mathrm{n=1690)}$ ) from January 2010 until February 2011 were universally tested for urogenital, anorectal and oropharyngeal Chlamydia trachomatis (CT) and Neisseria gonorrhoeae (NG) infections (STD). We compared STD prevalence at anorectal site based on universal versus selective testing.

\section{Results}

Sensitivity of selective symptom- and sexual history-based testing for anorectal STD was $52 \%$ for homosexual MSM, 40\% for bisexual MSM, 43\% for bisexual male swingers, $40 \%$ for heterosexual male swingers and $47 \%$ for female swingers.

\section{Discussion}

Universal testing STD clinic clients who were MSM and swinger yielded more than half of all anorectal STD infections and is more sensitive for identifying anorectal STD infections compared with selective testing. Universal testing may be a more effective strategy for interrupting the ongoing transmission in high risk sexual networks. 


\section{Introduction}

Sexually transmitted diseases (STDs) at extra-genital sites are common. Studies found anorectal STD in up to $21 \%$ of men having sex with men (MSM) [1-8] and women [8-13]. Early detection and treatment are critical strategies in STD control to prevent medical complications and reduce transmission [7]. Therefore, availability of an appropriate diagnostic test is essential. The highly sensitive and specific nucleic acid amplification tests (NAATs) are superior to culture for extragenital Chlamydia trachomatis (CT) and Neisseria gonorrhoeae (NG) detection [14].

US Centers for Disease Control guidelines advocate annual testing for CT and NG in sexually active women and/or women under 26 years 15 but make no recommendations on anatomic site-specific testing in this group. For sexually active MSM, US Centers for Disease Control guidelines advocate anatomic site-specific testing for CT and NG based on sexual history, that is, urogenital testing after insertive anal intercourse, anorectal testing after receptive anal intercourse, and oropharyngeal testing after oral intercourse. WHO guidelines for MSM and transgender individuals advocate periodic testing for asymptomatic urogenital and anorectal CT and NG [15]. Dutch guidelines recommend anorectal testing in those with a history of anal sex and/or symptoms as well as oropharyngeal testing following self-reported oral sex [16]. However, heterosexual men and women were not often tested anorectally and/or oropharyngeally $[8,17]$. Prior clinical studies document high rates of missed extra-genital CT/NG by urogenital testing only in MSM $[1,3-8]$ and women $[8-10,12,13]$. However, these studies relied on selective testing based on history of anal sex and/or symptoms. Anorectal CT/NG infections are very likely to be asymptomatic [1] and history of sexual behavior seems to be an unreliable triaging tool for anatomic site-specific testing[2]. Reliability may be compromised by underreporting of specific sexual practices, or improper history taking. Two other studies universally screened MSM for oropharyngeal CT/NG [18,19], and 1 study universally screened HIV positives for anorectal and oropharyngeal CT/NG [20].

To date there have been no studies comparing symptom- and/or sexual history-based testing with universal testing at anorectal site in MSM and swingers. Swingers are heterosexual couples who have sex with other heterosexual couples and their self-identified heterosexual sex partners. Swingers differ from nonswinging heterosexual adults by their sexual network, characterized by concurrent sexual partners and high rates of unprotected sex. This makes swingers more prone to CT/NG [21]. With current testing practices, many anorectal CT/NG cases are likely to be missed, resulting in underestimated prevalence and ongoing transmission in high-risk populations. In this study, we determined the sensitivity of symptom- and sexual history-based testing, compared with universal testing at anorectal site in MSM and female/ male swingers. 


\section{Methods}

The South Limburg Public Health Service's STD unit provides more than 6000 consults annually, offering free examination and treatment at 3 regional outpatient STD clinics.

\section{Study population}

Between January 2010 and February 2011, MSM and male and female swingers were universally tested for CT and NG at 3 anatomic sites -urogenital, anorectal and oropharyngeal-yielding a total of 1690 consults for analysis. Individuals younger than 18 years were excluded from analysis on medical ethical grounds. Risk-group allocation was performed routinely in all attendees at each consult, based on self-reported sexual behaviour, in accordance with national testing guidelines. Men having sex with men were defined as men who had sex with 1 or more men in the past 6 months. An individual was registered as a swinger when he or she reported to be part of a male-female couple, who as a couple had sex with other male-female couples, or when he or she reported to be a swinger's heterosexual sexpartner.

Although swingers, by definition, have a heterosexual orientation, their sexual behavior may include same-sex activities. Swingers form their sexual networks especially at physical venues, like swingers clubs or erotic parties, and virtual venues, for example, specific dating websites.

\section{Variables}

Specimens tested were self-collected vaginal swabs, anorectal swabs, oropharyngeal swabs and urine; and in a minority of cases clinician-collected urethral and cervical swabs. Specimens were processed at 2 regional laboratories using 2 different nucleic acid amplification assays (strand displacement amplification [ Becton Dickinson ProbeTec ET system, Sparks MD] and polymerase chain reaction [PCR; Roche Cobas Implicor, San Fransisco, CA], respectively). The rationale for using NAAT assays for testing extra-genital specimens is that they have demonstrated higher sensitivity compared with culture [22]. Each of the 2laboratories performed approximately half of all tests included in our study. Laboratory selection was based on the geographical location of the STD clinic, and independent of risk group, anatomic site tested, or day and month of testing. All NG-positive samples were confirmed with an in-house PCR[23]. Serum was tested for Treponema pallidum hemagglutination (TPHA) and HIV (anti-HIV[1/2], Axsym; Abbott Laboratories, Chicago, IL). Reactive samples were confirmed using Western blot (HIVblot 2.2; Genelabs Diagnostics, Science Park, Singapore), according to the manufacturer's protocol. Besides testing, each consult included the taking of a standardized medical and sexual history by trained study nurses, including demographic data, self-reported symptoms and sexual behavior in the preceding 6 months. All data were registered in an electronic patient registry. 


\section{Variables}

Risk group category was hierarchically constructed based on self-reported sexual behavior that associated with sexual preference and sex of the sexpartner(s). Each participant/attendee was allocated to 1 of the after nonoverlapping groups: MSM who only had sex with men lhomosexual MSM), MSM who had sex with both men and women (bisexual MSM), male swingers who had sex with both men and women (bisexual male swingers), male swingers who only had sex with women (heterosexual male swingers) and female swingers. Rectal symptoms were defined as any of the following: rectal discharge, bleeding, pain, redness, burning sensation or itch. Anal sex included insertive anal sex, receptive anal sex or both. Age $(<32,32-45,>45$ years $)$ and number of sex partners in the past 6 months $(<3,3-8,>8)$ were categorized in 3 groups, both based on tertile distributions. HIV status was defined as (tested) positive or tested negative. Syphilis status was defined as positive or negative, based on presence or absence of TPHA.

\section{Statistical analysis}

Prevalences of CT, NG and CT and/or NG (CT/NG) were calculated by dividing the number of positive tests by the total number of tests multiplied by 100 . Prevalences of urogenital, anorectal and oropharyngeal CT/NG were stratified and compared between risk group category using $\mathrm{X} 2$ test. Prevalence of anorectal CT/NG was further stratified for self-reported anal sex (yes/no), rectal symptoms (yes/no) and the combination of self-reported anal sex and/or rectal symptoms (yes/no), using the X2 test. The proportion detected (sensitivity) by symptom- and sexual history-based (selective) testing was assessed by dividing the number of anorectal CT/NG found by symptom- and sexual history-based testing by the number of anorectal CT/NG found by universal testing and multiplied by 100 . The proportion CT/NG missed by symptom- and sexual history-based testing was calculated by dividing the number of anorectal CT/NG without anal sex or rectal symptoms by the total number of anorectal $\mathrm{CT} / \mathrm{NG}$ found by universal testing multiplied by 100 . A $p$ value $<0.05$ was considered as statistically significant. Analyses were performed using SPSS version 17.0.0 (IBM Inc. Somers, New York, USA).

\section{Results}

\section{Baseline characteristics}

We included 1690 STD consults by 1052 individuals; these included 482 homosexual MSM, 78 bisexual MSM, 79 bisexual male swingers, 165 heterosexual male swingers and 248 female swingers. The overall median age was 43 years (interquartile range [IQR] 32-49], MSM were somewhat younger compared to heterosexual male swingers and female swingers (35 years [IQR 24-47] vs 45 years [IQR 39-50]). Most had Dutch nationality (77.1\%), this varied between $68.0 \%-90.4 \%$. Rectal symptoms were reported in $1.0 \%$ to $5.9 \%$ consults; most consults were by homosexual MSM and female swingers. Anal sex was reported in $26.1 \%$ to $52.6 \%$ consults. Men having sex with men reported anal sex more often compared to swingers (table 1). 


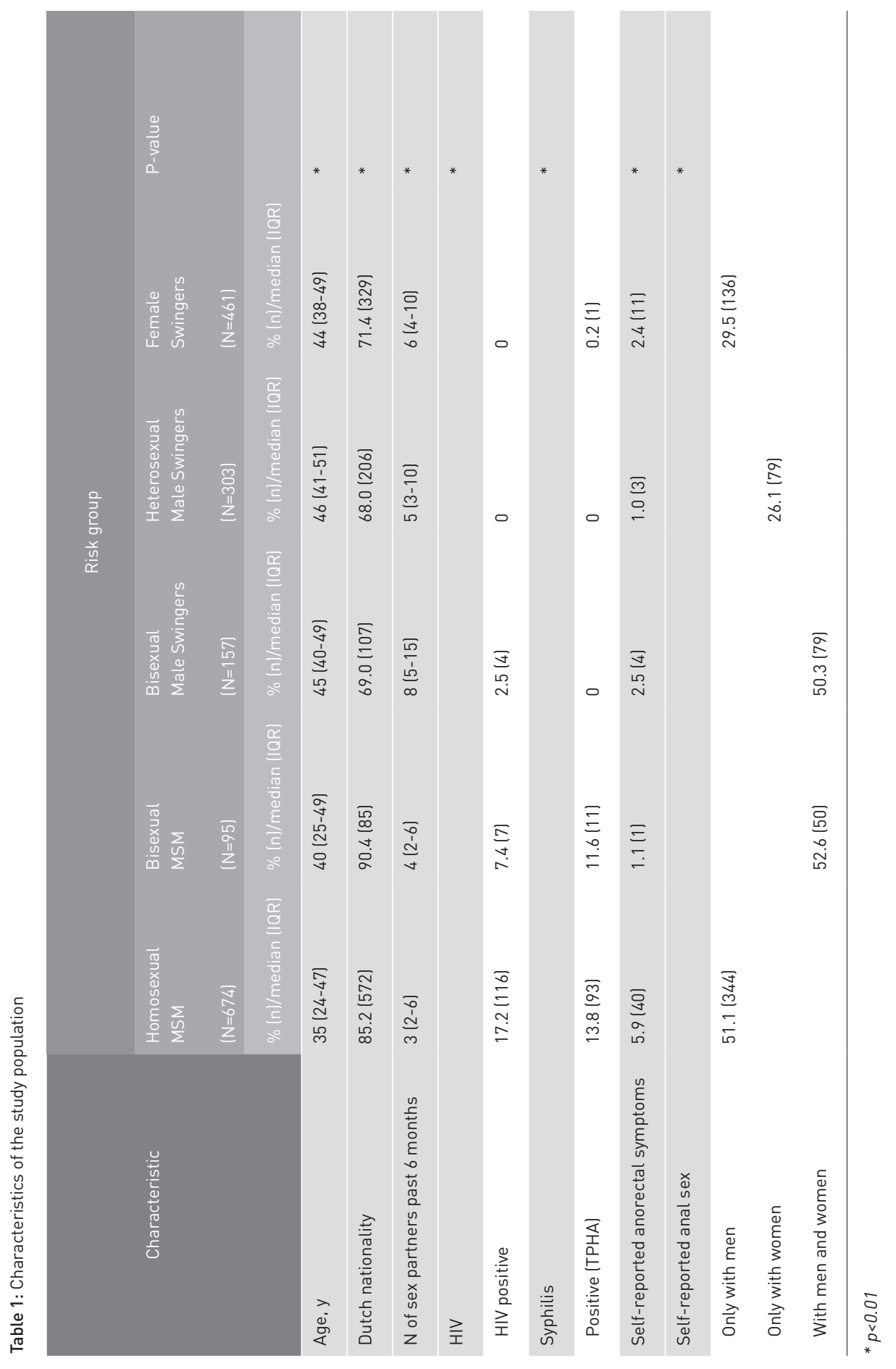




\section{Chlamydia and gonorrhoea prevalence and associated determinants after universal testing}

Prevalences of overall CT, NG and CT/NG varied by risk group category (table 2). Multiple-site infections (i.e. both anorectal and oropharyngeal) were scarcely observed (in 15 consultations). Prevalence of oropharyngeal CT/NG ranged by risk group category from $2.0 \%-5.1 \%$ ( $p=0.46$ ), prevalence of urogenital CT/NG ranged from 4.5\%-7.2\% ( $p=0.42)$ and prevalence of anorectal CT/ NG differed by risk group category (table 3). Prevalence of anorectal CT/NG was higher in consults with reported rectal symptoms (27.6\%) compared with consults without $(7.5 \%, \mathrm{p}<.001)$. Prevalence of anorectal CT/NG did not differ between consults with self-reported anal sex (9.6\%) and no self-reported anal sex $(7.3 \% \mathrm{p}=0.09)$. Prevalence also did not differ by presence or absence of the combination of anal sex and/or rectal symptoms (9.6\% vs.7.3\%, $p=0.09)$. In only 11 (0.7\%; confidence interval [Cl], 0.4\%-1.2\%) consults, a CT/NG coinfection was diagnosed.

Table 2: Prevalence of CT, NG and CT/NG at any site (urogenital, anorectal, oropharyngeal) by universal testing in 5 different risk group categories

\begin{tabular}{|c|c|c|c|c|c|c|}
\hline & $\begin{array}{l}\text { Homosexual } \\
\text { MSM }\end{array}$ & $\begin{array}{l}\text { Bisexual } \\
\text { MSM }\end{array}$ & $\begin{array}{l}\text { Bisexual Male } \\
\text { Swingers }\end{array}$ & $\begin{array}{l}\text { Heterosexual } \\
\text { Male Swingers }\end{array}$ & $\begin{array}{l}\text { Female } \\
\text { Swingers }\end{array}$ & P-value \\
\hline & $(\mathrm{N}=674)$ & $(N=95)$ & $(\mathrm{N}=157)$ & $(\mathrm{N}=303)$ & $(\mathrm{N}=461)$ & \\
\hline & $\%$ & $\%$ & $\%$ & $\%$ & $\%$ & \\
\hline CT & 10.5 & 10.5 & 7.0 & 5.3 & 8.5 & \\
\hline NG & 6.1 & 5.3 & 2.5 & 1.3 & 3.0 & * \\
\hline $\mathrm{CT} / \mathrm{NG}$ & 15.6 & 14.7 & 9.6 & 7.6 & 10.8 & $*$ \\
\hline
\end{tabular}

$* p<0.01$

\section{Anorectal chlamydia/gonorrhoea infections missed in standard symptom- and sexual history-based testing}

The overall proportion of anorectal CT/NG missed in symptom- and sexual history-based testing compared with non-selective, universal testing was 51.1\% (71/139, Cl 43-59\%) (table 3). Sensitivity of symptom- and sexual history-based testing for anorectal CT/NG differed across groups: $51.8 \%$ in homosexual MSM, $40.0 \%$ in bisexual MSM, $42.9 \%$ in bisexual male swingers, $40.0 \%$ in heterosexual male swingers and $47.1 \%$ in female swingers (table 3 ). Including self-reported rectal symptoms as an indication for anorectal testing yielded just 2 additional anorectal CT/NG diagnoses, somewhat lowering the proportion of missed anorectal CT/NG. The proportion of missed anorectal CT diagnoses was 55.0\% (33/60) in homosexual MSM, 42.9\% (3/7) in bisexual MSM, 50.0\% (3/6) in bisexual male swingers, $75.0 \%$ (3/4) in heterosexual male swingers and 


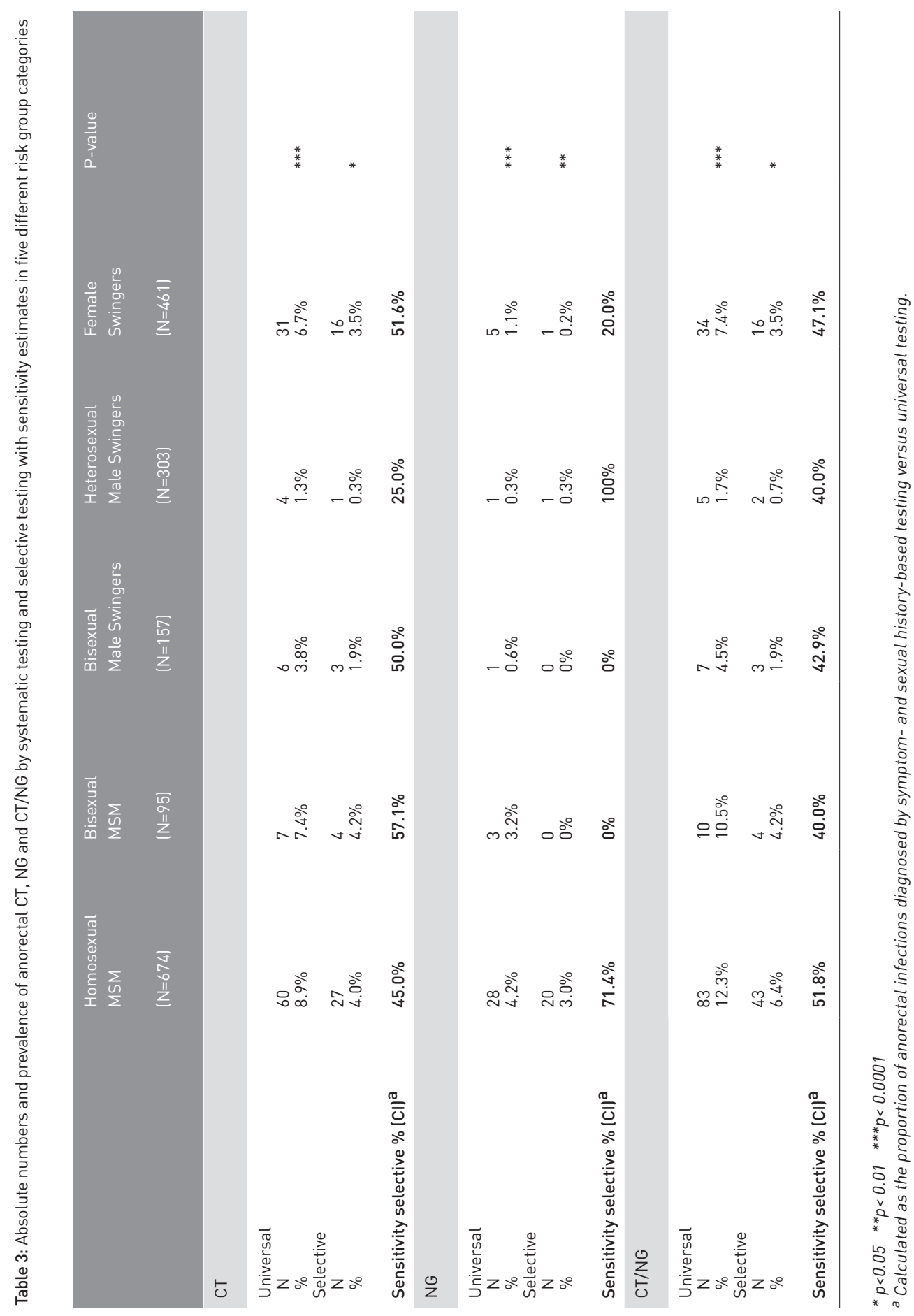


$48.4 \%(15 / 31)$ in female swingers $(p=0.84)$. For $N G$, this was $28.6 \%(8 / 28)$ in homosexual MSM, $100 \%(3 / 3)$ in bisexual MSM, $100 \%(1 / 1)$ in bisexual male swingers, $0 \%(0 / 1)$ in heterosexual male swingers and $80.0 \%(4 / 5)$ in female swingers ( $p=0.02$ ). For CT/NG this was $48.2 \%(40 / 83)$ in homosexual MSM, 60.0\% (6/10) in bisexual MSM, 57.1\% (4/7) in bisexual male swingers, $60.0 \%$ $(3 / 5)$ in heterosexual male swingers and $52.9 \%(18 / 34)$ in female swingers $(p=0.92)$.

\section{Discussion}

In MSM and female and male swingers sensitivity of symptom- and sexual history-based testing for detection of anorectal CT/NG was low. Prevalence of anorectal CT/NG was highest in MSM $(11 \%-12 \%)$ and female swingers $(7 \%)$ and the highest numbers of missed CT/NG were in these risk groups ( $49 \%$ - 60\% in MSM, 53\% in female swingers).

These findings add to the growing body of evidence supporting universal testing in MSM [7], and strongly suggest that universal testing may also be warranted for female swingers. Although literature on universal anorectal testing in women is scarce, our findings are supported by Barry et al, who found that $80 \%$ of the women who tested positive for anorectal CT did not report anal sex [9]. Presence of anorectal CT/NG in those men and women without a history of self-reported anal sex may be explained by underreporting, or alternative transmission routes not captured by routine history taking, for example anal-digital activity and use of sex toys. In women, autoinoculation by infected vaginal secretions [24] may also be a possibility, although scientific evidence for this is scarce.

Because using self-report of sexual behavior as indication for selective testing may be problematic, so is the use of self-reported symptoms. Several studies suggest that anorectal CT infections most often are asymptomatic [1,11], in our study, the prevalence of self-reported symptoms was very low $(5 \%)$.

Our study has several limitations. First, we had no sexual history data distinguishing between receptive and insertive anal sex, preventing us from performing analyses accounting for this difference. Limiting testing to those reporting receptive anal sex may yield a high prevalence of anorectal CT/NG in this selected group. However, as our study shows, this approach would likely be overselective, leading to even larger proportions of missed anorectal CT/NG.

Second, data on self-reported condom use were unreliable because of a high proportion of missing values in our records. Thus, we were unable to assess the association between condom use and anorectal CT/NG. A study by Tian et al. in the United States showed that condom use 
among heterosexuals practicing anal sex was low; $63 \%$ never used a condom during anal sex [25]. Data from a surveillance study in the Netherlands revealed that $69 \%$ of women did not use a condom during their last anal sexual contact with a casual partner, for men this was $45 \%$ [26].

Third, although our instructions on specimen collection were clear [22], we cannot entirely rule out the possibility of specimen contamination, for example via the urogenital-anorectal route. Fourth, the sensitivity of different NAAT in testing of oropharyngeal and anorectal samples is under discussion [22,27]. Sensitivity of strand displacement amplification and PCR was in some studies $[14,27]$ lower compared to other tests; this could have led to an underestimation of CT/ NG infections.

\section{Conclusions}

In conclusion, our data show low sensitivity of symptom- and sexual history-based testing for detection of anorectal CT/NG in high-risk groups. Therefore CT/NG control should include universal anorectal testing in MSM and female swingers for early detection and treatment of CT and NG in order to prevent medical complications and reduce further transmission.

\section{Acknowledgements}

The authors thank the staff of the Centre for Sexual Health South Limburg (STD clinic) for their valuable contribution to the data collection and MD V.H. Hackert, for editing assistance. 


\section{References}

1. Kent CK, Chaw JK, Wong W, al e. Prevalence of rectal, urethral, and pharyngeal chlamydia and gonorrhea detected in 2 clinical settings among men who have sex with men: San Francisco, California, 2003. Clin Infect Dis. 2005;41: 67-74.

2. Cachay ER, Sitapati A, Caperna J, Freeborn K, Lonergan JT, Jocson E, et al. Denial of risk behavior does not exclude asymptomatic anorectal sexually transmitted infection in HIV-infected men. PLoS One. 2009;4: e8504.

3. Ivens D, Macdonald K, Bansi L, al e.Screening for rectal chlamydia infection in a genitourinary medicine clinic. Int J STD AIDS. 2007;18: 404-406.

4. Annan NT, Sullivan AK, Nori A, al e. Rectal chlamydia--a reservoir of undiagnosed infection in men who have sex with men. Sex Transm Infect. 2009;85: 176-179.

5. Manavi K, McMillan A, Young $\mathrm{H}$. The prevalence of rectal chlamydial infection amongst men who have sex with men attending the genitourinary medicine clinic in Edinburgh. Int J STD AIDS. 2004;15: 162-164.

6. Geisler WM, Whittington WL, Suchland RJ, al e. Epidemiology of anorectal chlamydial and gonococcal infections among men having sex with men in Seattle: utilizing serovar and auxotype strain typing. Sex Transm Dis. 2002;29: 189-195.

7. Schachter J, Philip SS. Testing Men Who Have Sex With Men for Urethral Infection With Chlamydia trachomatis and Neisseria gonorrhoeae Is Only Half the Job, and We Need the Right Tools. Sex Transm Dis. 2011;38: 925-927.
8. Koedijk FD, van Bergen JE, Dukers-Muijrers $\mathrm{NH}$, van Leeuwen AP, Hoebe CJ, van der Sande MA. The value of testing multiple anatomic sites for gonorrhoea and chlamydia in sexually transmitted infection centres in the Netherlands, 2006-2010. Int J STD AIDS. 2012;23: 626-631.

9. Barry PM, Kent CK, Philip SS, al e. Results of a program to test women for rectal chlamydia and gonorrhea. Obstet Gynecol. 2010;115: 753-759.

10. Hunte T, Alcaide M, Castro J. Rectal infections with chlamydia and gonorrhoea in women attending a multiethnic sexually transmitted diseases urban clinic. Int J STD AIDS. 2010;21: 819-822.

11. Raychaudhuri M, Birley HD. Audit of routine rectal swabs for gonorrhoea culture in women. Int J STD AIDS. 2010;21: 143-144.

12. Sethupathi M, Blackwell A, Davies H. Rectal Chlamydia trachomatis infection in women. Is it overlooked? Int J STD AIDS. 2010;21: 93-95.

13. Peters RP, Nijsten N, Mutsaers J, Jansen CL, Morre SA, van Leeuwen AP. Screening of oropharynx and anorectum increases prevalence of Chlamydia trachomatis and Neisseria gonorrhoeae infection in female STD clinic visitors. Sex Transm Dis. 2011;38: 783-787.

14. Schachter J, Moncada J, Liska S, Shayevich C, Klausner JD. Nucleic acid amplification tests in the diagnosis of chlamydial and gonococcal infections of the oropharynx and rectum in men who have sex with men. Sex Transm Dis. 2008;35: 637-642.

15. Prevention and treatment of HIV and other 
sexually transmitted infections among men

who have sex with men and transgender people. Recommendations for a public health approach. WHO Guidelines for the management of sexually transmitted infections. 2011.

16. Venereologie NVvDe. Diagnostiek en Behandeling van Seksueel Overdraagbare Aandoeningen (SOA). 2011.

17. Vriend H, Koedijk F, Broek van den I, Veen van $M$, Coul Op de E, Sighem van A. Sexually transmitted infections, inlcuding HIV, in the Netherlands in 2010. Centre for Infectious Disease Control - National Institute for Public Health and the Environment (RIVM). 2011.

18. Morris SR, Klausner JD, Buchbinder SP, al e. Prevalence and incidence of pharyngeal gonorrhea in a longitudinal sample of men who have sex with men: the EXPLORE study. Clin Infect Dis. 2006;43: 1284-1289.

19. Templeton DJ, Jin F, Imrie J, al e. Prevalence, incidence and risk factors for pharyngeal chlamydia in the community based Health in Men (HIM) cohort of homosexual men in Sydney, Australia. Sex Transm Infect. 2008;84: 361-363.

20. Soni S, White JA. Self-Screening for Neisseria gonorrhoeae and Chlamydia trachomatis in the Human Immunode-ficiency Virus ClinicHigh Yields and High Acceptability. Sex Transm Dis. 2011;38: 1107-1109.

21. Dukers-Muijrers NH, Niekamp AM, Hoebe CJ, al e. Older and swinging; need to identify hidden and emerging risk groups at STI clinics. Sex Transm Infect. 2010;86: 315-317.

22. van der Helm JJ, Hoebe CJ, van Rooijen MS, Brouwers EE, Fennema HS, Thiesbrummel
HF, et al. High performance and acceptability of self-collected rectal swabs for diagnosis of Chlamydia trachomatis and Neisseria gonorrhoeae in men who have sex with men and women. Sex Transm Dis. 2009;36: 493-497.

23. Tabrizi SN, Unemo M, Limnios AE, Hogan TR, Hjelmevoll SO, Garland SM, et al. Evaluation of six commercial nucleic acid amplification tests for detection of Neisseria gonorrhoeae and other Neisseria species. J Clin Microbiol. 2011;49: 3610-3615.

24. Kinghorn GR, Rashid S. Prevalence of rectal and pharyngeal infection in women with gonorrhoea in Sheffield. Br J Vener Dis. 1979;55: 408-410.

25. Tian LH, Peterman TA, Tao G, al e. Heterosexual anal sex activity in the year after an STD clinic visit. Sex Transm Dis. 2008;35: 905-909.

26. Bakker F, Graaf de H, Haas de S, Kedde H, Kruijer H, Wijsen C. Seksuele Gezondheid in Nederland 2009. Rutgers Nisso Groep. 2009.

27. Bachmann LH, Johnson RE, Cheng $H$, Markowitz LE, Papp JR, Hook EW, 3rd. Nucleic acid amplification tests for diagnosis of Neisseria gonorrhoeae oropharyngeal infections. J Clin Microbiol. 2009;47: 902-907. 



\title{
Chapter 5
}

\begin{abstract}
Evaluation of the anatomical site distribution of chlamydia and gonorrhoea in men who have sex with men and in high-risk women by routine testing: cross-sectional study revealing missed opportunities for treatment strategies
\end{abstract}

Geneviève AFS van Liere, Christian JPA Hoebe, Nicole HTM Dukers-Muijrers

Sexually Transmitted Infections 2014 ;90(1):58-60 


\begin{abstract}

\section{Introduction}

Current strategies for controlling non-urogenital chlamydia and gonorrhoea are not uniform. It is assumed that present anorectal/oropharyngeal infections are coincidentally treated with urogenital infections. However, it is not clear whether this control strategy is effective. To inform current debate, we evaluated the anatomical site distribution of chlamydia and gonorrhoea by routine testing in men who have sex with men (hereafter men) and in highrisk women (prostitutes and swingers, hereafter women).
\end{abstract}

\title{
Methods
}

Between January 2010 and November 2012, all men (n=2436) and women ( $n=1321$ ) attending our sexually transmitted infection clinic were routinely tested for anorectal, oropharyngeal and urogenital Chlamydia trachomatis and Neisseria gonorrhoeae. Data were collected on demographics and sexual behavior.

\section{Results}

Overall chlamydia positivity was $10.4 \%(254 / 2436)$ in men and $7.0 \%(92 / 1321)$ in women, for gonorrhoea this was $6.3 \%(154 / 2436)$ and $3.1 \%(41 / 1321)$ respectively. Isolated nonurogenital infections accounted to $76 \%$ of all infections in men and for up to $59 \%$ of all infections in women. For combined urogenital and anorectal infections, this amounted to $14 \%$ for men and up to $54 \%$ for women.

\section{Discussion}

Testing only for non-urogenital infections is insufficient, as it overlooks many infections. The use of coincidental treatment is therefore a suboptimal control strategy in high-risk groups for halting complications and transmission. There is an urgent need to optimise the testing guidelines for chlamydia and gonorrhoea at different anatomical sites. 


\section{Introduction}

Anorectal and oropharyngeal chlamydia and gonorrhoea are common in men who have sex with men (1-24\%)[1-4] and in high-risk women (1-15\%)[2,3,5,6]. Insight into the anatomical site distribution of sexually transmitted infections (STI) is important to understanding the appropriateness of current strategies for diagnosing and treating anorectal and oropharyngeal chlamydia and gonorrhoea, in order to halt complications and transmission. One common strategy includes coincidental concurrent treatment based solely on the identification of urogenital chlamydia and gonorrhoea, given that few STI clinics[2] and even fewer GPs (0.1\%)[7] routinely test for nonurogenital chlamydia and gonorrhoea. This strategy can be effective only if (1) non-urogenital infections occur mainly in the presence of urogenital infections and (2) the same treatment regimen is appropriate for the different anatomical sites. Previous studies have demonstrated that symptom-based testing is not an effective strategy, but history based testing may be and these studies seem to have informed much of current practice[1,2,4-6]. Studies based on symptom- and sexual history-based testing (selective testing) have revealed high shares of combined urogenital and anorectal infections (chlamydia 63-90\%, gonorrhoea 56-73\%) in highrisk women attending STI clinics[2,3,5,6] and high shares of isolated anorectal chlamydia (54-91\%) and gonorrhoea $(21 \%)[1,2,4]$ for all diagnosed infections in men who have sex with men. Up till now, only three studies have applied routine universal testing i.e. not based on symptoms or sexual history. One study examined the sensitivity of symptom- and sexual historybased testing for anorectal STI: sensitivity was low (40-52\%) and this procedure is likely to miss a substantial number of anorectal infections[3]. Two studies examined oropharyngeal STI in men who have sex with men and reported substantial shares of isolated oropharyngeal chlamydia $(68 \%)$ and gonorrhoea $(98 \%)[8,9]$. Moreover, the appropriate treatment for anorectal chlamydia is the subject of ongoing debate. Several studies have reported substantial microbial failure rates of up to $21 \%$ for single-dose azithromycin $(1.0 \mathrm{~g})$ in anorectal chlamydia[10]. Guidelines from the Netherlands and the United Kingdom currently advocate seven days of doxycycline 100 $\mathrm{mg}$ twice daily as a first-line treatment, and azithromycin is advocated as equal treatment by the US Centers for Disease Control.

To inform the current debate on appropriate strategies for controlling anorectal and oropharyngeal infections, we evaluated the anatomical site distribution of chlamydia and gonorrhoea by performing routine universal testing at urogenital, anorectal and oropharyngeal site in men who have sex with men and in high-risk women. 


\section{Methods}

From January 2010 to November 2012, all men who have sex with men and all high-risk women aged 18 years or older who attended the South Limburg Public Health Service's STI clinic were routinely tested for urogenital, anorectal and oropharyngeal Chlamydia trachomatis and Neisseria gonorrhoeae. In total, $17 \%(n=776)$ of patient population were not tested at all anatomical sites. Coverage varied by risk group and time; $88-95 \%$ in men who have sex with men, $91-95 \%$ in swingers and $25-46 \%$ in prostitutes. A total of 2436 consultations by 1218 men and 1321 consultations by 516 women were used for analyses. Men who have sex with men (hereafter men) were defined as men who had engaged in sex with one or more men in the past six months. High-risk women (hereafter women) included prostitutes and swingers[3]. Swingers were defined as women who were part of a male-female couple who as a couple had sex with other male-female couples and their self-identified heterosexual sex partners. Prostitutes were defined as women who reported to have had sex for money in the past 6 months. Of the 1321 women, $18.7 \%$ were prostitute $(n=247)$ and $81.3 \%$ were swinger $(n=1074)$. At our STI clinic there is no current test of cure practice and individuals are not retested within 3 months. The median number of days between repeat tests was 171 (IQR 105-228). The specimens tested consisted of self-collected vaginal swabs or urine, anorectal swabs and clinician-collected oropharyngeal swabs[3]. Specimens were processed at two regional laboratories using three different nucleic acid amplification assays (NAATs) (SDA, Becton Dickinson ProbeTec ET system, Maryland, USA and PCR, Cobas Amplicor, Roche, California, USA and PCR, Cobas 4800, Roche, California, USA). Serum was tested for Treponema pallidum hemagglutination (TPHA) and HIV.

\section{Statistical analysis}

Variables were constructed according to the following mutually exclusive categories: isolated anorectal (including combined anorectal, oropharyngeal infection), combined urogenital and anorectal (including combined urogenital, anorectal and oropharyngeal infection), isolated oropharyngeal and isolated urogenital infection (including combined urogenital, oropharyngeal infection). Chi square tests were used to compare the distribution of infections across the various anatomical sites, as well as across demographic and behavioural factors for men and women. Analyses were performed using SPSS V.17.0.0 (IBM, Somers, New York, USA). The Medical Ethics Committee of Maastricht University (Identification number 11-4-108) approved the study.

\section{Results}

Of the 3757 men and women, the median age was 37 years (IQR: 25-47 years) for men and 43 years (IQR: $36-49$ years) for women. The majority (>95\%) were Caucasian. Men reported having 
had a median of four sex partners in the past six months (IQR: 2-10), while women reported seven (IQR: 4-13). The prevalence of HIV and syphilis was $12.5 \%$ and $15.3 \%$, respectively, for men, and $0 \%$ and $0.5 \%$, respectively, for women. The prevalence of chlamydia (ie, a positive result in at least one of three tested anatomical sites) was $10.4 \%$ (254/2436) for men and 7.0\% (92/1321) for women. For gonorrhoea, the prevalence rates were 6.3\% (154/2436) and 3.1\% (41/1321), respectively. Chlamydia and gonorrhoea co-infection was present in $1.2 \%(n=30)$ of men and $0.2 \%$ of women ( $n=3$ ). Overall, $23-76 \%$ of all infections were isolated non-urogenital (table 1).

Table 1: Prevalence of urogenital, anorectal and oropharyngeal chlamydia and gonorrhoea and anatomical site distribution of chlamydia and gonorrhoea in men who have sex with men and in high-risk women by routine universal anorectal, urogenital and oropharyngeal testing.

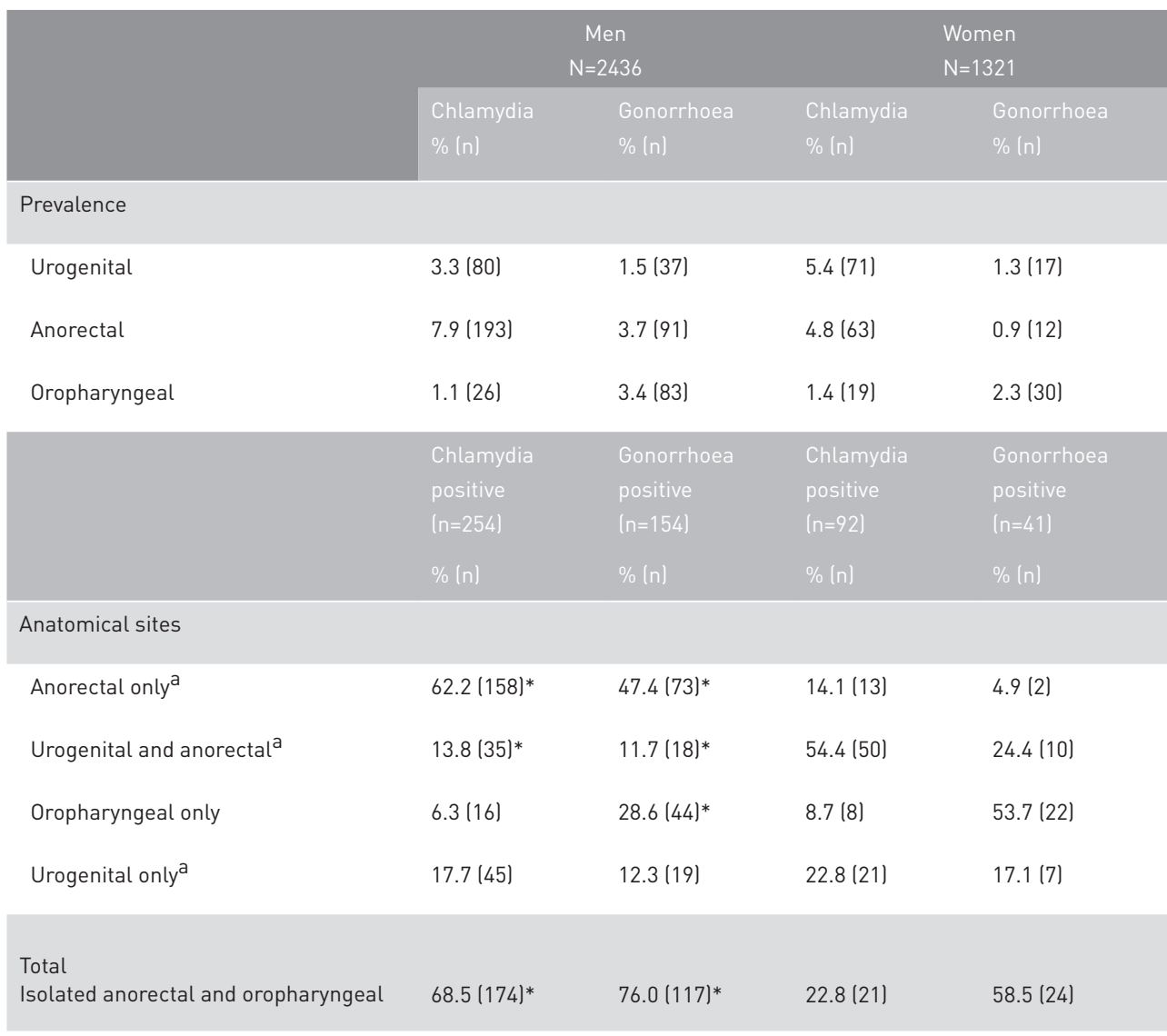

* $p<0.05$ Compared with women.

a Including oropharyngeal infection when present. 


\section{Discussion}

This is the first study to provide a systematic assessment of the anatomical site distribution of chlamydia and gonorrhoea with routine universal urogenital, anorectal and oropharyngeal testing in men who have sex with men and in high-risk women. The proportion of isolated nonurogenital infections amongst all chlamydia and gonorrhoea infections was substantial for men (up to $76 \%$ ) and women (up to $59 \%$ ). Urogenital testing alone is thus not an appropriate diagnostic strategy for detecting anorectal and oropharyngeal chlamydia and gonorrhoea in these high-risk groups. Treatment of urogenital infections alone overlooks many infections in high-risk groups such as studied here, and it therefore excludes many patients from proper treatment. The high proportion of isolated oropharyngeal gonorrhoea in women is notable. The relative importance of these oropharyngeal infections in the burden of disease (whether clinical or otherwise) is unclear, as is their share in transmission and the speed with which they can be cleared. In a previous study, we showed that the sensitivity of anorectal testing based on behavioural indications or symptoms is low[3]. Combined urogenital and anorectal chlamydia occurred in $14 \%$ of men and in $54 \%$ of women. Our results are consistent with previous reports that demonstrate high shares of isolated infections in men and high shares of combined infections in women[1,2,4-6]. It is not known whether concurrent treatment with azithromycin would constitute sufficient treatment for such combined infections. Some evidence suggests that azithromycin is suboptimal in treating anorectal chlamydia[10]. Our study sample did not include unique individuals only, which may be a limitation. However, restricting to first consultations in analysis gave similar results, therefore we expect bias to be minimal. Sensitivity and specificity of NAATs are high, although not $100 \%$. False positives may occur in lower-prevalence populations for example in the case of low prevalence of oropharyngeal STI. Furthermore, we could not completely rule out the possibility of autoinoculation by infected vaginal secretions in women. However, regardless of transmission route, anorectal infections can cause further STI transmission. Although our instructions on specimen collection were clear, we cannot entirely rule out the possibility of specimen contamination, for example, via the urogenital-anorectal route. Prostitutes had relatively low coverage. However, this group comprised a small part (19\%) of women and the anatomical site distributions of prostitutes and swingers were similar (data not shown). This may indicate that possible bias due to incomplete testing in prostitutes is minimal. However, it also warrants more research in prostitutes. The results of this study suggest routine universal testing could be preferable for high-risk clients of STI clinics. Although we can not extrapolate these results to other populations and care settings, our study demonstrates the need for further research in diverse settings to determine the need for multiple anatomical site testing.

\section{Acknowledgements}

We acknowledge the contributions of the staff of the STI clinic South Limburg in collecting the data. 


\section{References}

1. Peters RP, Verweij SP, Nijsten N, Ouburg S, Mutsaers J, Jansen CL, et al. Evaluation of sexual history-based screening of anatomic sites for chlamydia trachomatis and neisseria gonorrhoeae infection in men having sex with men in routine practice. BMC Infect Dis.11: 203.

2. Koedijk FD, van Bergen JE, Dukers-Muijrers $\mathrm{NH}$, van Leeuwen AP, Hoebe CJ, van der Sande MA. The value of testing multiple anatomic sites for gonorrhoea and chlamydia in sexually transmitted infection centres in the Netherlands, 2006-2010. Int J STD AIDS.23: 626-631.

3. van Liere GA, Hoebe CJ, Niekamp AM, Koedijk FD, Dukers-Muijrers NH. Standard Symptomand Sexual History-Based Testing Misses Anorectal Chlamydia trachomatis and Neisseria gonorrhoeae Infections in Swingers and Men Who Have Sex With Men. Sex Transm Dis.40: 285-289.

4. Kent CK, Chaw JK, Wong W, Liska S, Gibson S, Hubbard G, et al. Prevalence of rectal, urethral, and pharyngeal chlamydia and gonorrhea detected in 2 clinical settings among men who have sex with men: San Francisco, California, 2003. Clin Infect Dis. 2005;41: 67-74.

5. Peters RP, Nijsten N, Mutsaers J, Jansen CL, Morre SA, van Leeuwen AP. Screening of oropharynx and anorectum increases prevalence of Chlamydia trachomatis and Neisseria gonorrhoeae infection in female STD clinic visitors. Sex Transm Dis.38:783-787.

6. Barry PM, Kent CK, Philip SS, Klausner JD. Results of a program to test women for rectal chlamydia and gonorrhea. Obstet Gynecol. 115: 753-759.
7. Dukers-Muijrers NH, van Liere GA, Hoebe CJ. Re-screening Chlamydia trachomatis positive subjects: a comparison of practices between an STI clinic, general practitioners and gynaecologists. Sex Transm Infect.89: 25-27.

8. Morris SR, Klausner JD, Buchbinder SP, Wheeler SL, Koblin B, Coates T, et al. Prevalence and incidence of pharyngeal gonorrhea in a longitudinal sample of men who have sex with men: the EXPLORE study. Clin Infect Dis. 2006;43: 1284-1289.

9. Templeton DJ, Jin F, Imrie J, Prestage GP, Donovan B, Cunningham PH, et al. Prevalence, incidence and risk factors for pharyngeal chlamydia in the community based Health in Men (HIM) cohort of homosexual men in Sydney, Australia. Sex Transm Infect. 2008;84: 361-363.

10. Hathorn E, Opie C, Goold P. What is the appropriate treatment for the management of rectal Chlamydia trachomatis in men and women? Sex Transm Infect.88: 352-354. 



\section{Chapter 6}

High co-occurrence of anorectal chlamydia with urogenital chlamydia in women visiting an STI clinic revealed by routine universal testing in an observational study; a recommendation towards a better anorectal chlamydia control in women

Geneviève AFS van Liere, Christian JPA Hoebe, Petra FG Wolffs, Nicole HTM Dukers-Muijrers 


\section{Abstract}

\section{Introduction}

Symptom- and sexual history-based testing i.e., testing on indication, for anorectal sexually transmitted infections (STIs) in women is common. Yet, it is unknown whether this strategy is effective. Moreover, little is known about alternative transmission routes i.e. by fingers/ toys. This study assesses anorectal STI prevalence and infections missed by current testing practice, thereby informing the optimal control strategy for anorectal STIs in women.

\section{Methods}

Women ( $\mathrm{n}=663$ ) attending our STI-clinic between May 2012-July 2013 were offered routine testing for anorectal and urogenital Chlamydia trachomatis and Neisseria gonorrhoeae. Data were collected on demographics, sexual behaviour and symptoms. Women were assigned to one of the categories: indication (reported anal sex/symptoms), fingers/toys lonly reported use of fingers/toys), or without indication.

\section{Results}

Of women, $92 \%$ ( $n=654)$ participated. There were 203 reports $(31.0 \%)$ of anal sex and/or symptoms (indication), 48 reports (7.3\%) of only using fingers/toys (fingers/toys), and 403 reports (61.6\%) of no anal symptoms, no anal sex and no anal use of fingers/toys (without indication). The overall prevalence was $11.2 \%$ (73/654) for urogenital chlamydia and $8.4 \%$ (55/654) for anorectal chlamydia. Gonorrhoea infections were not observed. Prevalence of anorectal chlamydia was $7.9 \%(16 / 203)$ for women with indication and $8.6 \%$ (39/451) for all other women $(P=0.74)$. Two-thirds (39/55) of anorectal infections were diagnosed in women without indication. Isolated anorectal chlamydia was rare $(n=3)$ : of all women with an anorectal infection, $94.5 \%$ (52/55) also had co-occurrence of urogenital chlamydia. Of all women with urogenital chlamydia, $71.2 \%(52 / 73)$ also had anorectal chlamydia.

\section{Discussion}

Current selective testing on indication of symptoms and sexual history is not an appropriate control strategy for anorectal chlamydia in women visiting an STI clinic. Routine universal anorectal testing is feasible and may be a possible control strategy in women. Yet costs may be a problem. When more restricted control measures are preferred, possible alternatives include (1) anorectal testing only in women with urogenital chlamydia (problem: treatment delay or loss to follow up), and (2) direct treatment for urogenital chlamydia that is effective for anorectal chlamydia as well. 


\section{Introduction}

Chlamydia trachomatis $(\mathrm{Ct})$ and Neisseria gonorrhoeae $(\mathrm{Ng})$ are the most prevalent bacterial sexually transmitted infections (STIS) in women in high income countries and have major public health consequences [1-3]. In addition to infection of the urogenital tract, chlamydia and gonorrhoea can also cause anorectal infections in women. Previous studies of women who visited an STI clinic or a department of genitourinary medicine found anorectal chlamydia in up to $18 \%$ [2,4-11] of them and gonorrhoea in up to $13 \%$ of them [4-10]. However, guidelines in UK, US and the Netherlands do not recommend routine anorectal testing, but restricted testing in people who are in high-risk groups, report anal sexual behaviour, or have anal symptoms [12], i.e., selective testing on indication [12,13]. This is in contrast to urogenital testing, which is a routine procedure in STI care services in these countries. Nucleic acid amplification tests (NAATs) are the most sensitive tests for the screening and diagnosis of genital chlamydial infections to date, and their use is accepted and recommended for anorectal infections as well [14]. The impact of anorectal infections in women on population (public health) and individual (clinical) level are yet unknown. However, it is suggested that treatment of anorectal infections in women can help limit the spread of STI in the population [4-6] and can reduce complications in infected individuals, such as anal cancer, anal squamous intraepithelial lesions $[15,16]$ and reduce HIV risk $[5,6]$. Moreover, the rectum might act as a reservoir and thereby play a major role in repeat positive urogenital infections [4].

In the control of anorectal chlamydia there are 2 key stones: first is identification (diagnosis by testingl and second is treatment. In high risk groups there is evidence that many anorectal STI are missed by current testing practice on indication. A study using routine universal anorectal testing in high-risk women found that selective testing on indication misses over half of anorectal infections ( $48 \% \mathrm{Ct}, 80 \% \mathrm{Ng}$ ) [17]. It is unknown whether selective testing on indication misses infections in the general female population, due to lack of studies in this population.

Adequate treatment for anorectal chlamydia is currently under debate. Guidelines in the UK and US recommend both single-dose azithromycin and a 7-day course of doxycycline as equal treatments for uncomplicated anorectal chlamydia in non pregnant women [12]. In the Netherlands, doxycycline is recommended for anorectal chlamydia [13]. Several studies have reported substantial microbial failure rates of up to $40 \%$ for single-dose azithromycin (1.0 g) used against anorectal chlamydia [18-22], or suggest that doxycycline may be more effective than azithromycin in the treatment of rectal chlamydial infections [23]. Anorectal control strategies (treatment and testing) are thereby in need of critical reflection. 
To inform optimal control strategies for anorectal STIs in women, first the prevalence of anorectal STI was determined by using routine universal collected data in the general STI clinic population of women. Such data is scarce, because of the general lack of a routine universal screening practice in women in STI control settings. Moreover, little is known about alternative transmission routes such as the anal use of fingers and/or sex toys. By assessing associations with medical and behavioural history, we aim to estimate the number of anorectal infections missed by the current practice of selective testing on indication and to formulate recommendations for control i.e., the testing and treatment strategies.

\section{Methods}

\section{Study population}

The outpatient STI clinic of the South Limburg Public Health Service provides about 6000 consultations annually, offering free examination and treatment at three regional outpatient STI clinics. Between May 2012 and July 2013, three consultation nurses (out of 13) offered all their female patients aged 18 years and older $(n=663)$ routine testing for urogenital and anorectal chlamydia and gonorrhoea. This yielded a total of 654 consultations by 611 women for analysis (participation 92.2\%). Fifty-two (7.8\%) women declined an anorectal swab; reported reasons for non-participation were inconvenience (65\%), fear (19\%) and lack of necessity (16\%). Non-participants were slightly younger than participants (median 21 years versus 23 years, $P<0.001$ ). Urogenital chlamydia prevalence was similar for non-participants and participants $113.5 \%$; (7/52) versus $11.2 \%$ (73/654), $P=0.60)$. Gonorrhoea infections were not observed in both groups. The study was approved by the Medical Ethics Committee of Maastricht University (11-4-108).

\section{Study procedures and definitions}

Women provided self-collected vaginal swabs and self-collected anorectal swabs, which studies have proven to be a generally acceptable, valid and feasible approach $[3,24,25]$. Trained study nurses provided women with a visual diagram and oral instructions about how to take separate self-collected vaginal and rectal swabs. For the vaginal swab, the patient was instructed to insert the swab $2.5 \mathrm{~cm}$ into the vagina, rotate it for 5 to 10 seconds, and then place it in a capped tube to avoid potential contamination. This procedure was repeated in the anus for the rectal swab. Samples were tested for Chlamydia trachomatis and Neisseria gonorrhoeae using nucleic acid amplification assays according to the manufacturer's procedure (polymerase chain reaction [PCR; Roche Cobas 4800, San Francisco, CA]). Serum was tested for Treponema pallidum hemagglutination (TPHA) and HIV; all the women were TPHA and HIV negative. Each consult also included a standardised medical and sexual history taken by trained study nurses. It asked about self-reported symptoms and sexual behaviour in the past six months, i.e., 'Did you 
practise anal sex in the past six months?'. Anal symptoms included rectal discharge, bleeding, pain, redness, burning sensation, or itching. Swingers were defined as women who were part of a male-female couple that had sex with other male-female couples and their self-identified heterosexual sex partners. Prostitutes were defined as women who reported having had sex for money in the past six months. Women who were prostitute and/or swinger were defined as prostitutes/swingers. All data was registered in an electronic patient registry.

\section{Statistical analysis}

Women were assigned to one of three non-overlapping hierarchically constructed indication categories based on reported behaviour and symptoms. Women in the "indication" category reported at least anal symptoms and/or anal sex, whether or not in combination with anal use of fingers and/or toys. Women who were assigned to the "fingers/toys" category only reported the anal use of fingers and/or toys and reported no anal symptoms and no anal sex. Women who reported no anal symptoms, no anal sex, and no anal use of fingers or toys were assigned to the "without indication" category. As no gonorrhoea infections were observed, statistical analyses focussed on chlamydia only. The prevalence of chlamydia was calculated by dividing the number of positive tests by the total number of tests, multiplied by 100 . Univariate and multivariate logistic regression were used to identify determinants independently associated with anorectal chlamydia. Determinants tested were indication (with indication versus the two other categories combined), age categories, prostitutes/swingers (prostitutes and swingers versus other women), and use of fingers/toys (versus no use of fingers/toys). Anorectal infections in the categories "without indication" and "fingers/toys" were defined as infections missed by selective testing on indication as in current care. The share of infections missed was compared between indication categories, age categories (reference $\geq 29$ years) and prostitutes/swingers using univariate and multivariate logistic regression. Interactions terms were added between indication categories, age categories and prostitutes/swingers in the multivariate models, but none were statistically significant and were removed from in the final models.

Finally, to assess the anatomic site distributions of urogenital and anorectal chlamydia, all women who tested positive for chlamydia were assigned to a non-overlapping distribution category: (1) urogenital only, (2) urogenital and anorectal, or (3) anorectal only. Restricting to chlamydia positives, this variable was compared over indication categories (with indication versus the two other categories combined), age categories, and prostitutes/swingers using Fisher's exact test. A P value of $<0.05$ was considered statistically significant. Analyses were performed using SPSS version 17.0.0 (IBM Inc., Somers, NY, USA). Written informed consent for participation in the study was obtained from participants. Written informed consent was not obtained from a parent or guardian. 


\section{Results}

We included 654 consultations with an overall median participant age of 23 years (inter-quartile range: 21 to 34). The overall prevalence was 11.2\% (73/654) for urogenital chlamydia and $8.4 \%$ (55/654) for anorectal chlamydia. Gonorrhoea infections were not observed. Overall, anal use of fingers was reported by $20.3 \%$ (133/654) of the women, anal use of toys by $8.9 \%$ (58/654), anal sex with a steady partner by $24.0 \%$ (157/654), anal sex with a casual partner by $13.1 \%(86 / 654)$ and anal symptoms by $3.1 \%(20 / 654)$. Anal symptoms reported were itching $(n=7)$, ulceration $(n=3)$, redness $(n=2)$, discharge $(n=1)$, pain/burning sensation $(n=5)$, bleeding $(n=3)$ and unspecified ( $n=2)$. Only 3 women reported a combination of (two) symptoms.

\section{Indication categories}

In total, $31.0 \%(203 / 654)$ of the women were assigned to the "indication" category (i.e. they reported anal sex and/or symptoms), 7.3\% (48/654) to the "fingers/toys" category (reported anal use of fingers/toys only), and $61.6 \%(403 / 654)$ to the "without indication" category (no reported anal symptoms, no anal sex, and no anal use of fingers/toys). Women without indication were younger than women with indication (median of 22 years versus 36 years). The share of prostitutes/swingers was higher in women using fingers/toys only compared to women without indication (table 1).

\section{Chlamydia prevalence and associated determinants}

Prevalence of anorectal chlamydia was $7.9 \%$ (16/203) for women with indication and $8.6 \%$ (39/451) for the other women (categories without indication and fingers/toys ) ( $P=0.74$ ). Prevalence in the three indication categories is displayed in table 1. Young age was the only determinant associated with anorectal chlamydia ( $\leq 21$ years $14.2 \%$ lodds ratio 3.79 (1.75-8.20)), $22-28$ years $7.0 \%$ lodds ratio $1.73(0.75-4.00)$ and $\geq 29$ years $4.2 \%$ ). Being prostitute/swinger was not associated with anorectal chlamydia; prevalence was $3.0 \%(5 / 168)$ for prostitutes/swingers versus $10.3 \%(50 / 486)$ for other women ( $P=0.13)$. In total, 136 women reported to have used fingers or toys, whether or not in combination with anal sex. Prevalence in those women was $5.1 \%(7 / 136)$ versus $9.3 \%(48 / 518)$ in women who did not report to have used fingers or toys $(\mathrm{P}=0.82)$.

\section{Missed infections by selective testing on indication}

In total, 55 anorectal chlamydia infections were diagnosed; 67.3\% (37/55) were diagnosed in women without indication (table 1). Only 2/55 anorectal infections were diagnosed in the fingers/ toys category. Combining fingers/toys with the without indication category las is usually the case in current care), the proportion missed by current care that uses selective testing was $70.9 \%$ 
Table 1: Characteristics of women attending the STI clinic routinely universally screened for urogenital and anorectal chlamydia

\begin{tabular}{|c|c|c|c|c|}
\hline & Indication $^{a}$ & $\begin{array}{l}\text { Fingers/toys } \\
\text { only }\end{array}$ & $\begin{array}{l}\text { Without } \\
\text { indication }\end{array}$ & Total \\
\hline & $\begin{array}{l}N=203 \\
\%(n)\end{array}$ & $\begin{array}{l}N=48 \\
\%(n)\end{array}$ & $\begin{array}{l}N=403 \\
\%(n)\end{array}$ & $\begin{array}{l}N=654 \\
\%(n)\end{array}$ \\
\hline \multicolumn{5}{|l|}{ Age } \\
\hline$\leq 21$ years & $19.2(39)^{* *}$ & $12.5(6)^{* *}$ & $41.2(166)$ & $32.3(211)$ \\
\hline $22-28$ years & $27.1(55)$ & $25.0(12)$ & $40.0(161)$ & 34.9 (228) \\
\hline$\geq 29$ years & $53.7(109)$ & $62.5(30)$ & $18.9(76)$ & $32.9(215)$ \\
\hline Prostitutes/swingers & $39.4(80)^{*}$ & $66.7(32)^{*}$ & $13.9(56)$ & 25.7 (168) \\
\hline \multicolumn{5}{|l|}{ Chlamydia prevalence } \\
\hline Urogenital & $9.4(19)$ & $4.2(2)$ & $12.9(52)$ & $11.2(73)$ \\
\hline Anorectal & $7.9(16)$ & $4.2(2)$ & $9.2(37)$ & $8.4(55)$ \\
\hline $\begin{array}{l}\text { Anatomic site distribution } \\
\text { chlamydia positives }\end{array}$ & $N=21$ & $\mathrm{~N}=2$ & $N=53$ & $N=76$ \\
\hline Urogenital only & $23.8(5)$ & $0(0)$ & $30.2(16)$ & $27.6(21)$ \\
\hline Anorectal only & $9.5(2)$ & $0(0)$ & $1.9(1)$ & $3.9(3)$ \\
\hline Urogenital and anorectal & $66.7(14)$ & $100(2)$ & $67.9(36)$ & $68.4(52)$ \\
\hline
\end{tabular}

* $P<0.0001$ compared to without indication by Chi-square test.

** $P<0.0001$ compared to without indication and age 29 years or older by Chi-square test.

a Women with indication reported anal use of fingers and/or toys in $43.3 \%$ of consultations, symptoms in $9.9 \%$, anal sex with a steady partner in $77.3 \%$, and anal sex with a casual partner in $42.4 \%$.

(39/55). No determinants were found to be associated with missed anorectal infections. For example the proportion missed was $60.0 \%$ in prostitutes/swingers versus $72.0 \%$ in other women $(P=0.93)$. The proportion missed was $70.0 \%$ in age $\leq 21$ years, $81.3 \%$ in age $22-28$ years and $55.6 \%$ in age $\geq 29$ years $(P=0.47)$.

\section{Anatomic site distribution}

Of all urogenital and anorectal chlamydia infections found, 68.4\% (52/76) were concurrent urogenital and anorectal infections. Of all chlamydia infections, only three infections were isolated anorectal $(3.9 \%, 3 / 76)$ : two in women with indication and one in a woman without indication (figure 1). Of the 73 women with urogenital chlamydia, 71.2\% (52/73) also had an anorectal chlamydia infection. Of the 55 women with anorectal chlamydia, 94.5\% (52/55) also had a urogenital chlamydia infection. The anatomic site distribution of chlamydia infections was not associated with indication categories $(P=0.31)$, age $(P=0.90)$, or prostitutes/swingers $(P=0.27)$ (table 1). 
Figure 1: Anatomic site distribution of chlamydia in women attending the STI clinic routinely screened for urogenital and anorectal chlamydia

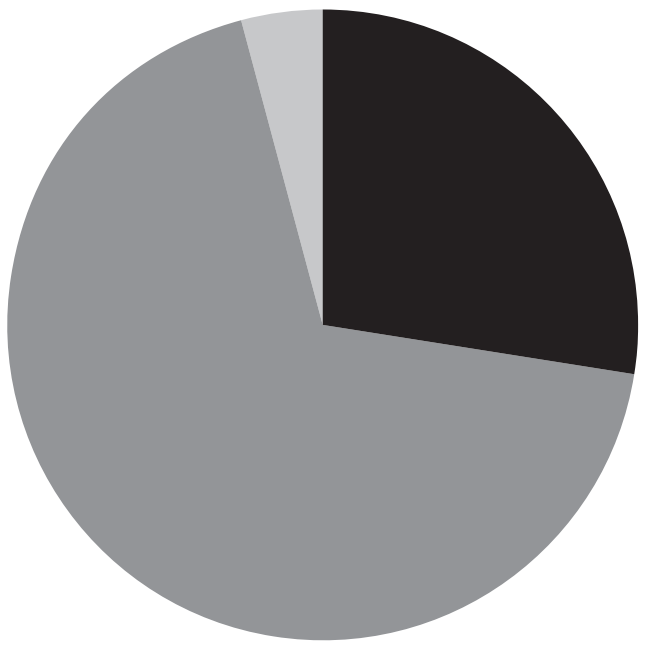

Urogenital only

$(27.6 \%, n=21)$

Urogenital and anorectal

$(68.4 \%, n=52)$

Anorectal only

$(3.9 \%, n=3)$

\section{Discussion}

This study revealed alarmingly high numbers of anorectal chlamydia in women. One in 12 women and even one in 7 young women was diagnosed with anorectal chlamydia. As over two thirds of these infections are currently being missed, current selective testing on indication of symptoms and anal sexual history is not an appropriate control strategy for anorectal chlamydia in women visiting an STI clinic. Almost all women with anorectal chlamydia had concurrent urogenital chlamydia (95\%). To our knowledge, this is the first study with routine universal anorectal testing, i.e. independent of reported behaviour, symptoms or urogenital positivity, in a general group of women who visited an STI clinic and who took different sexual risks, including anal use of fingers and toys.

Indication (anal sex or symptoms) was not associated with anorectal chlamydia. Yet current guidelines for anorectal STIs advocate selective symptom- and sexual history based testing for women $[12,13]$. Including the anal use of fingers/toys in this testing on indication would only reveal a small number of missed infections (3.6\%), as these practices are common but rarely practiced without anal sex (7\%). Moreover, use of fingers/toys was not associated with anorectal chlamydia. This suggests that anal use of fingers/toys, as well as report of anal sex or symptoms are not useful indicators to guide testing for anorectal chlamydia. 
The prevalence of anorectal chlamydia was substantial (8\%) (55/654). Young age ( $\leq 21$ years) was found to be associated with anorectal chlamydia showing a strikingly high $14 \%$ prevalence in this group of women, consistent with studies in high risk women visiting an STI clinic [5,6,9]. Thereby the absolute number of anorectal infections that are currently missed in the general female STI clinic population is likely substantial, especially in young women. This is consistent with earlier findings in high-risk groups at our clinic, such as female swingers (chlamydia 48\%, gonorrhoea $80 \%$ ) and men who have sex with men (MSM) (chlamydia 43-55\%, gonorrhoea 29-100\%) [17]. More data is needed from other settings to confirm our observations by routine anorectal testing.

In contrast to MSM, anorectal chlamydia in women was rarely isolated. In current study, one woman had an isolated anorectal infection but did not report anal sex or symptoms. Possible explanations for this could be underreporting, a false negative urogenital test $[4,8]$, or autoinoculation from a spontaneously cleared urethral/vaginal infection [4,5]. The large percentage of concurrent urogenital and anorectal chlamydia infections in women was therefore notable: $95 \%$ of women with anorectal chlamydia also had urogenital chlamydia, and $71 \%$ of women with urogenital chlamydia also had anorectal chlamydia. Previous studies without routine universal testing in women also reported large shares of concurrent infections (36-90\%) [2,4-10,26]. It is not clear what causes these concurrent infections, although possible explanations could be underreporting of anal sex, autoinoculation with vaginal secretions $[4,5,8,9,26]$ or concurrent transmission during sex. Majority (71\%) of anorectal chlamydia positives did not report anal sex or symptoms. Autoinoculation from the vagina to the rectum therefore seems possible. We hypothesize that autoinoculation could also occur from the rectum to the vagina. Even in the absence of sexual activity, the gastro intestinal tract could provide a constant source of organisms which may reinfect the genital tract [31]. Such (repeat) urogenital infections could lead to reproductive tract morbidity [6]. Further study on this subject is needed, for example by including anorectal chlamydia in mathematical models and by bacterial load studies, the clinical and public health impact of anorectal chlamydia in women could be explored further.

Nevertheless, state of the art practice in chlamydia control entails the use of highly sensitive NAATs to test for chlamydia. Although NAATs are not yet FDA proved for anorectal testing, their use is highly recommended, accepted, and part of standard operating procedures in many care settings [14]. A positive NAAT, i.e., diagnosed anorectal chlamydia, is in practice followed by antibiotic treatment. In MSM, an anorectal swab positive for chlamydia is considered an infection, and is treated with antibiotics to prevent transmission to the population and complications in individuals. To overcome current insufficient case management of anorectal infections in women, testing and treatment strategies need to be improved, to better identify and treat infections. Study participation was high (93\%), suggesting a high feasibility and acceptability of anorectal testing in women who do not have an indication. Therefore, routine universal anorectal 
screening could be an option, although this will increase costs substantially. No studies have evaluated cost effectiveness of anorectal screening for chlamydia/gonorrhoea in women. However, in MSM, anorectal screening (when prevalence > 2.69\% (IQR, 1.68-3.71\%)) can be a cost-effective intervention to reduce HIV infection [28-30].

When a more restricted policy is preferred, anorectal testing only in women with urogenital infection or direct treatment effective for both urogenital and non-urogenital chlamydia would detect and treat $95 \%$ of anorectal infections, since 52 of 55 anorectal infections had co-occurrence of urogenital chlamydia. However, for the former option delay between urogenital and anorectal tests and subsequent treatments could be a problem in practice. The substantial anorectal chlamydia prevalence and high co-occurrence with urogenital chlamydia fuels the need for debate on what is adequate treatment for anorectal chlamydia [18-21,23]. The currently used treatment regimes for uncomplicated anorectal chlamydia both have drawbacks; higher treatment failure rates are reported for azithromycin [19-21,23] and compliance for doxycycline could possibly be an issue in practice [27]. More research, for example a randomised controlled trial of azithromycin versus doxycycline, including compliance, is needed to formulate treatment recommendations.

Several study limitations need to be acknowledged. We only included women who visited the STI clinic, so our results might not fully represent those that could be found in the general female population or within other healthcare settings. Although our instructions on specimen collection were clear, we cannot entirely rule out the possibility of specimen contamination (for example via the urogenital-anorectal route). As women attending the STI clinic were randomly assigned to a consultation nurse, possible selection bias is likely minimal. Data on other high risk sexual behaviors (i.e., number of partners, new or concurrent partnerships, substance use, condom use) were not available, and their association with anorectal chlamydia in women could not be assessed. In our study, the prevalence or the proportion of infections missed by current selective testing in the non-participants is unknown. Eligible non-participants were slightly younger than participants. A study by Sethupathi et al. found women most at risk for anorectal infections included women aged $<20$ years as was also found in current study. Therefore, the prevalence of anorectal infections may be underestimated in current study, yet due to the high response (93\%), bias is expected to be minimal. 


\section{Conclusions}

In conclusion, prevalence of anorectal chlamydia in women was high and current selective testing on indication is not an appropriate control strategy to identify and treat anorectal chlamydia infections. Almost all women with anorectal chlamydia had concurrent urogenital chlamydia. More research is needed on the public health and clinical implications of anorectal chlamydia in women.

\section{Acknowledgements}

The authors acknowledge the study participants and contributions of the nurses and doctor's assistants of the STI clinic, especially Eslie Bos, Renee Keesmekers, Gertrudie Maas, Chris Martijn, Sandra Meissner and Eveline van der Veen. The authors also acknowledge Marianne van der Sande PhD from the National Institute for Public Health and the Environment (RIVM) for her valuable contribution to the study. 


\section{References}

1. Reported STD's in the United States. 2012 National Data for Chlamydia, Gonorrhea and Syphilis. http://www.cdc.gov/nchhstp/ newsroom/docs/STD-Trends-508.pdf.

2. Bax CJ, Quint KD, Peters RP, Ouburg $S$, Oostvogel PM, Mutsaers JA, Dorr PJ, Schmidt S, Jansen C, van Leeuwen AP, Quint WGV, Trimbos JB, Meijer CJLM, Morre SA: Analyses of multiple-site and concurrent Chlamydia trachomatis serovar infections, and serovar tissue tropism for urogenital versus rectal specimens in male and female patients. Sex Transm Infect 2011, 87(6):503-507.

3. Schoeman SA, Stewart CM, Booth RA, Smith SD, Wilcox MH, Wilson JD: Assessment of best single sample for finding chlamydia in women with and without symptoms: a diagnostic test study. BMJ 2012, 345:e8013.

4. Barry PM, Kent CK, Philip SS, Klausner JD: Results of a program to test women for rectal chlamydia and gonorrhea. Obstet Gynecol 2010, 115(4):753-759.

5. Hunte T, Alcaide M, Castro J: Rectal infections with chlamydia and gonorrhoea in women attending a multiethnic sexually transmitted diseases urban clinic. Int J STD AIDS 2010, 21(12):819-822.

6. Javanbakht M, Gorbach P, Stirland A, Chien M, Kerndt P, Guerry S: Prevalence and correlates of rectal Chlamydia and gonorrhea among female clients at sexually transmitted disease clinics. Sex Transm Dis 2012, 39(12):917-922.

7. Koedijk FD, van Bergen JE, Dukers-Muijrers $\mathrm{NH}$, van Leeuwen AP, Hoebe CJ, van der Sande MA: The value of testing multiple anatomic sites for gonorrhoea and chlamydia in sexually transmitted infection centres in the Netherlands, 2006-2010. Int J STD AIDS 2012, 23(9):626-631.

8. Peters RP, Nijsten N, Mutsaers J, Jansen CL, Morre SA, van Leeuwen AP: Screening of oropharynx and anorectum increases prevalence of Chlamydia trachomatis and Neisseria gonorrhoeae infection in female STD clinic visitors. Sex Transm Dis 2011, 38(9):783-787.

9. Sethupathi M, Blackwell A, Davies H: Rectal Chlamydia trachomatis infection in women. Is it overlooked? Int J STD AIDS 2010, 21(2):93-95.

10. Shaw SG, Hassan-Ibrahim M, Soni S: Are we missing pharyngeal and rectal infections in women by not testing those who report oral and anal sex? Sex Transm Infect 2013, 89(5):397.

11. Ivens D, Macdonald K, Bansi L, Nori A: Screening for rectal chlamydia infection in a genitourinary medicine clinic. Int J STD AIDS 2007, 18(6):404-406.

12. Workowski KA, Berman SM: Sexually transmitted diseases treatment guidelines, 2006. MMWR Recomm Rep 2006, 55(RR-11):1-94.

13. Venereologie NVD: Diagnostiek en behandeling van seksueel overdraagbare aandoeningen (SOA) korte samenvatting SOA richtlijn. http:// www.huidziekten.nl/richtlijnen/SOA-richtlijnsamenvatting-2011.pdf.

14. Schachter J, Philip SS: Testing men who have sex with men for urethral infection with Chlamydia trachomatis and Neisseria gonorrhoeae is only half the job, and we need the right tools. Sex Transm Dis 2011, 38(10):925-927. 
15. Holly EA, Ralston ML, Darragh TM, Greenblatt RM, Jay N, Palefsky JM: Prevalence and risk factors for anal squamous intraepithelial lesions in women. J Natl Cancer Inst 2001, 93(11):843-849.

16. Moscicki AB, Hills NK, Shiboski S, Darragh TM, Jay N, Powell K, Hanson E, Miller SB, Farhat S, Palefsky J: Risk factors for abnormal anal cytology in young heterosexual women. Cancer Epidemiol Biomarkers Prev 1999, 8(2):173-178.

17. van Liere GA, Hoebe CJ, Niekamp AM, Koedijk FD, Dukers-Muijrers NH: Standard symptomand sexual history-based testing misses anorectal Chlamydia trachomatis and neisseria gonorrhoeae infections in swingers and men who have sex with men. Sex Transm Dis 2013, 40(4):285-289.

18. Drummond F, Ryder N, Wand H, Guy R, Read P, McNulty AM, Wray L, Donovan B: Isazithromycin adequate treatment for asymptomatic rectal chlamydia? Int J STD AIDS 2011, 22(8):478-480.

19. Elgalib A, Alexander S, Tong CY, White JA: Seven days of doxycycline is an effective treatment for asymptomatic rectal Chlamydia trachomatis infection. Int J STD AIDS 2011, 22(8):474-477.

20. Hathorn E, Opie C, Goold P: What is the appropriate treatment for the management of rectal Chlamydia trachomatis in men and women? Sex Transm Infect 2012, 88(5):352-354.

21. Steedman NM, McMillan A: Treatment of asymptomatic rectal Chlamydia trachomatis: is single-dose azithromycin effective? Int J STD AIDS 2009, 20(1):16-18.
22. Dukers-Muijrers NH, Speksnijder AG, Morre SA, Wolffs PF, van der Sande MA, Brink AA, van den Broek IV, Werner MI, Hoebe CJ: Detection of anorectal and cervicovaginal Chlamydia trachomatis infections following azithromycin treatment: prospective cohort study with multiple time-sequential measures of rRNA, DNA, quantitative load and symptoms. PLoS One 2013, 8(11):e81236.

23. Khosropour CM, Dombrowski JC, Barbee LA, Manhart LE, Golden MR: Comparing azithromycin and doxycycline for the treatment of rectal chlamydial infection: a retrospective cohort study. Sex Transm Dis 2014, 41(2):79-85.

24. Hoebe CJ, Rademaker CW, Brouwers EE, ter Waarbeek HL, van Bergen JE: Acceptability of self-taken vaginal swabs and first-catch urine samples for the diagnosis of urogenital Chlamydia trachomatis and Neisseria gonorrhoeae with an amplified DNA assay in young women attending a public health sexually transmitted disease clinic. Sex Transm Dis 2006, 33(8):491-495.

25. van der Helm JJ, Hoebe CJ, van Rooijen MS, Brouwers EE, Fennema HS, Thiesbrummel HF, Dukers-Muijrers NH: High performance and acceptability of self-collected rectal swabs for diagnosis of Chlamydia trachomatis and Neisseria gonorrhoeae in men who have sex with men and women. Sex Transm Dis 2009, 36(8):493-497.

26. Ding A, Challenor R: Rectal Chlamydia in heterosexual women: more questions than answers. Int J STD AIDS 2013, 0(0):1-6.

27. Khosropour CM, Manhart LE, Colombara DV, Gillespie CW, Lowens MS, Totten PA, Golden 
MR, Simoni J: Suboptimal adherence to doxycycline and treatment outcomes among men with non-gonococcal urethritis: a prospective cohort study. Sex Transm Infect 2014, 90(1):3-7.

28. Chesson HW, Bernstein KT, Gift TL, Marcus JL, Pipkin S, Kent CK: The cost-effectiveness of screening men who have sex with men for rectal chlamydial and gonococcal infection to prevent HIV Infection. Sex Transm Dis 2013, 40(5):366-371.

29. Xiridou M, Vriend HJ, Lugner AK, Wallinga J, Fennema JS, Prins JM, Geerlings SE, Rijnders BJ, Prins M, de Vries HJ, Postma MJ, van Veen MG, Schim van der Loeff MF, van der Sande MA: Modelling the impact of chlamydia screening on the transmission of HIV among men who have sex with men. BMC Infect Dis 2013, 13:436.

30. Vriend HJ, Lugner AK, Xiridou M, van der Loeff MF S, Prins M, de Vries HJ, Geerlings SE, Prins JM, Rijnders BJ, van Veen MG, Fennema JS, Postma MJ, van der Sande MA: Sexually transmitted infections screening at HIV treatment centers for MSM can be cost-effective. AIDS 2013, 27(14):2281-2290.

31. Rank RG, Yeruva L: Hidden in plain sight: chlamydial gastrointestinal infection and its relevance to persistence in human genital infection. Infect Immun 2014, 82(4):1362-1371. 



\section{Chapter 7}

\section{Prevalence of and factors associated with rectal-only chlamydia and gonorrhoea in women and in men who have sex with men}

Geneviève AFS van Liere, Martijn S van Rooijen, Christian JPA Hoebe, Titia Heijman, Henry JC de Vries, Nicole HTM Dukers-Muijrers.

PLoS ONE 2015 oct 29; 10(10). doi:

10.1371/journal.pone.0140297 


\section{Abstract}

\section{Background}

Both anorectal Chlamydia trachomatis (CT) and Neisseria gonorrhoea (NG) can occur as a rectal-only infection or concurrently with simultaneous urogenital infection with the same pathogen. Characterising the target groups in which rectal-only infections occur may improve the efficacy of screening practices.

\section{Methods}

We analysed data from two Dutch outpatient sexually transmitted infection (STI) clinics between 2011 and 2012. We included all men who have sex with men (MSM) (n=9549) and women ( $\mathrm{n}=11113$ ), $\geq 18$ years, who had been tested for anorectal and urogenital CT and/or NG (either as a result of reporting anal sex/symptoms or via routine universal testing). Factors associated with rectal-only CT and NG infections were assessed using univariable and multivariable logistic regression.

\section{Results}

In MSM, anorectal CT prevalence was 9.8\% (693/7094), anorectal NG prevalence was $4.2 \%$ (397/9534). In women this was 9.5\% overall (439/4597) and 0.9\% (96/10972) respectively. Anorectal CT prevalence among women who were routinely universally tested was 10.4\% (20/192), for selective testing this was $9.5 \%(419 / 4405)(p=0.68)$. Anorectal NG infections were not detected among women who were routinely universally tested $(\mathrm{p}=0.19)$. Among CT or NG positive MSM, rectal-only CT infections were found in 85.9\% (595/693), for NG this was 85.6\% (340/397) respectively. In positive women these figures were $22.1 \%$ (97/439) for CT and $20.8 \%$ (20/96) for NG, respectively. In MSM, independent factors associated with rectal-only CT were: being a sex worker (ORO.4,CI0.2-1.0), exclusively having sex with men (OR3.4,Cl1.7-6.8), and absence of urogenital symptoms (OR0.2,CI0.2-0.4). In women, these factors were: older age (OR2.3, CI1. 3-4.0) and non-Western nationality (OR1.8, CI1.0-3.5). Factors associated with rectal-only NG in MSM were: having been warned for STIs by an (ex) partner (OR2.9,Cl1.1-7.5), oropharyngeal NG infection (OR2.4,CI1.0-5.3), and absence of urogenital symptoms (OR0.02,Cl0.01-0.04), while in women no significant factors were identified.

\section{Discussion}

The prevalence of anorectal CT and NG was substantial in MSM and prevalence of anorectal CT was also substantial in women. Anorectal infections occurred mostly as rectal-only infections in MSM and mostly concurrent with other infections in women. Given the lack of useful indicators for rectal-only infections, selective screening based on a priori patient characteristics will have low discriminatory power both in relation to MSM and women. 


\section{Introduction}

With the introduction of sensitive nucleic acid amplification assays (NAAT) tests, anorectal testing for Chlamydia trachomatis (CT) and Neisseria gonorrhoea (NG) has become more commonplace in sexually transmitted infection (STI) clinics[1,2]. Anorectal CT and NG are common in men who have sex with men (MSM) and in women[3]. Anorectal testing is important since the majority of infections are asymptomatic. Undetected infections could lead to further spread of infection in the population and the development of sequelae within individuals. Moreover, anorectal CT and NG infections facilitate HIV transmission[4].

There is a lack of international consensus regarding the adequate treatment for anorectal CT. Guidelines in the US recommend single-dose azithromycin or a 7-day course of doxycycline as equally effective treatments for uncomplicated anorectal CT in MSM and non-pregnant women[5]. In the Netherlands and the UK, doxycycline is recommended for anorectal CT[6,7].

Prevalence of anorectal CT has been reported to be as high as $24.4 \%[3,8-17]$ among MSM and $17.5 \%$ among women[3,13-15,18-25], with rates for NG as high as $17.9 \%[3,8-11,13-15,17]$ and $13.4 \%[3,11,13-15,18,19,22-25]$ for MSM and women respectively. Insight into the factors associated with anorectal infections, including demographic and behavioural factors, can facilitate the identification of high-risk groups and inform guidelines on anorectal testing. Previous studies in MSM and women attending STI clinics have shown that being of a younger age[9,11] and having multiple sex partners[8,11] are both associated with having anorectal CT and NG infections. Notably, anal sex has not been found to be associated with anorectal CT, but has been associated with anorectal NG[13,14].

Anorectal infections can be rectal-only infections, i.e., infection at the anorectal site only, or can occur concurrently with simultaneous urogenital and/or oropharyngeal infections with the same pathogen. Previous studies in MSM have reported a high proportion of rectal-only CT (up to 90\%) and NG infections (up to 70\%)[8-11]. In contrast, in women, a relatively low proportion of rectalonly CT and NG infections have been reported (between $0 \%$ to $44 \%$ )[18,19,22-27]. It is unknown which factors determine such differences in the relative proportions of rectal-only infections. These differences may be related to for example sexual risk behaviour or anatomical differences, but are probably not caused by the characteristics of the CT strain[28]. If a single dose of azithromycin is effective in treating anorectal CT, concurrent urogenital and anorectal CT infections would also be opportunistically treated[13], as is the case with concurrent NG infections. However, rectal-only CT and NG infections would not be opportunistically treated with an urogenital infection. Treatment of rectal-only infections depends on screening algorithms, as they are not routinely universally tested for in practice. Insight into the proportion of rectal-only infections and the factors associated with them could help to define individuals at risk for such untreated 
anorectal infection. This is important for the design of cost-effective screening guidelines specifying who should be tested at which anatomical sites.

By evaluating 20.662 unique STI clinic attendees tested at both urogenital and anorectal sites, this study aims to increase understanding of anorectal infections by assessing the prevalence of and factors associated with (rectal-only) anorectal CT and NG in MSM and in women.

\section{Methods}

\section{Study population}

This study was approved by the Medical Ethical Committee of the University of Maastricht (METC 11-4-108), who waived the need for consent to be collected from participants. Since retrospective data originated from standard care (in which one can opt-out for the use of their data for scientific research, as approved by METC 11-4-108) and were analysed anonymously, no further informed consent for data analysis was obtained. The outpatient Public Health Service STI clinics in Amsterdam (approximately 36.000 consultations in 2012) and South Limburg lapproximately 6500 consultations in 2012) offer free and anonymous STI testing to high-risk individuals. From January 2011 to December 2012, data from all MSM and women who had been tested for both urogenital and anorectal CT and/or NG were included once, from their most recent consultation ( $\mathrm{N}=20.662$ unique individuals, 9549 MSM and 11113 women) (figure 1). MSM were defined as men who had sex with men in the past 6 months.

\section{Study procedures}

All participants were routinely tested for urogenital CT and NG infections via a urine or urethral sample (men) or a vaginal or cervical swab (women).

In Amsterdam, if receptive anal sex was reported in the past 6 months, both MSM and women were screened for anorectal CT and NG infections. Irrespective of reported anal sex, MSM and high-risk women were routinely screened for anorectal NG infection. High-risk women who report active oral sex in the previous 6 months (regardless of condom use) and all MSM (irrespective of reported receptive oral sex) were routinely tested for oropharyngeal CT and NG. High-risk women were defined as: women who report symptoms, women who are commercial sex workers (CSW), women who have been warned for STIs by an (ex-) partner, or women who have been referred by another healthcare provider.

In South Limburg, for study purposes, between May and December 2012 women attending one of the 3 (out of 13) study nurses were routinely universally screened for anorectal CT and NG; this comprised 16\% (192/1200) of women tested at anorectal site in South Limburg[20]. Before and after this period, women were only tested at the anorectal site if they reported having anal 
sex or having anal symptoms. All MSM were routinely screened for anorectal CT and NG, irrespective of reported behaviour.

If receptive oral sex was reported to have taken place in the past 6 months, swingers and CSW were tested for oropharyngeal CT and NG infections. Other women were tested if there was a risk for an oropharyngeal-only infection, that is, without concurrent urogenital or anorectal infection, in addition to reported receptive oral sex. MSM were routinely universally screened for oropharyngeal CT and NG infections.

Each consultation included a standardised medical and sexual history taken by nurses, including self-reported symptoms and sexual behaviour over the past six months. Specimens tested consisted of vaginal/cervical swabs or urine, anorectal swabs and oropharyngeal swabs, either self-collected or collected by the nurse. All tests were performed according to the manufacturer's protocol. In the South Limburg clinic, specimens were processed at two regional laboratories using three different NAATs (SDA, Becton Dickinson ProbeTec ET system, Maryland, USA; Cobas Amplicor, Roche, California, USA; Cobas 4800, Roche, California, USA). In the Amsterdam clinic, the Aptima combo CT/NG assay for the detection of $\mathrm{C}$. trachomatis and $\mathrm{N}$. gonorrhoeae rRNA (Hologic Gen-Probe Inc., San Diego, USA) and the Aptima Ct assay were used. In Amsterdam, NG was routinely tested in MSM and high-risk women by culture. Serum was tested for Treponema pallidum haemagglutination (TPHA) (Bioelisa Syphilis 3.0 (Biokit, SA, USA)) and HIV lanti-HIV1/2, Axsym; Abbott Laboratories, Chicago, IL, USA). Reactive samples were confirmed using Western blot (South Limburg; HIVblot 2.2; Genelabs Diagnostics, Science Park, Singapore, Amsterdam; INNO-LIA HIV I/II Score; Fujirebio; 201 Great Valley Parkway, Malvern, USA).

\section{Statistical analysis}

The prevalence of anorectal CT and NG was calculated by dividing the number of positive tests by the total number of tests, multiplied by 100 . Anorectal CT prevalence is reported separately for women who were routinely universally tested and women who were tested based on the report of anal sex and/or symptoms (selective testing). Anorectal NG infections were reported overall, since anorectal NG was detected among women who were routinely universally tested $(p=0.19)$. Rectal-only infections were defined as an anorectal CT or NG infection without a concurrent urogenital infection with the same pathogen. Concurrent infections were defined as simultaneous urogenital and anorectal infection with the same pathogen. Oropharyngeal CT or NG infections were not taken into account in this classification, but assessed as a possible associated factor and presented in the tables. In all individuals, univariable and multivariable logistic regression were used to identify factors (independently) associated with (1) anorectal CT and anorectal NG and (2) among anorectal CT or NG positive individuals only, with rectal-only CT and NG infections compared to concurrent infections. Several variables were examined based on self-reports of behaviour during the 6 month period prior to consultation. The variables tested were: age, nationality, CSW, sexual preference (for MSM: exclusively having sex with men 
or not), number of sex partners, antibiotic use in the past 1-3 months, sexual practices lanal/ vaginal), condom use (anorectal/vaginal), intravenous (IV) drug use, having been warned for STIS by an (ex) partner (as in partner notification), symptoms (anorectal/urogenital), concurrent oropharyngeal CT, concurrent oropharyngeal NG, HIV status, TPHA positivity, and history of STI clinic consultations in the past 800 days. This last variable was divided into two variables: one for CT and one for NG. The CT variable was divided in the following mutually exclusive categories: (1) not tested, (2) previously tested CT negative, (3) previously had concurrent anorectal CT and (4) previously had at least one rectal-only CT. The NG variable was divided in the following mutually exclusive categories: (1) not tested, (2) previously tested NG negative, (3) previously had concurrent anorectal NG and (4) previously had at least one rectal-only NG. The variables age (for $M S M \leq 32,33-43, \geq 44$, and for women $\leq 22,23-27, \geq 28$ ), number of sex partners in the past 6 months $(\leq 2,3-5, \geq 6)$, and number of previous STI clinic consultations $(\leq 1,2-3, \geq 4)$, were all categorised into three groups based on tertile distributions. Urogenital symptoms were defined as: genital discharge, bleeding, itching, ulceration, swelling, pain, burning sensation and more frequent urination. Anal symptoms were defined as: anal discharge, bleeding, itching, ulceration, redness, swelling, pain and burning sensation. Anal sex was defined as insertive, receptive, or both. Anal condom use was categorised in the following way: 'no anal sex', 'always' and 'not always'. A similar variable was constructed for vaginal condom use. The factors anal sex and anal symptoms were excluded from multivariable analyses exploring associations with anorectal CT positivity in order to prevent bias by testing indication (selective testing based on report of anal sex/symptoms). Variables with $p<0.05$ were included with stepwise backward method in the multivariable model. In cases where variables were correlated (>0.6), the variable with the lowest $p$-value in univariable analyses was omitted from the multivariable model. All multivariable models were corrected for study site (Amsterdam/South Limburg). The results of the univariable analyses are included in the supplemental files. The results of the multivariable analyses are included in the manuscript. A p-value $<0.05$ was considered statistically significant. Analyses were performed using SPSS version 19.0 (IBM Inc., Somers, NY).

We visualised which factors would yield the highest number of infections if targeted in screening (for example if used as a screening indicator). Factors were selected based on their statistical significance in unadjusted univariable analyses and their usability as a screening tool, by including factors that were available from the patient at screening (for example, excluding any test result from that screening).

The proportion of anorectal CT and NG infections diagnosed, i.e. the number of diagnoses within a category divided by the total number of diagnoses multiplied by 100 , is presented in a bubble in order to visualise the yield of anorectal infections per factor. Thereby, the bubble represents the relative share in percentage of anorectal CT and NG infections detected. Each bubble represents an associated factor, and individuals can appear in multiple bubbles. The number of factors presented in one bubble plot was maximized to 8 , to ensure clarity of the figure. 
Figure 1 Flowchart of the study population

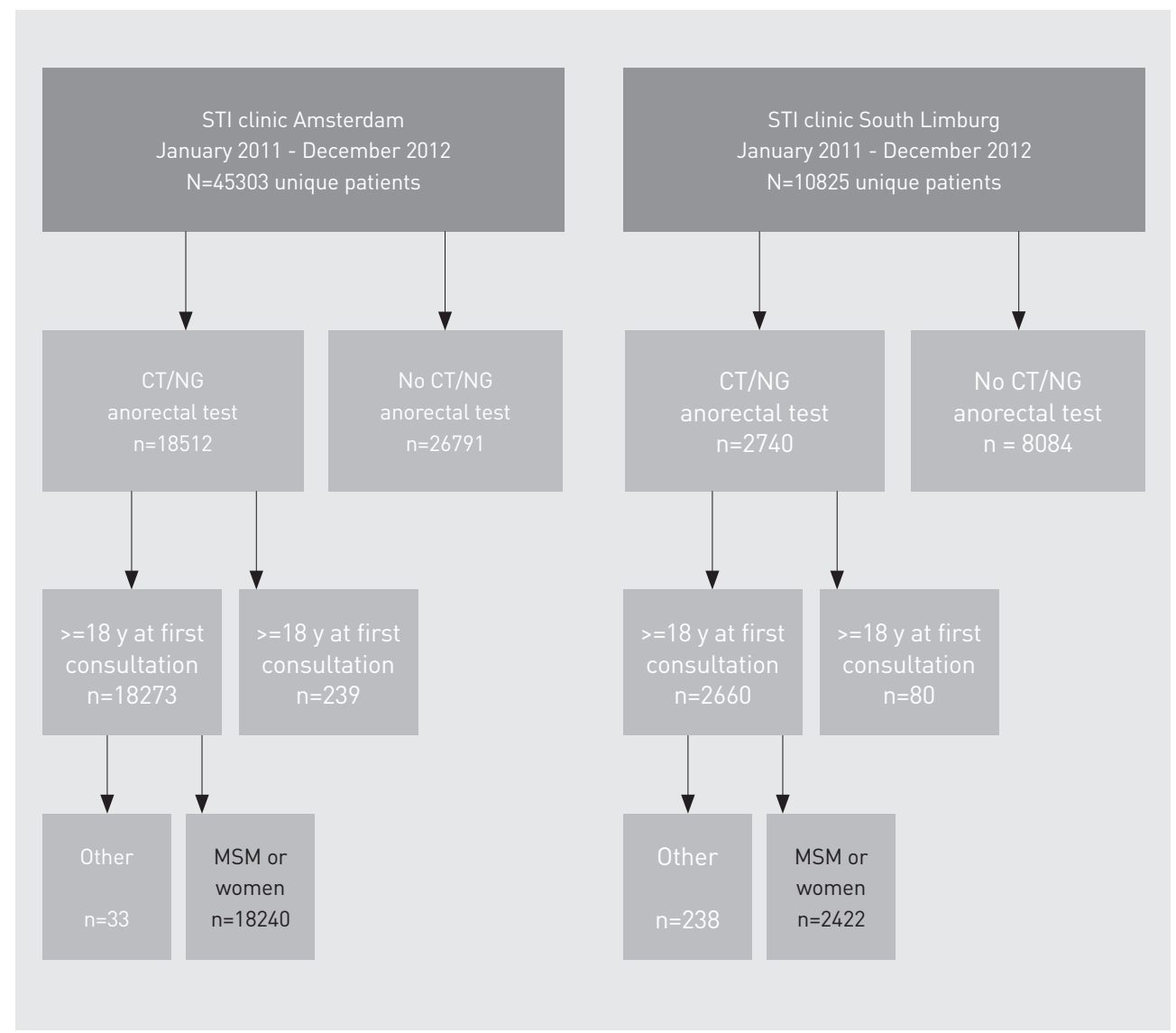

\section{Results}

In the study period, 45303 unique individuals vistited the STI clinic in Amsterdam, for South Limburg this was 10995 (figure 1).

In total, 20662 unique study participants who were tested for anorectal CT and/or NG (18240 Amsterdam, 2422 South Limburg) were included in our study; 9549 MSM and 11113 women. In total, $99.2 \%$ ( $n=20506$ ) were tested for $N G$ and $56.6 \%$ ( $n=11691)$ were tested for CT. The median age of MSM was 37 years (IQR 28-46), and the median age of women was 25 years (IQR 22-30). Anal sex was reported by $83.0 \%(n=7921)$ of MSM and by $33.3 \%(n=3696)$ of women, and anal symptoms were reported by $4.9 \%(n=449)$ of MSM and $1.9 \%(n=210)$ of women (table 1 and 2). 


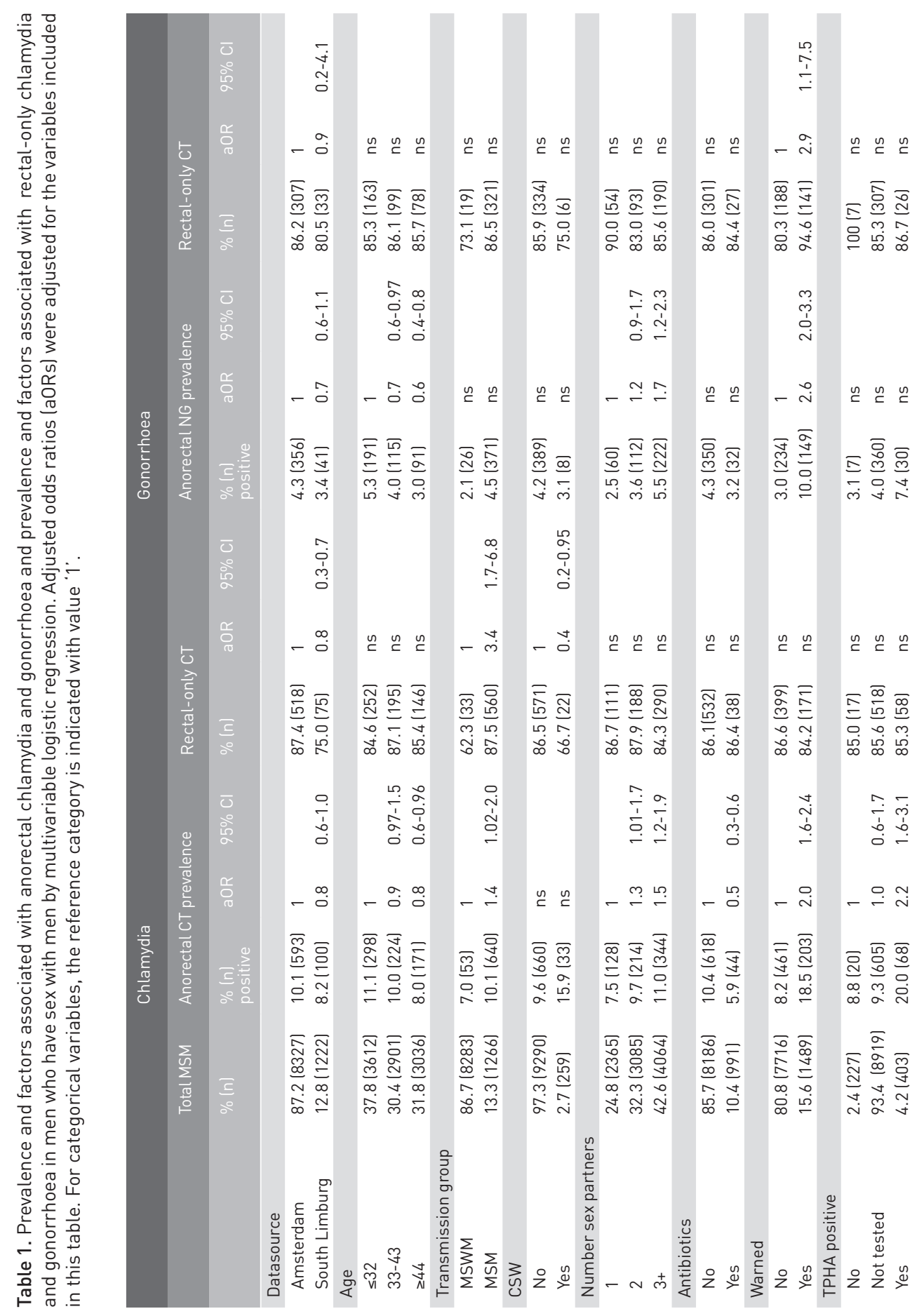




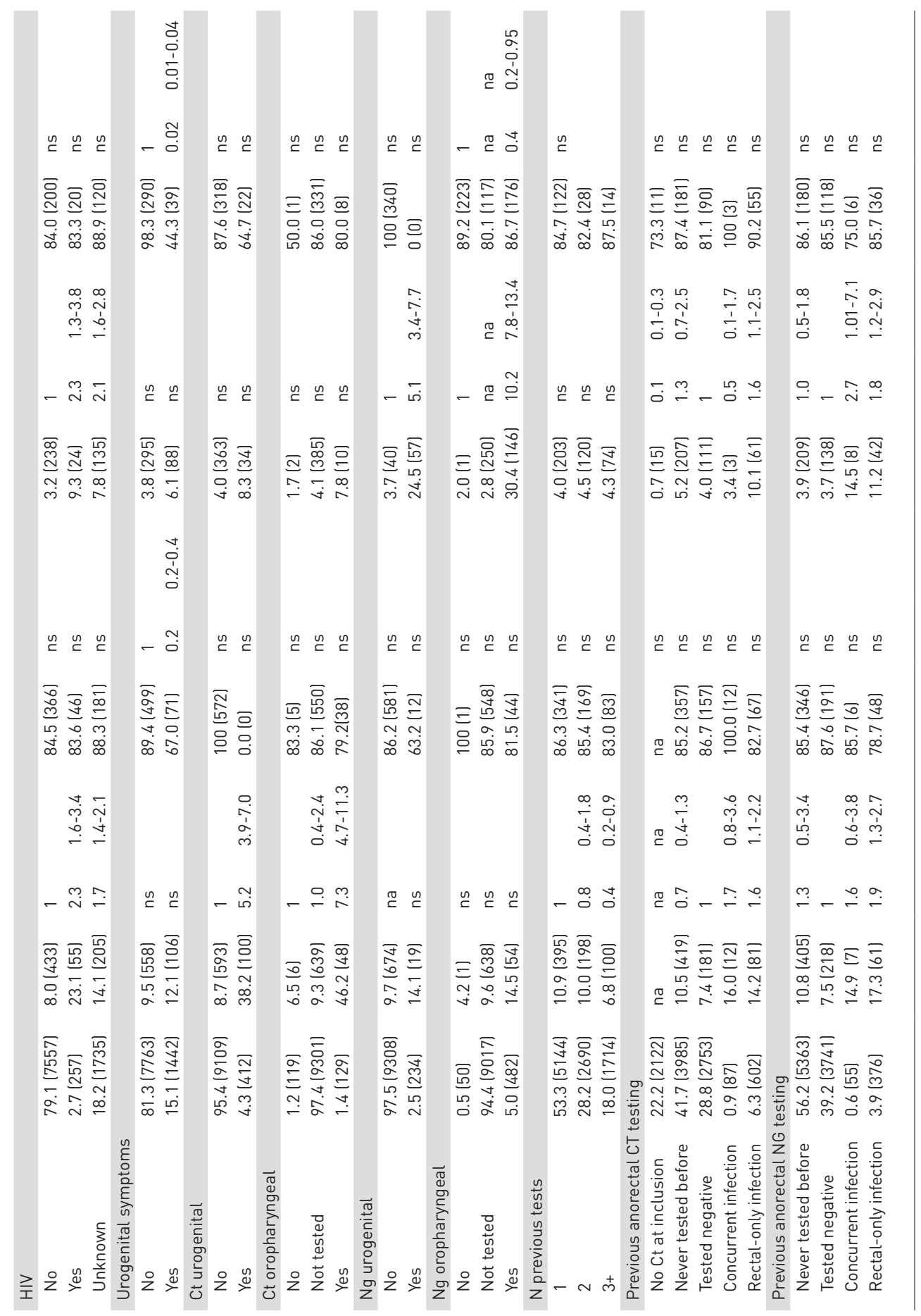




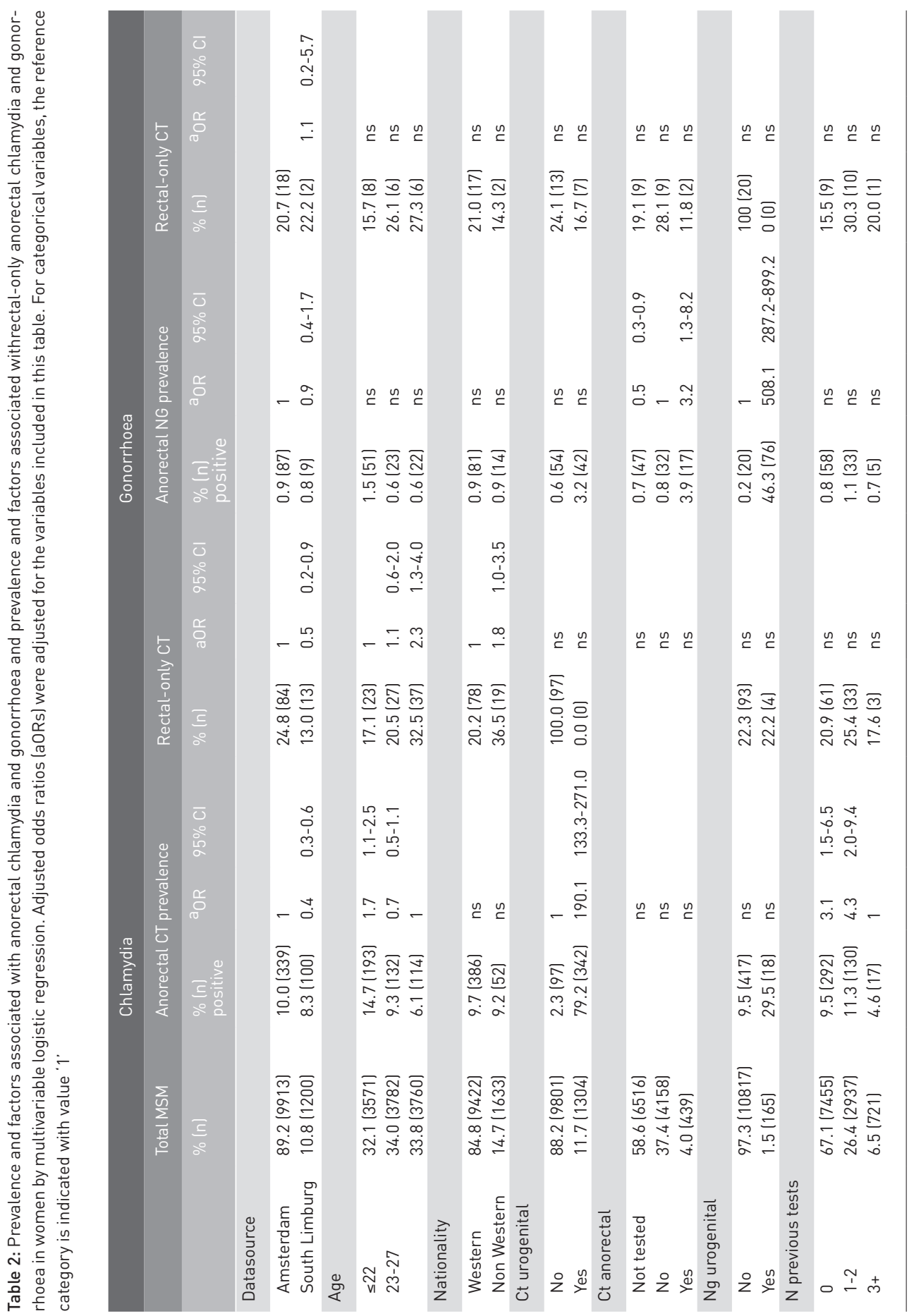




\section{Anorectal CT and NG prevalence}

The prevalence of anorectal CT was 9.8\% (693/7094) in MSM and 9.5\% (439/4597) in women $(p=0.86)$. Anorectal CT prevalence among women who were routinely universally tested was $10.4 \%$ (20/192), for selective testing this was $9.5 \%(419 / 4405)(p=0.68)$. The prevalence of anorectal NG in MSM was 4.2\% (397/9534), in women this was $0.9 \%(96 / 10972)(p<0.001)$.

\section{Prevalence of rectal-only infections}

In MSM, 162 urogenital and 693 anorectal CT infections and 176 urogenital and 397 anorectal NG infections were diagnosed. In women, 90 urogenital and 439 anorectal CT infections were diagnosed, and 88 urogenital and 96 anorectal NG infections were diagnosed (figure 2). Among anorectal CT positive MSM, 85.6\% (593/693) had rectal-only infections, that is, without a simultaneous concurrent urogenital CT infection. For women this proportion was $22.1 \%$ (97/439) $(p<0.001)$. Among anorectal NG positive MSM, 85.6\% (340/397) had rectal-only infections, for women this proportion was $20.8 \%(20 / 96)(p<0.001)$.

Figure 2: Anatomic site distribution of CT or NG positive MSM and women both tested at urogenital and anorectal sites

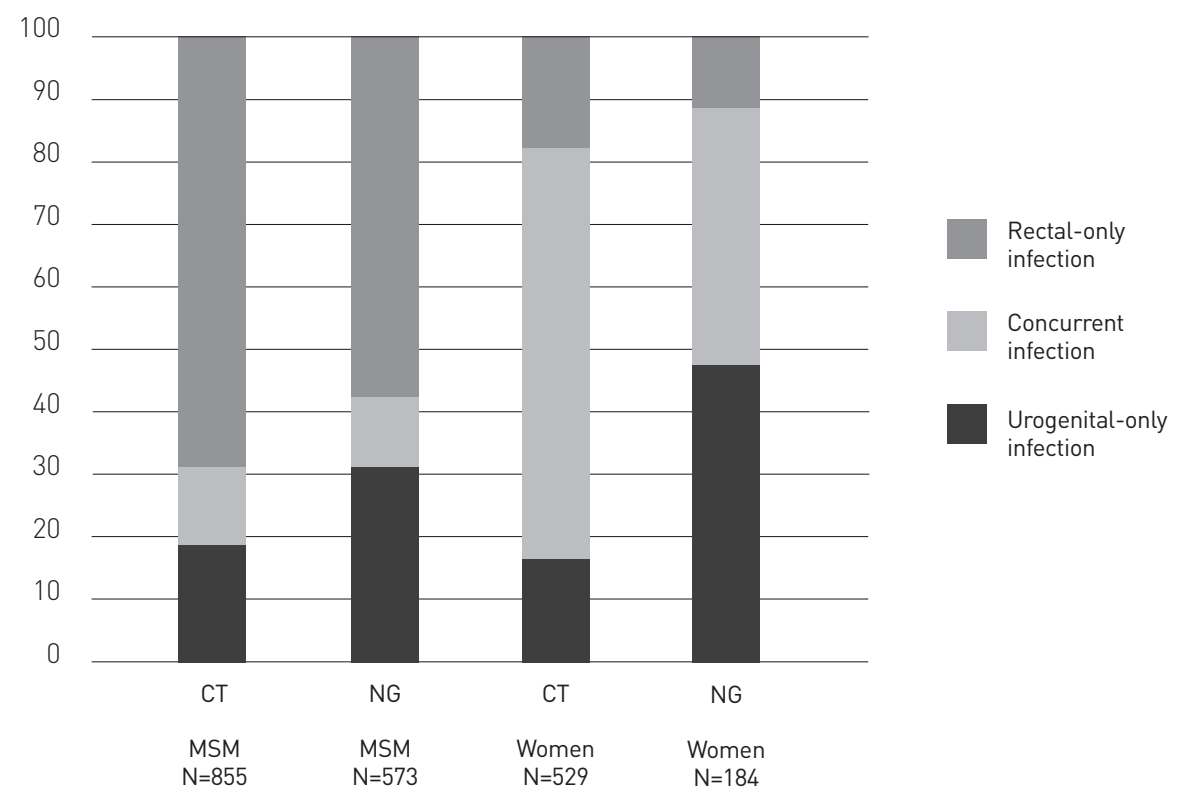




\section{Factors associated with anorectal CT and NG}

\section{MSM}

Factors univariately associated with anorectal CT or NG are presented in table 3, independent factors positively associated with anorectal CT or NG in MSM are presented in table 1. We assessed which factors would yield the highest number of CT or NG infections in MSM if targeted in screening by taking into account the absolute number of infections per factor. The largest share of anorectal CT infections was found in MSM exclusively having sex with men, men of a younger age, those reporting having had 3 or more sex partners, who had been warned for STIs by an (ex) partner, or were not always using a condom when practising anal sex (figure 3 ). Figure 3 presents the relative share of anorectal infections per factor. For example, screening all MSM who were warned for STIs by an (ex) partner would yield about $50 \%$ of all anorectal CT infections in MSM. This factor adds significantly to finding anorectal infections (OR 2.0 on X axis), but does not contribute significantly to finding rectal-only CT (OR 1.0 on Y axis). In total, one or more of the factors presented in figure 3 was applicable to $98.3 \%$ (6973/7094) of MSM. Our data show that using this as a testing algorithm would diagnose $99.3 \%(n=691)$ of anorectal CT infections in MSM.

The largest share of anorectal NG infections was found in MSM who were exclusively having sex with men, who were of a younger age, who had 3 or more sex partners, who had been warned by an (ex) partner, or were experiencing urogenital symptoms (figure 3). In total, one or more of these factors applied to $97.1 \%$ (9261/9534) of MSM. Using this as a testing algorithm would diagnose $99.7 \%(n=396)$ of anorectal NG infections in MSM. .

\section{Women}

Factors univariately associated with anorectal CT or NG are presented in table 4, independent factors positively associated with anorectal CT or NG in women are presented in table 2. We assessed which factors would yield the highest number of CT or NG infections in women if targeted in screening by taking into account the absolute number of infections per factor. The largest share of anorectal CT infections was found in women of a younger age, women who had not visited an STI clinic in the previous 2 years, women who had been warned by an (ex) partner, or those not always using a condom when practising anal sex (figure 3). In total, $83.9 \%$ (3855/4597) of women had one of these factors or a combination thereof. Using this as a testing algorithm would diagnose $93.8 \%(n=412)$ of anorectal NG infections in women. The largest share of anorectal NG infections was found in women of a younger age, those having had anal sex, or women who had been warned by an (ex) partner (figure 3). In total, 62.8\% (6895/10972) of women had one of these factors of a combination thereof. Using this as a testing algorithm would diagnose $85.4 \%(n=82)$ of anorectal NG infections in women. 
Figure 3: Bubble plot depicting the factors univariately associated with anorectal CT and NG and rectal-only CT and NG in MSM and women including their relative share in the total number of anorectal CT and NG infections
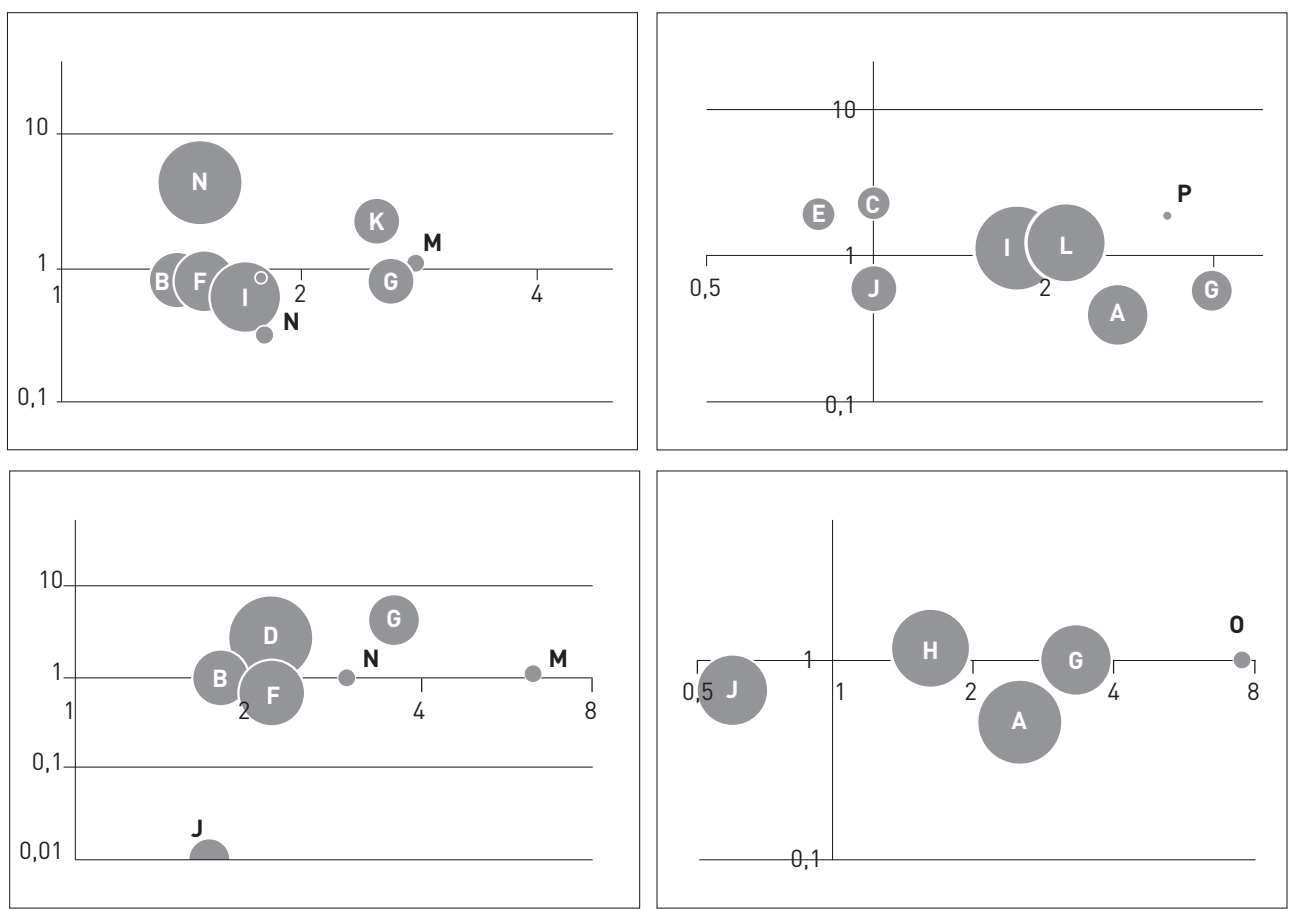

The $X$-axis represents the odds ratio of anorectal CT or NG, the $Y$-axis represents the odds ratio of rectal-only CT or NG. The bubble represents the relative share in percentages of anorectal CT and NG infections per associated factor. The variable anal sex was not used for $C T$ to prevent bias by testing indication.

\begin{tabular}{llll} 
Letter & Variable & Letter & Variable \\
\hline A & Age $\leq 22$ Women & I & Not always used a condom during anal sex \\
\hline B & Age $\leq 32$ MSM & J & Having urogenital symptoms \\
\hline C & Non Western nationality & K & Having anorectal symptoms \\
\hline D & MSM exclusively having sex with men & L & No previous CT/NG test \\
E & CSW & M & 2+ previous NG infections \\
\hline F & 3+ sex partners & N & 2+ previous CT infections \\
G & Being warned by lex)partners & 0 & Having had previous concurrent NG \\
\hline H & Had anal sex (NG) & P & Having had previous rectal-only CT \\
\hline
\end{tabular}




\section{Factors associated with rectal-only CT and NG}

MSM

Factors independently positively associated with rectal-only CT were: exclusively having sex with men, not being a CSW in the past 6 months, and absence of urogenital symptoms. Exclusively having sex with men was the only factor which was also associated with anorectal CT. The largest share of rectal-only CT infections was found in MSM of a younger age, those exclusively having sex with men or MSM who had been warned by an (ex) partner (table 1 and figure 3). Factors independently associated with rectal-only NG were: having been warned for STIs by an (ex) partner, absence of an oropharyngeal NG infection, and absence of urogenital symptoms. On the contrary, an oropharyngeal NG infection was associated with anorectal NG infection. Also having been warned for STIs by an (ex) partner was also associated with anorectal NG, however absence of urogenital symptoms was not associated. The largest share of rectal-only NG infections was found in MSM of a younger age, those exclusively having sex with men or those who had been warned by an (ex) partner (table 1 and figure 3).

\section{Women}

Factors independently associated with rectal-only CT infection were: older age ( $\geq 28$ years), non-Western nationality, and being a CSW. None of these factors were associated with anorectal CT. On the contrary, younger age was associated with anorectal CT. The largest share of rectalonly CT infections were found in older women, women with non Western nationality, women who reported to be a CSW, women who did not always use a condom when practising anal sex, and women who had not visited an STI clinic in the previous 2 years (table 2 and figure 3). No factors were independently associated with rectal-only NG infection in our multivariable analyses.

\section{Discussion}

In the present study with 20662 unique individuals, only a few factors were found to be weakly associated with rectal-only infections, making it difficult to perform selective anorectal screening based on a priori patient characteristics. On the contrary, a wide range of factors was found to be associated with CT and/or NG positivity. Among CT or NG positive MSM, the majority had a rectal-only infection, whereas the majority of women had a concurrent urogenital infection.

We found that the prevalence of anorectal CT in STI outpatient clinic attendees was substantial, at $10 \%$, and comparable for both MSM and women. Anorectal NG prevalence was higher in MSM ( $4 \%)$ as compared to women (1\%). The prevalence of anorectal CT and NG in this study is consistent with the prevalence described earlier[15,19,20,23,27]. We found multiple independent associations with anorectal CT and NG infections in MSM and women, which is also consis- 
tent with the findings of other studies[8,9,11,12,18-20,23,29,30]. Most previous studies have focused on anorectal CT and NG in MSM; only a few compare MSM and women[13-15,26]. Here, we show that anorectal CT and NG infections are found both in women and MSM attending STI clinics. However, in women, concurrent urogenital and anorectal CT (78\%) and NG infections $(79 \%)$ are detected more frequently than rectal-only infections in contrast to MSM with $14 \%$ concurrent CT and NG infections. Possible explanations include autoinoculation via vaginal secretions[18,21,23,27], or concurrent transmission during sex.

This study focussed on rectal-only CT and NG infections. It is important to identify rectal-only infections because these remain unnoticed in urogenital site only screening algorithms and, in the case of $\mathrm{CT}$, are possibly sub optimally treated (with single dose azithromycin) when cooccurring with genital CT infection[31,32]. Although the clinical relevance of anorectal infections in women is still largely unknown, it has been suggested that adequate treatment can help limit the spread of CT and NG in the general population[18,19,23], and can reduce susceptibility for HIV infection.

In this study, older age and non-Western nationality were associated with rectal-only infections in women. A Canadian study found older age, and being warned by an (ex)partner for STI to be associated with rectal-only CT infection in women[33]. On the other hand, a US study found young age ( $<18$ years) to be associated with rectal-only CT[15]. This indicates that results are inconclusive and it would be hard to target rectal-only infections in women in practice based on a priori patient characteristics. No associations were found with rectal-only NG in women, as was also the case in a study by Trebach et al.[15]. This may be a result of low numbers of rectalonly NG infections in women ( $n=20$ in our study and $n=50$ in the study of Trebach et al.)[15].

In MSM, exclusively having sex with men was associated with both anorectal CT and rectal-only CT. This was the only factor positively associated with rectal-only CT infections in MSM, which makes it hard to target rectal-only infections in MSM in practice based on a priori patient characteristics, as was the case with women. However, encouraging MSM, especially MSM exclusively having sex with men, for anorectal testing yields both anorectal CT and rectal-only anorectal CT. Not having urogenital symptoms and being a CSW were protective for rectal-only CT in this study. A study by Gratrix et al. found that being asymptomatic was associated with a rectal-only CT infection in MSM[34]. However, the absence of urogenital symptoms does not rule out the possibility of a rectal-only CT infection.

Notably, reporting anal sex and anal symptoms were not associated with rectal-only infections in both MSM and women.

When examining data from previous consultations in the past two years in MSM, we found that previous rectal-only CT and NG were associated with anorectal CT. Both previous concurrent 
and rectal-only NG and previous rectal-only CT infections were associated with anorectal NG. A study by Bernstein et al. reported an increased risk of HIV infection among MSM with rectal infections in the past two years[35]. However, retesting anorectal NG positive MSM would yield only a minority of all anorectal NG infections. Moreover, MSM and women who had a previous rectal-only CT or NG infection were not more likely to have a rectal-only infection.

Selective testing on indication for anorectal CT and NG is currently recommended in the guidelines $[5,6]$ and already widely practised. Previous studies show that anal sex is not associated with anorectal CT in MSM and women, but is associated with anorectal NG[14,20]. Our results indicate that screening all MSM and women who report anal sex or symptoms (selective testing) remains important in order to detect anorectal CT and NG including rectal-only infections. The majority of the study population was tested routinely universally for anorectal NG. In addition to selective testing for anorectal NG, including factors associated with anorectal NG in the testing algorithm could help to detect additional anorectal NG infections in both MSM and women.

Our study has several limitations. A large number of unique individuals were included in this study ( $N=20662$ ). However, prevalence of anorectal NG infections in women is low $(0.9 \%)$ which might have been too low to reach statistical significance when examining associated factors. If individuals had been tested at another care provider in the two years prior to consultation, for example by their general practitioner (GP), this was not taken into account. This could have led to an overestimation of individuals who had not been tested in the two years prior to consultation. However, we presume this bias to be minimal as anorectal testing is limited at GP surgeries[36]. A further limitation of our study is that anorectal test algorithms differed between the two STI clinics. In Amsterdam, all attendees were tested systematically for anorectal NG, but anorectal CT testing was based on reports of anal sex and/or symptoms. In South Limburg, all MSM were routinely tested for anorectal CT and NG and women partially systematically (between May and December 2012), and partially based on report of anal sex and/or symptoms. Such selective testing on indication misses about half of anorectal infections in both MSM[14] and women[20]. It is therefore likely that some anorectal infections were missed using these testing criteria. Possibly, these missed anorectal infections were rectal-only infections, which would bias our results. Moreover, we could have missed factors associated with rectal-only infections since routine universal anorectal screening was missing. Additionally, culture was used for anorectal NG tests in Amsterdam, which might have missed NG infections due to lower sensitivity of culture compared to NAAT. However, the anatomic site distribution of single-site and concurrent CT and NG in MSM and women is comparable with other studies who used routine universal anorectal screening. Moreover, factors associated with anorectal CT and NG found here were comparable with those reported in other studies $[8,9,11,12,15,18-20,23,29,30]$. 


\section{Conclusions}

In conclusion, prevalence of anorectal CT is substantial in both MSM and women, and prevalence of NG is substantial in MSM. The majority of MSM have rectal-only CT and NG infections, in contrast to women, who are more likely to have concurrent urogenital and anorectal infections. Only a few factors were associated with rectal-only infections, and this makes anorectal screening algorithms based on a priori patient characteristics for MSM and women challenging, because of low discriminatory power. We recommend further research is carried out to inform and optimise anorectal CT and NG case finding.

\section{Acknowledgments}

We would like to thank the staff of the Amsterdam and South Limburg STI clinics for their valuable contribution to data collection. 


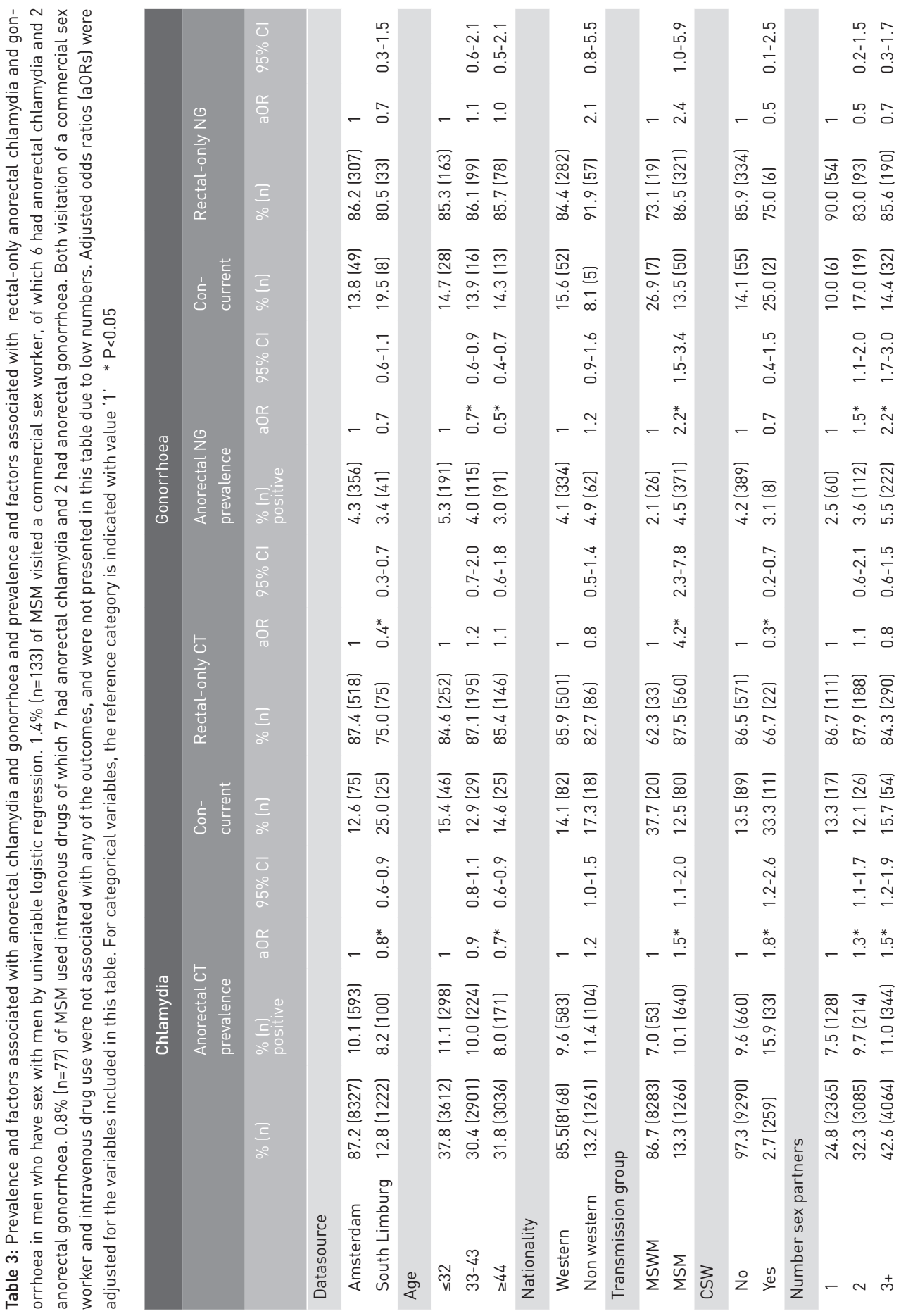




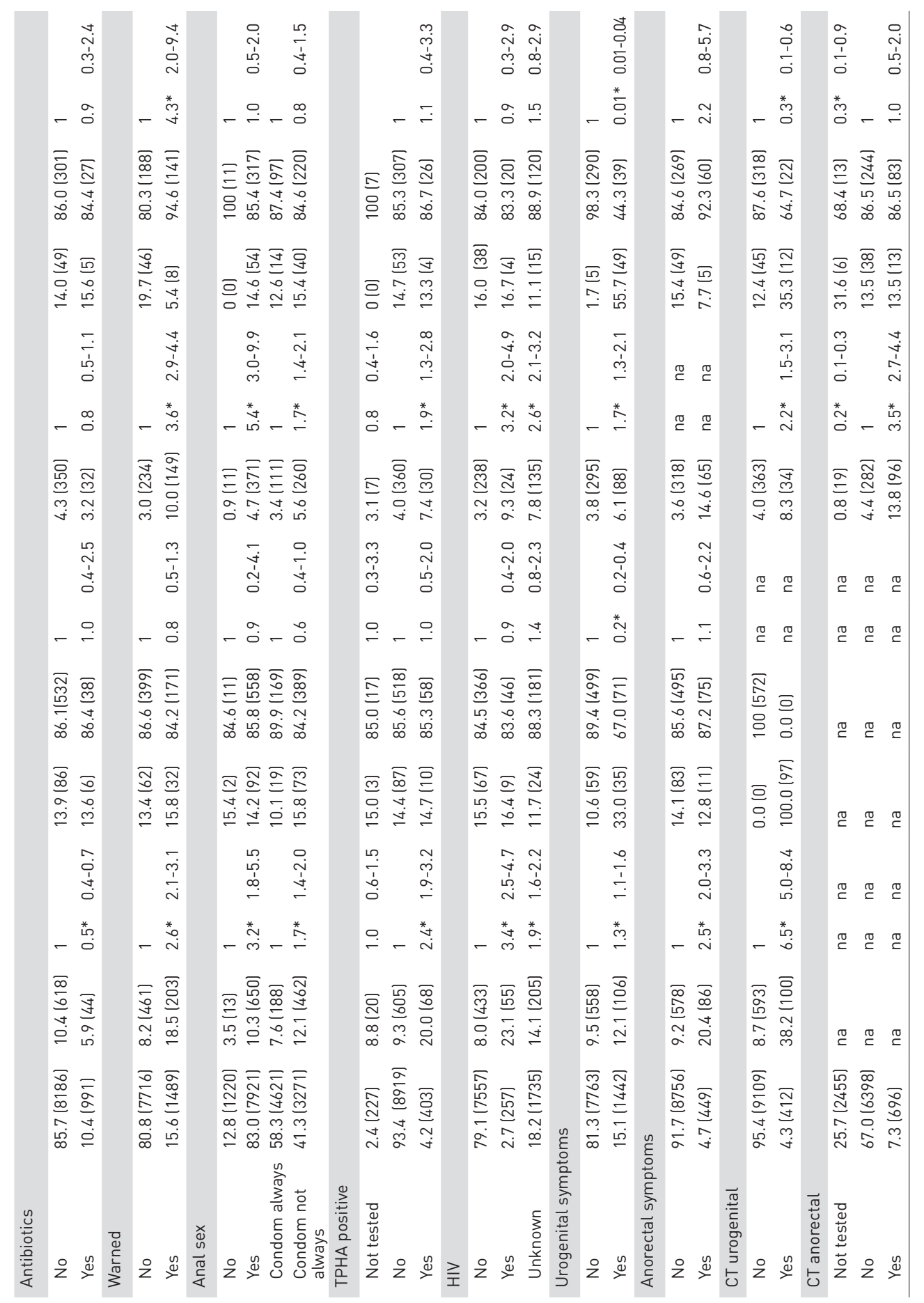




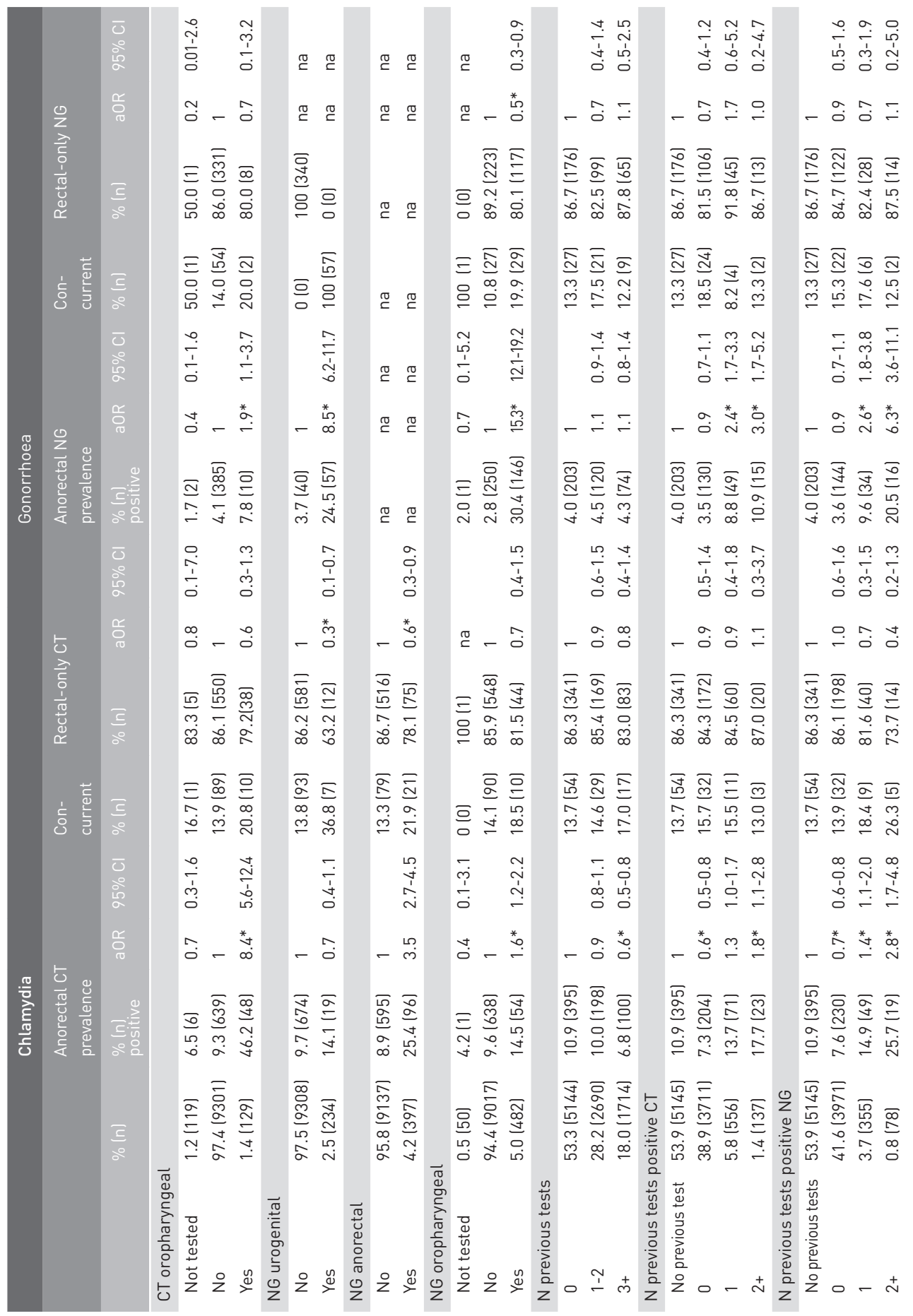




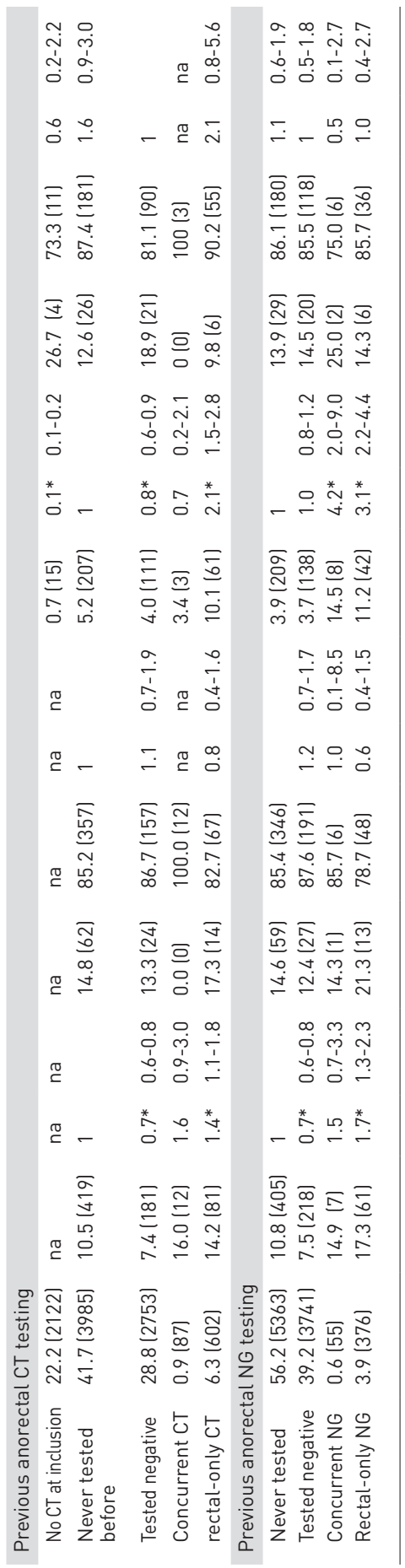




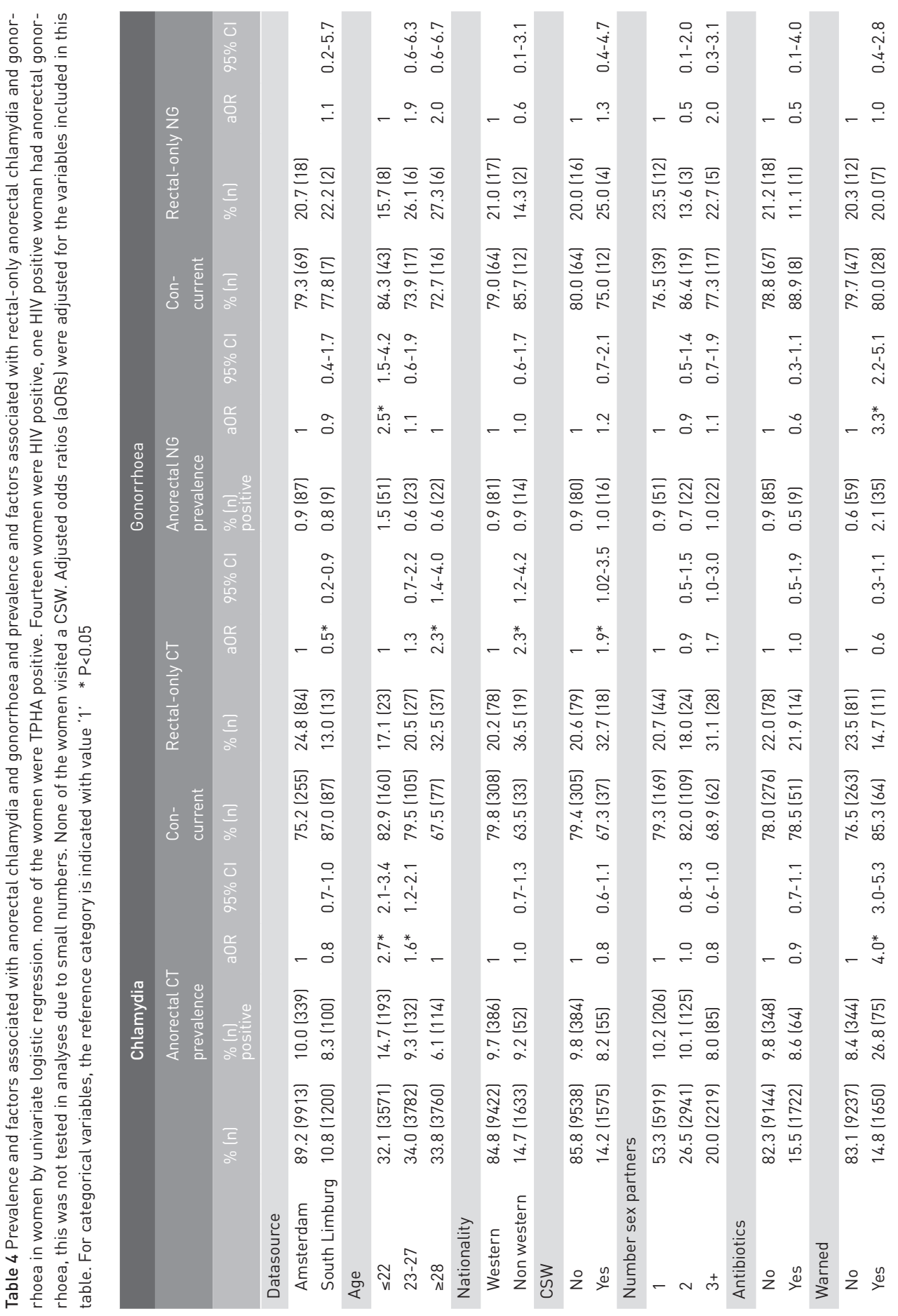




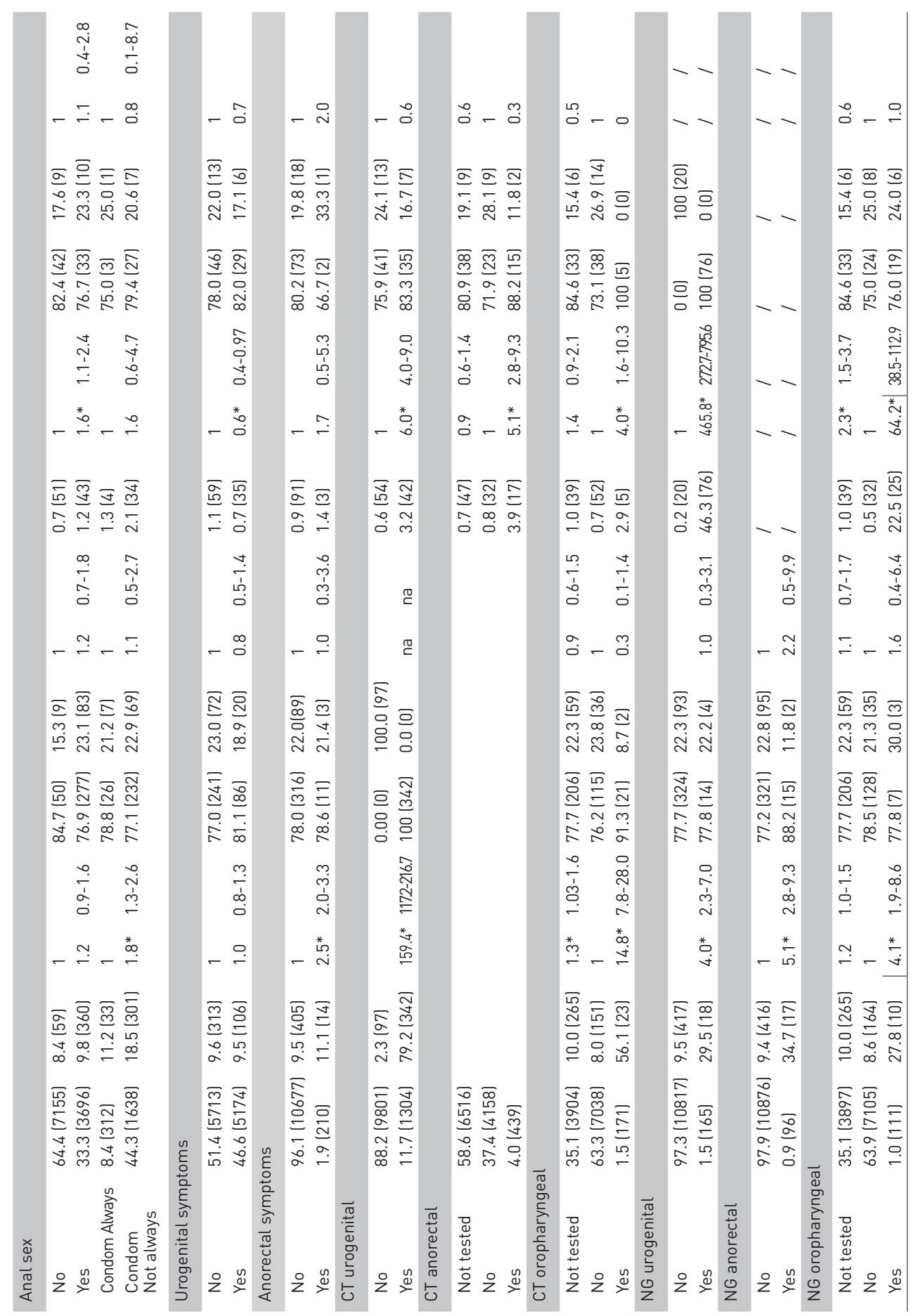




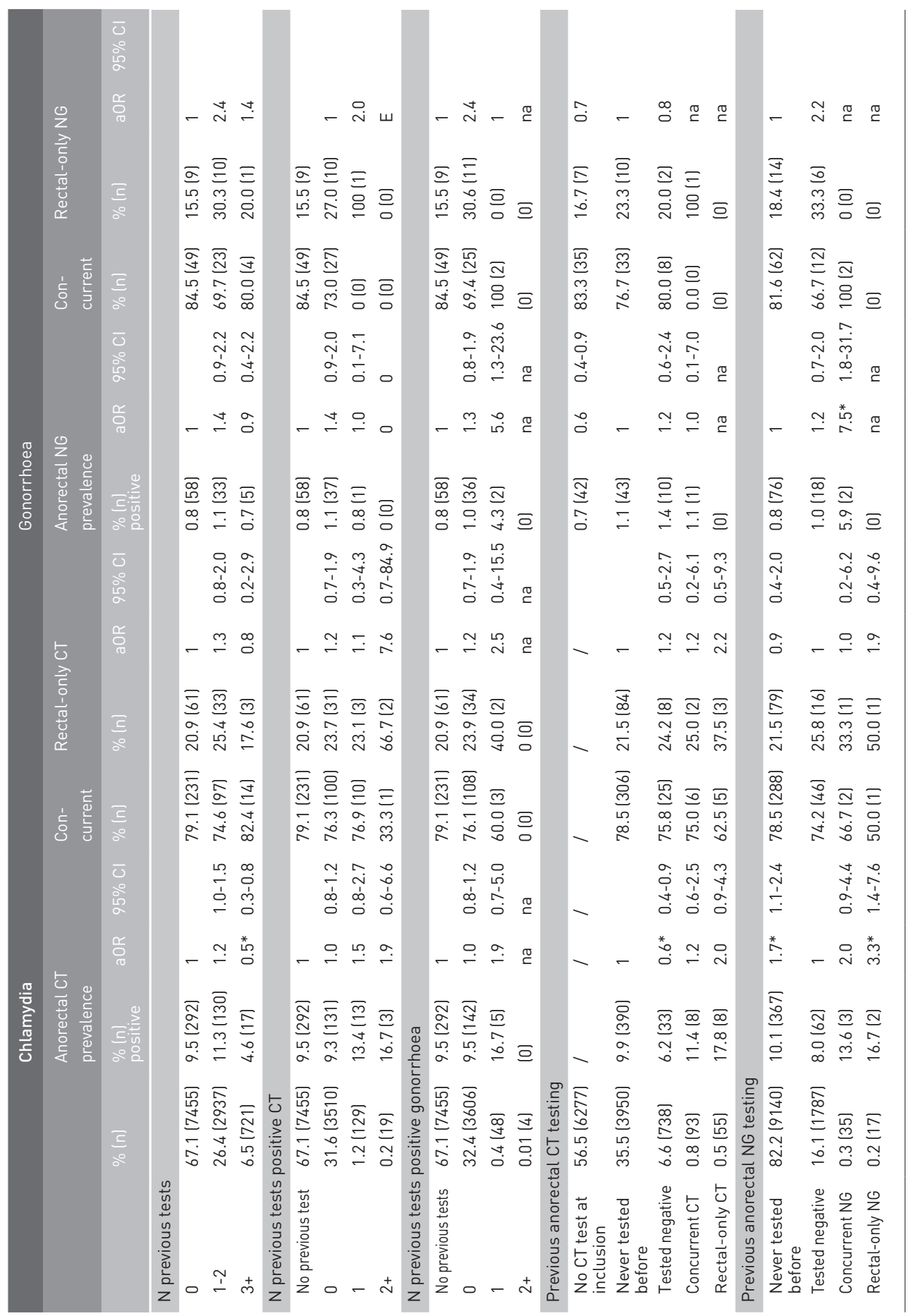




\section{References}

1. Schachter J, Philip SS. Testing men who have sex with men for urethral infection with Chlamydia trachomatis and Neisseria gonorrhoeae is only half the job, and we need the right tools. Sex Transm Dis. 2011;38: 925-927.

2. Papp JR, Schachter J, Gaydos CA, Van Der Pol B. Recommendations for the LaboratoryBased Detection of Chlamydia trachomatis and Neisseria gonorrhoeae - 2014.

3. Koedijk FD, van Bergen JE, DukersMuijrers NH, van Leeuwen AP, Hoebe CJ, van der Sande MA. The value of testing multiple anatomic sites for gonorrhoea and chlamydia in sexually transmitted infection centres in the Netherlands, 2006-2010. Int J STD AIDS. 2012;23: 626-631.

4. Fleming DT, Wasserheit JN. From epidemiological synergy to public health policy and practice: the contribution of other sexually transmitted diseases to sexual transmission of HIV infection. Sex Transm Infect. 1999;75: 3-17.

5. Workowski KA, Berman S. Sexually transmitted diseases treatment guidelines, 2010. MMWR Recomm Rep.59: 1-110.

6. de Vries HJC, van Doornum GJJ, Bax CJ Multidisciplinaire Richtlijn Seksueel Overdraagbare Aandoeningen voor de 2e Lijn, Nederlandse Vereniging voor Dermatologie en Venereologie. http://www.tinyurl. soarichtlijn2012

7. Nwokolo NC, Dragovic B, Patel S, Tong CYW, Barker G, Radcliffe K. (2014) 2014 Draft UK national guideline for the management of genital infection with Chlamydia trachomatis.

8. Dudareva-Vizule S, Haar K, Sailer A,
Wisplinghoff $\mathrm{H}$, Wisplinghoff F, Marcus U. Prevalence of pharyngeal and rectal Chlamydia trachomatis and Neisseria gonorrhoeae infections among men who have sex with men in Germany. Sex Transm Infect. 2013;90: 46-51.

9. Kent CK, Chaw JK, Wong W, Liska S, Gibson S, Hubbard G, et al. Prevalence of rectal, urethral, and pharyngeal chlamydia and gonorrhea detected in 2 clinical settings among men who have sex with men: San Francisco, California, 2003. Clin Infect Dis. 2005;41: 67-74.

10. Patton ME, Kidd S, Llata E, Stenger M, Braxton J, Asbel L, et al. Extragenital gonorrhea and chlamydia testing and infection among men who have sex with men--STD Surveillance Network, United States, 2010-2012. Clin Infect Dis. 2014;58: 1564-1570.

11. Peters RP, Verweij SP, Nijsten N, Ouburg S, Mutsaers J, Jansen CL, et al. Evaluation of sexual history-based screening of anatomic sites for chlamydia trachomatis and neisseria gonorrhoeae infection in men having sex with men in routine practice. BMC Infect Dis.

2011;11: 203.

12. Annan NT, Sullivan AK, Nori A, Naydenova $P$, Alexander S, McKenna A, et al. Rectal chlamydia--a reservoir of undiagnosed infection in men who have sex with men. Sex Transm Infect. 2009;85: 176-179.

13. van Liere GA, Hoebe CJ, Dukers-Muijrers NH. Evaluation of the anatomical site distribution of chlamydia and gonorrhoea in men who have sex with men and in high-risk women by routine testing: cross-sectional study 
revealing missed opportunities for treatment strategies. Sex Transm Infect. 2013;90: 58-60.

14. van Liere GA, Hoebe CJ, Niekamp AM, Koedijk FD, Dukers-Muijrers NH. Standard symptomand sexual history-based testing misses anorectal Chlamydia trachomatis and neisseria gonorrhoeae infections in swingers and men who have sex with men. Sex Transm Dis. 2013;40: 285-289.

15. Trebach JD, Chaulk CP, Page KR, Tuddenham S, Ghanem KG. Neisseria gonorrhoeae and Chlamydia trachomatis Among Women Reporting Extragenital Exposures. Sex Transm Dis. 2015;42: 233-239.

16. Li JH, Cai YM, Yin YP, Hong FC, Shi MQ, Feng TJ, et al. Prevalence of anorectal Chlamydia trachomatis infection and its genotype distribution among men who have sex with men in Shenzhen, China. Jpn J Infect Dis. 2011;64: 143-146.

17. Turner AN, Reese PC, Ervin M, Davis JA, Fields KS, Bazan JA. HIV, rectal chlamydia, and rectal gonorrhea in men who have sex with men attending a sexually transmitted disease clinic in a midwestern US city. Sex Transm Dis.40: 433-438.

18. Barry PM, Kent CK, Philip SS, Klausner JD. Results of a program to test women for rectal chlamydia and gonorrhea. Obstet Gynecol. 2010;115: 753-759.

19. Javanbakht $M$, Gorbach $P$, Stirland A, Chien $M$, Kerndt $P$, Guerry S. Prevalence and correlates of rectal Chlamydia and gonorrhea among female clients at sexually transmitted disease clinics. Sex Transm Dis. 2012;39: 917-922.
20. van Liere GA, Hoebe CJ, Wolffs PF, DukersMuijrers NH. High co-occurrence of anorectal chlamydia with urogenital chlamydia in women visiting an STI clinic revealed by routine universal testing in an observational study; a recommendation towards a better anorectal chlamydia control in women. BMC Infect Dis. 2014;14: 274.

21. Sethupathi M, Blackwell A, Davies H. Rectal Chlamydia trachomatis infection in women. Is it overlooked? Int J STD AIDS. 2009;21: 93-95.

22. Bazan JA, Carr Reese P, Esber A, Lahey S, Ervin $M$, Davis JA, et al. High prevalence of rectal gonorrhea and Chlamydia infection in women attending a sexually transmitted disease clinic. J Womens Health (Larchmt).24: 182-189.

23. Hunte T, Alcaide M, Castro J. Rectal infections with chlamydia and gonorrhoea in women attending a multiethnic sexually transmitted diseases urban clinic. Int J STD AIDS. 2010;21: 819-822.

24. Ladd J, Hsieh YH, Barnes M, Quinn N, Jett-Goheen M, Gaydos CA. Female users of internet-based screening for rectal STIs: descriptive statistics and correlates of positivity. Sex Transm Infect. 2014;90: 485-490.

25. Shaw SG, Hassan-Ibrahim M, Soni S. Are we missing pharyngeal and rectal infections in women by not testing those who report oral and anal sex? Sex Transm Infect. 2013;89: 397.

26. Bax CJ, Quint KD, Peters RP, Ouburg S, Oostvogel PM, Mutsaers JA, et al. Analyses of multiple-site and concurrent Chlamydia trachomatis serovar infections, and serovar tissue tropism for urogenital versus rectal 
specimens in male and female patients. Sex

Transm Infect. 2011;87: 503-507.

27. Peters RP, Nijsten N, Mutsaers J, Jansen CL, Morre SA, van Leeuwen AP. Screening of oropharynx and anorectum increases prevalence of Chlamydia trachomatis and Neisseria gonorrhoeae infection in female STD clinic visitors. Sex Transm Dis. 2011;38: 783-787.

28. Versteeg B, van Rooijen MS, Schim van der Loeff MF, de Vries HJ, Bruisten SM. No indication for tissue tropism in urogenital and anorectal Chlamydia trachomatis infections using high-resolution multilocus sequence typing. BMC Infect Dis. 2014;14: 464.

29. Manavi K, McMillan A, Young $H$. The prevalence of rectal chlamydial infection amongst men who have sex with men attending the genitourinary medicine clinic in Edinburgh. Int J STD AIDS. 2004;15: 162-164.

30. Geisler WM, Morrison SG, Bachmann LH. Absence of lymphogranuloma venereum strains among rectal Chlamydia trachomatis outer membrane protein. A genotypes infecting women and men who have sex with men in Birmingham, Alabama. Sex Transm Dis. 2008;35: 856-858.

31. Hathorn E, Opie C, Goold P. What is the appropriate treatment for the management of rectal Chlamydia trachomatis in men and women? Sex Transm Infect. 2012;88: 352-354.

32. Khosropour CM, Dombrowski JC, Barbee LA, Manhart LE, Golden MR. Comparing azithromycin and doxycycline for the treatment of rectal chlamydial infection: a retrospective cohort study. Sex Transm Dis. 2014;41: 79-85.
33. Gratrix J BJ, Egan C, Singh AE, Drews S, Read RR. Prevalence and Correlates of rectal-only chlamydia infection at two Canadian STI clinics. Sex Trans Infect. 2013;89: A152-A153

34. Gratrix J, Singh AE, Bergman J, Egan C, Plitt SS, McGinnis J, et al. Evidence for Increased Chlamydia Case Finding After the Introduction of Rectal Screening Among Women Attending 2 Canadian Sexually Transmitted Infection Clinics. Clin Infect Dis. 2015;60: 398-404.

35. Bernstein KT, Marcus JL, Nieri G, Philip SS, Klausner JD. Rectal gonorrhea and chlamydia reinfection is associated with increased risk of HIV seroconversion. J Acquir Immune Defic Syndr. 2010;53: 537-543.

36. de Coul EL, Warning TD, Koedijk FD. Sexual behaviour and sexually transmitted infections in sexually transmitted infection clinic attendees in the Netherlands, 2007-2011. Int J STD AIDS.25: 40-51. 



$$
\begin{gathered}
\text { Section } \\
\text { bacterial load }
\end{gathered}
$$





\section{Chapter 8}

\section{Anorectal Chlamydia trachomatis load is similar in men who have sex with men and women reporting anal sex}

Geneviève AFS van Liere, Jeanne AMC Dirks, Christian JPA Hoebe,

Petra F Wolffs, Nicole HTM Dukers-Muijrers

PLoS ONE 2015 Aug 11; 10(8). doi:10.1371/ journal.pone.0134991 


\section{Abstract}

\section{Introduction}

Anorectal Chlamydia trachomatis (chlamydia) is frequently diagnosed in men who have sex with men (MSM) and in women but it is unknown whether these infections are comparable in clinical impact and transmission potential. Quantifying bacterial load and identifying determinants associated with high bacterial load could provide more insight.

\section{Methods}

We selected a convenience sample of MSM who reported anal sex $(n=90)$ and women with concurrent urogenital/anorectal chlamydia who reported anal sex $(n=51)$ or did not report anal sex ( $n=61)$ from the South Limburg Public Health Service's STI unit. Bacterial load (Chlamydia/ml) was quantified for all samples and log transformed for analyses. Samples with an unquantifiable human leukocyte antigen $(n=9)$ were excluded from analyses, as they were deemed inadequately sampled.

\section{Results}

The mean log anorectal chlamydia load (3.50) was similar for MSM and women who reported having anal sex $(3.80, P=0.21)$. The anorectal chlamydia load was significantly higher in these groups than in women who did not report having anal sex (2.76, $P=0.001)$. Detectable load values ranged from 1.81-6.32 chlamydia/ml for MSM, 1.74-7.33 chlamydia/ml for women who reported having anal sex and 1.84-6.31 chlamydia/ml for women who did not report having anal sex. Symptoms and several other determinants were not associated with anorectal chlamydia load.

\section{Discussion}

Women who did not report anal sex had lower anorectal loads, but they were within a similar range to the other two groups. Anorectal chlamydia load was comparable between MSM and women who reported anal sex, suggesting similar transmission potential. 


\section{Introduction}

Anorectal Chlamydia trachomatis (chlamydia) is frequently diagnosed in sexually transmitted infection (STI) clinics. International guidelines advise using nucleic acid amplification tests (NAAT) to test anorectal specimens because they are more sensitive than the use of culture $[1,2]$. Moreover, self-collected rectal samples are as feasible, valid, and acceptable for testing as provider-collected swabs [3,4]. Anorectal testing is mainly indicated in risk groups such as men who have sex with men (MSM); in some countries, guidelines also include women at high risk such as prostitutes, swingers and those with multiple sex partners. Testing is generally guided by report of receptive anal sex and/or anal symptoms $[5,6]$.

Anorectal infections detected by a NAAT in MSM are generally assumed to be real infections and are given adequate treatment to halt transmission and reduce complications in individuals. Additionally, in recent years, several published studies have recommended anorectal testing for women because of the substantial prevalence of anorectal chlamydia among them (6-15\%) [7-9]. The prevalence of anorectal chlamydia in women is strikingly similar to that in MSM (7-14\%) [10-12]. Women do frequently report anal sex (11-26\%) as do MSM [9,13-15]. Nonetheless, recent studies using routine anorectal testing have found that over half to two-thirds of anorectal chlamydia infections were observed in women and MSM who did not report anal sex $[9,16,17]$. This finding fuels international debate about who to test for anorectal chlamydia infections.

Although the prevalence is similar between MSM and women, anorectal chlamydia infections in women are mainly concurrent with urogenital infections, in contrast to MSM $[8-10,12,18,19]$. This may give rise to questioning whether anorectal infections in MSM and women detected by NAAT are comparable in clinical impact and transmission potential. The clinical impact may be considered in terms of symptoms. The majority of anorectal chlamydia infections in MSM and women are asymptomatic. Transmission potential could be carefully considered in terms of bacterial load (for example high loads indicate a higher transmission potential for viral STIs) [20]. Therefore, targeting individuals with high chlamydia loads could have an added value in practice.

To date, only a few studies have used a NAAT to report on anorectal load [15,21,22], and none of these studies have compared anorectal loads between MSM and women. Yet, quantifying bacterial load and knowing which determinants are associated with high bacterial load could provide a unique insight into possible clinical (i.e. symptoms) and public health (i.e. transmission) aspects of anorectal infections. Additionally, comparing bacterial load and associated determinants between MSM and women could help to ascertain whether their anorectal infections are equally severe in terms of symptoms and transmission. Moreover, anorectal load could differ between women who report anal sex and women who do not report anal sex, due to different transmission routes (i.e. autoinoculation from vaginal secretions in women who do not report 
anal sex). This study quantified bacterial load in chlamydia positive anorectal samples from MSM who reported anal sex (tested as recommended in STI guidelines [5]) and from women with concurrent urogenital chlamydia, and assessed determinants associated with bacterial load.

\section{Methods}

\section{Study population and procedures}

The South Limburg Public Health Service's STI unit provides more than 6000 consults annually, offering free examination and treatment at three regional outpatient STI clinics. Clients are routinely tested at urogenital sites for Chlamydia trachomatis and Neisseria gonorrhoeae. Men who report having had sex with men in the past six months are also routinely tested anorectally. Until May 2012, women were anorectally tested based on self-report of anorectal symptoms and/or anal sex. From May 2012, all women were routinely tested anorectally.

Specimens were self-collected vaginal swabs, anorectal swabs and urine. Trained STI nurses asked patients to take a self-collected anorectal swab and provided them with verbal instructions and a diagram (i.e. insert the swab $2.5 \mathrm{~cm}$ into the rectum, rotate for 5-10 seconds, and place the swab in the capped tube). Specimens were processed at the department of Medical Microbiology at Maastricht University Medical Center + (Maastricht, The Netherlands) using nucleic acid amplification tests (NAAT) [polymerase chain reaction PCR; Cobas Amplicor until 2012 and afterward Cobas 4800, both from Roche Diagnostics, San Francisco, CA]. Serum was tested for Treponema pallidum hemagglutination antigen (TPHA) and HIV (anti-HIV[1/2], Axsym; Abbott Laboratories, Chicago, IL). Reactive samples were confirmed using Western blot (HIVblot 2.2; Genelabs Diagnostics, Science Park, Singapore), according to the manufacturer's protocol. In addition to testing, trained study nurses took a standardised medical and sexual history at each consult, which included demographic data, self-reported symptoms, sexual behaviour in the preceding six months and antibiotic use in the past month. Anal symptoms (proctitis) included rectal discharge, bleeding, pain, redness, burning sensation or itching. All data were registered in an electronic patient registry.

\section{Study selection}

Between November 2010 and September 2013, 796 anorectal chlamydia infections were diagnosed in MSM who reported anal sex and in women with concurrent urogenital chlamydia. We selected a convenience sample of MSM who reported anal sex and women with concurrent urogenital chlamydia who were 16 years or older and positive for non-LGV anorectal chlamydia for this study; this yielded 211 consults by 194 individuals for further analyses. The study sample (26.5\% from total sample) was selected based on availability and easy access. To assess whether selection bias had occurred in the sample, we used a Chi-square test to compare age, nationality 
and sex between persons who were included in the study and MSM reporting anal sex and women who tested positive for anorectal chlamydia who were not included in the study. Individuals in the study sample were on average younger than those not included (mean 30 years (IQR $22-44)$ versus 34 years (IQR 21-41), $P<0.001$ ). The proportion of individuals with a non-Western nationality was comparable between individuals included and not included $14.8 \% \mathrm{n}=10$ versus $3.8 \% \mathrm{n}=19, \mathrm{P}=0.56)$. The proportion MSM included was smaller compared to women $(19.6 \%$ $n=96$, versus $37.5 \% n=116, P<0.00011$.

This study was approved by the Medical Ethical Committee of the University of Maastricht (METC 11-4-108), who waived the need for consent to be collected from participants. Since retrospective data originated from standard care (in which one can opt-out for the use of their data for scientific research, as approved by METC 11-4-108) and were analyzed anonymously, no further informed consent for data analysis was obtained.

\section{Nucleic acid extraction and quantitative $\mathrm{CT}$ polymerase chain reaction (PCR)}

Total nucleic acids from each $200 \mu \mathrm{l}$ sample were isolated using the QIAamp DNA Mini kit (Qiagen, Hilden, Germany) according to the manufacturer's instructions and eluted in $120 \mu$ l. The eluate was stored at $-20^{\circ} \mathrm{C}$ and thawed once for quantification. Prior to DNA-isolation, an internal extraction and amplification control and a negative extraction control were added to each sample, as described elsewhere $[23,24]$.

Quantitative PCR for CT used primers targeting the single-copy OmpA gene, coding for the major outer membrane protein, as described by Jalal et al. [25]. For eukaryotic cell determination, primers targeting the MHC class II antigen (HLA-DQA1) gene were used, as described by van der Helm et al. [26], as a test for adequacy of the sampling. For absolute quantification, the ompA and HLA-DQA1 PCR products were cloned separately into the pGEM-T easy vector (Promega Corporation, Madison, WI, USA) according to the manufacturer's protocol. Plasmids were isolated using alkaline lysis and purified using phenol/chloroform extraction, as described previously [27]. Logarithmic dilutions covering a 5-log range were made (converted to correspond with 106 to 102 copies/ml in clinical samples).

QPCR was performed with a 7900HT Real-Time PCR System (Applied Biosystems, Foster City, California). In each run, the 96-micro-wells plate contained both dilution series, a negative control and the samples for quantification.

PCR amplification was performed in a total volume of $25 \mu \mathrm{l}$, consisting of a $10 \mu \mathrm{l}$ template and a $15 \mu \mathrm{l}$ reaction mixture containing $12.5 \mu \mathrm{l}$ Absolute qPCR Rox Mastermix (Thermo Scientific, Waltham, USA) and a $2.5 \mu \mathrm{l}$ primer/probe mix consisting of $840 \mathrm{nM}$ forward and reverse primer and $100 \mathrm{nM}$ probe. The amplification reaction consisted of 15 minutes of initial activation at $950 \mathrm{C}$, followed by 42 cycles at $950 \mathrm{C}$ for 15 seconds and $60 \mathrm{oC}$ for 60 seconds. 


\section{Load determination}

Absolute chlamydia loads (log chlamydia/ml) were obtained by entering cycle threshold (Ct) values into a master curve (compiled from over 10 dilution series), and then exponentially transformed. Samples were deemed inadequately sampled and excluded from further analysis when no human cells (HLA-DQA1) were detected or no target could be detected at all; this resulted in excluding 9 of the 211 samples. The initial diagnostic chlamydia screening was done with assays targeting the chlamydial plasmid, which are both present in relative abundance to the singlecopy OmpA- gene used in our load assessment.

For this reason, some samples contained loads below the quantification range of the ompA PCR. These 'low load' samples, which were proven positive at the initial screening, were set to a Ct value $>42$ which resulted in a load of 0.75 log chlamydia/ml[28].

\section{Statistical analyses}

Load values were log transformed for analyses. The study sample consisted of three non-overlapping indication categories based on reported behaviour: (1) MSM who reported anal sex (2) women who reported anal sex and (3) women who did not report anal sex. Demographic data were compared over categories using the student's T-test and ANOVA. Anorectal load values were compared between indication categories (MSM who reported anal sex, women who reported anal sex and women who did not report anal sex) using the student's T-test and univariable linear regression analysis using the second category (women who reported anal sex) as the reference category. Univariable and multivariable linear regression analyses were performed to identify determinants associated with anorectal load overall and separately for each indication category. Additional analyses were performed to assess determinants for high load (i.e., the upper quartile versus the other quartiles). Determinants tested were: sex, age, nationality, number of sex partners in the past six months, sexual preference (for MSM), antibiotic use in the past month, symptoms in the past month (anorectal and urogenital), anal sex in the past six months, and concurrent STI (urogenital chlamydia, anorectal gonorrhoea, syphilis (TPHA positivity) and HIV). Analyses were performed using the SPSS package version 20 (IBM Inc. Somers, New York, USA).

\section{Results}

\section{Characteristics of the study population}

Anal sex was reported by $45.5 \%$ (51/112) of the women. The median age of the MSM ( $n=90)$ was 36 years (IQR 25-47). It was 25 years (IQR 19-27) for women who reported anal sex ( $n=51)$ and 25 years (IQR 20-26) for women who did not report anal sex $(n=61 ; p<0.001)$. The median number of sex partners was 12 (IQR 3-11) for MSM, 4 (IQR 1-4) for women who reported anal sex and 10 (IQR 1-3) for women who did not report anal sex. Anal symptoms were reported by $20.0 \%(n=17)$ 
of MSM, $15.7 \%$ ( $n=8)$ of women who reported anal sex and $3.5 \%(n=2)$ of women who did not report anal sex ( $<0.001)$. In total, $75.8 \%$ ( $n=72$ ) of MSM in the sample reported only having sex with men and $24.2 \%(n=23)$ reported having sex with both men and women. These and other characteristics are described in table 1.

\section{Anorectal chlamydial load in women and MSM}

MSM who reported anal sex had a similar mean log anorectal chlamydia load (3.50) as women who reported anal sex (3.80; figure 1, table 1). In multivariable analyses adjusting for age, anal symptoms, urogenital symptoms and number of sex partners, results remained similar (B 0.29 , $\mathrm{Cl}-0.34-0.92, \mathrm{P}=0.37)$. Anorectal chlamydia load was significantly higher in women who reported anal sex (3.80) than in women who did not report anal sex (2.8, $P=0.001)$. In multivariable analyses, results remained similar ( $\mathrm{B} 1.01, \mathrm{Cl} 0.35-1.67, \mathrm{P}=0.003$ ). When samples with unquantifiable load were excluded from analyses, results comparing load values between groups remained similar. The proportion of samples with an unquantifiable load was $8.9 \%(n=8)$ for MSM who reported anal sex, $9.8 \%(n=5)$ for women who reported anal sex and $27.9 \%(n=17)$ for women who did not report anal sex ( $P=0.03)$. Detectable load values ranged from 1.81-6.32 chlamydia/ml for MSM, 1.74-7.33 chlamydia/ml for women who reported anal sex and 1.84-6.31 chlamydia/ml for women who did not report anal sex (figure 1).

Figure 1: Log-transformed number of cycle threshold per millilitre (Ct/ml) (load) in MSM, women who reported anal sex and women who did not report anal sex, including load detection threshold, geometrical mean and mean difference between groups tested by univariate linear regression analyses

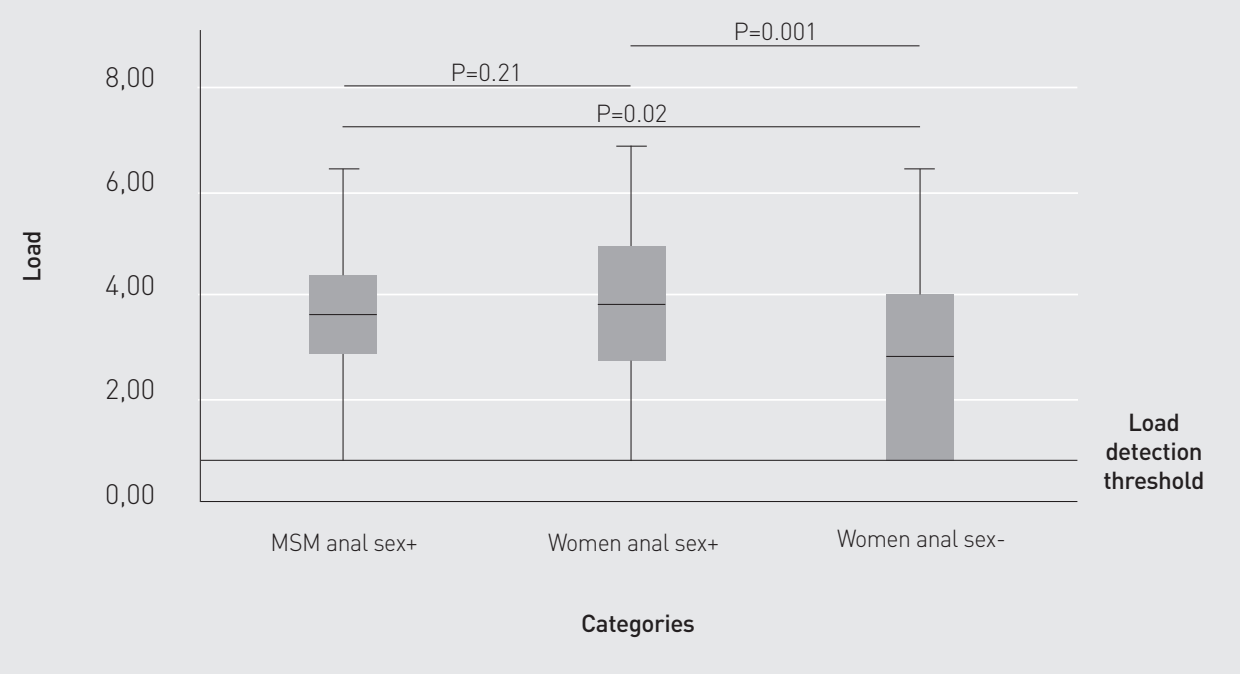


Among detectable load values, MSM who reported anal sex had a similar mean log anorectal chlamydia load (3.74) as women who reported anal sex (4.13, $\mathrm{P}=0.09$ ). Women who did not report anal sex (3.53) had lower mean log anorectal chlamydia load compared to women who did report anal sex $(4.14, \mathrm{P}=0.04)$ among detectable load values.

\section{Determinants associated with anorectal load}

None of the assessed determinants were significantly associated with anorectal load. Although in multivariable analyses adjusted for proctitis and age, antibiotic use in the past month was marginally significantly associated with anorectal chlamydia load. Individuals who used antibiotics in the past month had a 0.91 chlamydia/ml $95 \% \mathrm{Cl}-0.01-1.83, \mathrm{P}=0.05$ ) higher anorectal chlamydia load compared to individuals who did not use antibiotics in the past month. None of the assessed determinants were associated with high load (i.e. the upper quartile versus the other quartiles).

\section{Discussion}

Anorectal chlamydial load was comparable between MSM and women who reported anal sex. Women who did not report anal sex had a lower anorectal load, but within a comparable range to the other groups. Having anorectal symptoms was not associated with anorectal load in either MSM or women.

Anorectal chlamydia is known to be equally prevalent in each of the three groups $[9,17]$. We found an equal load in MSM and women who reported anal sex and no association with symptoms. This may suggest that the direct clinical impact (in terms of symptoms) and the public health impact (in terms of transmission potential) was similar between the two groups. In MSM and women reporting anal sex, chlamydia detection is at least in part caused by transmission due to anal sex. It is likely that transmission by anal sex causes the difference in mean load between women who did and did not report anal sex. Yet, there may be different causes for detection of anorectal chlamydia that affect the potential for further spread.

The anorectal chlamydia load in women who did not report anal sex remains unexplained. In these women, the mean load was lower, yet load values were in the same ranges as for MSM or women who reported anal sex. Moreover, the load differed with log 1 between women who did or did not report anal sex, which is lower than the mean load in women who did not report anal sex (log 2.8). This indicates that transmission routes other than anal sex could lead to substantial anorectal chlamydia load (range 1.84-6.31) in women. Previous studies suggest that autoinoculation (self infection) through vaginal secretions [15,29-31], concurrent transmission during sex [9] or infection via the intestinal canal[32,33] could lead to microbiological detection in 
women. After oropharyngeal chlamydia infection, the intestinal canal could serve as a reservoir that allows chlamydiae to persist indefinitely by immune down-regulation in the gut[32,33]. However, no studies in humans have confirmed any of these theories. Nevertheless, autoinoculation seems a very likely explanation for the substantial load because of anatomical proximity. Moreover, the majority (up to $95 \%$ ) of women with an anorectal infection have a concurrent urogenital infection $[8,9,18]$.

In contrast, the majority of MSM with an anorectal infection have a rectal only infection $[10,12]$ (i.e. without urogenital infection). Unfortunately MSM who did not report anal sex were not included in this study. It would be interesting to further study whether load differs according to anal sexual behaviour in MSM.

Since the sexual history asked about behaviour in the past six months, the women could have had anal sex with a chlamydia positive partner more than six months prior to consultation. Thereby, the recall period may be too short and the sexual history might not always be trustworthy. Possibly, MSM and women may have incorrectly reported whether or not they have had anal sex.

Contamination during sampling, although unlikely, cannot be completely ruled out. However, contamination by inadequate swab handling is unlikely to play a role since self-collected rectal samples are as valid for testing as provider-collected swabs [3,4]. Environmental contamination is unlikely since laboratory quality procedures reveal that positive samples do not cluster (data not shown). Women who did not report anal sex had a higher proportion of unquantifiable load samples compared to MSM and women who report anal sex. This finding concurs with the overall lower detectable load in this group. It is not surprising that these unquantifiable load samples are tested positive with NAAT, but have a low load below detection level as the quantitative PCR targets a single-copy gene and the NAAT a multi-copy gene. We cannot completely rule out false positives among these unquantifiable load samples. However, this would be very unlikely as routine CE certified NAATs with high sensitivity and specificity were used for diagnostics. Moreover, there is no reason to assume this would be different for MSM and women.

Anal symptoms were not associated with anorectal load for either MSM or women. Other determinants tested (for example age, nationality, number of sex partners or concurrent urogenital chlamydial were not associated with anorectal load in MSM or women. A study by Twin et al. also concluded that there were no associations between anorectal chlamydia load and prior chlamydia infection, symptoms or any demographic information [22]. This makes it difficult to target individuals with high load in care based on symptoms [15]. Targeting individuals with a high load based on the report of anal sex may be an option. Yet it has limitations, as half of all anorectal 
infections in MSM and women would be missed based on previous studies[34]. Moreover, women who did not report anal sex also have high anorectal chlamydia load (up to 6.31). Nevertheless, the extent to which chlamydia load reflects transmission potential or further consequences for morbidity (for example reproductive health in women) remains to be determined.

A systematic review of the epidemiology of organism load in genital chlamydia infection by Vodstrcil et al. revealed that load varies by specimen type and site of sampling specimens. Culture studies were more likely to have found an association between load and symptoms than NAAT studies [16]. This could indicate that viable organisms, such as those measured by culture, may be more relevant than total load, which is measured by NAAT. It is unknown whether this would be any different between MSM and women. This makes chlamydia viability an important future research area[16].

More research is needed to gain insight into the actual transmission of chlamydia, especially at anorectal sites. Given the lack of better data based on innovative laboratory measurement and transmission studies, and considering the similar load in MSM and women - whatever the reasons for detection may be - we are likely looking at real infections with potential clinical and transmission impacts. State-of-the-art practice acknowledges that anorectal chlamydia in MSM is relevant in terms of testing and treatment. This study shows that anorectal infections in women who report anal sex are of equal relevance, as chlamydia load values are comparable between MSM and women. Anorectal infections are contributing to the growing number of observed chlamydia cases in MSM and, according to recent studies, also in women $[8,9,15]$. Although the exact impact of load is yet unclear, testing and treatment of anorectal infections seems to remain important to preventing further spread and complications.

This study had several limitations. For practical reasons, a convenience sample was used in this study. The individuals included were, on average, younger than excluded individuals, precluding analyses in older individuals. There is some evidence to suggest that organism load in urogenital samples is higher in young individuals than older individuals[16]. MSM were on average older compared to women, which could have influenced load values. Nevertheless, anorectal chlamydia load was not associated with age in our current study, so we expect bias to be minimal. In addition, current data were limited since women with a rectal-only infection and MSM who did not report anal sex were not included in this study. This makes it difficult to generalise results to all MSM and women. Moreover, anal sex in MSM could not be stratified into receptive and insertive anal sex, possibly leading to the misclassification of receptive anal sex. Although the overall number of partners was recorded, there were no data available on the frequency of anal sex among MSM; exposure may have been higher, leading to a possible overestimation of the anorectal load in MSM. More research is needed to compare anorectal chlamydia load in MSM with and without receptive anal sex, and women with and without concurrent infection.

A general limitation encountered in chlamydia load studies is the lack of knowledge about the 
duration and load curve of a chlamydia infection. Differences in load may be due to different sampling moments during infection[35]. Possibly uncaptured bacterial factors (i.e. chlamydia genotype, local microbiome and co-infections like candida or HPV) or host factors (i.e. local immunity and microbiome) could explain variations in chlamydia load and it is unknown how they affected the findings. Animal models could be useful to obtain more insight in replication dynamics[35], although it is unclear whether these mechanisms are comparable to humans. Samples with a high bacterial load may be presumed to be sampled at the height of infection, while lower loads may indicate the beginning or resolution of a chlamydia infection[28]. Persistent chronic infections, with a low load which remains stable over a long period of time, are also suggested in literature[36,37].

Some recommend standardisation of organism load measures by correcting for the number of human cells present to account for sampling variability[16]. Counterarguments have put forward that commonly used human targets such as HLA-, beta-globin- or beta-actin genes are too broad a target for human cell quantification via PCR. They are present in all cells (for example columnar, squamous and inflammatory cells), not just in columnar cells, which is the cell type preferred by chlamydia[38]. It is to question whether normalisation of chlamydia by total human cell count provides relevant information about the chlamydia per epithelial cell concentration [39]. Caution must be used in samples with high inflammatory cell counts, as normalisation will result in low chlamydia/cell loads. This is important in studies correlating clinical features to the chlamydia load, as any positive relation might be masked using such an artificial low load. In this study we did not correct for human cells, i.e. HLA, as we cannot differentiate between leukocytes and epithelial cells with our PCR-assay. It is unlikely that this is a limitation to our study as results remained similar when we corrected for human cells (data not shown).

\section{Conclusions}

In conclusion, anorectal chlamydia infections in women who reported anal sex have similar bacterial loads as anorectal infections in MSM. This may imply similar transmission potential and clinical relevance. More research on anorectal chlamydia load is needed (for example viability and its role in transmission potential, and the development of sequelae).

\section{Acknowledgements}

We would like to thank Susanne Bogers, Lisette Houben and Mayk Lucchesi for technical assistance and the staff of the South Limburg STI clinic for their valuable contribution to data collection. 


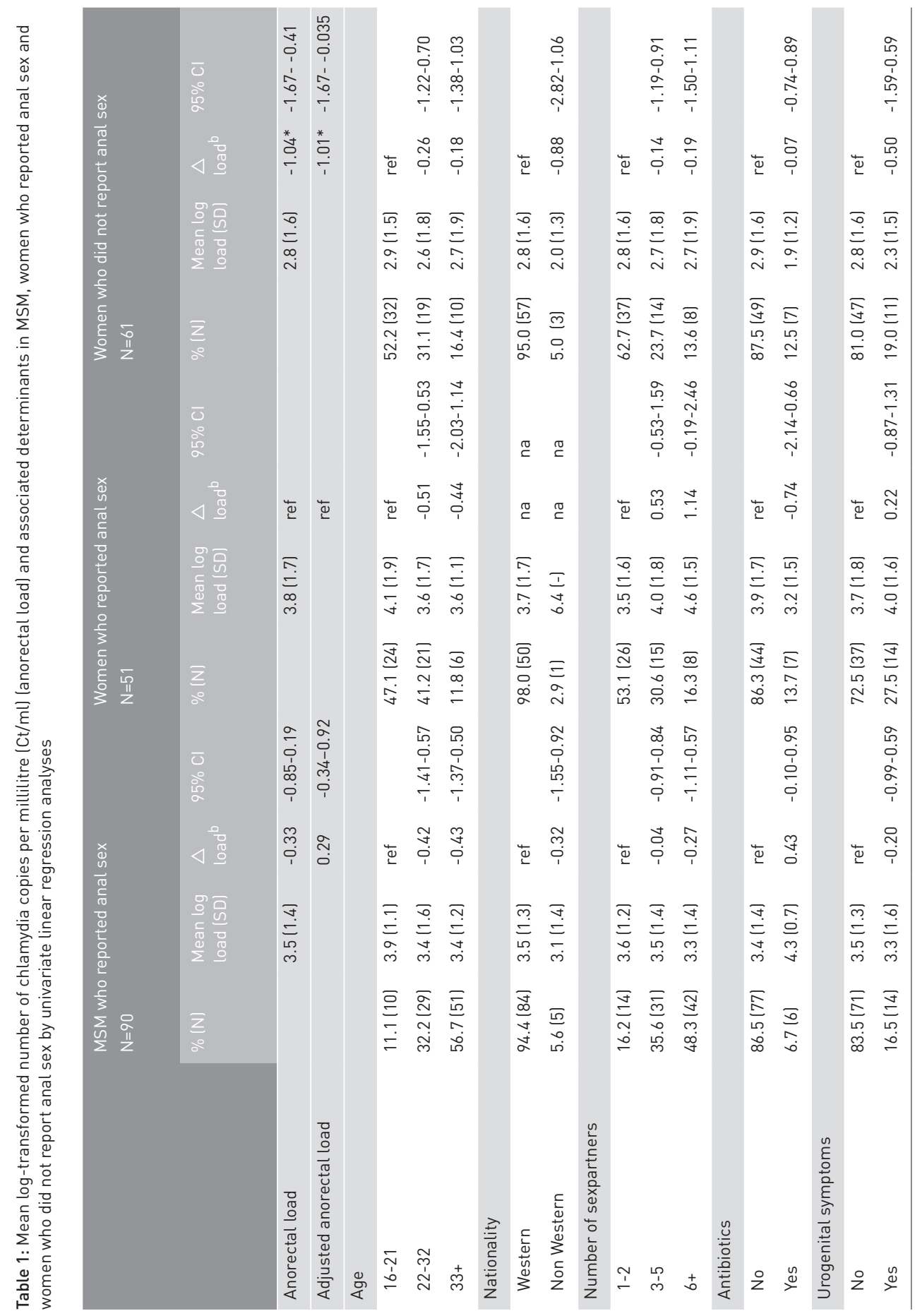




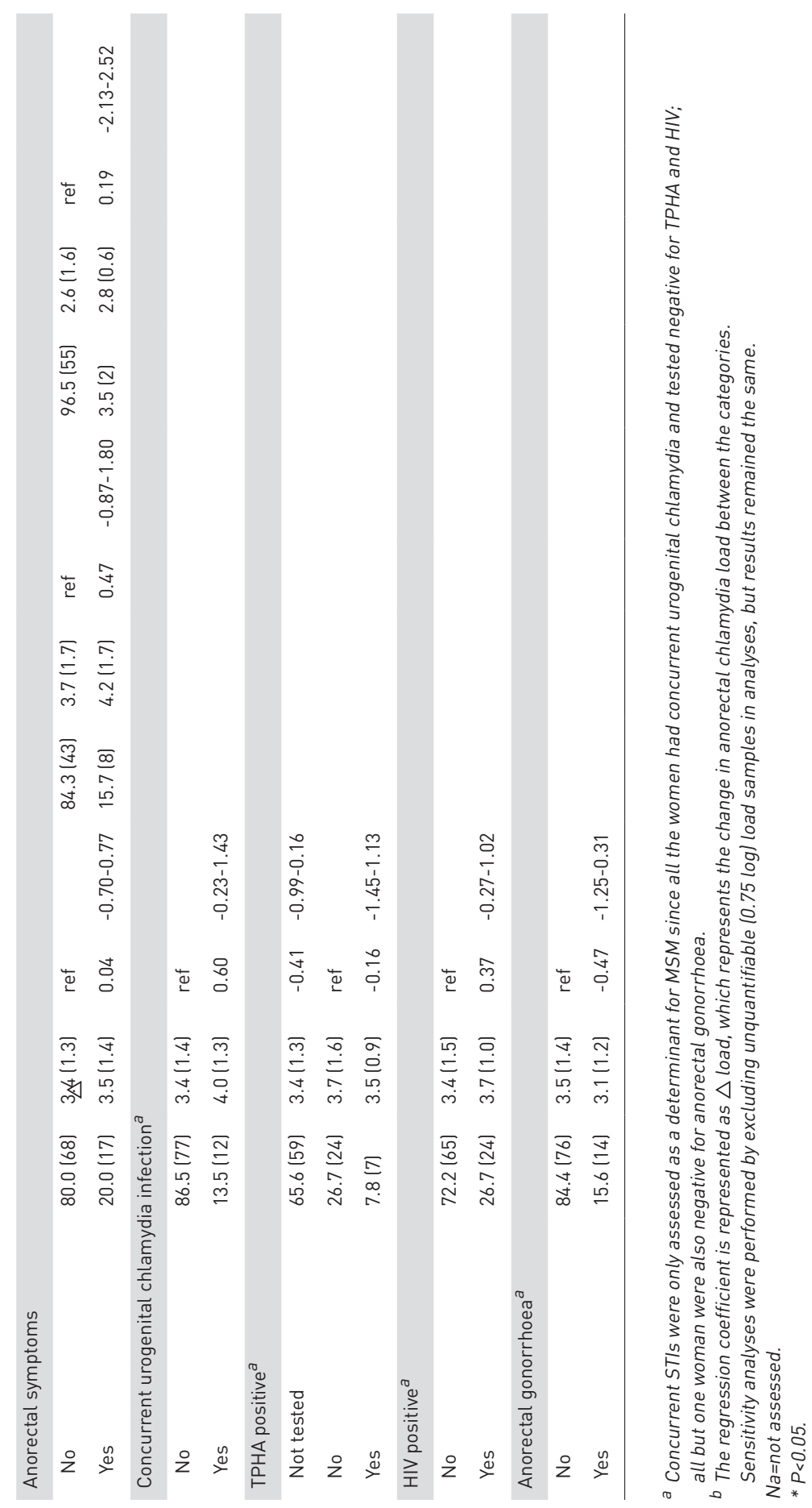




\section{References}

1. John R. Papp JS, Charlotte A. Gaydos, Barbara Van Der Pol. Recommendations for the Laboratory-Based Detection of Chlamydia trachomatis and Neisseria gonorrhoeae - 2014.

2. Schachter J, Philip SS. Testing men who have sex with men for urethral infection with Chlamydia trachomatis and Neisseria gonorrhoeae is only half the job, and we need the right tools. Sex Transm Dis. 2011;38: 925-927.

3. van der Helm JJ, Hoebe CJ, van Rooijen MS, Brouwers EE, Fennema HS, Thiesbrummel $H F$, et al. High performance and acceptability of self-collected rectal swabs for diagnosis of Chlamydia trachomatis and Neisseria gonorrhoeae in men who have sex with men and women. Sex Transm Dis. 2009;36: 493-497.

4. Moncada J, Schachter J, Liska S, Shayevich C, Klausner JD. Evaluation of self-collected glans and rectal swabs from men who have sex with men for detection of Chlamydia trachomatis and Neisseria gonorrhoeae by use of nucleic acid amplification tests. J Clin Microbiol. 2009;47: 1657-1662.

5. Workowski KA, Berman SM. Sexually transmitted diseases treatment guidelines, 2006. MMWR Recomm Rep. 2006;55: 1-94.

6. 2006 UK National Guideline for the Management of Genital Tract Infection with Chlamydia trachomatis. http://www.bashh.org/ documents/65.pdf.

7. Barry PM, Kent CK, Philip SS, Klausner JD. Results of a program to test women for rectal chlamydia and gonorrhea. Obstet Gynecol. 2010;115: 753-759.

8. Javanbakht M, Gorbach P, Stirland A, Chien M, Kerndt P, Guerry S. Prevalence and correlates of rectal Chlamydia and gonorrhea among female clients at sexually transmitted disease clinics. Sex Transm Dis. 2012;39: 917-922.

9. van Liere GA, Hoebe CJ, Wolffs PF, DukersMuijrers NH. High co-occurrence of anorectal chlamydia with urogenital chlamydia in women visiting an STI clinic revealed by routine universal testing in an observational study; a recommendation towards a better anorectal chlamydia control in women. BMC Infect Dis. 2014;14: 274.

10. Kent CK, Chaw JK, Wong W, al e. Prevalence of rectal, urethral, and pharyngeal chlamydia and gonorrhea detected in 2 clinical settings among men who have sex with men: San Francisco, California, 2003. Clin Infect Dis. 2005;41: 67-74.

11. van Liere GA, Hoebe CJ, Dukers-Muijrers NH. Evaluation of the anatomical site distribution of chlamydia and gonorrhoea in men who have sex with men and in high-risk women by routine testing: cross-sectional study revealing missed opportunities for treatment strategies. Sex Transm Infect. 2014;90: 58-60.

12. Patton ME, Kidd S, Llata E, Stenger M, Braxton J, Asbel L, et al. Extragenital gonorrhea and chlamydia testing and infection among men who have sex with men--STD Surveillance Network, United States, 2010-2012. Clin Infect Dis. 2014;58: 1564-1570.

13. Cachay ER, Sitapati A, Caperna J, Freeborn K, Lonergan JT, Jocson E, et al. Denial of risk behavior does not exclude asymptomatic anorectal sexually transmitted infection in HIV-infected men. PLoS One. 2009;4: e8504. 
14. Sexual attitudes and lifestyles in Britain: Highlights from Natsal-3 from. Available: http://www.natsal.ac.uk/media/2102/ natsal-infographic.pdf.

15. Ding A, Challenor R. Rectal Chlamydia in heterosexual women: more questions than answers. Int J STD AIDS. 2013;25: 587-592.

16. Vodstrcil LA, Mclver R, Huston WM, Tabrizi SN, Timms P, Hocking JS. The epidemiology of organism load in genital Chlamydia trachomatis infection - a systematic review. J Infect Dis. 2014;211: 1628-1645.

17. Peters RP, Dubbink JH, van der Eem L, Verweij SP, Bos ML, Ouburg S, et al.Crosssectional study of genital, rectal, and pharyngeal Chlamydia and gonorrhea in women in rural South Africa. Sex Transm Dis. 2014;41: 564-569.

18. Bax CJ, Quint KD, Peters RP, Ouburg S, Oostvogel PM, Mutsaers JA, et al. Analyses of multiple-site and concurrent Chlamydia trachomatis serovar infections, and serovar tissue tropism for urogenital versus rectal specimens in male and female patients. Sex Transm Infect. 2011;87: 503-507.

19. Koedijk FD, van Bergen JE, Dukers-Muijrers $\mathrm{NH}$, van Leeuwen AP, Hoebe CJ, van der Sande MA. The value of testing multiple anatomic sites for gonorrhoea and chlamydia in sexually transmitted infection centres in the Netherlands, 2006-2010. Int J STD AIDS. 2012;23: 626-631.

20. Wawer MJ, Gray RH, Sewankambo NK, Serwadda D, Li X, Laeyendecker O, et al. Rates of HIV-1 transmission per coital act, by stage of HIV-1 infection, in Rakai, Uganda. J Infect Dis. 2005;191:1403-1409.
21. Dukers-Muijrers NH, Speksnijder AG, Morre SA, Wolffs PF, van der Sande MA, Brink AA, et al. Detection of anorectal and cervicovaginal Chlamydia trachomatis infections following azithromycin treatment: prospective cohort study with multiple time-sequential measures of rRNA, DNA, quantitative load and symptoms. PLoS One. 2013;8: e81236.

22. Twin J, Moore EE, Garland SM, Stevens MP, Fairley CK, Donovan B, et al. Chlamydia trachomatis genotypes among men who have sex with men in Australia. Sex Transm Dis. 2011;38: 279-285.

23. Linssen CF, Jacobs JA, Stelma FF, van Mook WN, Terporten P, Vink C, et al. Herpes simplex virus load in bronchoalveolar lavage fluid is related to poor outcome in critically ill patients. Intensive Care Med. 2008;34: 2202-2209.

24. Vliegen I, Duijvestijn A, Stassen F, Bruggeman C. Murine cytomegalovirus infection directs macrophage differentiation into a pro-inflammatory immune phenotype: implications for atherogenesis. Microbes Infect. 2004;6: 1056-1062.

25. Jalal H, Stephen H, Curran MD, Burton J, Bradley M, Carne C. Development and validation of a rotor-gene real-time PCR assay for detection, identification, and quantification of Chlamydia trachomatis in a single reaction. J Clin Microbiol. 2006;44: 206-213.

26. van der Helm JJ, Sabajo LO, Grunberg AW, Morre SA, Speksnijder AG, de Vries HJ. Point-of-care test for detection of urogenital chlamydia in women shows low sensitivity. 
A performance evaluation study in two clinics in Suriname. PLoS One. 2012; 7: e32122.

27. Sambrook J, Fritsch E, Maniatis T (1989) Molecular cloning: a laboratory manual: Cold Spring Harbor Laboratory Press.

28. Dirks JA, Wolffs PF, Dukers-Muijrers NH, Brink AA, Speksnijder AG, Hoebe CJ.

Chlamydia trachomatis load in populationbased screening and STI-clinics: implications for screening policy. PLoS One. 2015;10: e0121433.

29. Barry PM, Kent CK, Philip SS, al e. Results of a program to test women for rectal chlamydia and gonorrhea. Obstet Gynecol. 2010;115: 753-759.

30. Hunte T, Alcaide M, Castro J. Rectal infections with chlamydia and gonorrhoea in women attending a multiethnic sexually transmitted diseases urban clinic. Int J STD AIDS 2010;21: 819-822.

31. Sethupathi M, Blackwell A, Davies H. Rectal Chlamydia trachomatis infection in women. Is it overlooked? Int J STD AIDS. 2010;21: 93-95.

32. Rank RG, Yeruva L. Hidden in plain sight: chlamydial gastrointestinal infection and its relevance to persistence in human genital infection. Infect Immun. 2014;82: 1362-1371.

33. $Y$ eruva L, Spencer N, Bowlin AK, Wang Y, Rank RG. Chlamydial infection of the gastrointestinal tract: a reservoir for persistent infection. Pathog Dis. 2013;68: 88-95.

34. van Liere GA, Hoebe CJ, Niekamp AM, Koedijk FD, Dukers-Muijrers NH. Standard symptomand sexual history-based testing misses anorectal Chlamydia trachomatis and neisseria gonorrhoeae infections in swingers and men who have sex with men. Sex Transm Dis. 2013:40: 285-289.

35. Price MJ, Ades AE, Angelis DD, Welton NJ, Macleod J, Soldan K, et al. Mixture-ofexponentials models to explain heterogeneity in studies of the duration of Chlamydia trachomatis infection. Stat Med. 2012;32: 1547-1560.

36. Caldwell HD, Wood H, Crane D, Bailey R, in Chlamydia trachomatis tryptophan synthase genes differentiate between genital and ocular isolates. J Clin Invest. 2003;111: 1757-1769.

37. Hogan RJ, Mathews SA, Mukhopadhyay S, Summersgill JT, Timms P. Chlamydial persistence: beyond the biphasic paradigm. Infect Immun. 2004;72: 1843-1855.

38. Moorman DR, Sixbey JW, Wyrick PB. Interaction of Chlamydia trachomatis with human genital epithelium in culture. J Gen Microbiol. 1986;132: 1055-1067.

39. Jalal H, Verlander NQ, Kumar N, Bentley $N$, Carne C, Sonnex C. Genital chlamydial infection: association between clinical features, organism genotype and load. J Med Microbiol. 2011;60: 881-888. 



$$
\begin{gathered}
\text { General } \\
\text { discussion }
\end{gathered}
$$


Chlamydia trachomatis (CT) is a major public health problem. Nevertheless, it remains a continuous challenge to control its spread and the spread of other sexually transmitted infections (STIS) such as Neisseria gonnorhoeae (NG). In this thesis, current testing practices and guidelines were evaluated to provide recommendations for future policy. We evaluated both national and international guidelines to obtain a broad perspective. Guidelines mainly focus on urogenital infections, especially in women, lacking focus on extra-genital testing. Therefore, a specific subject in this evaluation was extra-genital testing.

\section{Prevalence and missed infections with current testing guidelines}

Studies show anorectal CT/NG prevalence is substantial in MSM (1-18\%)[1-10], as well as in women $(7-17 \%)[1,6,10-26]$. Current guidelines recommend selective symptom and sexual history based anorectal testing as standard operating procedure in STI clinics. In chapter 4 and 6 we evaluated this selective anorectal testing by routine universal anorectal screening, irrespective of reported risk behaviour (anal sex) or symptoms. This first study consisted of MSM and swingers, as they are a risk group for (extra-genital) STI. The second study consisted of women in general who visited the STI clinic. Prevalence of anorectal STI was substantial with $7 \%-12 \%$ in MSM and women. In heterosexual male swingers the prevalence of anorectal STI was low (1.7\%). The sensitivity of selective testing to detect anorectal infections was low $40 \%-71 \%$ in all groups. Over half of anorectal CT and NG infections would be missed using current selective testing in MSM, swingers and women in general.

\section{Possible routes of transmission}

A possible explanation for these missed infections could be underreporting of anal sex. However, this reporting bias is unlikely to explain a major part as then such bias would need to be unrealistically high and consistent across studies and countries. Bias by using a recall period that is too short to capture the behavioral exposure is probably more likely, given that anal sex is usually recorded as behavior in a given time period (usually a couple of months). Anal sex is commonly reported by women and MSM[1,6,9,10,12-27]. Infections may have been acquired before the beginning of the screening interval, and be unnoticed due to its asymptomatic nature and lack of extra-genital testing. It has been suggested that anorectal pathogens may potentially be transmitted by practices that involve contact with the anus other than penetrative anal-genital sex (for example, by transmission by fingers or by sex toys). The anal use of fingers and sex toys was reported frequently among women (20\% and $9 \%$ respectively), but it was not associated with anorectal CT as described in chapter 6. Another study found an association between the anal use fingers/sex toys and anorectal STI[28]. Altogether, evidence for this is inconclusive, and further studies are recommended to get more insight in alternative transmission routes such as fingers and sex toys. 
A different theory to explain anorectal CT detection involves the gastro-intestinal (GI) tract as a reservoir for $\mathrm{CT}$ detection. While asymptomatic $\mathrm{CT}$ infections have been detected in the $\mathrm{Gl}$ tract of neonates exposed at birth, and rectal shedding has been observed in children in trachoma endemic areas, the theory that the GI tract could act as a reservoir in humans, was more recently shaped by new observations in mice[29,30]. After oropharyngeal exposure to CT, the bacterium can survive in the GI tract due to down regulation of the immune system. However, evidence to support long term persistence of $\mathrm{CT}$ in the $\mathrm{GI}$ tract in human adults is lacking.

Contamination of the anorectal swab due to inadequate swab handling (cross-contamination of genital infection) or by environmental exposure cannot be entirely ruled out[10,27]. However, it is very unlikely that anorectal detection is caused by contamination[31] since sampling instructions were clear. Trained study nurses provided women with a visual diagram and verbal instructions about how to take separate self-collected vaginal and rectal swabs. The patient was instructed to insert the swab $2.5 \mathrm{~cm}$ into the vagina/anus, rotate it for 5 to 10 seconds, and then place it in a capped tube to avoid potential contamination. Moreover, positive samples did not cluster, which makes environmental exposure during the laboratory analyses unlikely.

\section{Anatomical site distribution}

To obtain more insight in these anorectal infections, chapter $\mathbf{5}$ assessed the anatomic site distribution of CT and NG in both MSM and women. The majority of women with an anorectal infection has a concurrent urogenital infection (up to 95\%). On the contrary, the majority of MSM with an anorectal infection had a rectal-only infection, that is, without concurrent urogenital infection (up to $86 \%$ ). These results are conclusive with other studies; $83 \%$ to $90 \%$ concurrent infections in women[11,18,23,32] and 70\% to 91\% rectal-only anorectal infections in MSM[3,5,8,9]. Possibly MSM more often have receptive anal sex compared to women. However, in our studies from chapters 4 and $\mathbf{6}$ it appeared that MSM and women report anal sex about as often $131 \%$ versus $53 \%$ ). Other studies also concluded comparable rates of reported anal sex in both groups of $4 \%$ to $100 \%[1,6,9,10,12-27]$. Moreover, the proportion of anorectal infections missed is comparable in women (47-71\%) and MSM (40-52\%), as is described in chapters 4 and 6 . Therefore a difference in reporting bias between MSM and women is expected to be minimal.

The most plausible reason for the difference in anatomic site distribution is autoinoculation of vaginal secretions in women. Chapter 6 elucidates on this by showing that $95 \%$ of women with anorectal CT have concurrent urogenital CT, and $75 \%$ of women with urogenital CT have concurrent anorectal CT. The anatomical proximity of genital and anorectal site makes this plausible, but this is not yet confirmed by rigorous data.

\section{Treatment}

In case treatment regimens are equal for urogenital and anorectal infections, concurrent anorectal infections would be automatically treated when a urogenital CT infection is diagnosed. In the Nether- 
lands, Australia and the UK a 7 day course for doxycycline is recommended for anorectal CT and a single dose azithromycin is recommended for urogenital CT[33-35]. In the US both therapies are considered equal first line treatments for anorectal CT[36]. There is current debate in literature; some studies conclude that azithromycin is a suboptimal treatment for anorectal CT[37-40], while others found both treatments equally effective[41]. A double blind RCT could provide answers to this treatment debate.

\section{Rectal-only infections}

In light of this treatment debate, it is important to know who has a rectal-only infection and who has a concurrent urogenital anorectal infection. In case concurrent anorectal infections are coincidentally treated with a urogenital infection, rectal-only infections would not be treated as they are not routinely tested for. As rectal-only infections are prevalent in both MSM and women, chapter $\mathbf{7}$ assesses factors associated with rectal-only CT and NG infection in both groups. Only few factors were found to be associated with rectal-only infections; for MSM, not being a sex worker, exclusively having sex with men and absence of urogenital symptoms for rectal-only CT. An other study also found that the absence of urogenital symptoms was associated with a rectalonly CT infection in MSM[42]. Factors associated with rectal-only NG in MSM were having been warned by an (ex) partner, having anoropharyngeal NG infection, and absence of urogenital symptoms. For women older age and non-Western nationality were associated with rectal-only CT. No factors were found to be associated with rectal-only NG in women which was in line with a study by Trebach et al[42]. Previous STI testing in the past two years was not associated with rectal-only infections. Therefore rectal-only CT/NG infections do not seem to cluster within individuals. Overall, only a few factors were associated with rectal-only infections in both MSM and women. This makes anorectal screening algorithms based on a priori patient characteristics challenging, because of low discriminatory power.

\section{Anorectal load}

As shown in the study from chapter 7, prevalence of anorectal CT is comparable between MSM and women, but the anatomic site distribution of CT/NG infections and factors associated with rectal-only CT/NG infections differ between the two groups. This may give rise to questioning whether anorectal infections in MSM and women detected by a NAAT are comparable in clinical impact and transmission potential. Chapter 8 assesses the bacterial load of anorectal CT infections in (1) MSM who reported anal sex las recommended in STI guidelines[36], and women with concurrent urogenital chlamydia who (2) reported anal sex and (3) did not report anal sex. The clinical impact may be considered in terms of symptoms. Anal symptoms were equally reported between MSM and women with CT who reported anal sex (16\%-20\%) and this is consistent with other studies[22]. Moreover, anal symptoms and other determinants were not associated with load. Therefore, we might conclude that the clinical impact of anorectal STI is comparable 
between MSM and women. However, this only applies to non-LGV anorectal infections. In women, LGV is very unusual, although systematic assessment of LGV in women is scarce[43].

Transmission potential could be carefully considered in terms of bacterial load (for example high loads indicate a higher transmission potential for viral STIs[44]). The mean anorectal chlamydia load was similar for MSM and women who reported having anal sex, indicating anorectal infections in these groups are of equal relevance. Anorectal chlamydia load was significantly higher in the latter two groups than in women who did not report having anal sex. This may imply autoinoculation as a possible transmission route, explaining the lower load detected in women who did not report having anal sex. However, detectable load values had a similar range in all three groups. This shows that both high and low loads were found women who did not report anal sex. This means that we should consider these positive anal tests as real infections as we do in MSM.

\section{Implications for practice and recommendations}

Anorectal STI are contributing to the growing number of observed chlamydia cases in MSM and, according to recent studies, also in women[14,18,45]. Therefore testing and treatment of anorectal STI remains important. Moreover, adequate treatment reduces or prevents the clinical impact of anorectal STI.

To improve extra-genital case finding, easy and acceptable testing methods are of great value. The acceptance of NAATs for extra-genital testing was the first step in this process. In 2014, the CDC published revised guidelines in which NAATs are also recommended for anorectal and oropharyngeal chlamydia testing. However, NAATs are not FDA-cleared for use with anorectal or oropharyngeal swab specimens[36]. The performance of nucleic acid amplification tests (NAATs) with respect to overall sensitivity, specificity, and ease of specimen transport is better than that of any of the other tests available for the diagnosis of chlamydial and gonococcal infections $[1,46]$. Additionally, extra-genital sampling sites should be clearly included in testing guidelines. High prevalence of anorectal STI, low sensitivity of current selective testing and high rates of rectalonly support routine universal anorectal screening in MSM. Especially, given the lack of useful indicators for rectal-only infections, selective screening based on a priori patient characteristics will have low discriminatory power in MSM. Additionally, the occurrence of LGV infections in MSM support this active approach. In the UK, this active approach is already advised; the 2014 BASHH guidelines for asymptomatic MSM were revised; all anatomical sites should be sampled in MSM (urethra, pharynx and rectum), regardless of reported behaviour. In addition, self taken samples from the urethra, pharynx and rectum are acceptable alternatives to clinician taken samples[34]. On the contrary, in the US, selective testing based on reported receptive anal intercourse is advised[36].

Supporting evidence is lacking on extra-genital testing in heterosexual males. Although sensitivity of selective testing was low for anorectal STI in this group, the prevalence of anorectal STI was also low. 
In women, routine universal anorectal screening could be supported by the substantial prevalence of anorectal CT. However, costs might be a problem. Moreover, women have high shares of concurrent anorectal-urogenital infections. Up till now it is not clear whether these concurrent anorectal infections are coincidentally treated with the urogenital infection. However, NAATs detect anorectal STI in women and women also have high anorectal CT loads, even when anal sex was not reported. Theoretically, these anorectal infections in women could lead to transmission between individuals, and possibly also within an individual, causing reproductive health problems. Therefore it is important to treat anorectal STI in women. By restricting anorectal testing to women with genital infections, concurrent anorectal infections would be diagnosed. A more cost-effective strategy to achieve this, without having to ask the women to come back for anorectal testing, could be to directly take an anorectal sample at the routine genital STI screening visit, but to only do further anorectal laboratory testing only on women who tested positive for their genital infection in the laboratory. In such case, fewer anorectal laboratory tests would have to be performed compared to routine screening of women, perhaps reducing costs. Yet, such phased testing strategy would also demand more complex laboratory logistics, increase time to return test results. Also, this strategy would also leave out the rectal-only infections that remain untested and unmanaged. Rectal-only CT infections are hard to target in practice given the lack of useful indicators to guide selective screening.

Restrictions in financial means for anorectal testing has led some clinicians to taking a pragmatic approach by directly treating genital CT positive women with doxycycline, assuming this will treat a potential concurrent anorectal infection better than when using azithromycin. Still, the strategy to use doxycycline in anorectal CT is not based on solid evidence and is limited by the higher non-compliance rates seen with doxycycline use[47]. Until we better understand the effectiveness in clinical daily practice of anorectal CT treatment regimens, the impact of not testing anorectal CT in women is difficult to assess. Well designed randomized controlled trials on this topic are needed.

\section{Key messages}

Anorectal CT and NG could form a high potential for avoidable transmission and to some extent for avoidable morbidity in MSM and in women. Anorectal CT and NG can be successfully detected using NAAT on simple self-taken samples and both men and women are willing to test. Strategies to increase the reach of these self-taken samples, and thereby lower barriers to accessing STI care for both patients and providers. More studies are needed for strategies to increase the reach of these self-taken samples, and thereby lower barriers to accessing STI care for both patients and providers, including the use of internet-based programs, e-health strategies and home-collection. 
Testing should not solely be guided by symptoms or behavioural exposure as the large majority of anorectal STI are asymptomatic and over half of the anorectal infections occur in the absence of (the patient reporting) such exposures in both MSM and women. The majority of anorectal infections in MSM are rectal-only nfections that are not being coincidentally treated by managing urogenital infections. Lacking better data to guide testing, routine anorectal CT and NG testing is warranted in MSM. In women, a more restricted testing policy could be preferred. As the majority of women with an anorectal infection have a concurrent urogenital infection this could be (1) anorectal testing in women with genital infections, or (2) directly treating urogenital CT positive women with doxycycline.

\section{Knowledge gaps for future research}

To date, there is no evidence that the clinical impact and the public health impact, in terms of symptoms and transmission, of anorectal infections is any different for women than it is for MSM. To fully understand the impact of anorectal infections, future studies should address extra-genital and genital morbidity caused by extra-genital infections. Randomized controlled trials are needed to determine whether treatment efficacy for extra-genital infections differs from that of genital infections. The full spectrum including potential further transmission of extra-genital infections to genital sites and related morbidity has not yet been evaluated in women. Prospective studies in couples or modelling studies could reveal more insight on the transmission probabilities between partners and between anatomic sites, and the associated factors. Morbidity and transmission may depend on the extent the bacteria is able to replicate or presents in high loads, which is unknown in MSM and in women. Studies using appropriate laboratory techniques, such as quantitative PCR for measuring bacterial load, and culture to detect viable bacteria and antibiotic resistance, to address these aspects are needed. These will greatly aid our understanding of extra-genital infections and the differences presented by these infections in MSM and women.

Finally, cost-effectiveness of extra-genital testing has been evaluated for HIV infected MSM and was shown cost-effective in case of anorectal CT and NG. While it has been suggested that the lower prevalence of NG in women overall makes it more costly to screen for both CT and NG in women compared to MSM, this is in practice not likely a valid argument as most NAATs for CT are duplex assays also measuring NG with the same costs. Hidden anorectal infections might fuel the transmission of CT and NG and thereby increase the overall STI burden. This may be very important in the context of HIV and anorectal infection.

STI care providers should be aware of which (sub)groups they need to test more often and the common occurrence of extra-genital infections. Ideally, STI care providers give care providers knowledge on where to find certain expertise, such as on anorectal testing. This would enhance integrating the services between STI care providers in a region to optimise CT test practice and improve the cooperation between STI care providers. 


\section{References}

1. Bachmann LH, Johnson RE, Cheng $H$, Markowitz L, Papp JR, Palella FJ, Jr., et al. Nucleic acid amplification tests for diagnosis of Neisseria gonorrhoeae and Chlamydia trachomatis rectal infections. J Clin Microbiol. 2010;48: 1827-1832.

2. Chow EP, Tomnay J, Fehler G, Whiley D, Read TR, Denham I, et al. Substantial increases in chlamydia and gonorrhea positivity unexplained by changes in individual-level sexual behaviors among men who have sex with men in an Australian sexual health service from 2007 to 2013. Sex Transm Dis. 2015;42: 81-87.

3. Dudareva-Vizule S, Haar K, Sailer A, Wisplinghoff $\mathrm{H}$, Wisplinghoff $F$, Marcus U. Prevalence of pharyngeal and rectal Chlamydia trachomatis and Neisseria gonorrhoeae infections among men who have sex with men in Germany. Sex Transm Infect. 2013;90: 46-51.

4. Ivens D, Macdonald K, Bansi L, Nori A. Screening for rectal chlamydia infection in a genitourinary medicine clinic. Int J STD AIDS. 2007; 18: 404-406.

5. Kent CK, Chaw JK, Wong W, Liska S, Gibson S, Hubbard G, et al. Prevalence of rectal, urethral, and pharyngeal chlamydia and gonorrhea detected in 2 clinical settings among men who have sex with men: San Francisco, California, 2003. Clin Infect Dis. 2005;41: 67-74.

6. Koedijk FD, van Bergen JE, Dukers-Muijrers $\mathrm{NH}$, van Leeuwen AP, Hoebe CJ, van der Sande MA. The value of testing multiple anatomic sites for gonorrhoea and chlamydia in sexually transmitted infection centres in the Netherlands, 2006-2010. Int J STD AIDS. 2012;23: 626-631.

7. Marcus U, Ort J, Grenz M, Eckstein K, Wirtz K, Wille A. Risk factors for HIV and STI diagnosis in a community-based HIV/STI testing and counselling site for men having sex with men (MSM) in a large German city in 2011-2012. BMC Infect Dis. 2015;15: 14.

8. Patton ME, Kidd S, Llata E, Stenger M, Braxton J, Asbel L, et al. Extragenital gonorrhea and chlamydia testing and infection among men who have sex with men--STD Surveillance Network, United States, 2010-2012. Clin Infect Dis. 2014;58: 1564-1570.

9. Peters RP, Verweij SP, Nijsten N, Ouburg S, Mutsaers J, Jansen CL, et al. Evaluation of sexual history-based screening of anatomic sites for chlamydia trachomatis and neisseria gonorrhoeae infection in men having sex with men in routine practice. BMC Infect Dis. 2011;11: 203.

10. van der Helm JJ, Hoebe CJ, van Rooijen MS, Brouwers EE, Fennema HS, Thiesbrummel HF, et al. High performance and acceptability of self-collected rectal swabs for diagnosis of Chlamydia trachomatis and Neisseria gonorrhoeae in men who have sex with men and women. Sex Transm Dis. 2009;36: 493-497.

11. Barry PM, Kent CK, Philip SS, Klausner JD. Results of a program to test women for rectal chlamydia and gonorrhea. Obstet Gynecol. 2010;115: 753-759.

12. Bazan JA, Carr Reese P, Esber A, Lahey S, Ervin M, Davis JA, et al. High prevalence of rectal gonorrhea and Chlamydia infection in women attending a sexually transmitted disease 
clinic. J Womens Health (Larchmt). 2015;24: 182-189.

13. Cosentino LA, Campbell T, Jett A, Macio I, Zamborsky T, Cranston RD, et al. Use of nucleic acid amplification testing for diagnosis of anorectal sexually transmitted infections. J Clin Microbiol. 2012;50: 2005-2008.

14. Ding A, Challenor R. Rectal Chlamydia in heterosexual women: more questions than answers. Int J STD AIDS. 2013;25: 587-592.

15. Garner AL, Schembri G, Cullen T, Lee V. Should we screen heterosexuals for extra-genital chlamydial and gonococcal infections? Int J STD AIDS. 2014;26: 462-466.

16. Gratrix J, Singh AE, Bergman J, Egan C, Plitt SS, McGinnis J, et al. Evidence for increased Chlamydia case finding after the introduction of rectal screening among women attending 2 Canadian sexually transmitted infection clinics. Clin Infect Dis. 2014;60: 398-404.

17. Hunte T, Alcaide M, Castro J. Rectal infections with chlamydia and gonorrhoea in women attending a multiethnic sexually transmitted diseases urban clinic. Int J STD AIDS. 2011;21: 819-822.

18. Javanbakht $M$, Gorbach $P$, Stirland A, Chien $M$, Kerndt $P$, Guerry S. Prevalence and correlates of rectal Chlamydia and gonorrhea among female clients at sexually transmitted disease clinics. Sex Transm Dis. 2012;39: 917-922.

19. Ladd J, Hsieh YH, Barnes M, Quinn N, JettGoheen M, Gaydos CA. Female users of internet-based screening for rectal STIs: descriptive statistics and correlates of positivity. Sex Transm Infect. 2014;90: 485-490.
20. Musil K, Currie M, Sherley M, Martin S. Rectal chlamydia infection in women at high risk of chlamydia attending Canberra Sexual Health Centre. Int J STD AIDS. 2015.

21. Ostergaard L, Agner T, Krarup E, Johansen UB, Weismann K, Gutschik E. PCR for detection of Chlamydia trachomatis in endocervical, urethral, rectal, and pharyngeal swab samples obtained from patients attending an STD clinic. Genitourin Med. 1997; 73: 493-497.

22. Peters RP, Dubbink JH, van der Eem L, Verweij SP, Bos ML, Ouburg S, et al. Cross-sectional study of genital, rectal, and pharyngeal Chlamydia and gonorrhea in women in rural South Africa. Sex Transm Dis. 2014;41: 564-569.

23. Peters RP, Nijsten N, Mutsaers J, Jansen CL, Morre SA, van Leeuwen AP. Screening of oropharynx and anorectum increases prevalence of Chlamydia trachomatis and Neisseria gonorrhoeae infection in female STD clinic visitors. Sex Transm Dis. 2011;38: 783-787.

24. Sethupathi M, Blackwell A, Davies H. Rectal Chlamydia trachomatis infection in women. Is it overlooked? Int J STD AIDS. 2009;21: 93-95.

25. Shaw SG, Hassan-Ibrahim M, Soni S. Are we missing pharyngeal and rectal infections in women by not testing those who report oral and anal sex? Sex Transm Infect. 2013;89:397.

26. Trebach JD, Chaulk CP, Page KR, Tuddenham S, Ghanem KG. Neisseria gonorrhoeae and Chlamydia trachomatis among women reporting extragenital exposures. Sex Transm Dis. 2015;42: 233-239. 
27. Mercer $\mathrm{CH}$, Tanton $\mathrm{C}$, Prah $\mathrm{P}$, Erens B, Sonnenberg P, Clifton S, et al. Changes in sexual attitudes and lifestyles in Britain through the life course and over time: findings from the National Surveys of Sexual Attitudes and Lifestyles (Natsal). Lancet. 2013;382: 1781-1794.

28. de Vries HJ, Zingoni A, White JA, Ross JD, Kreuter A. 2013 European Guideline on the management of proctitis, proctocolitis and enteritis caused by sexually transmissible pathogens. Int J STD AIDS.25: 465-474.

29. Rank RG, Yeruva L. An alternative scenario to explain rectal positivity in Chlamydia-infected individuals. Clin Infect Dis. 2015;60: 1585-1586

30. Yeruva L, Spencer N, Bowlin AK, Wang Y, Rank RG. Chlamydial infection of the gastrointestinal tract: a reservoir for persistent infection. Pathog Dis. 2013;68: 88-95.

31. Chan SY, Jose S, King R, Pakianathan MR, Sabin C, Sadiq ST, et al. How likely is environmental or patient cross-contamination of Chlamydia trachomatis DNA to lead to false positive results in patients attending our clinic? Sex Transm Infect. 2012;89: 105-107.

32. Bax CJ, Quint KD, Peters RP, Ouburg S, Oostvogel PM, Mutsaers JA, et al. Analyses of multiple-site and concurrent Chlamydia trachomatis serovar infections, and serovar tissue tropism for urogenital versus rectal specimens in male and female patients. Sex Transm Infect. 2011;87: 503-507.

33. de Vries HJC, van Doornum GJJ, Bax CJ Multidisciplinaire Richtlijn Seksueel Overdraagbare Aandoeningen voor de 2e Lijn, Nederlandse Vereniging voor Dermatologie en Venereologie.

<http://www.tinyurl.soarichtlijn2012>

34. Ross J, Brady M, Clutterbuck D, Doyle T, Hart G, Hughes G, Nardone A, Patel R, Stuart D, Sullivan A, Steinberg P, Wilson J, Winter A. BASHH Recommendations for Testing for Sexually Transmitted Infections in Men who have Sex with Men </documents/BASHH\%20 Recommendations\%20for\%20testing\%20 for\%20STIs\%20in\%20MSM\%20-\%20FINALpdf>.

35. Australasian, Sexual Health Alliance. Australian STI Management Guidelines for Use in Primary Care. http://www.sti.guidelines.org.au/sexuallytransmissible-infections/chlamydia.

36. Workowski KA, Bolan GA. Sexually Transmitted Diseases Treatment Guidelines, 2015. Recommendations and Reports. 2015: 1-137.

37. Elgalib A, Alexander S, Tong CY, White JA. Seven days of doxycycline is an effective treatment for asymptomatic rectal Chlamydia trachomatis infection. Int J STD AIDS. 2011;22: 474-477.

38. Hathorn E, Opie C, Goold P. What is the appropriate treatment for the management of rectal Chlamydia trachomatis in men and women? Sex Transm Infect. 2012;88: 352-354.

39. Khosropour CM, Dombrowski JC, Barbee LA, Manhart LE, Golden MR. Comparing azithromycin and doxycycline for the treatment of rectal chlamydial infection: a retrospective cohort study. Sex Transm Dis. 2014;41: 79-85.

40. Steedman NM, McMillan A. Treatment of asymptomatic rectal Chlamydia trachomatis: is single-dose azithromycin effective? Int J STD AIDS. 2009;20: 16-18. 
41. Drummond F, Ryder N, Wand H, Guy R, Read P, McNulty AM, et al. Is azithromycin adequate treatment for asymptomatic rectal chlamydia? Int J STD AIDS. 2011;22: 478-480.

42. Gratrix J, Singh AE, Bergman J, Egan C, McGinnis J, Drews SJ, et al. Prevalence and characteristics of rectal chlamydia and gonorrhea cases among men who have sex with men after the introduction of nucleic acid amplification test screening at 2 Canadian sexually transmitted infection clinics. Sex Transm Dis. 2014;41: 589-591.

43. Heiligenberg M, Verweij SP, Speksnijder AG, Morre SA, de Vries HJ, Schim van der Loeff MF. No evidence for LGV transmission among heterosexuals in Amsterdam, the Netherlands. BMC Res Notes. 2014;7: 355.

44. Wawer MJ, Gray RH, Sewankambo NK, Serwadda D, Li X, Laeyendecker 0 , et al. Rates of HIV-1 transmission per coital act, by stage of HIV-1 infection, in Rakai, Uganda. J Infect Dis. 2005;191: 1403-1409.

45. van Liere GA, Hoebe CJ, Wolffs PF, DukersMuijrers NH. High co-occurrence of anorectal chlamydia with urogenital chlamydia in women visiting an STI clinic revealed by routine universal testing in an observational study; a recommendation towards a better anorectal chlamydia control in women. BMC Infect Dis. 2014;14: 274.

46. Schachter J, Moncada J, Liska S, Shayevich C, Klausner JD. Nucleic acid amplification tests in the diagnosis of chlamydial and gonococcal infections of the oropharynx and rectum in men who have sex with men. Sex Transm Dis. 2008;35: 637-642.
47. Odesanmi TY, Wasti SP, Odesanmi OS, Adegbola 0, Oguntuase 00, Mahmood S. Comparative effectiveness and acceptability of home-based and clinic-based sampling methods for sexually transmissible infections screening in females aged 14-50 years: a systematic review and meta-analysis. Sex Health. 2013;10: 559-569. 



\section{Summary}

Nederlandse samenvatting

Dankwoord

Curriculum Vitae

Portfolio

List of publications

Valorisation of the thesis 



$$
\text { Chapter } 10
$$

Summary 
The studies described in this thesis assessed the effectiveness of the current national and international procedures to control Chlamydia trachomatis (CT), including the current testing practices and guidelines, and provide recommendations for future policy and research.

\section{Chapter 2: CT screening to reach young people in addition to regular care}

Internet-based Chlamydia Screening was introduced in the Netherlands in 2008-2010 to detect and treat asymptomatic infections, and to limit ongoing transmission through annual testing and treatment of CT in young people (16-29 years). A key stone for an effective large scale screening programme is capturing substantial numbers of new, CT positive, individuals in addition to regular care such as the sexually transmitted infection (STI) clinic, GPs, and gynaecologists. By using data from all CT care providers in Eastern South Limburg from 2006 to 2010, we found that the majority $(81 \%, 4298 / 5352)$ of CT screening participants have not been previously tested by regular care. CT prevalence was similar in persons who were previously tested by regular care $(4.5 \%)$ and persons who were not previously tested by regular care (4.8\%). Thereby, population based CT screening adds to regular care by testing a hidden key population of young individuals hidden to current care.

\section{Chapter 3: CT testing within one geographical region}

Dutch STI care is organized in a similar way as in the UK and Australia, with a major role for public health care, ie, STI clinics, and general practitioners (GPS). STI clinics serve specific highrisk groups, including young people laged <25), while GPs serve a more general population. To obtain more insight in CT testing practices among young people, we evaluated and compared CT test practices of different STI care providers; STI clinics, GPs, gynaecologists and chlamydia screening in one geographical area (Eastern South Limburg). A total of 22.831 tests were performed and $8.2 \%$ were positive $(n=1868)$. Results show that STI clinics performed most CT tests in men, whereas GPs performed most CT tests in women. Gynaecologists perform a substantial proportion of CT testing in women, although GPs and STI clinics are mainly responsible for CT diagnosis. For all STI care providers, higher age (22-29 years )was associated with increased testing, but lower test positivity. Extra-genital CT testing is rarely performed outside the STI clinic and needs to be promoted, especially in men who have sex with men (MSM), because of the high prevalence of extra-genital STI. Evaluations such as provided here can help optimise CT test practice and could improve the cooperation between STI care providers, which is already in place in several countries.

\section{Chapter 4: Anorectal CT/NG infections missed in MSM and swingers}

MSM and swingers are an important risk group for STI including anorectal STI. Current guidelines advocate symptom- and sexual history-based anorectal testing (selective testing). In this study, all MSM and swingers ( $\mathrm{N}=1690)$ visiting the STI clinic were tested routinely universally at anorectal site 
to evaluate the sensitivity of selective anorectal testing. Prevalence of anorectal CT/NG by routine universal anorectal testing was $9 \% / 4 \%$ for homosexual MSM, 7\%/3\% for bisexual MSM, 4\%/1\% for bisexual male swingers, $1 \% / 0.3 \%$ for heterosexual male swingers, and $7 \% / 1 \%$ for female swingers. Sensitivity of selective testing varied between $40 \%-52 \%$ in the different risk groups. In conclusion, over half of anorectal CT and NG infections were missed in MSM and swingers using current selective symptom- and sexual history-based testing. Routine universal anorectal testing may be a more effective strategy for interrupting the ongoing transmission in high-risk sexual networks.

\section{Chapter 5: The anatomical site distribution of CT/NG in MSM and high-risk women}

Insight into the anatomical site distribution of STI is important to understand the appropriateness of current extra-genital control strategies, for example coincidental treatment with urogenital infections. We included MSM ( $=2436)$ and high-risk women (prostitutes and swingers, $\mathrm{N}=1321$ ) who were routinely universally tested for anorectal, oropharyngeal and urogenital CT and/or NG. Overall CT/NG prevalence was $10 \% / 6 \%$ in MSM and $7 \% / 3 \%$ in high-risk women. Rectal-only infections, that is, without concurrent urogenital infection, accounted up to $76 \%$ of all infections in MSM and 59\% in women. For concurrent infections this amounted to $14 \%$ for MSM and $54 \%$ for high-risk women. Therefore, testing only for urogenital infections is insufficient, as many infections would be overlooked. The use of coincidental treatment is a suboptimal control strategy for halting complications and transmission. There is an urgent need to optimise extra-genital STI testing guidelines, for example routine universal testing in high-risk groups.

\section{Chapter 6: Anorectal CT/NG infections missed in women}

Besides in MSM and swingers, anorectal STI are also prevalent in women. It is unknown whether current symptom- and sexual history-based anorectal testing (selective testing) is an effective STI control strategy in women. In this study all women visiting the STI clinic were routinely universally tested at anorectal site. A total of $92 \%(\mathrm{~N}=654)$ of women participated, which indicates high acceptability and feasibility of anorectal testing in women who do not report anal sex. Prevalence of urogenital CT was $11 \%$, prevalence of anorectal CT was $8 \%$. NG infections were not observed. Two thirds of women with anorectal CT did not report anal sex and/or symptoms. Young age was the only determinant associated with anorectal CT. Of all women with anorectal CT, 95\% had concurrent urogenital CT. Of all women with urogenital CT, 71\% also had anorectal CT. Current symptom- and sexual history based anorectal testing in women is not an appropriate control strategy, as two thirds of anorectal CT would be missed. Alternative options could be routine universal anorectal testing, anorectal testing in women with urogenital CT or direct treatment effective for both urogenital and anorectal CT.

\section{Chapter 7: The prevalence and determinants of rectal-only CT/NG}

Anorectal STI can occur as a rectal-only infection or concurrently with a urogenital infection. 
To optimise screening practices, it is important to characterise the target groups in which rectalonly infections occur. Data from the STI clinics in Amsterdam and South Limburg were used to assess factors associated with rectal-only CT/NG infections in MSM (N=9549) and women $(\mathrm{N}=11113)$. The prevalence of anorectal CT $(10 \%)$ and NG $(4 \%)$ was substantial in MSM and prevalence of anorectal CT was also substantial in women (10\%). Anorectal infections occurred mostly as rectal-only infections in MSM and mostly concurrent with other infections in women. Only a few factors were associated with rectal-only infections, and the discriminatory power of associated factors was low in both MSM and women. This makes anorectal screening algorithms based on a priori patient characteristics challenging in practice.

\section{Chapter 8: Anorectal CT load}

Non-Lymphogranuloma venereum anorectal CT is as frequently diagnosed in MSM as in women, but it is unknown whether these infections are comparable in clinical impact and transmission potential. By quantifying bacterial load and identifying determinants associated with high bacterial load in MSM ( $N=90)$ and women $(\mathrm{N}=112)$ we could provide more insight. In this study, we carefully considered transmission potential in terms of bacterial load, as is the case with viral STIs. The mean log anorectal CT load was similar for MSM and women who reported having had anal sex, suggesting similar transmission potential. Anorectal CT load was significantly lower in women who did not report having had anal sex, but the load values were within a similar range. Symptoms were reported equally among the groups and were not associated with anorectal CT load, suggesting similar clinical impact among the groups.

\section{Chapter 9: General discussion}

In chapter 10 we discuss the main findings of this thesis. Anorectal CT/NG infections are contributing to the growing number of observed CT cases in MSM and in women. To prevent sequelae and halt transmission, testing and treatment of anorectal CT/NG are important. Anorectal CT and NG can be successfully detected using NAAT on simple self-taken samples.

In MSM, high prevalence of anorectal CT/NG, low sensitivity of current selective testing, high rates of rectal-only infections, the lack of useful indicators for rectal-only infections and the occurrence of LGV support routine universal anorectal screening. This active approach is already implemented in the UK, but in the US selective symptom- and sexual history based is advised.

In women we found high prevalence of anorectal CT, low sensitivity of current selective testing and high rates of concurrent infections. Several options are discussed to improve anorectal testing in women such as routine universal anorectal screening, anorectal testing in women with urogenital infection, or direct treatment effective for both urogenital and anorectal CT, for example, doxycycline. 
Future studies are needed to fully understand the impact of anorectal infections. For example, the anorectal and urogenital morbidity and transmission potential of anorectal infections, the treatment efficacy for anorectal infections, or the cost-effectiveness of extra-genital testing in women. 

Nederlandse samenvatting 
Dit proefschrift beschrijft verschillende onderzoeken naar de effectiviteit van het huidige internationale en nationale Chlamydia trachomatis (CT) testbeleid, waaronder de huidige testpraktijken en richtlijnen, en geeft aanbevelingen voor toekomstig beleid en onderzoek.

\section{Hoofdstuk 2: CT screening om jongeren te bereiken als aanvulling op de reguliere zorg}

In Nederland werd tussen 2008 en 2010 gestart met Chlamydia Screening via het internet. Het doel van deze screening was het onderbreken van CT transmissie door het opsporen en behandelen van asymptomatische infecties. Dit werd gedaan door jongeren (16-29 jaar) jaarlijks te testen en te behandelen. Een belangrijk onderdeel van een effectieve grootschalige screening is het opsporen van CT positieve jongeren die nog niet bekend zijn bij de reguliere CT zorg zoals de seksueel overdraagbare aandoening (SOA) poli, huisartsen en gynaecologen. We hebben gegevens gebruikt van alle CT zorgverleners in Oostelijk Zuid-Limburg van 2006-2010. Het merendeel $(81 \%, 4298 / 5352)$ van de jongeren die deelnamen aan de CT screening was nog niet eerder getest door de reguliere zorg. Het deel positief geteste jongeren was vergelijkbaar bij jongeren die eerder door reguliere zorg getest waren ( $4.5 \%$ ) en jongeren die niet eerder door reguliere zorg getest waren (4.8\%). Concluderend kunnen we stellen dat CT screening heeft bijgedragen aan de reguliere CT zorg door een groep jongeren te testen die voorheen verborgen waren voor de zorg.

\section{Hoofdstuk 3: CT testen binnen één regio}

De Nederlandse SOA zorg is op een soortgelijke wijze georganiseerd als in Engeland en Australië met een belangrijke rol voor de publieke gezondheidszorg, zoals SOA poli's en huisartsen. SOA poli's richten zich op specifieke risicogroepen, waaronder jongeren ( $<25$ jaar), terwijl huisartsen zich richten op de algemene bevolking. Graag willen we meer inzicht krijgen in test gedrag onder jongeren. We hebben gegevens vergeleken van alle CT zorgverleners in Oostelijk ZuidLimburg; SOA poli's, huisartsen, gynaecologen en CT screening. In totaal zijn er 22.831 CT testen gedaan; $8.2 \%$ ( $n=1868$ ) van de testen was positief voor CT. De SOA poli's hebben meer mannen getest terwijl de huisartsen meer vrouwen getest hebben. Gynaecologen hebben een aanzienlijk deel van de vrouwen getest, maar de meeste CT positieven werden gevonden door SOA poli's en huisartsen. Bij alle zorgverleners werden oudere personen (22 tot 29 jaar) vaker getest, maar waren oudere personen minder vaak positief voor CT. Anale en orale testen worden zelden gedaan buiten de SOA poli. Dit moet onder de aandacht gebracht worden bij de andere zorgverleners. Voornamelijk bij mannen die seks hebben met mannen (MSM) moeten anaal en oraal getest worden vanwege de hoge prevalentie van niet-genitale SOA. Evaluaties zoals deze kunnen helpen om de samenwerking tussen SOA zorgverleners en de zorg te verbeteren, zoals al gedaan wordt in verschillende landen. 


\section{Hoofdstuk 4: Anale CT/NG infecties gemist bij MSM and swingers}

MSM en swingers zijn een belangrijke risicogroep voor SOA, inclusief anale SOA. De huidige richtlijnen adviseren selectief anaal testen op basis van gerapporteerd gedrag; anale seks of anale symptomen. Met deze studie willen we kijken of we met het huidige beleid, selectief testen, infecties missen. Alle MSM en swingers $(\mathrm{N}=1.690)$ die de SOA poli bezochten werden routinematig anaal getest. De prevalentie van anale CT/NG door routinematig anaal testen was $9 \% / 4 \%$ voor homoseksuele MSM, 7\%/3\% voor biseksuele MSM, $4 \% / 1 \%$ voor mannelijke swingers, $1 \% / 0.3 \%$ voor heteroseksuele mannelijke swingers, en $7 \% / 1 \%$ voor vrouwelijke swingers. Met selectief anaal testen wordt tussen de 48-60\% van de anale infecties gemist bij MSM en swingers. In deze hoog risicogroepen kan routinematig anaal testen een betere strategie zijn om transmissie van SOA te onderbreken dan selectief testen.

\section{Hoofdstuk 5: De verdeling van CT/NG over lichaamslocaties bij MSM en hoog-risico vrouwen} Inzicht in de verdeling van SOA over de lichaamslocaties is belangrijk om de controle strategieën voor anale en orale SOA te evalueren. Een mogelijke controle strategie is het gelijktijdig mee behandelen van een anale infectie, als er een genitale infectie is vastgesteld. In deze studie zijn alle MSM ( $N=2436)$ en hoog-risico vrouwen (prostituees en swingers, $N=1321$ ) routinematig getest op anale, orale en genitale CT en/of Neisseria gonorrhoeae (NG). De prevalentie van CT/ NG was $10 \% / 6 \%$ bij MSM en $7 \% / 3 \%$ bij hoog-risico vrouwen. Bij MSM waren tot $76 \%$ van alle infecties geïsoleerd anaal, dus zonder gelijktijdige genitale infectie. Bij vrouwen was dit tot $59 \%$. Het aandeel gecombineerde infecties, dus gelijktijdig een genitale en anale infectie, liep op tot $14 \%$ voor MSM en $54 \%$ voor vrouwen. Uit deze resultaten blijkt dat alleen genitaal testen niet voldoende is, veel anale en orale infecties worden zo gemist. Het gelijktijdig behandelen van een niet-genitale infectie is dus geen goede strategie om complicaties en transmissie te voorkomen. $\mathrm{Er}$ is een grote behoefte aan verbeterde richtlijnen voor niet-genitaal testen, bijvoorbeeld routinematig anaal testen in hoog risico groepen.

\section{Hoofdstuk 6: Anale CT/NG infecties gemist bij vrouwen}

Buiten MSM en swingers, komen anale SOA ook veel voor bij vrouwen. De huidige richtlijnen adviseren selectief anaal testen op basis van gerapporteerd gedrag; anale seks of anale symptomen. Voor deze studie werden alle vrouwen die de SOA poli bezochten routinematig anaal getest om te kijken of we met het huidige beleid, selectief testen, infecties missen. 92\% ( $N=654)$ van de vrouwen wilde meedoen aan de studie. Dit geeft aan dat vrouwen het acceptabel vinden om een anale swab af te nemen, terwijl zij geen anale seks gemeld hebben. Prevalentie van genitale CT was $11 \%$, en de prevalentie van anale CT was $8 \%$. Er waren geen NG infecties gevonden. Twee derde van de vrouwen met een anale CT hadden geen anale seks of anale klachten gemeld. Jonge leeftijd was de enige factor die een verband had met anale CT. Van alle vrouwen met anale CT, had $95 \%$ ook een genitale CT. Van alle vrouwen met een genitale $\mathrm{CT}$, had $71 \%$ ook 
een anale CT. Met het huidig selectieve testbeleid worden dus twee derde van de anale CT infecties bij vrouwen gemist. Mogelijke alternatieven kunnen zijn: routinematig anaal testen van vrouwen, vrouwen met een genitale CT ook anaal testen of een behandeling geven die werkt tegen genitale en anale CT.

\section{Hoofdstuk 7: De prevalentie en factoren die verband hebben met geïsoleerde anale CT/NG}

Anale SOA kunnen geïsoleerd voorkomen, of tegelijkertijd met een genitale infectie. Om het testbeleid te verbeteren is het belangrijk om te weten welke risico groepen geïsoleerde infecties hebben, omdat er niet standaard anaal getest wordt. We hebben data gebruikt van de GGD Amsterdam en de GGD Zuid Limburg om te kijken welke factoren een verband hebben met geïsoleerde anale $\mathrm{CT} / \mathrm{NG}$ infecties bij MSM ( $N=9549)$ en vrouwen ( $\mathrm{N}=11.113)$. De prevalentie van anale CT (10\%) en NG (4\%) was substantieel bij MSM en de prevalentie van anale CT was ook substantieel bij vrouwen (10\%). Bij MSM waren de meeste anale infecties geïsoleerd, terwijl bij vrouwen de meeste anale infecties gecombineerd waren met een genitale infectie. Slechts een aantal factoren hadden een verband met geïsoleerde anale infecties, en dit was niet voldoende om een onderscheid te maken tussen hoog- en laag risico personen. Dit maakt het lastig een testbeleid te ontwikkelen op basis van persoonskenmerken of indicatoren.

\section{Hoofdstuk 8: Anale CT load}

Anale CT, geen Lymphogranuloma venereum, komt even vaak voor bij MSM als bij vrouwen. Het is echter onbekend of deze infecties vergelijkbaar zijn tussen deze groepen wat betreft klinische impact (klachten) en transmissie potentie. Met deze studie proberen we hier meer inzicht in te krijgen door de bacteriële load (het aantal bacteriën per swab) te bepalen bij MSM (N=90) en vrouwen ( $N=112$ ), en te kijken welke factoren van invloed zijn op de load. In deze studie bekijken we transmissie potentieel met de load, zoals bij virale SOA. Een hoge load betekent dan een hoog transmissie potentieel. De gemiddelde anale CT load was hetzelfde bij MSM en vrouwen die anale seks gemeld hadden, dit suggereert een gelijk transmissie potentieel. De anale CT load was lager in vrouwen die geen anale seks gemeld hebben, maar de waardes waren in dezelfde marge als personen die wel anale seks gemeld hebben. Klachten waren gelijk bij MSM en vrouwen, en ze hadden geen invloed op load. Dit suggereert een gelijke klinische impact bij MSM en vrouwen.

\section{Hoofdstuk 9: Algemene Discussie}

In hoofdstuk 10 bespreken we de belangrijkste bevindingen van dit proefschrift. Anale CT/NG dragen bij aan het stijgende aantal CT infecties bij MSM en vrouwen. Om complicaties te voorkomen en transmissie te stoppen is het belangrijk om te testen voor anale infecties en deze ook te behandelen. Anale CT en NG kunnen getest worden met een NAAT met behulp van makkelijke zelf afneembare swabs. 
In MSM pleiten de hoge prevalentie van anale CT/NG, de lage sensitiviteit van het huidige selectieve testen, hoge aantallen geïsoleerde anale infecties, een gebrek aan bruikbare indicatoren om geïsoleerde anale infecties op te sporen en het voorkomen van ernstige LGV infecties voor routinematig anaal testen. Deze actieve aanpak wordt al gebruikt in het Verenigd Koninkrijk, maar in de VS wordt nog selectief getest op basis van anale seks en symptomen.

Bij vrouwen vonden we een hoge prevalentie van anale $\mathrm{CT}$, lage sensitiviteit van het huidige selectieve testen en hoge aantallen gecombineerde anale en genitale infecties. Verschillende opties worden besproken om het anaal testen van vrouwen te verbeteren zoals routinematig anaal testen, anaal testen van vrouwen met genitale infectie, of een behandeling geven die werkt tegen genitale en anale $\mathrm{CT}$.

Toekomstige studies zijn nodig om de impact van anale infecties volledig te begrijpen. Bijvoorbeeld het bekijken van anale en genitale morbiditeit, transmissie potentieel van anale infecties, de effectiviteit van de behandeling van anale infecties, en de kosteneffectiviteit van anaal en oraal testen bij vrouwen. 

Dankwoord 
I did it! Maar natuurlijk niet alleen. In dit dankwoord wil ik iedereen bedanken die een bijdrage aan dit proefschrift heeft geleverd, op allerlei denkbare manieren. Wat zijn ze voorbij gevlogen, die 4 jaar als promovendus.

Als eerste wil ik de afdeling Seksuele Gezondheid van de GGD Zuid Limburg bedanken. De doktersassistenten voor het verzorgen van de logistiek, de artsen voor de vele vragen en het meedenken, en natuurlijk de verpleegkundigen voor alle bovengenoemde taken, het werven, communiceren en wat al niet meer. Door jullie enthousiasme en inzet hebben er zoveel mensen aan de studies meegedaan. Het vergt kennis en professionaliteit om personen een anale swab te laten afnemen die geen anale seks hebben gehad. Het was niet altijd makkelijk, het extra werk van de onderzoeken kwam bovenop de reguliere werkzaamheden. Het is jullie gelukt. Dank aan de unithoofden Anne-Marie Niekamp en Mieke Steenbakkers, dat dit mogelijk is. Uiteraard wil ik ook de deelnemers aan de studies bedanken.

Prof dr. Christian Hoebe, beste Christian, bedankt dat je mij de kans hebt gegeven om op jouw afdeling te komen werken, en later zelfs te promoveren. Deze kans kwam voor mij op het perfecte moment. Jouw enthousiasme werkt aanstekelijk, en door jouw positieve houding was geen probleem onoverkomelijk. Jij leerde me hoofd- en bijzaken te onderscheiden, knopen doorhakken en natuurlijk de truc met het bierviltje! Naast je professionele kwaliteiten beschik je over een dosis humor die de vele overleggen, congressen en treinreizen significant leuker gemaakt heeft.

Dr. Nicole Dukers-Muijrers, beste Nicole, bedankt dat je me wegwijs hebt gemaakt in de wereld van de epidemiologie en het schrijven van artikelen. Wat heb ik veel van je geleerd. Door jouw kennis en expertise mag ik me nu zelfs epidemioloog noemen. Talloze SPSS outputs hebben de revue gepasseerd, nog niet te spreken over $X$ versies van manuscripten. Jij maakte altijd tijd om ze kritisch door te nemen. Je stond altijd voor me klaar met feedback, tips en adviezen, maar je liet me ook zelf keuzes maken en verantwoordelijkheid nemen.

Ik mocht als groentje zelfs naar Québec Canada om mijn eerste ingediende abstract te presenteren op een internationaal congres! Dit was nog voordat ik was begonnen aan mijn promotie traject. Door jullie vertrouwen in mij, heb ik vertrouwen in mijzelf gekregen.

Collega's van de GGD, afdeling Seksuele Gezondheid, Infectieziekten en Milieu, bedankt voor jullie interesse in zowel mijn proefschrift als mijn persoon. Dames van het secretariaat, bedankt voor de ondersteuning. Een speciaal word van dank aan Ine en Helen. Ine, al dat opschoonwerk... dit zegt voldoende denk ik! Bovendien was je altijd geïnteresseerd, en heb je met me meegeleefd bij alle pieken en dalen. En Helen, wat moeten we zonder Helen? Werkelijk alles kon ik je vragen, en binnen no time had je het geregeld. 
De onderzoekers, wat begon als kleine club is uitgegroeid tot een heuse unit, inclusief blitse groepsfoto. Laura, ik begon bij de GGD met jou als kamergenoot en ik kon je altijd vragen om hulp. Inmiddels hebben we al aardig wat data vraagstukken opgelost, ik hoop dat we in de toekomst kunnen blijven samenwerken. Angelique, bedankt voor alle leuke momenten, waaronder ons tripje naar Edinburgh. I make proefschrift! Steffi en Jeanne, de volgende lichting bij de GGD. Ik geniet elke dag van onze lunchwandelingen, en natuurlijk het 'buurten' bij jullie. Heel veel succes met jullie promotietrajecten! Lisanne, mijn nieuwe kamergenoot. Je trof me niet in de meest rustgevende fase van mijn promotie, maar desondanks liggen we regelmatig in een deuk. Ik hoop dat jij binnenkort ook een promotie traject kunt starten, mijn hulp staat altijd klaar. Last but not least, Kevin, mijn paranimf. Dit was geen moeilijke keuze. Samen zijn we aan dit avontuur begonnen en ik kon altijd bij je terecht, met een lach of met een traan. Ik heb veel van je geleerd, meer dan jij je zelf realiseert. En wat hebben we gelachen al die jaren, humor relativeert, dat weten wij als geen ander. Ik vind het erg jammer dat we geen collega's meer zijn, maar natuurlijk hoop ik dat we elkaar nog vaak zien. Zet hem op met de laatste loodjes van je proefschrift!

Ik wil ook mijn collega's bij de Medische Microbiologie bedanken. Petra, onze samenwerking heeft veel toegevoegde waarde. Bedankt dat ik jullie data en expertise mocht gebruiken, onder andere voor het load artikel, er ging een wereld voor me open. Anne D, bedankt voor al die load bepalingen. Het was een hele klus, maar de resultaten mogen er zijn. Succes met je opleiding en natuurlijk je promotie.

Graag wil ik ook alle coauteurs bedanken voor hun medewerking. In het bijzonder de projectgroep van de Chlamydia Screening Implementatie (CSI) collega's van de GGD Amsterdam, GGD Rotterdam en het RIVM, bedankt voor de prettige samenwerking, ik hoop dat we dit in de toekomst kunnen voortzetten!

Leden van de promotiecommissie, bedankt dat u wilde plaatsnemen.

Lieve vrienden, jullie zorgden voor de ontspanning! Allereerst de chicks en David (hihi), bedankt voor de leuke weekendjes, festivals, verjaardagen, feestjes en bioscoopbezoekjes.

Een aantal mensen wil ik graag persoonlijk bedanken. Melissa, Valérie en Astrid S, wat heerlijk die Zumba lessen! Op die momenten kon ik alles letterlijk van me af schudden. Jos\&Melissa, we delen onze liefde voor het Bourgondische leven. Bedankt voor de gezellige etentjes en de goede gesprekken. Jos, fijn dat je me hebt laten kennismaken met de hobby hoddelen, even de gedachten ergens anders op zetten werkt verhelderend. Joëlle, hoelang zijn we al bevriend? Binnenkort ons 25 jarig jubileum vieren...Bedankt dat je er bent op de momenten dat het nodig is. Denise, bedankt voor je luisterend oor, en natuurlijk het 'babysitten' op Bèr. Carola, we kunnen 
het nog steeds! Anne M, als het mocht was jij mijn derde paranimf. Dat was wel een moeilijke beslissing. De tijd samen in Amsterdam en Brussel, en de vele jaren tot nu toe, zal ik nooit vergeten. Jij stond altijd paraat met de goede woorden. Ik hoop dat we nog lang bevriend zullen blijven en daar twijfel ik niet aan :--. Stefanie, chama, mijn tweede paranimf. Hoe kan ik het beter zeggen: contigo tenía el mejor tiempo de mi vida. We hebben samen een dosis levenservaring opgedaan in de vorm van 6 maanden bivakkeren in Venezuela, en nu gaan we allebei promoveren. Pana, ik ben blij dat jij achter mij staat tijdens mijn promotie. Wie weet wat nog op ons pad komt in de toekomst, nos vemos!

Maria, Hub, Frederique en Regine, bedankt voor het warme welkom bij de familie Gustings. Jullie waren altijd geïnteresseerd in mij en de promotie, zie hier het resultaat!

Hans, Leon en Sander, jullie behoren ook tot mijn familie. Hans bedankt voor alles.

Petetante Diana, bedankt voor je vertrouwen en je trots. Je bent een doorzetter, dat heb ik van jou geleerd. Ik weet zeker dan bon-papa en Noé vandaag bij ons zijn en meegenieten van deze dag.

Bonne-maman, lieve Francisca, door $\mathrm{u}$ ben ik geworden wie ik nu ben. $\mathrm{U}$ heeft mij geleerd het beste uit mezelf te halen en altijd te blijven leren. Bedankt voor alles.

Mutti, bedankt dat je er altijd voor mij was, ook als ik meer te klagen dan te kletsen had. Door jou heb ik geleerd wat hard werken is. Je hebt me altijd gestimuleerd mijn eigen weg te gaan, ook al was dit niet het gebaande pad. Leef mam, bedankt voor de liefdevolle opvoeding. Ik had geen betere moeder kunnen wensen.

Vincent, miene leeve. Jij stond aan de wieg van mijn beslissing om te gaan promoveren, nog voordat we samen door het leven gingen. Ik ben zo gelukkig met jou, woorden schieten te kort. Bedankt dat je altijd voor me klaar staat. Ich hou van dich <3. 

Curriculum Vitae 
Geneviève van Liere werd geboren op 15 april 1987 in Heerlen. Zij behaalde haar VWO diploma in 2005 aan het Stella Maris college te Meerssen. In 2005 is ze verhuisd naar Amsterdam en begonnen aan de studie Gezondheidswetenschappen aan de Vrije Universiteit (VU). Voor haar bachelor stage aan de Vrije Universiteit Brussel onderzocht ze de kwaliteit van leven bij terminale longkanker patiënten. In 2008 startte ze met de Master Infectieziekten aan de VU Amsterdam. Haar afstudeerstage heeft ze in Venezuela gedaan, waar ze de prevalentie, risicofactoren en mogelijke besmettingsbronnen onderzocht van de darmparasiet Schistosoma mansoni. Haar Master diploma behaalde ze in mei 2010, en in oktober datzelfde jaar is ze begonnen als onderzoeker/datamanager bij de GGD Zuid Limburg. Als onderzoeker heeft ze aan diverse onderzoeken meegewerkt o.a. over $Q$ koorts en SOA. Gedurende haar loopbaan bij de GGD verzorgt ze het datamanagement van de SOA data, en heeft ze haar registratie Epidemioloog A en B behaald. In 2011 startte ze met haar promotie onderzoek, in samenwerking met de afdeling Medische Microbiologie van het Maastricht Universitair Medisch Centrum (MUMC+) en de School for Public Health and Primary Care (CAPHRI), onder leiding van Prof. dr. Christian Hoebe en dr. Nicole Dukers-Muijrers. 

Portfolio 


\section{Courses and training}

2015

Epidemiologist B (post doctoral epidemiologist) 2011-2015, the Dutch Society of Epidemiology.

2013

Epidemiologist A (pre-doctoral epidemiologist), the Dutch Society of Epidemiology.

Advanced Statistical Techniques by Maastricht University.

Creativity Workshop by the Centre for the Development of Creative Thinking (COCD).

2012

Multilevel Analyses of Longitudinal Data by Maastricht University.

Project Management by Teams@Work.

Mind Mapping, Memory Training and Speed Reading by World of Minds.

2011

Heuvelland Course: Effective Writing and Publishing Scientific Papers.

Best Practices in Publishing Clinical and Public Health Research by Eduardo L Franco.

\section{Presentations}

\section{International}

19th Biennial meeting of the International Society for Sexually Transmitted Diseases Research Congress 2011, Quebec city, Canada.

\section{National}

- 10th Annual Amsterdam Chlamydia Meeting 2015, Amsterdam.

- National HIV/AIDS Conference 2014, Amsterdam.

- Expert Meeting 2014, RIVM Bilthoven.

- Research day Medical Microbiology 2014, Maastricht.

- Research day Medical Microbiology 2013, Maastricht.

- 8th Annual Amsterdam Chlamydia Meeting 2012, Amsterdam.

- Expert Meeting 2012, RIVM Bilthoven.

- Annual CAPHRI Research Meeting 2011, Maastricht. 



\section{List of publications}


van Liere GAFS, Dirks JAMC, Hoebe CJPA, Wolffs PF, Dukers-Muijrers NHTM. Anorectal Chlamydia trachomatis Load Is Similar in Men Who Have Sex with Men and Women Reporting Anal Sex. PLoS ONE 2015 10(8): e0134991. doi:10.1371/journal.pone.0134991.

Heijer den CDJ, Liere van GAFS, Hoebe CJPA, Bergen van JEAM, Cals JWL, Stals FS, Dukers-Muijrers NHTM. Who tests whom? A comprehensive overview of Chlamydia trachomatis test practices among different STI care providers for urogenital, anorectal and oropharyngeal sites in young people. Sex Transm Infect 2015 Published Online First: [12-08-2015] doi:10.1136/ sextrans-2015-052065.

Dukers-Muijrers NHTM, Liere van GAFS, Wolffs PF, Heijer den CDJ, Werner MI, Hoebe CJPA. Antibiotic use before chlamydia and gonorrhea genital and extragenital screening in the sexually transmitted infection clinical setting. Antimicrob Agents Chemother 2015;59:121-8.

van Liere van GAFS, van Rooijen MS, Hoebe CJPA, Heijman T, de Vries HJC, DukersMuijrers NHTM. Prevalence of and factors associated with rectal-only chlamydia and gonorrhoea in women and men who have sex with men. PLoS ONE 10(10): e0140297. doi: 10.1371/journal.pone.0140297.
Liere van GAFS, Dukers-Muijrers NHTM, Bergen JEAM van, Götz HM, Stals F, Hoebe CJPA. The added value of chlamydia screening between 2008-2010 in reaching young people in addition to chlamydia testing in regular care; an observational study. BMC Infectious Diseases 2014; 14:612.

Liere van GAFS, Hoebe CJPA, Wolffs PFG, Dukers-Muijrers NHTM. High co-occurrence of anorectal chlamydia with urogenital chlamydia in women visiting an STI clinic revealed by routine universal testing in an observational study; a recommendation towards a better anorectal chlamydia control in women. BMC Infectious Diseases 2014; 14:274.

Liere van GAFS, Hoebe CJPA, DukersMuijrers NHTM. Evaluation of the anatomical site distribution of chlamydia and gonorrhoea in men who have sex with men and in high-risk women by routine testing: cross-sectional study revealing missed opportunities for treatment strategies. Sex Transm Infect 2014;90:58-60.

Hofstede SN, Tami A, van Liere GA, Ballén D, Incani RN. Long-term effect of mass chemotherapy, transmission and risk factors for Schistosoma mansoni infection in very low endemic communities of Venezuela. Acta Trop. 2014 Dec;140:68-76. 
Liere van GAFS, Hoebe CJPA, Niekamp AM, Koedijk FDH, Dukers-Muijrers NHTM.

Standard symptom- and sexual history-based testing misses anorectal Chlamydia trachomatis and Neisseria gonorrhoeae infections in swingers and men who have sex with men. Sex Transmitted Dis 2013; 40(4):285-289.

Dukers-Muijrers NHTM, van Liere GAFS, Hoebe CJPA. Re-screening Chlamydia trachomatis positive subjects: a comparison of practices between an STI clinic, general practitioners and gynaecologists. Sex Transm Infect. 2013 Feb;89(1):25-7.

Liere van GAFS, Dukers-Muijrers NHTM, Hoebe CJPA. Chlamydia en gonorroe alleen urogenital testen onvoldoende. Ned.

Tijdschrift Geneeskunde. 2013;157: C1948.
Koedijk FDH, van Liere GAFS, Peters RPH, Hoebe CJPA, van der Sande MAB, Götz HM, van Bergen JEAM. Meerderheid anale chlamydia en gonorroe-infecties gemist zonder routinematig testen. SEKSOA 2012:16. 

Valorisation of the thesis 


\section{Relevance for society and economy}

The research project presented in this thesis has a clear social and economic relevance, in addition to scientific relevance.

Sexually transmitted infections (STI) clinics provide free and anonymous testing to various risk groups such as youngsters <25 years of age, commercial sex workers (CSWs), swingers, men who have sex with men (MSM), people suffering from symptoms and people with $>3$ sex partners. Targeted care is delivered by trained nurses who take medical and sexual history and provide testing for sexually transmitted infection (STI) s such as Chlamydia trachomatis (CT) and Neisseria gonorrhoeae (NG). Long term complications of STIs can be ectopic pregnancy, infertility, pelvic inflammatory disease and urethritis. Municipalities and the government finance this specialized STI care (nurses, medical doctors); however research is externally funded through various sources such as scholarships.

Testing guidelines are provided by the National Institute for Public Health and the Environment (RIVM). These guidelines are established by a national group of experts consisting of medical doctors, epidemiologists, and policy makers etc. who evaluate literature and use their own expertise. This thesis provides an evaluation of the effectiveness of the current procedures to control CT. Current guidelines comprise standard urogenital testing in every consultation. Anorectal testing is performed after report of anorectal symptoms and/or anal sex, that is, selective testing on indication. In the studies in this thesis, routine universal anorectal screening was used instead of selective testing on indication in MSM, swingers and women. We found that half of anorectal infections would have been missed using selective testing, in women this was even two thirds. This means that of all women with an anorectal STI, only one third reported anal sex or symptoms. Therefore, current guidelines lead to insufficient case management of MSM, swingers and women with an anorectal STI infection. The societal impact of this insufficient case management is substantial, as anorectal STI prevalence is $10 \%$ among STI clinic visitors. STIS have a public health and clinical impact, in terms of symptoms and transmission. The clinical impact of non-LGV anorectal STIs is limited as only $5 \%$ reported symptoms. The majority of MSM have a rectal-only infection, while the majority of women have a concurrent anorectal urogenital infection. In this thesis theories are presented to explain this difference, for example autoinoculation with vaginal secretions in women, especially since the majority of women with an anorectal infection do not report anal sex. These anorectal infections can facilitate transmission between individuals by anal sex, as is suggested in MSM. Possibly, these anorectal infections in women could also lead to reinfection within an individual, causing reproductive health problems. Moreover, anorectal STI facilitate HIV transmission, which is primarily a problem in MSM. Altogether, the clinical impact of anorectal infections should not be underestimated. HIV infection and reproductive health problems contribute to health care costs in the Netherlands. Yearly, 55.500 CT diagnoses are made; 32.000 in women and 23.500 in men. The risk of inferti- 
lity by a chlamydia infection in women is estimated differently by several studies. Mathematical studies estimated a probability of $10 \%$, with a range of 2-35\%. However, a review reported a lower risk of 0.1 to $4.6 \%$. The cost effectiveness of routine universal anorectal screening, as described in this thesis, is estimated at $\$ 1.400$ by avoiding serious complications.

\section{Target groups}

The results and conclusions presented in this thesis are of importance for the whole sexual active population who have unprotected sexual contact. Especially MSM and young women should be aware of the common occurrence of anorectal infections. For MSM, mainly because of high transmission potential by frequent changing of partners. For women, because of the potential complications due to untreated anorectal infections, even when anal sex is not reported. The study population in this thesis was STI clinic attendees, which implicates a high risk group for STI. However, STI are also frequently diagnosed at the general practitioner (GP), as described in this thesis. In our local STI clinic, policy has already changed as an implication of the research in this thesis; MSM and swingers are routinely universally tested for anorectal STI. Before the occurrence and importance of anorectal infections can be highlighted, the focus should be on STI testing in general, as this is the first step in the process. Collaborating and sharing knowledge with other STI care providers, such as gynaecologists and GPs, would improve STI care in general. Policy makers are also a target group regarding their role in designing and implementing testing guidelines which are used nationwide by STI care providers. At last, local policy makers at the municipality play a role as STI research and policy evaluation is not financed by the municipality. This makes research and policy evaluation subjective to external funding, which is not a continuous flow of funds.

\section{From activities and innovation to planning and realisation}

\section{Research (1)}

Research is needed for actual change of standard operating procedures, especially on an international level. Research leads to answers, but even more to questions. Recently, funding has been granted to carry out an innovative spin off study, which will provide answers to the remaining questions. The aim of the study is obtain insight into the acquisition and transmission of CT between and within persons. Urogenital and/or anorectal CT positive women will be followed over time after CT treatment. Women included in the study will take 24 consecutive swabs in a timeframe of 12 weeks, 12 vaginal swabs and 12 anorectal swabs. Multiple questionnaires will provide information on sexual risk behaviour throughout the study. 


\section{Research (2)}

Communicating research results is a key item for valorisation. When an article is published, a brief summary in Dutch is send by email to a multidisciplinary group of people working in the same field, for example, GPs, researchers and policy makers. Moreover, results are communicated by our academic website, including a short interview with the researcher. These outreach methods could be improved by launching a website about our research group. This website should include a short background of all researchers, published papers and ongoing research projects. A request for this was made by the communications department.

\section{Policy}

It is important that policy makers are aware of the findings in this thesis for (1) re-evaluate literature and consider improving testing guidelines and (2) providing funding for future research. Moreover, once a year a national expert meeting is held at the National Institute of Public Health and the Environment. Policy makers, medical doctors and researchers are present at this meeting to present research and discuss findings with each other. Moreover, policy makers visited our setting to talk about practice and research. This leads to connections for future research and thereby to a better chance for funding.

In my opinion, research should become a part of standard care in the STI clinic in contrast to current situation in which additional funding has to be acquired to carry out research/policy evaluation. Ideally, research is financed by the municipalities in addition to standard care. This would make STI research, which in fact often is an evaluation of care, accessible to both (1) those we want to help (clients) and (2) those funding (municipalities). If there would be a budget accessible for research within care, results could be easily passed to clients by various ways such as the public health service website, outreach to schools and local folders spread by the public health service or municipality. At the moment financial resources are too small to carry out these kinds of actions to promote the research among (possible) clients and policy makers. When research is part of standard care, the researchers are obliged to give feedback to the municipality about the research. This works two ways, results are presented to local policy makers and local policy makers can see the importance of research for practice and stimulate it by financial resources. At the moment a folder with testing rates and positivity rates among various risk groups is already given to local policy makers, research could be added to this document. However, I think it is important to start with presenting results to local policy makers which will lead to personal interaction and mutual interest.

\section{Influence public debate/media}

Since the subject of this thesis comes with taboos and stigma, it is necessary to carry out results and make the subject STI testing a topic for discussion. This will have impact on policy makers; STIs are a problem, and will always be if investments are not made, as well as our target group of people we want to reach for testing but have barriers to do so. Publications have already 
attracted media attention and local television regularly interviews medical doctors and nurses about actual topics in standard care. However, this could also be done for research and even on a national level, potentially in collaboration with other researchers in the field. We have never actively approached media to carry out our research because often more research is needed before we can make statements about it. Therefore, it is not an appropriate source to carry out results of research in early stages since this could lead to misinterpretation. However, the media often approaches our organisation first when an article is published. This indicates that our research is of interest to journalists and the community. In this way, attention is given to STIs in the public media which reaches a large group of individuals. To carry out this thesis in a broad way, an official press release will be issued when this thesis is printed. In addition to the printed thesis, a summary leaflet in A4 will be created by a professional copywighter to summarize the key points in a concise way. This leaflet is written in non-academic Dutch language, which makes it readable for a wider community.

\section{Spin off (1)}

Training and education can draw attention to STI among youngsters, which is a well known target group. Since end of 2012, schools are required to pay attention to sexuality and sexual diversity by the government. Schools also have the task of ensuring a safe social climate in and around the school and to promote good citizenship. A website and curriculum named 'Sekswijzer' have been developed with general information about STIs, sexual diversity, sexual violence and defensability. Ideally, a regional campaign could be carried out to promote STI testing among youngsters. This group would benefit the most as they are a high risk group for STI, including anorectal STI. To promote research findings and make this information appealing to youngsters, social media could be helpful. We plan to open a twitter account to communicate research results using a humorous style, which will appeal to youngsters.

\section{Spin off (2)}

Besides the STI clinics, GP's also provide STI testing; they serve about one third of the population. Informing GPs and involve them in future research projects would have the largest impact on STI control. At the moment, several innovative research projects are set up which involve collaboration with GPs, such as partner warning after a diagnosed STI and projects on hepatitis. This could be extended to CT, especially anorectal CT, as those data are limited for GPs. Publishing articles in national journals such as 'Nederlands tijdschrift voor geneeskunde' and 'Huisarts en Wetenschap' can have an impact, but not all GP's read it and even smaller part would change their practice. It would be best to start with informing by email, since time is scarce for GPs, or visit some practices to present our results. Eventually presenting results to the expert group which formulate the GP guidelines would have the largest impact. Together with GPs, gynaecologists should be informed and involved in a similar way, since they perform a substantial share of CT tests in this region. In conclusion, collaboration with STI care providers in any way is beneficial for STI control. 



\section{"A person who never made a mistake never tried anything new.}

Albert Einstein 


